\title{
MEAN VALUE MODELING AND CONTROL OF A DIESEL ENGINE USING NEURAL NETWORKS
}

\author{
Yasser Yacoub
}

Dissertation submitted to the College of Engineering and Mineral Resources

at West Virginia University in partial fulfillment of the requirements

for the degree of

\author{
Doctor of Philosophy \\ in \\ Mechanical Engineering
}

Christopher M. Atkinson, Sc.D., Chair

Nigel Clark, Ph.D.

Marcello Napolitano, Ph.D.

Ian Christie, Ph.D.

Theresa Long, Ph.D.

Department of Mechanical and Aerospace Engineering

\author{
Morgantown, West Virginia \\ 1999
}

Keywords: Mean Value Modeling, Control, Diesel Engine, Neural Networks 


\section{ABSTRACT \\ MEAN VALUE MODELING AND CONTROL OF A DIESEL ENGINE USING NEURAL NETWORKS}

\section{Yasser Yacoub}

Increasingly stringent emissions legislation and demands for improved fuel economy have mandated the need for advanced control algorithms and complicated the diesel engine calibration procedure. To this end, a neural networkbased mean value model of a modern turbocharged direct injection diesel engine has been developed and validated. For a pre-specified engine speed schedule and control vector trajectory, the engine model was shown to produce accurate predictions of the turbocharger manifold charging dynamics and combustion efficiency through engine brake torque predictions. The mean value model was coupled with several sub-models to predict exhaust gaseous and particulate emissions and satisfactory predictions were reported over highly transient engine test schedules.

The mean value model was used to develop and validate through simulation a neural network-based engine torque controller for both non-governed as well as governed engine operation. Two types of proportional governors were considered where one governor employed a more aggressive fueling strategy than the other. The engine performance and exhaust gas emissions for both strategies were quantified through simulation, showing steeper rises in torque and larger excursions in transient emissions for the more aggressive fueling strategy. The controller was adapted online using the standard back-propagation algorithm. For a pre-specified engine speed schedule and desired engine torque trajectory, excellent torque tracking was predicted using the neural network (NN) controller over transient operation compared to a classical proportional plus integral (PI) controller, which was tuned heuristically.

The mean value model was also used to develop and validate through simulation a neural network-based all-speed governor. For a pre-specified engine load schedule and accelerator position trajectory, accurate tracking was predicted for the desired engine speed for both classical and NN-based controllers under high load transients.

For the test engine used, it was shown through simulation that tighter control of engine torque over the Federal Test Procedure (FTP) cycle resulted in higher brake specific emissions of carbon dioxide, oxides of nitrogen, and particulate matter. Also, the EPA validation criteria for the prescribed engine torque over the FTP cycle allow for significant variations in brake specific emissions, especially particulate matter, total hydrocarbons, and carbon monoxide emissions, while still meeting the legal requirements for a valid engine certification test. 


\section{ACKNOWLEDGEMENTS}

The author wishes to express his gratitude to his thesis advisor, Dr. Chris Atkinson, for the invaluable opportunity he has given him to work on this very interesting subject. Thanks for many unlimited office hours of sincere discussions on engine controls, a subject greatly complicated by confidentiality agreements and propriety information.

The author wishes to thank his committee members, specifically Dr. Napolitano for accepting him as a 'guest' in his advanced control class and Dr Long for many ideas on the virtual sensor approach. Thanks are also due to Dr Clark for his trust and for the very enlightening hint that 'Diesel engines are speed and not load controlled', and Dr. Christie for outstanding three classes in numerical analysis.

The author wishes to acknowledge the effort expended by his colleagues at WVU Engine Research Center in instrumenting the engine and conducting part of the reported testing. Thanks are also due to Dr. Greg Thompson for providing the random cycle generator program.

On a private note I would like to acknowledge my family for being so wonderful.

Morgantown, WV

March 1999

Y. M. Yacoub 


\section{TABLE OF CONTENTS}

ABSTRACT

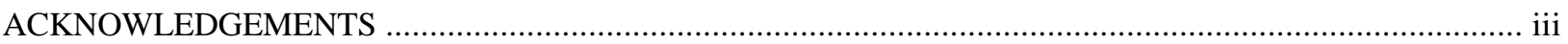

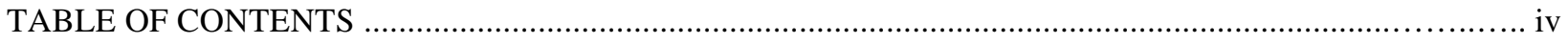

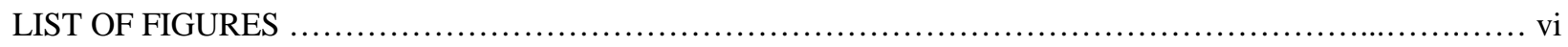

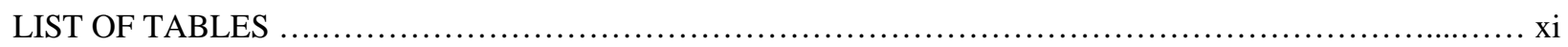

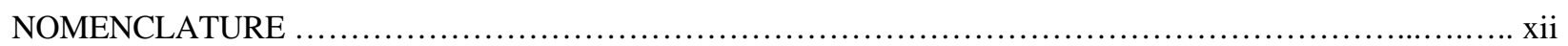

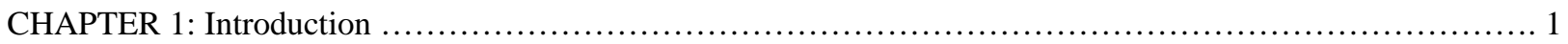

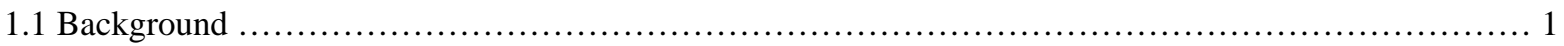

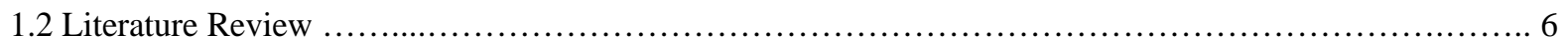

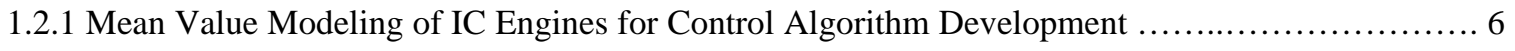

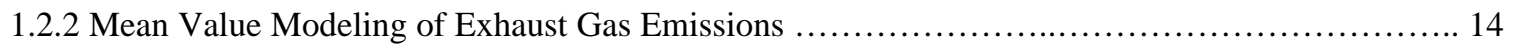

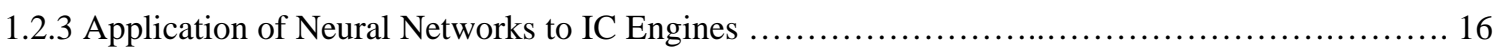

1.2.4 Control of HD CI Engines on Transient Dynamometers......................................... 25

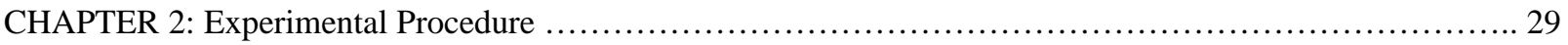

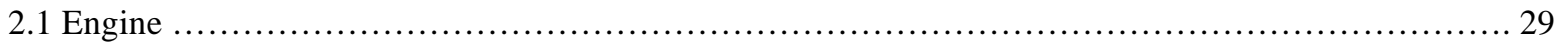

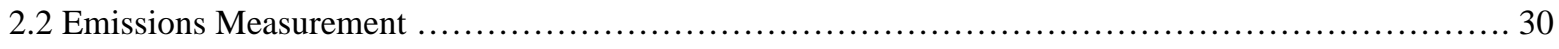

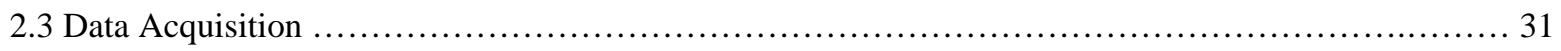

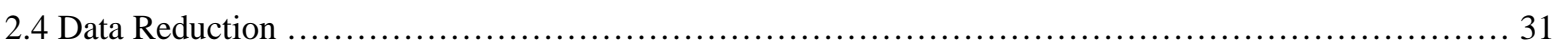

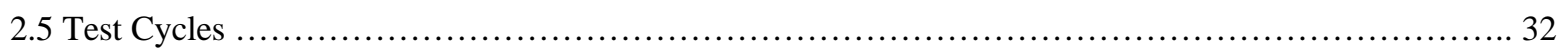

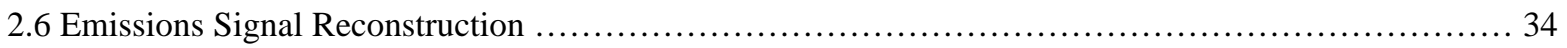

CHAPTER 3: Neural Network-based Mean Value Engine Modeling ................................... 46

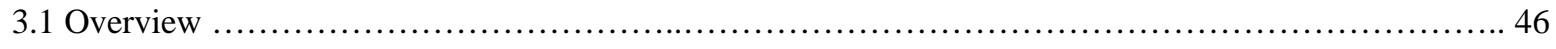

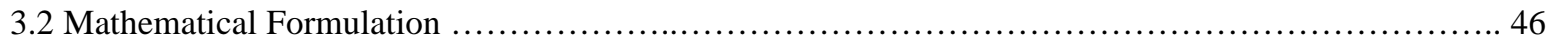

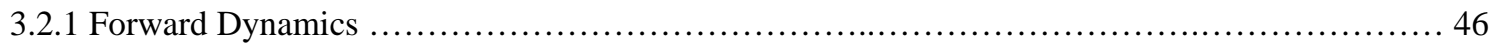

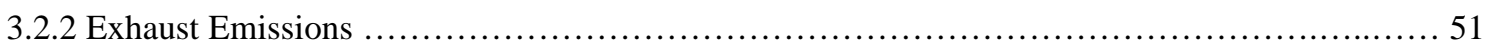




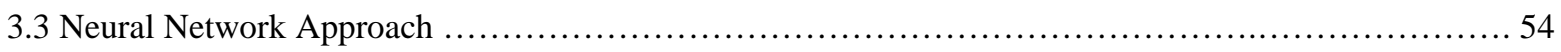

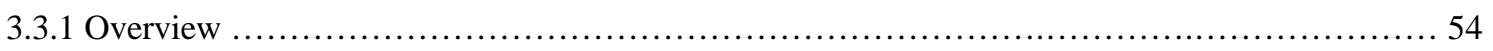

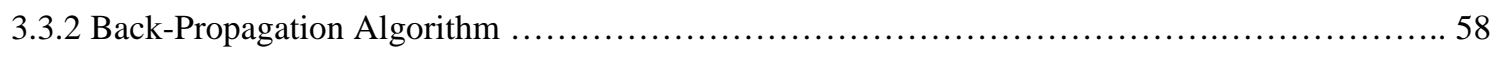

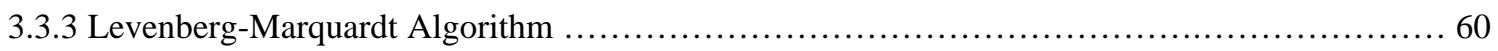

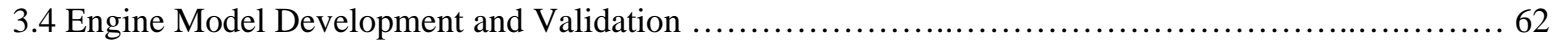

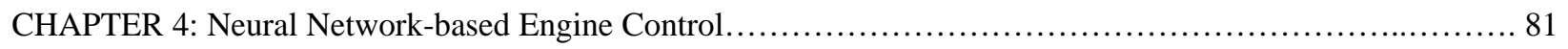

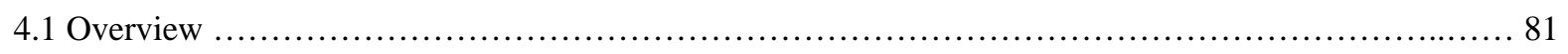

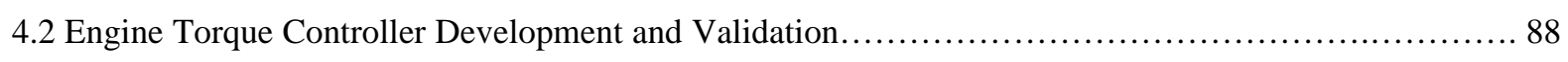

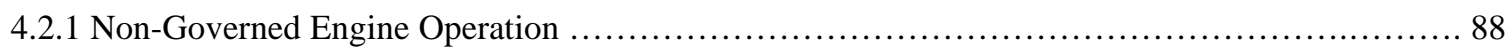

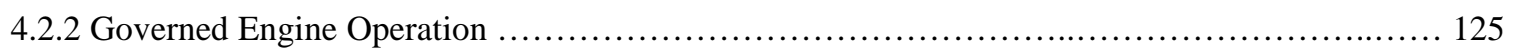

4.3 Engine Speed Controller Development and Validation ......................................... 139

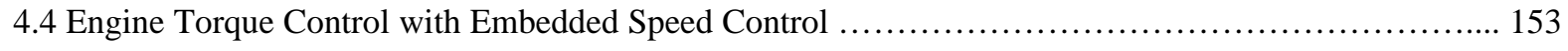

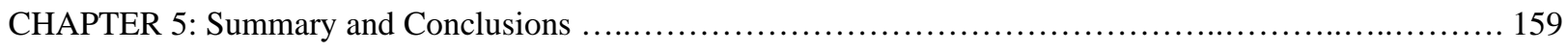

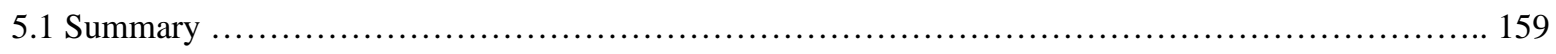

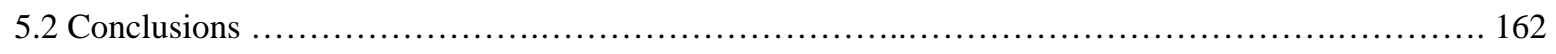

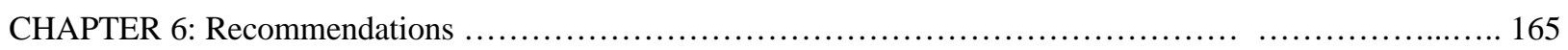

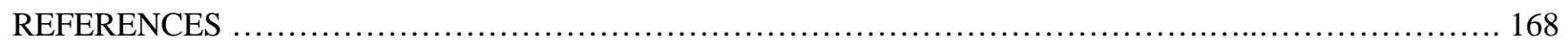

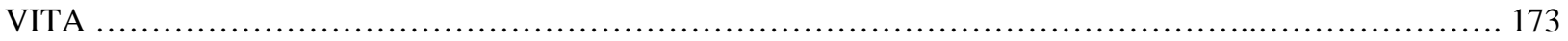




\section{LIST OF FIGURES}

Figure 1.1 Control algorithm synthesis in a rapid prototyping environment f............................... 2

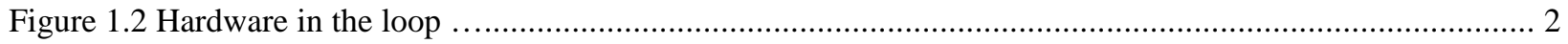

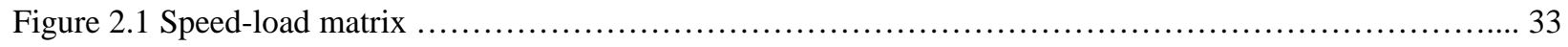

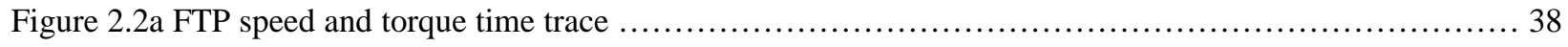

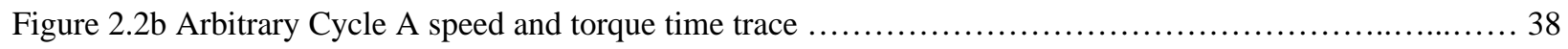

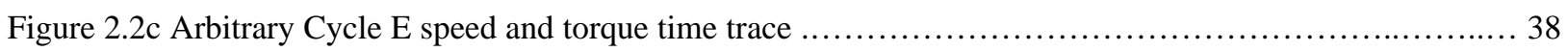

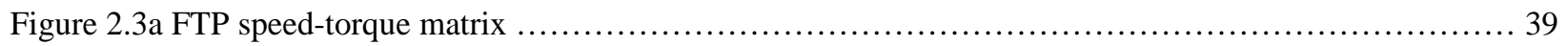

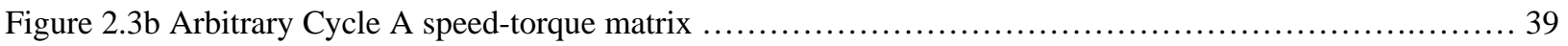

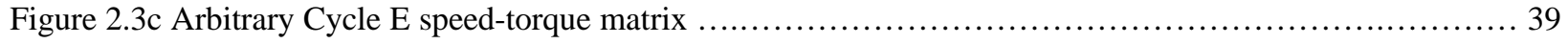

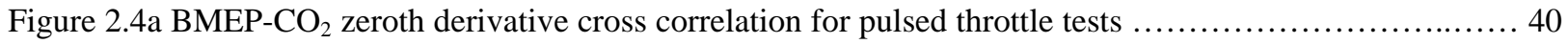

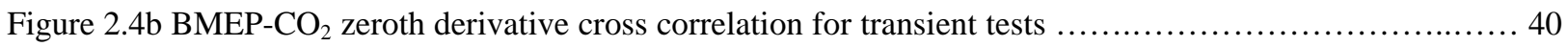

Figure 2.4c BMEP-CO $\mathrm{CO}_{2}$ first derivative cross correlation for pulsed throttle tests $\ldots \ldots \ldots \ldots \ldots \ldots \ldots \ldots \ldots \ldots \ldots . \ldots \ldots$

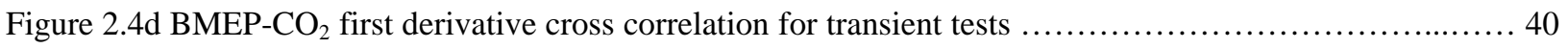

Figure 2.5a BMEP-NOx zeroth derivative cross correlation for pulsed throttle tests ......................... 41

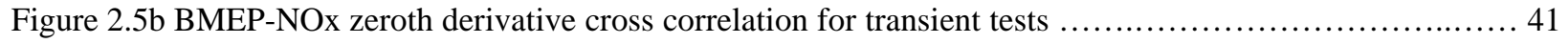

Figure 2.5c BMEP-NOx first derivative cross correlation for pulsed throttle tests ........................... 41

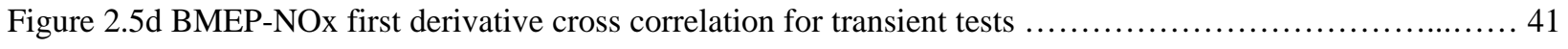

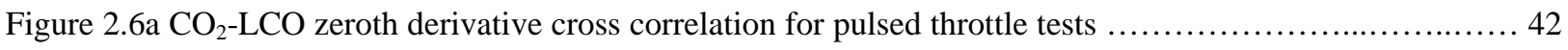

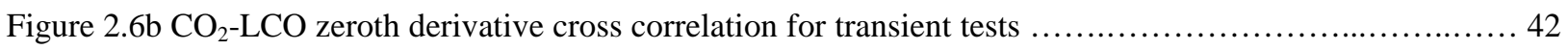

Figure 2.6c $\mathrm{CO}_{2}$-LCO first derivative cross correlation for pulsed throttle tests ............................. 42

Figure $2.6 \mathrm{~d} \mathrm{CO}_{2}-\mathrm{LCO}$ first derivative cross correlation for transient tests ................................ 42

Figure 2.7a LCO-THC zeroth derivative cross correlation for pulsed throttle tests ......................... 43

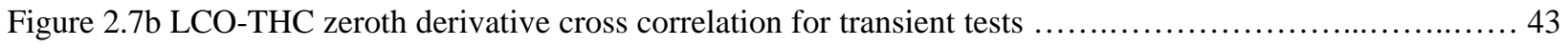

Figure 2.7c LCO-THC first derivative cross correlation for pulsed throttle tests $\ldots \ldots \ldots \ldots \ldots \ldots \ldots \ldots \ldots \ldots \ldots . \ldots \ldots$

Figure $2.7 \mathrm{~d}$ LCO-THC first derivative cross correlation for transient tests .............................. 43

Figure 2.8a FUEL-PM zeroth derivative cross correlation for transient tests .............................. 44 
Figure 2.8b FUEL-PM first derivative cross correlation for transient tests

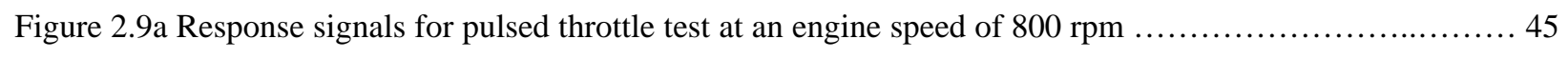

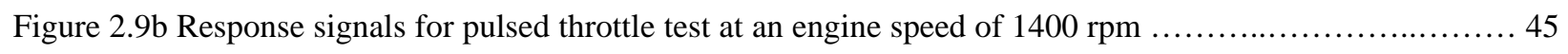

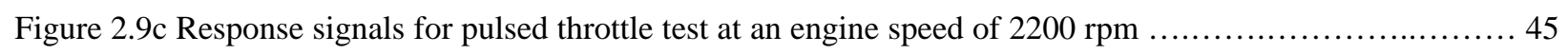

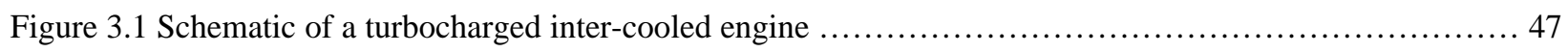

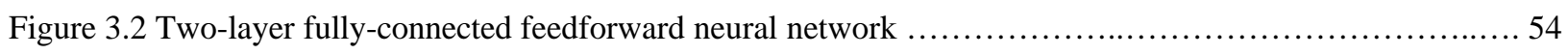

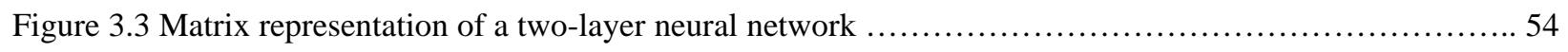

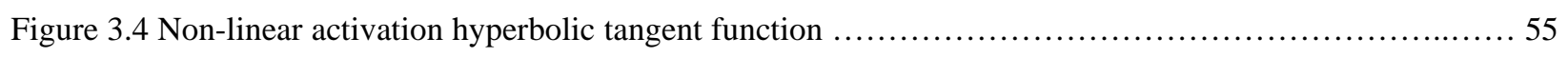

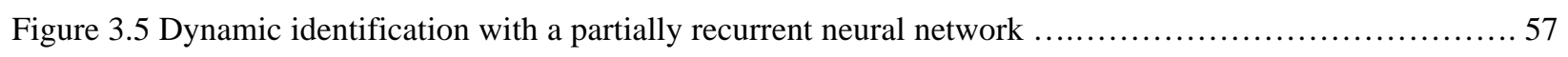

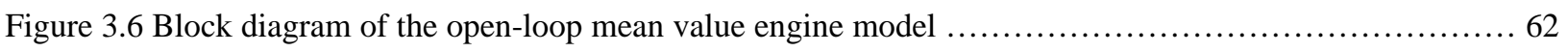

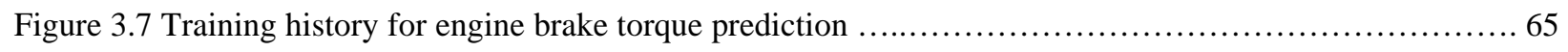

Figure 3.8 Measurement and series-parallel mode prediction of air flow rate over the FTP cycle $\ldots \ldots \ldots \ldots \ldots \ldots . . . . .73$

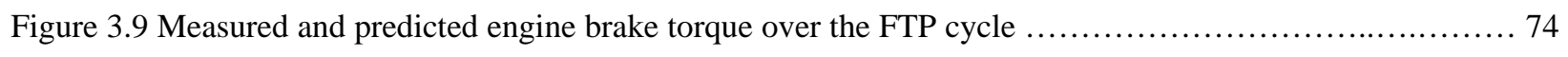

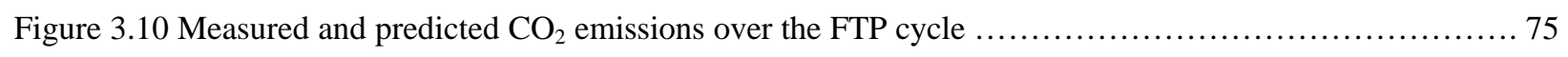

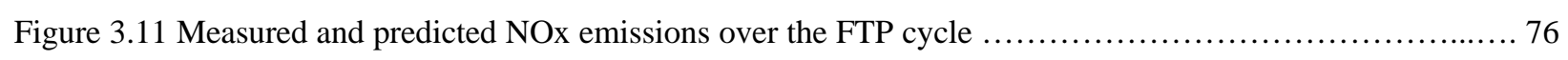

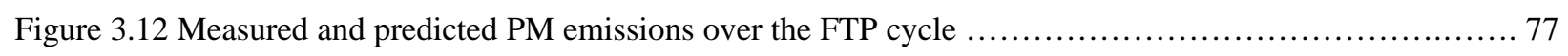

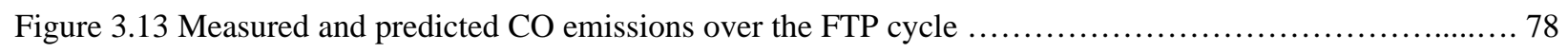

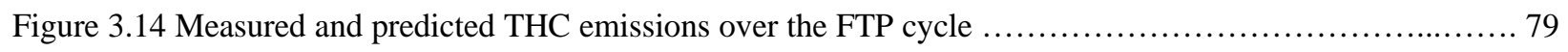

Figure 3.15 Measurement and parallel mode prediction of air flow rate over the FTP cycle $\ldots \ldots \ldots \ldots \ldots \ldots \ldots \ldots . . . . .60$

Figure 4.1a Speed history for desired and actual values over the FTP cycle $\ldots \ldots \ldots \ldots \ldots \ldots \ldots \ldots \ldots \ldots \ldots \ldots . . \ldots 4$

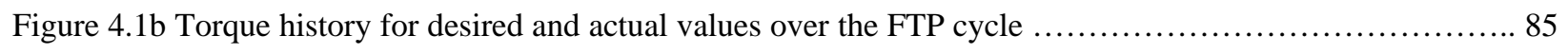

Figure $4.2 \mathrm{a}$ Speed history for desired and actual values over arbitrary cycle A.............................. 86

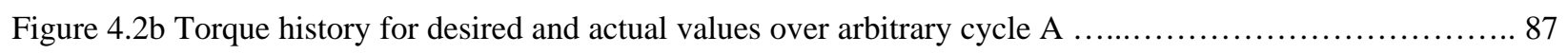

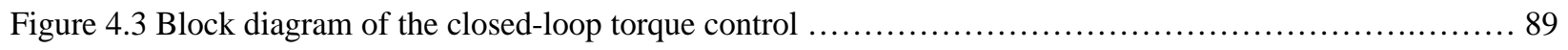

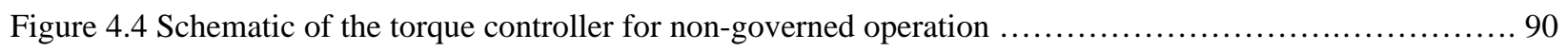

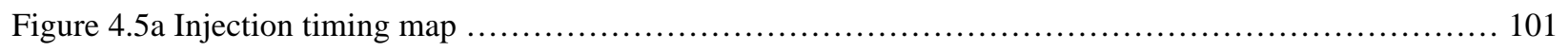

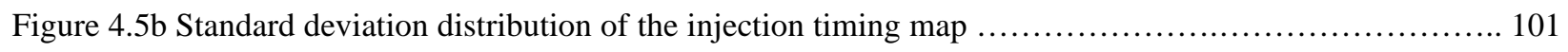


Figure 4.6a Injection pressure map

Figure 4.6b Standard deviation distribution of the injection pressure map

Figure 4.7 Measured and estimated injection timing over the FTP cycle 103

Figure 4.8 Measured and estimated injection pressure over the FTP cycle 104

Figure 4.9 Measured and estimated fuel mass flow rate over the FTP cycle 105

Figure 4.10 Combined neural networks layout for non-governed engine operation 106

Figure 4.11 Torque tracking performance over repeated pulsed torque test cycle for non-governed operation ... 107

Figure 4.12a Torque tracking performance over pulsed torque cycle at $800 \mathrm{rpm}$ for non-governed operation .... 108

Figure 4.12b Torque tracking performance over pulsed torque cycle at $1400 \mathrm{rpm}$ for non-governed operation ...109

Figure 4.12c Torque tracking performance over pulsed torque cycle at $2000 \mathrm{rpm}$ for non-governed operation ...110

Figure 4.12d Torque tracking performance over pulsed torque cycle at $2600 \mathrm{rpm}$ for non-governed operation ...111

Figure 4.13a Desired and actual torque over the FTP for PI torque control and non-governed operation 112

Figure 4.13b Desired and actual torque over the FTP for NN, lr=0.01, torque control non-governed operation .. 113

Figure 4.13c Desired and actual torque over the FTP for NN, $1 \mathrm{r}=0.10$, torque control non-governed operation .. 114

Figure 4.13d Desired and actual torque over the FTP for NN, lr=1.00, torque control non-governed operation .. 115

Figure 4.14a Brake specific fuel consumption at steady state operation 116

Figure $4.14 \mathrm{~b}$ Standard deviation distribution of the fuel consumption map 116

Figure 4.15a Brake specific $\mathrm{CO}_{2}$ emissions at steady state operation 117

Figure 4.15b Standard deviation distribution of $\mathrm{CO}_{2}$ emissions map

Figure 4.16a Brake specific NOx emissions at steady state operation

Figure 4.16b Standard deviation distribution of NOx emissions map

Figure 4.17a Brake specific PM emissions averaged over transient operation

Figure 4.17b Standard deviation distribution of PM emissions map 119

Figure 4.18a Brake specific THC emissions at steady state operation 120

Figure 4.18b Standard deviation distribution of THC emissions map ...... 120

Figure 4.19a Brake specific LCO emissions at steady state operation 121

Figure 4.19b Standard deviation distribution of LCO emissions map 121 
Figure 4.20a Total energy deviation versus regression slope and intercept over the FTP cycle

Figure 4.20b Positive energy deviation versus regression slope and intercept over the FTP cycle

Figure 4.20c Brake specific FC deviation versus regression slope and intercept over the FTP cycle 123

Figure 4.20d Brake specific THC emissions deviation versus regression slope and intercept over the FTP cycle 123

Figure 4.20e Brake specific LCO emissions deviation versus regression slope and intercept over the FTP cycle 123

Figure 4.20f Brake specific $\mathrm{CO}_{2}$ emissions deviation versus regression slope and intercept over the FTP cycle . 124

Figure 4.20g Brake specific NOx emissions deviation versus regression slope and intercept over the FTP cycle 124

Figure 4.20h Brake specific PM emissions deviation versus regression slope and intercept over the FTP cycle .. 124

Figure 4.21 Schematic of the torque controller for governed engine operation ............................ 125

Figure 4.22a Fuel flow rate characteristics for the two types of proportional governors considered ............. 129

Figure 4.22b Stepped accelerator position cycle at different engine speeds ............................... 129

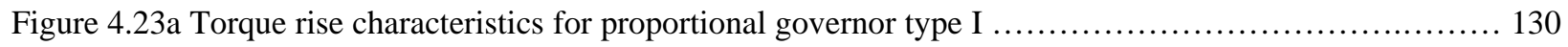

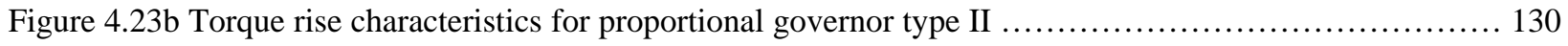

Figure 4.24a Carbon dioxide emissions for the two types of proportional governors considered ................ 131

Figure 4.24b Oxides of nitrogen emissions for the two types of proportional governors considered ............. 131

Figure 4.24c Particulate matter emissions for the two types of proportional governors considered .............. 131

Figure 4.24d Carbon monoxide emissions for the two types of proportional governors considered .............. 132

Figure 4.24e Total hydrocarbon emissions for the two types of proportional governors considered ............. 132

Figure 4.24f Fuel to air equivalence ratio for the two types of proportional governors considered ............... 132

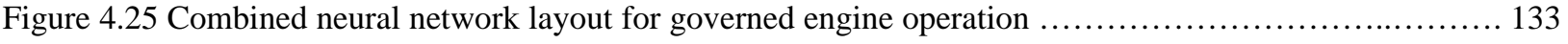

Figure 4.26a Tracking performance over pulsed torque transients with PI control for proportional governor I ... 134

Figure 4.26b Tracking performance over pulsed torque transients with PI control for proportional governor II . 134

Figure 4.27a Desired and actual torque over the FTP cycle with PI torque control for proportional governor I .. 135

Figure 4.27b Desired and actual torque over the FTP cycle with NN torque control for proportional governor I 136

Figure 4.28a Desired and actual torque over the FTP cycle with PI torque control for proportional governor II . 137

Figure 4.28b Desired and actual torque over the FTP cycle with NN torque control for proportional governor II 138

Figure 4.29 Block diagram for the closed-loop speed control 
Figure 4.30 Schematic of the engine speed controller

Figure 4.31a Governor desired speed as a function of accelerator position

Figure 4.31b Maximum torque fuel flow rate as a function of engine speed

Figure 4.32a Combined neural network layout for closed-loop speed control operation 145

Figure 4.32b Combined neural network layout for engine speed controller 146

Figure 4.33a Stepped accelerator position signal at different external loads 147

Figure 4.33b Speed tracking performance for the stepped accelerator test cycle

Figure $4.33 \mathrm{c}$ Error in speed tracking for the stepped accelerator test cycle

Figure 4.33d Fuel flow rate for closed-loop control of engine speed over the stepped accelerator test cycle 148

Figure 4.33e Engine torque for closed-loop control of speed over the stepped accelerator test cycle 148

Figure 4.34a Fuel flow rate characteristics for NN-based and type II governors 149

Figure 4.34b Engine torque characteristics for NN-based and type II governors 149

Figure 4.34c Fuel to air ratio characteristics for NN-based and type II governors 149

Figure 4.35a Carbon dioxide emissions for NN-based and type II governors 150

Figure 4.35b Oxides of nitrogen emissions for NN-based and type II governors

Figure 4.35c Particulate matter emissions for NN-based and type II governors 150

Figure 4.35d Carbon monoxide emissions for NN-based and type II governors 151

Figure 4.35e Total hydrocarbon emissions for NN-based and type II governors 151

Figure 4.36a Pulsed load cycle at different positions of accelerator 152

Figure 4.36b Speed tracking over pulsed load cycle at different positions of accelerator

Figure 4.36c Error in speed tracking over pulsed load cycle at different positions of accelerator

Figure 4.37 Schematic of the engine torque controller with embedded speed control

Figure 4.38 Combined neural network layout for torque controller with embedded speed control 155

Figure 4.39a Stepped torque cycle at different engine speeds 156

Figure 4.39b Torque tracking over stepped torque transients with PI control and embedded speed control ....... 156

Figure 4.40a Desired and actual torque over the FTP cycle with PI control and embedded speed control ... 157

Figure 4.40b Desired and actual torque over the FTP cycle with NN control and embedded speed control 158 


\section{LIST OF TABLES}

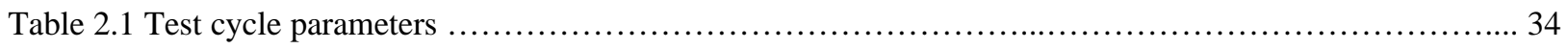

Table 2.2a Time shifts in seconds for maximum CC for pulsed throttle tests .............................. 36

Table 2.2b Time shifts in seconds for maximum CC for transient cycle tests ............................. 36

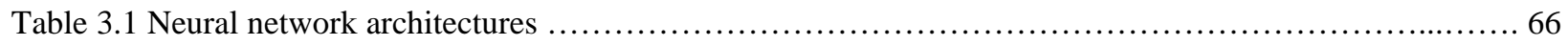

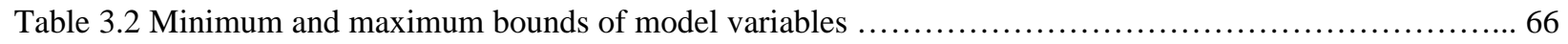

Table 3.3 Prediction integral error analysis for engine operating parameters ............................... 70

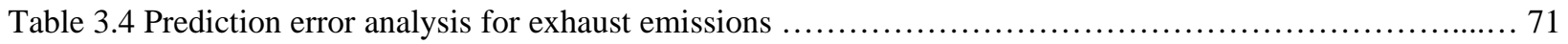

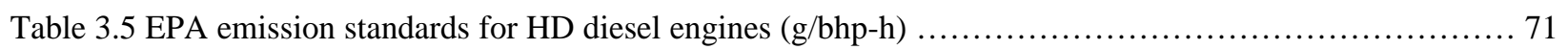

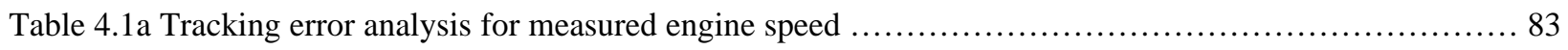

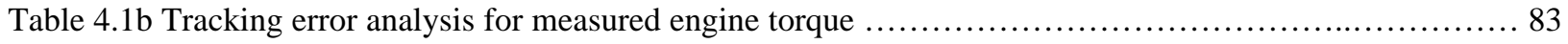

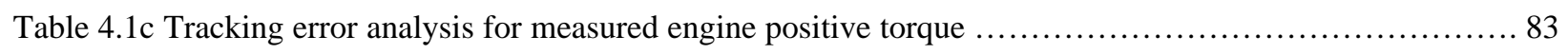

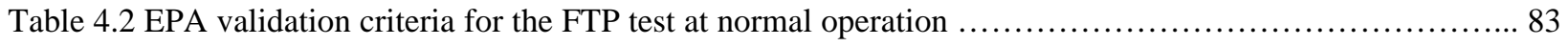

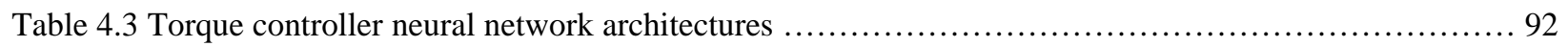

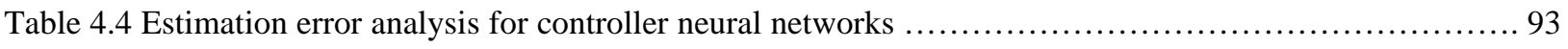

Table 4.5a Tracking error analysis over pulsed torque cycle for non-governed operation ...................... 95

Table 4.5b Tracking regression analysis over pulsed torque cycle for non-governed operation ................... 95

Table 4.6a Tracking analysis for positive torque over the FTP for non-governed operation .................... 97

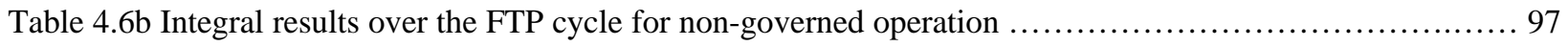

Table 4.7a Positive torque tracking error analysis over transient non-governed operation ..................... 98

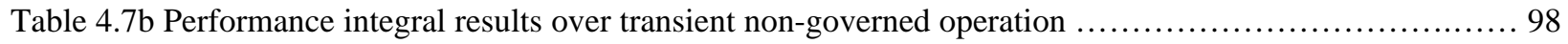

Table 4.7c Emissions integral results (g/bhp-h) over transient non-governed operation ........................ 98

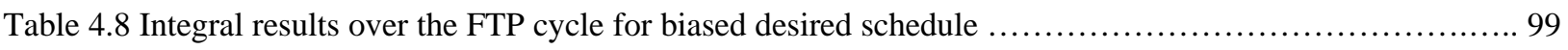

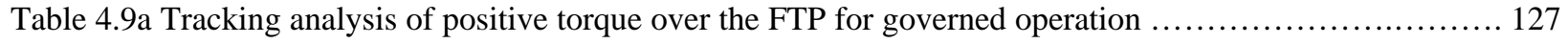

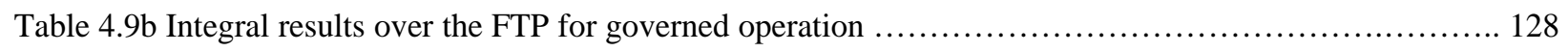

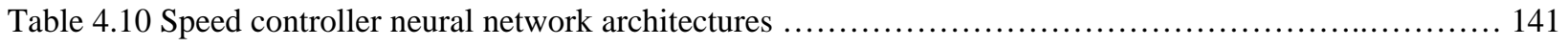

Table 4.11a Tracking analysis of positive torque over the FTP with embedded speed control ................... 154

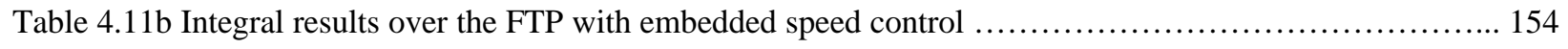




\section{English}

\section{NOMENCLATURE}

\begin{tabular}{|c|c|}
\hline a & neural network layer output vector \\
\hline $\mathrm{b}$ & neural network layer bias vector \\
\hline C & $y$-intercept of linear curve fit \\
\hline $\mathrm{CO}_{2}$ & carbon dioxide exhaust gas emissions \\
\hline $\mathrm{CO}, \mathrm{LCO}$ & carbon monoxide exhaust gas emissions \\
\hline $\mathrm{C}_{\mathrm{p}}$ & constant pressure specific heat \\
\hline $\mathrm{d}$ & time delay \\
\hline$d F$ & neural network layer Jacobian matrix \\
\hline$d^{2} F$ & neural network Hessian matrix \\
\hline e & error scalar or vector \\
\hline $\mathrm{E}_{\mathrm{i}}$ & $i^{\text {th }}$ pollutant mass flow rate \\
\hline $\mathrm{F}$ & non linear function \\
\hline $\mathrm{HC}, \mathrm{THC}$ & total hydrocarbon exhaust gas emissions \\
\hline I & moment of inertia or identity matrix \\
\hline $\mathrm{k}$ & time step \\
\hline $\operatorname{lr}$ & adjustable learning rate \\
\hline $\mathrm{m}$ & mass or linear best fit slope \\
\hline M & number of layers in a neural network \\
\hline $\mathrm{N}$ & engine speed or total number of data points \\
\hline $\mathrm{NOx}$ & oxides of nitrogen exhaust gas emissions \\
\hline $\mathrm{p}$ & neural network input vector \\
\hline $\mathrm{P}$ & gas pressure \\
\hline PM & particulate matter exhaust emissions \\
\hline$r 2$ & coefficient of determination \\
\hline $\mathrm{R}$ & gas constant \\
\hline
\end{tabular}




\section{Greek}

$\varepsilon$

$\gamma$

$\lambda$

$\mu$

$\eta$

$\Pi$

$\Gamma$

\section{Overstrike} number of neurons in a neural network layer neural network layer parameter sensitivity vector time or neural network desired output

temperature

control vector

volume

matrix of neural network layer weights

vector of the neural network weights and biases

state vector

intercooler effectiveness

ratio of gas specific heats

adjustable learning rate

adjustable training parameter

efficiency

pressure ratio

torque

- time rate of change

\section{Subscripts}

air or actual

C

compressor

d

displacement

e

engine or estimate

ex

exhaust

im

intake manifold 


$$
\begin{array}{ll}
t & \text { turbine } \\
t c & \text { turbocharger } \\
v & \text { volumetric }
\end{array}
$$

\section{Superscripts}

m

$\mathrm{T}$

\section{Acronyms}

ANN, NN

APS

ARB

ARMA

BMEP

CC

CI

CFR

$\operatorname{cov}$

CTM

DAS

DC

DI

DMUX

ECM

EEC

ENERGY

ENERGY+

EPA

FC neural network $\mathrm{m}^{\text {th }}$ layer

matrix transpose operator

artificial neural network

accelerator position signal

arbitrary

auto regressive moving average

brake mean effective pressure

cross correlation

compression ignition

code of federal regulations

coefficient of variation

counter timer module

data acquisition system

direct current

direct injection

de-multiplexer

engine control module

engine electronic control

integral brake energy expended over test cycle

integral brake positive energy expended over test cycle

Environmental Protection Agency

fuel consumption 


\begin{tabular}{|c|c|}
\hline FTP & Federal Test Procedure \\
\hline HD & heavy duty \\
\hline HEUI & hydraulically actuated electronically controlled unit injector \\
\hline HIL & hardware in the loop \\
\hline $\mathrm{Hz}$ & hertz \\
\hline IC & internal combustion \\
\hline ICP & injection control pressure \\
\hline IDI & indirect injection \\
\hline IW & input layer weights matrix \\
\hline LW & layer weights matrix \\
\hline MES & measured \\
\hline MOD & mode \\
\hline MUX & multiplexer \\
\hline OBD & on board diagnostic \\
\hline PI & proportional integral \\
\hline PID & proportional integral derivative \\
\hline ppm & parts per million \\
\hline $\mathrm{rpm}$ & revolutions per minute \\
\hline RPT & rapid prototyping \\
\hline SI & spark ignition \\
\hline SIM & simulated \\
\hline SOI & start of injection \\
\hline $\operatorname{stdv}$ & standard deviation \\
\hline TEOM & tapered element oscillating microbalance \\
\hline TORQ & engine brake torque \\
\hline WOT & wide open throttle \\
\hline
\end{tabular}




\section{CHAPTER 1}

\section{Introduction}

\subsection{Background}

Increasingly stringent emissions legislation and demands for improved fuel economy and engine performance has resulted in a wide expansion of the functions that have to be performed by engine management electronics. This has mandated the need for advanced control algorithms and complicated the engine calibration procedure. Automation and model-based calibration procedures are becoming essential to reduce the cost and time of development, which conventionally involve elaborate sweeps of the control variables on a transient engine test bed (Voigt, 1993; Schmitz et al., 1994; Onder and Gerring, 1995). Moreover, dynamic system simulation is paramount for rapid prototyping (RPT) of control strategies for embedded systems where the basic procedure involves constructing a dynamic model, synthesizing a control law, and analyzing the closed-loop performance and robustness through simulation (Lukich et al., 1995). In an RPT environment, shown in Figure 1.1, the functionality of a dynamic model includes (Weeks and Moskwa, 1995):

(1) development of control algorithms without the hardware constraints and software complexities of running in real-time,

(2) evaluation of actual engine controllers or actuators using hardware in the loop (HIL) simulation, shown in Figure 1.2, where actual engine and actual sensors are replaced with real-time simulators connected to the real hardware components, and

(3) development of model-based controls where the system model is embedded into a real-time control algorithm. 


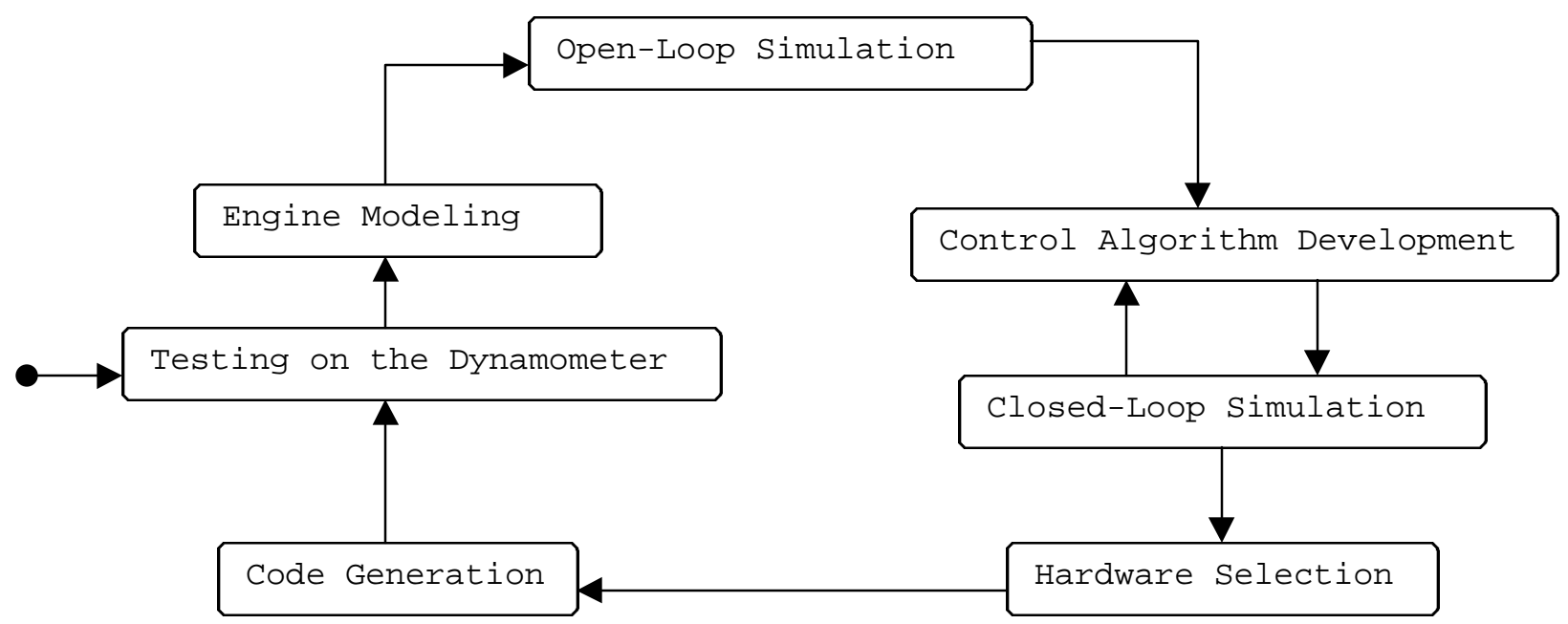

Figure 1.1 Control algorithm synthesis in a rapid prototyping environment

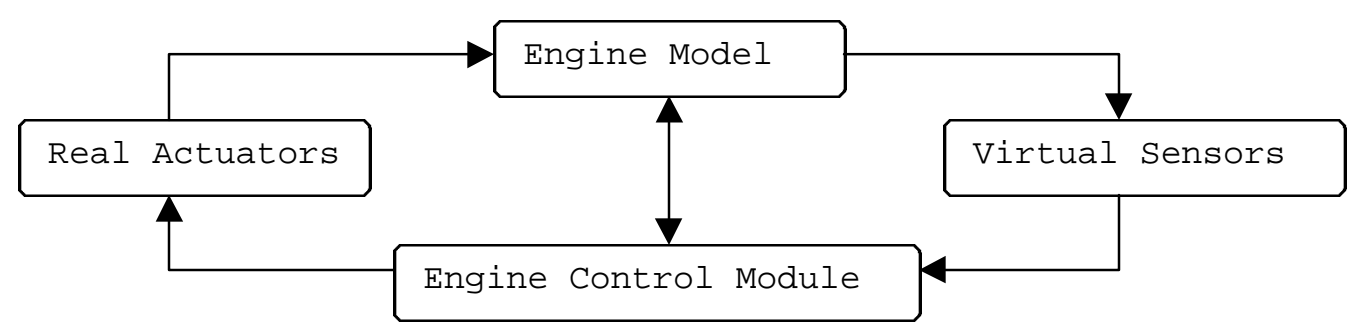

Figure 1.2 Hardware-in-the-loop

For simulation and control purposes, mean value models provide adequate characterization of the engine dynamic behavior. They describe the time development of the main engine variables (e.g. speed and manifold pressure) on time scales slightly longer than that required for a single engine cycle (Hendricks and Sorenson, 1990). Development of subsystem models can be predominantly either theoretical or experimental. Theoretical modeling is based on physical laws of mass and energy conservation. Phenomenological description with a high level of empiricism is used to model those processes which are not physically understood. The high complexity of this modeling approach and its computational requirement makes it unsuitable for real-time simulation. 
Experimental modeling is based on system identification methodologies applied to measured input and output signals.

Coupling dynamic engine simulation with emissions prediction provides an invaluable cost effective analysis tool. It allows for: (1) evaluating the impact of the control algorithm on transient exhaust emissions, and (2) identifying engine operating conditions that contribute most to the overall emission levels through modal analysis (Hales and May, 1986). Also, it is possible to alter engine specifications (e.g. the maximum torque map) and evaluate their effect on engine performance and emissions. Further, if the engine control variables are exercised over a wide operation range during the system identification phase, the cycle simulation capabilities can be greatly extended, for example in the development of new calibration maps to meet a desired emissions standard. The effectiveness of this approach in reducing the time and cost of development of control algorithms is clearly evident (Isermann et al., 1998; Stobart et al., 1998). One immediate application of an emissions prediction module is its integration for on boarddiagnostics (OBD) (Atkinson et al., 1998a). For example, an on board virtual NOx sensor could be an essential tool to meet the regulatory monitoring requirements of an OBD system for diesel engines. NOx emissions above (say) 1.5 times the standard would trigger the malfunction indicator lamp.

Since internal combustion engine dynamics and emissions formation are time variant, multidimensional, and highly nonlinear, the identification and control of such a system can be attempted with a learning scheme. Artificial Neural Networks (ANN) offer a very attractive approach since an ANN identification scheme does not require detailed knowledge of either engine performance governing equations or the combustion kinetics of emissions formation. 
Transient testing of internal combustion (IC) engines is an increasingly important part of engine development work, particularly in medium and heavy-duty (HD) compression ignition (CI) engines which are mostly open-loop controlled, thus requiring elaborate engine calibration to be conducted on a transient engine dynamometer. Increasingly stringent emissions legislation and demands for improved fuel economy and driveability requires more work in research and development to optimize transient engine operation and control. This result in a growing need for accurate and repeatable transient control of engines on test beds (dynamometers). An example of the expected benefits is the potential reduction in particulate matter (PM) emissions of $20 \%$ (for a low rated engine) to $70 \%$ (for a highly rated engine) if 'spikes' in transient PM emissions are eliminated during acceleration phases on the FTP cycle (Watcher, 1990). Test bed control systems must track the pre-specified speed and torque schedule of the test cycle. Ideally, speed control is accomplished using the dynamometer's own controls and torque is controlled via the engine fueling (Clark et al., 1984). Conventionally, three-term, proportional-integral-derivative (PID) feedback controllers are used because of their low computational requirement and simplicity of tuning (Noble et al., 1988). However, their main drawbacks are poor performance compared with that of modern control theory implemented on the same hardware and that optimal performance is not necessarily guaranteed under all operating conditions. This problem is more pronounced in a research and development environment where engine calibrations and test cycles are constantly modified. On the other hand, modern control techniques allow for (1) multivariable control, (2) incorporation of dynamic models of the system, and (3) control action resulting from direct minimization of a cost function. These techniques have the potential not only to realize better tracking performance but also to minimize the time required to achieve a validated test cycle. 
The aim of the current investigation is twofold. For a modern diesel engine, firstly develop and validate a neural network-based mean value model capable of predicting engine dynamic behavior as well as exhaust gas emissions. Secondly, develop and validate, through simulation, neural network-based engine control. The latter objective involves the development of a torque controller for the purpose of tracking a pre-specified arbitrary torque schedule. The cases of nongoverned as well as speed governed engine operation are considered. Also, a neural network based all-speed governor is developed and embedded in the torque control loop.

This dissertation proceed with a literature review detailing the state of the art mean value modeling of IC engines, neural-network applications in internal combustion engines for system identification and control, and the transient control of heavy duty compression ignition engines on test beds. Experimental data gathering procedures are then described followed by the development and validation of the mean value engine model. The development of the neural network-based engine control is presented and validated through simulation. Finally, this dissertation concludes with an overview of the accomplished research efforts and future recommendations. 


\subsection{Literature Review}

Firstly, the mean value approach is described and its implementation to IC engines for the

purpose of system identification and control is reviewed. Secondly, the application of artificial neural networks to internal combustion engines is reviewed with an emphasis on performance and exhaust emissions prediction. Finally, the application of modern control theory to the problem of tracking a pre-specified test schedule on a transient test bed is reviewed.

\subsubsection{Mean Value Modeling of IC Engines for Control Algorithm Development}

Mean value engine models represent an intermediate level of modeling between large complex cyclic simulation models and simplistic linear transfer function models. The modeling effort is aimed at providing a simple yet adequate characterization of the engine dynamic behavior on time scales slightly longer than those required for a single engine cycle, which makes them suitable for model-based control algorithms. Development of mean value models can either be predominantly based on large cyclic simulation, or based on actual engine testing experiments on a transient test bed. For the latter, prediction accuracy is primarily dependent on selecting the correct physical variables and their various dependencies.

On the time scale considered by the mean value approach, engine variables' dependencies are classified as either time dependent or instantaneous (Hendricks and Sorenson, 1990). Differential equations are used to describe the time history of engine variables or states such as speed and manifold air pressure, which require more than a few engine revolutions to reach equilibrium. Instantaneous relationships are those which describe the dependence of static internal variables on dynamically developing engine states, for example thermal and volumetric efficiencies as functions of state variables. 
One must distinguish between two approaches to model the system dynamics. In the first, graybox approach, differential equations based on conservation laws are retained and static functional relationships are approximated. In the second approach, the system is treated as a black box and an identification scheme is used to characterize the system transient behavior using only measured input and output data. The experimental burden is greatly reduced in the gray-box approach by virtue of its static 'steady state' nature. Further, even when employing a black-box approach, physical considerations are necessary to guide the transient experimental procedure and to select the appropriate state variables, their dependencies, and associated time delays.

In its simplest form, the engine state vector consists of engine speed and manifold air pressure for the case of a naturally aspirated throttle-less direct injection IC engine. The engine control vector consists of fuel mass rate and injection timing (CI) or spark timing (SI), and engine load is treated as an external disturbance. The system dynamics are described by mass conservation applied to the intake manifold and energy conservation applied to the engine crankshaft. Two instantaneous 'static' engine variables, volumetric and thermal efficiencies, are expressed in terms of the engine state and control variables. In this formulation, energy losses in pumping and friction are accounted for in the thermal efficiency term or they have to be identified as static variables.

Employing a throttle, as a mean of load control, adds one more control variable (throttle position) and one more static variable (discharge coefficient across the throttle). If direct injection is replaced by port or central injection, fuel film mass flow is added to the state vector and fuel film fraction and evaporation time constant are two more static variables, needed to describe the fuel film dynamics.

Furthermore, employing a turbocharger complicates the model because it couples the engine's 'breathing' ability with its combustion efficiency. The state vector is augmented by one more 
variable, turbocharger speed, which is governed by energy conservation applied to the turbocharger shaft. Also several static variables need to be considered (air mass flow rate, torque, and outlet gas temperature), which depend on the steady state characteristics (pressure ratio, shaft speed, and inlet gas temperature) of the turbocharger's compressor and turbine. Implicit in this formulation is the need to estimate the gas pressure and temperature in the exhaust manifold. These are either calculated from state and control variables such as the air and fuel flow rates, or augmented to the state vector and conservation of mass and energy equations are applied to the exhaust manifold to characterize their dynamic behavior.

In the following paragraphs, different approaches to modeling turbocharged diesel engines, in the mean value sense, are presented as reported by several researchers. The emphasis is placed on the variables used to construct the engine state vector and their governing differential equations, elements of the control vector, the static variables considered and their functional dependencies', and the accuracy of the reported predictions.

Jensen et al. (1991) developed a five-state mean value model for a 1.6 liter turbocharged indirect injection diesel engine. The state vector consisted of intake manifold pressure and temperature, engine and turbocharger speeds, and exhaust manifold pressure. The system dynamic behavior was governed by mass conservation applied to the intake and exhaust manifolds, energy conservation applied to the engine and turbocharger shafts, and intake manifold energy conservation. The control vector consisted of fuel rack position index. The static variable vector included the compressor pressure ratio and efficiency, turbine mass flow rate and efficiency, waste gate position, indicated thermal efficiency, friction power loss, volumetric efficiency, and exhaust manifold temperature. They modeled compressor pressure ratio and efficiency as functions of flow rate and inlet Mach number. Turbine efficiency was expressed as a function of the 
turbocharger speed and the ratio between the isentropic expansion velocity and the turbine tip speed, the turbine mass flow rate as a function of pressure ratio and the turbocharger speed. They modeled indicated thermal efficiency as a function of air to fuel ratio and engine speed, volumetric efficiency as a second order polynomial of engine speed, and friction power loss as a function of mean piston speed and intake manifold pressure. Exhaust gas temperature was expressed as a function of air to fuel ratio, intake manifold temperature, and engine speed. The engine was tested on a dynamic test bed and the engine variables were measured at a rate of $10 \mathrm{~Hz}$ which included engine speed and load, temperatures and pressures before and after the turbine and the compressor, air flow rate, and turbocharger speed. Prediction errors were reported within $\pm 10 \%$ of the measured values of the engine variables for steady state as well as transient operation.

Hendricks (1986, 1989) developed a two state (engine speed and manifold pressure), one control variable (rack position index) model of a large turbocharged inter-cooled two-stroke diesel engine. The engine dynamic behavior was governed by energy conservation applied to the engine and the turbocharger shafts. Hendricks derived an expression relating manifold pressure to turbocharger speed thus eliminating the need to consider turbocharger speed as an independent state variable. The model did not account for the time constants for the scavenging and exhaust manifolds as these were much smaller than the effective time constant involved in speeding up the turbocharger for that particular engine configuration. Static variables vector included indicated thermal efficiency as a function of air to fuel ratio, scavenging ratio as a function of engine speed and manifold pressure, compressor flow rate as a function of its pressure ratio, and friction power loss as a function of engine speed. Turbocharger efficiencies were considered constant since they did not vary along a steady state engine operating line and exhaust gas temperature was expressed as a function of manifold temperature and air to fuel ratio (Hendricks, 1986). Static variables 
dependencies were calculated based on the results of a comprehensive crank-based engine simulation program used to calculate engine variables at 20 points within the engine operation envelope. For rapid step changes in engine load, accurate predictions of manifold pressure and engine speed were reported for a number of engines tested using a transient dynamometer. Hendricks deduced that the engine dynamic behavior was controlled by two time constants: one due to the inertia of the engine crankshaft and associated masses and another directly proportional to the turbocharger inertia and inversely proportional to the compressor air mass flow rate. Thus the turbocharger boost lag behavior (large time delay at low engine speeds which becomes smaller at higher engine speeds) was correctly identified.

Hendricks and Poulsen (1986) used the two-state engine model described above to construct an extended Kalman filter acting as a non-linear engine observer. The dynamic state matrix of the linearized model was used for the required online solution of the Riccati equation. Gaussian white noise was assumed for both states and measurements. State estimates were reported within three times the noise standard deviation and the filter performance remained stable and unbiased for 5\% offsets artificially introduced into the measurements.

Watson (1984) presented a very detailed mean value model with a basic time step of 0.05 seconds coupled with a crank-based in-cylinder power-cycle calculation for the purpose of dynamic simulation of turbocharged diesel engines. The system dynamic behavior was described by applying conservation laws (mass and energy) to the cylinders and manifolds, and the engine and turbocharger shafts. An empirical combustion model was used to characterize ignition delay as a function of fuel cetane number and gas state at start of injection, and premixed and diffusion mass fractions as functions of ignition delay, engine speed, and fuel to air ratio. Friction power loss was modeled as function of mean piston speed, maximum cylinder pressure and engine oil 
temperature. Volumetric efficiency was expressed in terms of engine speed and intercooler effectiveness and pressure drop as functions of air mass flow rate. The turbocharger compressor and turbine characteristics (efficiency, mass flow rate, and torque) were modeled as functions of turbocharger speed and pressure ratio. Smoke opacity was correlated with engine speed, fuel to air ratio, and fuel premixed burning fraction. Accurate overall predictions of steady state and transient performance were reported for a truck engine and another highly rated inter-cooled engine.

Gissinger et al. (1989) developed a $30^{\text {th }}$ order mean value model of a turbocharged six cylinder diesel engine. Mass and energy conservation was applied to each cylinder separately which resulted in the increased number of state variables required to describe the system dynamic behavior. Closed-loop performance of several all-speed governors, ranging from basic PID to direct adaptive predictive, was briefly reported.

Tsai and Goyal (1986) developed a one state variable (engine speed) mean value model for a turbocharged diesel engine. The engine speed dynamics were governed by energy balance applied to the crankshaft. The engine brake torque was expressed as a function of cycle specific fuel flow rate, engine speed, and air to fuel ratio. The airflow rate across the compressor, boost pressure, and the turbocharger lag were modeled as functions of engine speed and fuel quantity. The model predictions were in good agreement to those from a more extensive thermodynamic simulation program during speed and load transients.

Zhang et al. (1989) developed a fifth order ARMA (Auto-Regressive Moving-Average) model to describe the speed dynamics of a turbocharged diesel engine as a function of fuel flow rate, control input, engine load, and disturbance input. Model parameters were estimated from leastsquares analysis of engine speed and load at steady state operation. A pole assignment technique 
was used to design a lag-lead controller to track the speed trajectory of minimum fuel consumption at different engine load settings. Good tracking performance with reduced fuel consumption was reported for different loading conditions.

Kao and Moskwa (1995a) presented a three state variable (manifold pressure, engine and turbocharger speeds) mean value model of a turbocharged diesel engine. System dynamic behavior was governed by mass conservation applied to the intake manifold and energy conservation applied to the engine crankshaft and the turbocharger shaft. Compressor and turbine efficiencies and mass flow rates were expressed in terms of turbocharger speed and its respective pressure ratios. The pressure and temperature variations across the inter-cooler were functions of air mass flow rate. Indicated thermal efficiency was expressed in terms of engine speed and air to fuel ratio. Volumetric efficiency and friction power loss were functions of engine speed. The engine temperature rise (exhaust minus intake manifold temperatures) was expressed in terms of air to fuel ratio and engine speed. The exhaust gas pressure was modeled as a function of intake manifold pressure and temperature, engine temperature rise, and mass flow rate. In order to simulate the transient temperature response in the exhaust manifold, the exhaust gas temperature was estimated by adding the dynamic engine temperature rise to the intake manifold temperature with a delay (the exhaust manifold time constant). A fourth order Runge-Kutta integration scheme was used to solve the governing first order differential equations at a time step of 0.02 seconds. The capability of the overall model to predict engine performance was demonstrated through simulation.

Kao and Moskwa (1995b) used their mean value model to design a sliding mode nonlinear controller to track a prescribed engine speed trajectory in the presence of load disturbance with the fuel mass as the control variable. The controller design included a mean value model of the 
engine speed dynamics and load estimation using a nonlinear sliding observer. Simulation results demonstrated better tracking ability than a linear PI counterpart and good robustness to both modeling error and measurement noise. 


\subsubsection{Mean Value Modeling of Exhaust Gas Emissions}

Mean value modeling of exhaust gas emissions is an intricate part of the engine calibration procedure where the goal is to optimize control parameters, such as start of injection, injection pressure and duration, to minimize fuel consumption subject to the constraint of meeting a statutory emission standard. It is a common procedure to tailor the control parameters such that exhaust emissions meet a desired standard on the regulatory test cycle with the best possible fuel economy. For heavy-duty engines, a transient test cycle is used in the US (the Federal Test Procedure), while steady state test cycles are used in Europe (EC R49 13 mode) and in Japan (D13 mode) (Mori, 1997). The testing cycle is defined in terms of a time sequence of load versus engine speed points. Transient cycles can be decomposed to a set of key steady state points based on a histogram of the various load/speed points and operating points contributing most to the emissions levels. An example for this approach is the decomposition of the US FTP test cycle to the AVL 8 mode steady state test cycle (Aufinger and Cichocki, 1993). Elaborate sweeps of the control variables can be conducted at each point and the optimal control problem of minimizing fuel consumption subject to a set of emission constraints may be solved (Schmitz et al., 1994).

For the application of the above procedure, the sensitivities of the exhaust emissions to engine state (load and speed) and swept control variables have to be calculated. Regression analysis can be used to determine an appropriate parametric model of emissions and fuel consumption (Mencik and Blumberg, 1978). Further, the steady state based-models can be used to predict the transient emissions over the dynamic test cycle in order to evaluate the effectiveness of the determined calibration in meeting the desired emissions standard. The significance of this procedure, which has been common practice in the engine research and development community, is twofold. Firstly, black-box models are capable of providing accurate predictions of engine-out exhaust emissions. 
Secondly, steady state-based models are capable of projecting transient operation emissions. The significance of the latter is paramount for a mean value model since it is assumed that emissions formation can be treated as a series of static non-linearities with no appreciable time dependence on the considered time scales.

Although an extensive review was conducted on the large body of literature relating the influence of engine state and control variables on emissions from direct injection turbocharged CI engines (the engine of choice for this study), it was felt unnecessary to include this effort in this presentation. This is simply because a black-box model approach was adopted. However, some specific results and trade-off relationships will be stated and appropriately referenced when required. 


\subsubsection{Application of Neural Networks to IC Engines}

The basic building block of the biological brain is the neuron (a nerve cell), which communicates information to and from various parts of the body. The nervous system is composed of a massive highly interconnected network of neurons, which are capable of storing experiential knowledge and making it available for use. Artificial neural networks (ANN) are simple mathematical abstractions of the biological brain activities where knowledge is acquired through learning. In its simplest form, the functional operation of the model neuron consists of adding the weighted-sum of its input signals, summing an internal threshold value, and passing the result as an argument to an activation function to generate an output signal. In general, ANN architecture consists of one or more layers of neurons connecting input and output vectors. Layers other than the input and output layers are termed hidden layers, whose output signals are the input signals to the succeeding layer. A learning algorithm modifies the weights and thresholds of the ANN architecture in an orderly fashion, typically gradient-based, so as to minimize a pre-established cost function. A comprehensive treatment of ANN formulation is available in the texts by Hagan et al. (1996), Haykin (1994), Simpson (1990), and the review article by Lippmann (1987).

Considering the task of identification and control of non-linear systems, an ANN approach offers the following useful properties:(1) the ability to map non-linear input-output associations through supervised learning, (2) an inherent representation of a multivariable system, (3) a realtime built-in capability to adapt to time-varying systems, and (4) fault tolerance due to its inherent parallel representation.

Artificial Neural Networks (ANN) offer a very attractive approach to the problem of identification and control of internal combustion engine dynamics and exhaust emissions formation which are time variant, multidimensional, and highly non-linear processes. Furthermore, 
an ANN identification scheme does not require the detailed knowledge of either engine performance governing equations or the combustion kinetics of emissions formation. In the following paragraphs, the application of neural networks to IC engines, as reported by several researchers, is presented.

Ayeb et al. (1998) outlined two approaches to NN-based SI engine modeling for the development of control algorithms. In the first gray-box approach, mathematical models that identify the system dynamics are retained and static functional relationships are approximated using a static NN. For example, the throttle valve discharge coefficient as a function of throttle angle, volumetric efficiency as a function of engine speed and manifold air pressure are used. In the second approach, each sub-model is treated as a black box and a dynamic NN is used to identify the system transient behavior using only measured I/O-data. Two architectures of dynamic NNs were proposed; either a static feed forward NN with external dynamic characteristics (time delay neural nets) or networks with internal dynamic characteristics (recurrent networks). Ayeb et al. used single layer static NN to approximate the static dependence of airflow rate into the intake manifold based on throttle position and engine speed. Another static NN was used to approximate the mass airflow out of the intake manifold based on manifold pressure and engine speed. The two networks were augmented with a dynamic model of the intake manifold and were successfully used to predict the manifold gas pressure. In an alternative approach, a time delay single hidden layer, 8 neuron, NN was used to predict the manifold pressure directly from present step values of engine speed and throttle angle, and previous step values of throttle angle and manifold pressure. The NN was trained off line using sweeps on engine speed and throttle position and good generalization capability was reported over dynamic transients not included in the training. 
Atkinson et al. (1998a) implemented a partially recurrent NN architecture to predict engine output torque and engine exhaust gas emissions for a turbocharged 10 liter DI diesel engine as well as a naturally aspirated 1.9 liter spark ignition engine. The input vector included a receding history window of measurable engine operating parameters (e.g. intake air temperature and pressure, engine speed, engine coolant and oil temperatures). The receding window history (10 seconds) for the CI engine was longer than that for the SI engine (5 seconds) to account for the transient response of the turbocharger. The engines were exercised through wide ranges of their performance envelopes and non-ordered sweeps on the control parameters were conducted. Accurate predictions were reported over highly transient test cycles for both engines.

Hanzevack et al. (1997) developed three neural networks, which use measurable engine operating parameters, to predict engine output power, fuel pressure, and stoichiometric index as well as exhaust gas emissions for an air-cooled general aviation aircraft engine. A two-hidden layer architecture was used for the first and second nets. The third net featured a competitive network with two expert nets and a sliding history window of engine operating parameters. A gating net employing a hidden layer was used to ensure that the classification problem was solvable. Under normal engine operating temperature, good predictions were reported over transient test schedules.

Ludwig and Ayoubi (1995) implemented a two-layer NN with a dynamic perceptron model (DPM) to identify the non-linear dynamics of a turbocharger in a diesel engine. The DPM employed a second order digital filter, which was applied to the weighted sum of the input vector before passing the result as an argument to an activation function to generate the output signal. The NN architecture consisted of two inputs (engine speed and fuel injection mass), 5 dynamic neurons in the hidden layer, and the manifold pressure as system output. A transient test cycle was 
conducted on a dynamic test stand where the engine was exercised over a speed and load (fuel mass injected) variant cycle one input at a time while the other was set to its static value. Accurate identification of manifold pressure was reported over a transient test cycle not included in the training procedure.

Isermann et al. (1998) applied a local basis function NN for identification of a truck diesel engine exhaust turbocharger. The manifold charging pressure was modeled as the output of a nonlinear second order multiple input $\mathrm{NN}$ with current and previous values of engine speed and fuel injection mass rate, and recurrent values of charge pressure over two time steps as inputs. Accurate prediction of charging pressure was reported over transient operation.

Salam and Gharbi (1996) implemented a single hidden layer NN to model the forward dynamics of an SI engine using a mathematical model of the system based on steady-state data. Inputs to the NN consisted of a state vector (speed and manifold air pressure), a control vector (spark timing and throttle position), system disturbances, and the previous step state vector. An inverse dynamics NN controller was developed to track a reference model state and satisfactory tracking dynamics was demonstrated.

Scaife et al. (1993) used a single hidden layer with non-linear activation for fault recognition of the operation of a turbocharged HD diesel engine. A computer simulation was used to train the $\mathrm{NN}$ on the engine behavior with various faults over its entire operating envelope. The simulated faults included a fouled intercooler, an exhaust blockage, leaks in the inlet and exhaust manifolds, and an exhaust valve leak. The network architecture consisted of 17 inputs that represent operating parameters (e.g. engine speed, turbocharger speed, manifold pressures and temperatures), 13 neurons in the hidden layer, and six output parameters that represented the engine's state of health. The NN was trained in a batch mode using a back-propagation algorithm. 
The network responded successfully to real engine data when tested for healthy as well as faulty operation.

De Nicolao et al. (1996) approximated the static non-linear dependence of the volumetric efficiency on engine speed and manifold air pressure using a radial basis function neural network and a two-layer perceptron network with non-linear activation. The training data was provided through steady state sweeps on engine speed, torque, and spark timing for a 1.6 liter SI engine.

Nelson et al. (1996) trained a neural network to approximate the characteristics of a centrifugal compressor in the context of a turbocharged diesel engine simulation. The NN architecture consisted of a single non-linear hidden layer and was trained using the Levenberg-Marquardt algorithm. Adding an additional input parameter, termed the design parameter, a NN was trained to approximate an entire family of compressors. The versatility of the NN-based approach was demonstrated via matching studies performed for intercooled and non-intercooled configuration of a truck type diesel engine.

Leonhardt et al. (1995) used a radial basis function neural network to estimate injection fuel mass and injection timing from measured in-cylinder gas pressure for a 1.6 liter, turbocharged diesel engine. Input parameters to the NN were the center of gravity point and peak point of a difference pressure signal (calculated by subtracting a reconstructed motored pressure signal from the fired pressure signal), and engine speed. The $\mathrm{NN}$ was trained on static data points generated from sweeps on fuel injection mass, injection timing, and engine speed. The NN reconstruction performance demonstrated a less than $10 \%$ deviation for static as well as dynamic operation.

Müller and Hemberger (1998) estimated the location of the 50\% burnt mass fraction from measured in-cylinder gas pressure using a totally connected feed forward network in an SI engine. Steady state sweeps on engine load, speed, and ignition timing were used for off-line training of 
the NN, using back-propagation algorithm. A pruning algorithm was used to reduce the on-line computational effort based on removing redundant neurons and links. A mean error of 0.05 degrees was reported for the test set. In an effort to develop a neural controller for adaptive ignition control, a system identification $\mathrm{NN}$ was trained to predict the point of $50 \%$ burnt mass from measurable engine parameters (ignition timing, air mass flow rate, and engine speed). A single hidden layer $\mathrm{NN}$ architecture with 5 neurons was trained using a real time recurrent learning algorithm with static and dynamic derivatives. Acceptable agreement was reported with measured data over a transient test cycle. Through simulation, the NN-based adaptive ignition controller was reported to regulate the point of the $50 \%$ burnt mass at a desired location.

Lenz and Schroeder (1996) implemented a general regression NN architecture containing 150 neurons to identify inlet manifold dynamics using measurable engine operation parameters (inlet manifold air pressure and temperature, engine speed, and mass air flow rate through throttle) in an SI engine. Mean value modeling was used to identify unknown static non-linearity (volumetric efficiency for the case at hand) embedded in the middle of the plant, in what they termed local identification. Measured manifold air pressure was used to create an error signal able to adapt the $\mathrm{NN}$ with guaranteed stability (due to local activation), proven using Ljapunov's stability theory. They argued that back-propagation-based algorithms were not suitable because of a lack of stability proof for gradient descent over a complex, highly dimensional error surface leading to local minimum or instability.

Shayler et al. (1996) used a single hidden layer NN with 5 neurons to estimate the transient fuel compensation required for a stoichiometric mixture for an SI engine employing port fuel injection. The inputs to the NN included cylinder air charge mass, coolant temperature, and estimated fuel film mass. Standard back-propagation was used to train the NN using an error signal calculated 
from the measured air-fuel ratio using a wide range oxygen sensor. The approach demonstrated the ability of NN-based approximation to replace a large number of look-up tables required to calculate the necessary transient fuel compensation. Similarly, Lenz and Schroeder (1997) proposed a general regression $\mathrm{NN}$ approach to identify the non-linear dynamics of port fuel injection process due to the wall-wetting effect using a wide range lambda sensor to provide the adaptation error signal.

Lenz and Schroeder (1998) demonstrated, through simulation, a general regression NN-based adaptive mapping technique to realize a desired air-fuel ratio using manifold air pressure and engine speed for DI engine concepts. They provided a mathematical proof for stability and parameter convergence of their on-line adaptation scheme. Their approach accounted for static non-linearity caused by the volumetric efficiency and injection efficiency.

Asik et al. (1997) used a two-hidden layer NN with 8 sigmoidal nodes in each layer to estimate the air-fuel ratio for a 4.6 liter V8 SI engine. The inputs to the NN included engine speed, normalized cylinder air charge, spark advance, engine coolant temperature, integrated cylinder air mass since start, relative throttle position, automatic transmission speed ratio, and a metric calculated from crankshaft speed fluctuations generated by modulating the fuel injection pulse width. The output node was linear and standard back-propagation was used to train the NN offline. The performance of the $\mathrm{NN}$ estimator was reported to be slightly better than a ten-parameter regression model used in an earlier study.

Wu and Lee (1998) implemented a two layer feed forward NN with 50 neurons in the hidden layer to model the inverse dynamics of firing event signal dependence on crankshaft speed fluctuation. NN inputs consisted of the fluctuation in crankshaft speed, average values of engine speed and manifold absolute pressure and a sliding window of past input values and output firing 
event signals. The engine was extensively exercised over its operation range (speed and load) for normal firing and four misfire patterns, recording a total of $10^{5}$ firing events. The NN was trained off-line using back-propagation learning rule with an adaptive learning rate. They reported high misfire detection accuracy with a $98.5 \%$ overall probability of detection.

Ortmann et al. (1998) used a feed forward NN architecture with single and double hidden layers to estimate an engine knock index using measured in-cylinder gas pressure. A fast Fourier transform of the pressure signal was used to extract an eight-element feature vector. The NN inputs consisted of the mean, variance, and third moment of the elements of the feature vector over 20 to 100 cycles. Constructive and destructive supervised learning algorithms were used to obtain an optimized NN architecture automatically. Good estimation of the tendency to knock with advanced spark timing was reported, at two engine speeds (2000 and $4000 \mathrm{rpm}$ ).

Ramli and Morris (1993) implemented a two hidden layer (10 neurons each) single-input singleoutput NN to approximate the non-linear characteristics of a fuel injector. Standard backpropagation was used and good approximation accuracy of the non-linear map was reported.

In summary, neural networks are becoming very popular among the IC engine research and development community. Their application, thus far, has focused on the dynamic identification of the air charging process in the intake manifold for either throttle-manifold or turbochargerintercooler-manifold combinations. Static non-linear mapping is also an area where neural nets have been applied extensively for example in the prediction of volumetric and thermal efficiencies, turbocharger characteristics, etc. Very limited implementation to modeling exhaust gas emissions has been reported, though its promise is substantial. Virtually no implementation of neural networks as controllers has been reported which is probably an implementation problem related to 
the computational effort required and the non-proven stability for the case of multi-layer perceptron networks. 


\subsubsection{Control of HD CI Engines on Transient Dynamometers}

Transient testing of IC engines is a progressively important part of the engine development process, particularly medium and heavy-duty compression ignition engines which are mostly open-loop controlled requiring elaborate engine calibration to be conducted on a transient engine dynamometer. The main goal of the test bed controller is to track a pre-specified speed and torque schedule. DC dynamometers, which are capable of absorbing as well as motoring modes, are commonly equipped with a control system that provides integral speed or torque control. Speed control is best accomplished using the dynamometer's controller provided it is adequately fast for speed transients in the test cycle (Clark et al., 1984). Torque control is accomplished by actuating the speed demand lever (accelerator position signal in the case of a drive-by-wire control). Ideally this is accomplished by direct control of the fueling by-passing the governor whether it is a hardware-based (mechanical) or software-based (look up table stored in the ECM) system. For the case of an all-speed governor, the engine fueling is a highly non-linear function of the speed demand. Another aspect is the turbocharger transient response, which is more pronounced for turbocharged engines implementing smoke-limiting fueling strategies under hard acceleration. The strong coupling of the speed and torque control loops makes the definition of response to set point changes and interference impossible (Voos, 1992). These effects as well as the non-linear, multivariable, and time variant interactions of the engine and the dynamometer dynamics complicate the control task. An important consideration in controller design is the time required to achieve a validated test cycle after the installation of the engine. This can only be minimized if the implemented control algorithm is independent of the test schedule. Odaka et al. reported a reduction in the time required to achieve test cycle validation from two hours for an expert using 
a conventional controller to twelve minutes for a predictive control algorithm (Odaka et al., 1990).

Brown and Thompson (1983) achieved independent control of speed and torque by means of a pre-compensation controller, which effectively de-coupled the two control loops. The two singleinput single-output feedback loops employed proportional plus integral controllers. For a 1.1 liter SI engine coupled to an eddy current dynamometer, acceptable control was demonstrated for an independent step-change in speed and torque demand.

Conventional three-term (PID) controllers have proven acceptable for most steady state engine testing requirements. However, their performance is considered inferior compared with the potential of more advanced theories for the case of transient testing (Noble et al., 1988; Odaka et al., 1990). Koustas and Watson (1984) implemented a torque control algorithm based on time optimal control via an eddy-current dynamometer excitation model and a speed control based on a PID superimposed on a time optimal control via a dynamic model of the actuation stepper motor and load system. Pre-view of the prescribed test cycle was used to improve tracking performance. Koustas and Watson reported that repeatability of the system performance was below 5\% standard deviation on torque and speed, and good tracking accuracy for a highly transient portion of the FTP test cycle was obtained.

Noble et al. (1988) reported tight control of transient speed and torque when using system identification coupled with a generalized predictive control algorithm to design an optimal controller with a finite-receding horizon on a test bed equipped with a standard eddy current dynamometer. The test bed was modeled as a two-input (throttle position and dynamometer excitation current), two-output system (engine speed and dynamometer torque). The speed and 
torque responses were then regarded as separate two-input, one-output processes represented by controlled auto-regressive integrated moving average models.

Tuken et al. (1990) implemented a predictive torque control algorithm for a turbocharged diesel engine coupled to a DC dynamometer with a speed controller. Off-line parameter identification resulted in a second order transfer function plus a time delay model. Closed-loop pole assignment was used to determine the parameters of a lag-lead torque controller. Good repeatability and tracking performance were reported over the FTP test cycle. Gain scheduling was proposed to eliminate offsets between actual torque values and reference torque trajectories due to parameter variations of the plant and disturbances transfer functions over different operating ranges.

Odaka et al. (1990) developed a two step predictive speed control algorithm for a DI HD diesel engine connected to a DC dynamometer. Prior to control, an engine performance model was developed through exercising the engine under steady state operation where the dynamometer controls engine speed and torque control was achieved through throttle actuation. In the automatic operation, the developed model was used to set the position of the throttle based on the prescribed speed and feedback control was used to compensate for minor deviations. The dynamometer excitation unit was used to set the engine torque using feedback control. Improved test repeatability, tracking accuracy, and stability of exhaust gas emissions were reported compared to a conventional PID controller over the FTP test cycle. Odaka et al. reported significant reduction in carbon monoxide emissions and smoke during hard acceleration when implementing the predictive speed controller.

Miller (1995) implemented a combination of on-off 'bang-bang' and proportional feedback actuation of the throttle position to track the FTP torque schedule for several CI turbocharged engines on a test bed equipped with a DC dynamometer. The method used the 'bang-bang' 
control only for large rising torque transients (torque transients greater than $40 \%$ of the maximum torque at a given speed). The throttle position was held at $100 \%$ until the torque set point was reached, then the throttle position was reset to a value determined by a look up table (throttle position as a function of engine speed and torque derived from steady state sweeps) and modulated by feedback control. Miller reported improvement in regression results for torque for engines with significant turbocharger lags when compared to a simple proportional feedback control. A persistent increase in NOx emissions $(\sim 1 \%-8 \%)$ was reported due to the more aggressive control.

In view of the above review, using a neural network-based approach to control engine torque over the test cycle is very attractive because of the inherent non-linearity and adaptive requirement of the control task. However, it must be acknowledged that the lack of theoretically proven stability criteria for multi-layer perceptron neural network-based controllers complicates the certification procedure. The synthesis of the proposed controller is greatly facilitated in a simulation environment using an engine model before actual implementation. Further, the coupling of the engine model with an emissions prediction model becomes essential in view of the impact of the adopted control algorithm on transient emissions. 


\section{CHAPTER 2}

\section{Experimental Procedure}

The purpose of this section is to report details of the engine used in the development of the modeling and control described in this thesis, the emissions measurement analyzers, the data acquisition hardware and developed software, the data reduction procedures and developed software, and the test cycles used.

\subsection{Engine}

A Navistar T444E diesel engine was used in this study. It is a four-stroke turbocharged V-8 with overhead valves with a displacement of 444 cubic inches (7.3 liters). It has a compression ratio of $17: 1$ and is rated at $190 \mathrm{bhp}$ at $2600 \mathrm{rpm}$ and $485 \mathrm{ft}-\mathrm{lbf}$ at $1500 \mathrm{rpm}$. The T444E fuel system provides filtered fuel to the injectors at a regulated $40 \mathrm{psi}(276 \mathrm{kPa})$. A distinctive feature of the T444E is the use of engine oil to actuate the fuel injectors hydraulically with electronically controlled opening of the injector valve (the HEUI system). When the injector solenoid is energized, oil is allowed to flow into the injector and to act on an intensifier piston that amplifies the pressure of the injected fuel by a 7:1 ratio. Pressure control in the injection control pressure system is closed-loop. An electronically controlled regulator valve achieves injection control pressure regulation by dumping excess oil back to the sump. The injection control pressure ranges from 500 to 3000 psi (Hower et al., 1993). The stock EEC-IV engine management module is capable of providing control over injection timing, injected fuel quantity, and injection pressure. British Petroleum \#2 diesel fuel was used and its temperature was pre-conditioned to an average temperature of $87^{\circ} \mathrm{F}$.

A direct-current General Electric dynamometer was used to motor and load the engine. A load cell measured the static engine brake torque through a lever arm mounted on the dynamometer 
housing. Closed-loop control of the engine speed was achieved using a GE dynamometer controller. The engine brake torque was closed-loop controlled using proportional integral actuation of the fueling input, employing a voltage signal to the engine control module in lieu of the accelerator pedal position.

\subsection{Emissions Measurement}

As per Code of Federal Regulations (40CFR Part 86 Subpart N, 1996), the exhaust gas was routed to a full scale dilution tunnel where the exhaust gas was diluted with pre-conditioned air at $68^{\circ} \mathrm{F}$ and $70 \%$ relative humidity. The exhaust gas and the dilution air was pulled by a blower through a metering venturi operating at a choked condition; the venturi can be changed for different engine (flow) sizes. The emissions concentrations of total hydrocarbon (THC), carbon dioxide $\left(\mathrm{CO}_{2}\right)$, carbon monoxide $(\mathrm{CO})$, oxides of nitrogen $(\mathrm{NOx})$, and particulate matter $(\mathrm{PM})$ were measured continuously. The THC concentration was measured with a flame ionization detector (Rosemount Analytical Model 402) and reported as parts-per-million carbon. The $\mathrm{CO}_{2}$ and $\mathrm{CO}$ concentrations were measured with non-dispersive infrared analyzers (Rosemount Analytical Model 880A). A chemiluminescence analyzer (Rosemount Analytical Model 955 NO/NOx Analyzer) was used for the detection of NOx emissions as parts-per-million nitric oxide. The particulate mass concentration was quantified in real time using a tapered element oscillating microbalance device (Rupprecht \& Patashnick Co. TEOM Model 1105). 


\subsection{Data Acquisition}

The data acquisition system incorporated a Keithley Metrabyte CTM10 counter/timer module and a DAS16 data acquisition board. The CTM10 was used to measure time or frequency-related parameters. A Rotunda breakout box was used to allow for the simultaneous monitoring of the engine control module (ECM) signals such as the fuel injection pulse width, injection timing, and injection control pressure. The DAS16 was used to measure analog signals. An acquisition program was developed in Quick Basic, by the author, which allows for flexible setting of the sampling rate as well as channel assignments. Control of the engine and data acquisition was performed via computers in a room adjacent to the engine dynamometer. Details of enginedynamometer setup, channel assignment, data handling procedure, and program listing are provided in the reference by Atkinson et al. (1998b).

\subsection{Data Reduction}

A reduction program was developed in $\mathrm{C}$ language, by the author, which processes the acquired data files and performs a multitude of tasks in a batch mode. It reads two list data files of runs and channel assignments, respectively. The channel data file includes the attributes of the measured and calculated parameters. The measured ADC codes are converted to engineering units. The program provides provision for data filtering including weeding out unbounded values and smoothing (low pass filtering to eliminate high frequency noise). The engine operating variables are calculated and the measured emissions concentration are shifted in time to account for the time delays due to the finite response time of each analyzer and the transport delay in the dilution tunnel and the sampling lines. The reduction program is capable of generating integral results over the test cycle, calculating the time shift to maximize cross correlation of the nth derivative of any two variables, generating look up maps of any (m) variables in (n) independent 
variables, and variable estimation using previously generated maps. A listing of the reduction program is available through the author.

\subsection{Test Cycles}

The engine was tested over the Federal Test Procedure (FTP) as well as over a set of eight randomly generated transient cycles. The purpose of using the arbitrary cycles was to exercise the engine extensively over its full operating range. For all of the tests, the stock controller calibration was retained and the engine was maintained at a normal operating temperature.

Eight arbitrary cycles were developed from a random cycle generator based on the "walking man" problem where a random path in the speed and load domain was followed. A matrix of speed and load points was used to develop each cycle. The minimum speed, maximum speed, minimum torque, and maximum torque of the engine are specified as inputs. The maximum torque can be set to a constant value or it can be obtained from the maximum 'mapped' torque curve of the engine. The speed and load matrix is divided into an $\mathrm{M}$ by $\mathrm{N}$ matrix where the dimensions $\mathrm{M}$ and $\mathrm{N}$ are set to minimize the transient from one speed and load set point to the next point. The cycle visits each point in the $\mathrm{M}$ by $\mathrm{N}$ matrix in a quasi-random fashion. At each arbitrary point in the matrix, eight possible next locations can be selected as shown in Figure 2.1. For a point $X$ in the matrix, the speed can change with a subtle change in the torque (point 4 or 5), the load can change with constant speed (point 2 or 7), or the speed and load can change

concurrently (point 1, 3, 6, or 8). The speed spacing is constant between the minimum and maximum values. However, if the maximum torque is set to the maximum load curve, then the torque spacing varies for a given speed point, as illustrated in Figure 2.1.

To minimize the number of times each given point is visited, a counter is used to weight the visited point. When determining which point to visit next in the cycle, the next possible point 
with the minimum weight is selected. For points with an equal minimum weight, an arbitrary direction is selected within the program. To minimize the time spent in the negative torque or motored region, an initial weight can be given to some or all of the negative torque points to minimize the number of times these points are visited. The number of points in the speed-load matrix and the time spent at each point can also be varied.

The speed and load are specified in one-second intervals. The time spent at each speed-load point can vary from one second to an unlimited maximum value, which permits the development of cycles that range from being highly transient to step tests. The time step can also vary from point-to-point and is specified as an average value with a range in which it can vary, $t \pm \Delta t$. Table 2.1 provides a description of the parameters specified for generating the eight arbitrary cycle used in this study. The average time spent at each point for cycles A and B is 10 seconds while the average time spent at each point in the other six cycles is one or two seconds. The cycles $\mathrm{C}$ to $\mathrm{H}$ are more aggressive than $\mathrm{A}$ and $\mathrm{B}$, and several of these are more aggressive than the FTP.

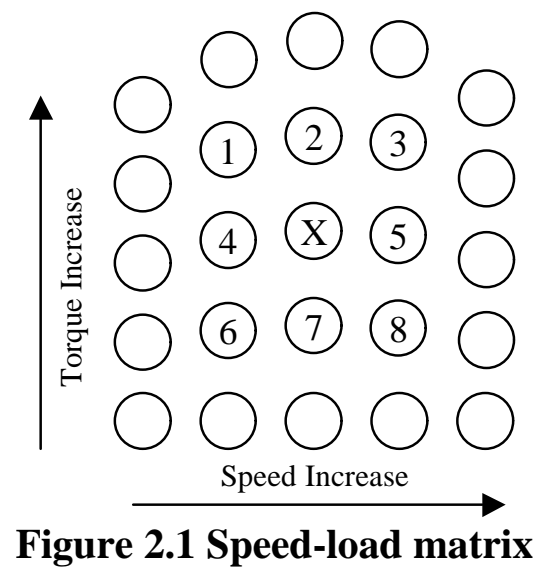

The speed and torque time trace and the corresponding speed-torque matrix for the FTP and cycles A (gentle) and E (aggressive) are shown in Figures 2.2 and 2.3. The FTP cycle spends the majority of time above an engine speed of $1800 \mathrm{rpm}$. Meanwhile, the eight randomly generated cycles do not show any such bias towards any set point. 


\subsection{Emissions Signal Reconstruction}

The measured exhaust emissions data were shifted to account for the time delays due to the finite response time of each analyzer and the transport delay in the dilution tunnel and sampling system. A cross correlation (CC) scheme, based on the inner product of the normalized signals, was developed to ensure a logical action-reaction time sequence.

The influence of engine speed on the maximum correlation time shift was examined by pulsing the accelerator position signal (APS) while holding the engine speed over a range of speeds from 800 to $2200 \mathrm{rpm}$ with a step size of $200 \mathrm{rpm}$. The APS pulse had a period of five seconds at a frequency of $0.04 \mathrm{~Hz}$ and amplitude of $25 \%$. Time shifts to maximize the $\mathrm{CC}$ of the zeroth and first derivatives of the examined parameters were conducted.

Cross correlation of brake mean effective pressure (BMEP) with carbon dioxide $\left(\mathrm{CO}_{2}\right)$ and oxides of nitrogen (NOx) at different time shifts are shown in Figure 2.4 and Figure 2.5, respectively.

Table 2.1 Test cycle parameters

\begin{tabular}{|l|c|c|c|c|c|c|c|c|c|}
\hline \multirow{3}{*}{ Cycle } & \multicolumn{3}{|c|}{ Speed } & \multicolumn{3}{c|}{ Torque } & \multicolumn{3}{c|}{ Time } \\
\cline { 2 - 11 } & Min & Max & Steps & Min & Max & Steps & Points & Average & $\pm \Delta t$ \\
\cline { 2 - 11 } & Rpm & rpm & - & ft-lb & ft-lb & - & - & sec & sec \\
\hline FTP & 667 & 2525 & NA & -177 & 421 & NA & 1199 & NA & NA \\
\hline ARB A & 700 & 2650 & 5 & -100 & 445 & 5 & 100 & 10 & 3 \\
\hline ARB B & 700 & 2650 & 5 & -49 & 445 & 5 & 100 & 10 & 1 \\
\hline ARB C & 700 & 2650 & 8 & -49 & 442 & 6 & 300 & 2 & 1 \\
\hline ARB D & 700 & 2650 & 6 & 0 & 442 & 6 & 300 & 1 & 0 \\
\hline ARB E & 700 & 2650 & 10 & 0 & 445 & 10 & 600 & 1 & 0 \\
\hline ARB F & 700 & 2650 & 6 & 0 & 442 & 6 & 400 & 2 & 1 \\
\hline ARB G & 700 & 2650 & 6 & 0 & 442 & 6 & 500 & 1 & 0 \\
\hline ARB H & 700 & 2650 & 5 & -49 & 445 & 6 & 500 & 2 & 1 \\
\hline
\end{tabular}


Results are shown for selected transient test cycles as well as the pulsed APS experiments. The time shifts for maximum CC vary by 1 to 2 seconds over the examined cycles. Also, the peak is much more defined for the first derivative cross correlation when compared to the zeroth derivative values, especially over the transient test cycles.

Figure 2.6 shows the $\mathrm{CC}$ results for $\mathrm{CO}_{2}$ with $\mathrm{LCO}$ at different time shifts. The $\mathrm{CO}_{2}$ signal was used as the reference signal to account for the transport time delay of the $\mathrm{CO}$ signal because they were sampled using the same probe, dehumidified through a refrigeration unit, and quantified using two identical analyzers. The $\mathrm{CO}$ signal lags a few seconds behind the $\mathrm{CO}_{2}$ signal over all the examined test cycles. Again, the CC peak is much more pronounced for the first derivative cross correlation, as shown in Figure 2.6.

The transport delay associated with the THC signal is more difficult to determine since its mechanism of formation is much more complex with multiple dependencies. CC results with LCO at different time shifts are shown in Figure 2.7. For first derivative CCs, a peak is observed at a time shift of -10 seconds, corresponding to a transport delay of 6 seconds for 16 seconds delay in the $\mathrm{CO}$ signal, which is more pronounced at higher engine speed and higher transient operation.

Figure 2.8 shows the CC results for PM signal with commanded fuel rate at different time shifts. Results are shown for selected transient test cycles. PM measurement for the APS experiments was not conducted. Consistent peaks of the first derivative CCs were observed at a delay period of 9 seconds over the examined transient cycles.

The time shifts for maximum CC of first derivatives are shown in Table 2.2a for the APS experiments and Table $2.2 \mathrm{~b}$ for the transient test cycles. The repeatability of the CC approach was guaranteed independently of the test schedule, especially for the emissions of $\mathrm{CO}_{2}, \mathrm{NOx}$, 
and PM. The maximum CC shift time algorithm enforced a nominal shift when it failed to locate a pronounced peak. This explains the good repeatability in the calculated THC time shift. It is concluded that the maximum cross correlation of first derivatives is an acceptable scheme to determine the time delays for the measured emissions signals.

Table 2.2a Time shifts in seconds for maximum $\mathrm{CC}$ for pulsed throttle tests

\begin{tabular}{|c|c|c|c|c|c|c|c|c|}
\hline \multicolumn{5}{|c|}{ TEST CYCLE } & \multirow{2}{*}{$\frac{\mathrm{THC}}{6.0}$} & \multirow{2}{*}{$\frac{\mathrm{LCO}}{16.1}$} & \multirow{2}{*}{$\frac{\mathrm{CO}_{2}}{16.4}$} & \multirow{2}{*}{$\frac{N O x}{10.6}$} \\
\hline $\mathrm{TP}$ & PLS & $00 \div-25 \%$ & a 0800 & rpm & & & & \\
\hline $\mathrm{TP}$ & PLS & $00 \div-25 \%$ & a 1000 & rpm & 6.0 & 18.5 & 15.2 & 10.7 \\
\hline $\mathrm{TP}$ & PLS & $00 \div-25 \%$ & e 1200 & $\mathrm{rpm}$ & 6.1 & 16.7 & 14.7 & 10.8 \\
\hline $\mathrm{TP}$ & PLS & $00 \div-25 \%$ & a 1400 & rpm & 6.0 & 19.3 & 16.0 & 10.4 \\
\hline $\mathrm{TP}$ & $\mathrm{PLS}$ & $00 \div-25 \%$ & (c) 1600 & rpm & 5.8 & 18.2 & 15.8 & 10.1 \\
\hline & PLS & $25 \%-50 \%$ & e 1800 & rpm & 6.0 & 16.3 & 15.2 & 10.1 \\
\hline & PLS & $30 \div-55 \%$ & e 2000 & rpm & 5.8 & 16.1 & 14.9 & 10.2 \\
\hline & PLS & $35 \%-60 \%$ & e 2200 & rpm & 5.9 & 16.4 & 15.4 & 10.3 \\
\hline & & & & Mean & 6.0 & 17.2 & 15.5 & 10.4 \\
\hline & & & & Stdv & 0.1 & 1.3 & 0.6 & 0.3 \\
\hline & & & & $\mathrm{Cov}$ & $1.80 \%$ & $7.36 \%$ & $3.73 \%$ & $2.62 \%$ \\
\hline
\end{tabular}

Table 2.2b Time shifts in seconds for maximum $\mathrm{CC}$ for transient cycle tests

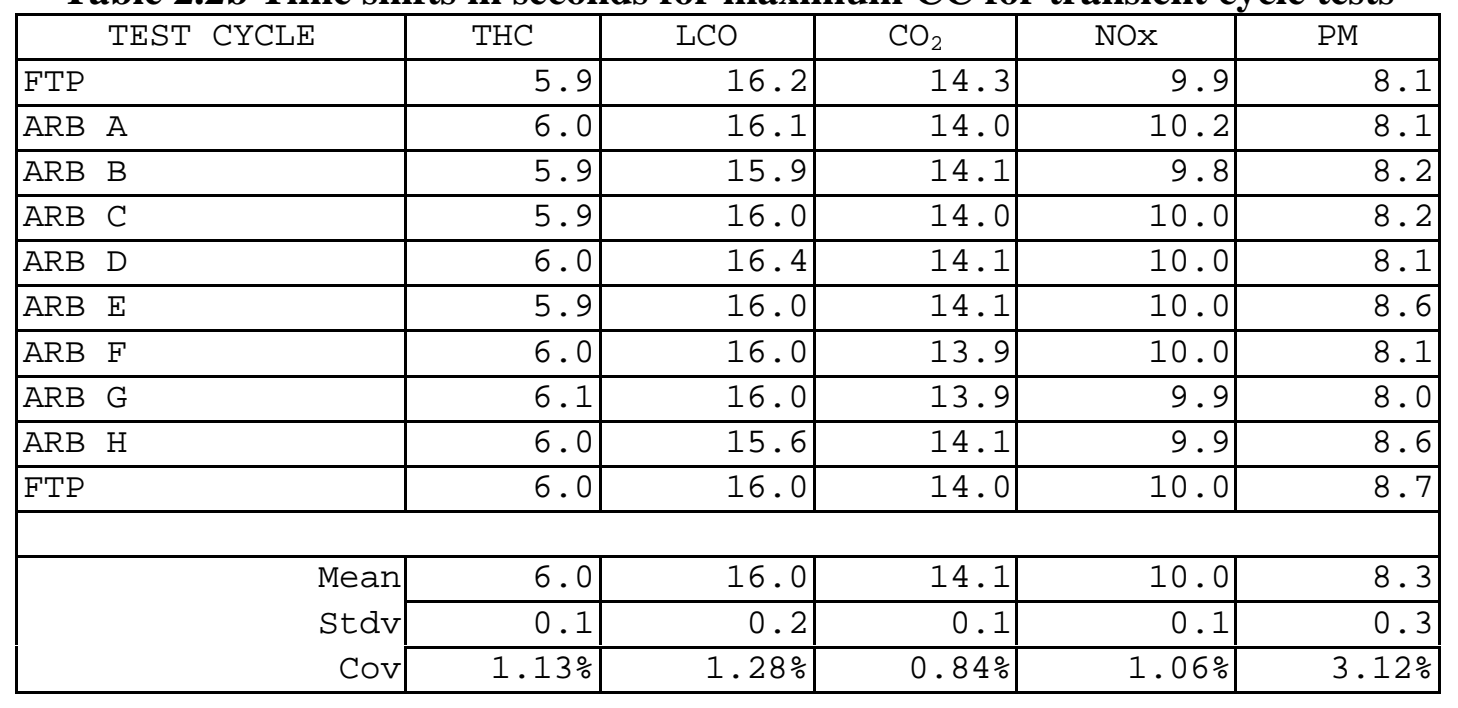

The normalized reconstructed signals are shown in Figure 2.9 for the selected pulsed APS experiments. The axial dispersion of the emissions signals is evident and more pronounced for the CO signal. The problem of identifying the transport delay of the THC signal, especially at low engine speeds, is attributed to the low amplitude of response to sharp transients in fueling. It 
must be noted that no attempt was made to de-convolute the emissions signal to account for the distortions introduced by the sampling system. It is recognized that this would result in some inaccuracies in the instantaneous transient emissions predictions which are not expected to influence the integral result over any given test schedule. Furthermore, including this damping effect in any modeling effort (e.g. incorporating a sliding window, which is large enough to reproduce the mixing effect in the sampling train (including the sampling system in the model) would facilitate the validation process. 


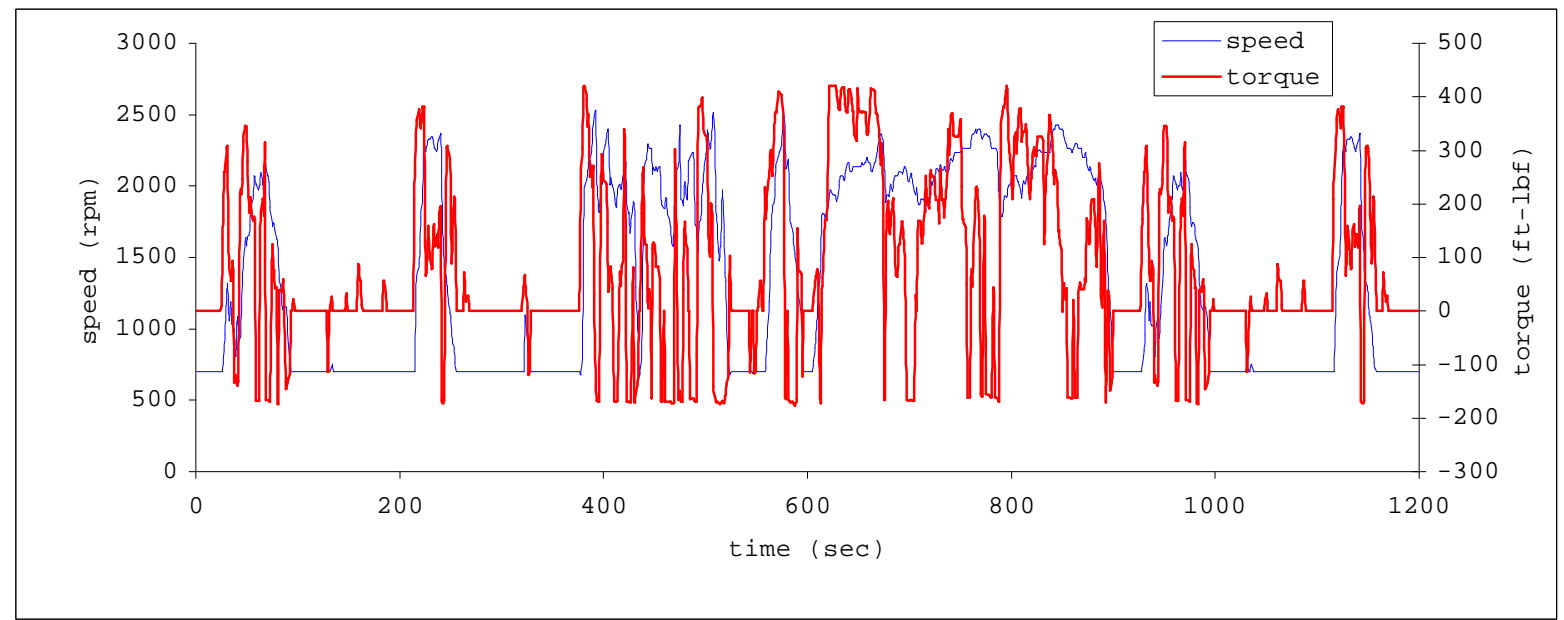

Figure 2.2a FTP speed and torque time trace

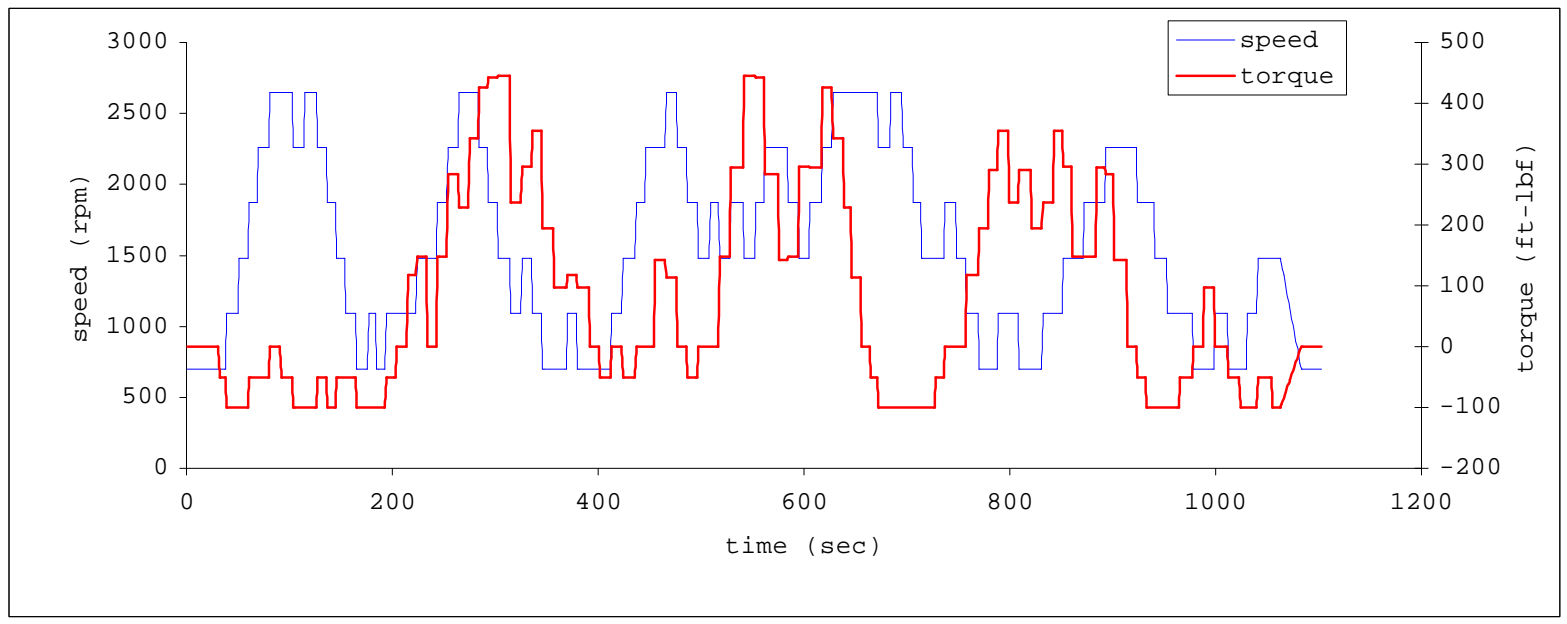

Figure 2.2b Arbitrary Cycle A speed and torque time trace

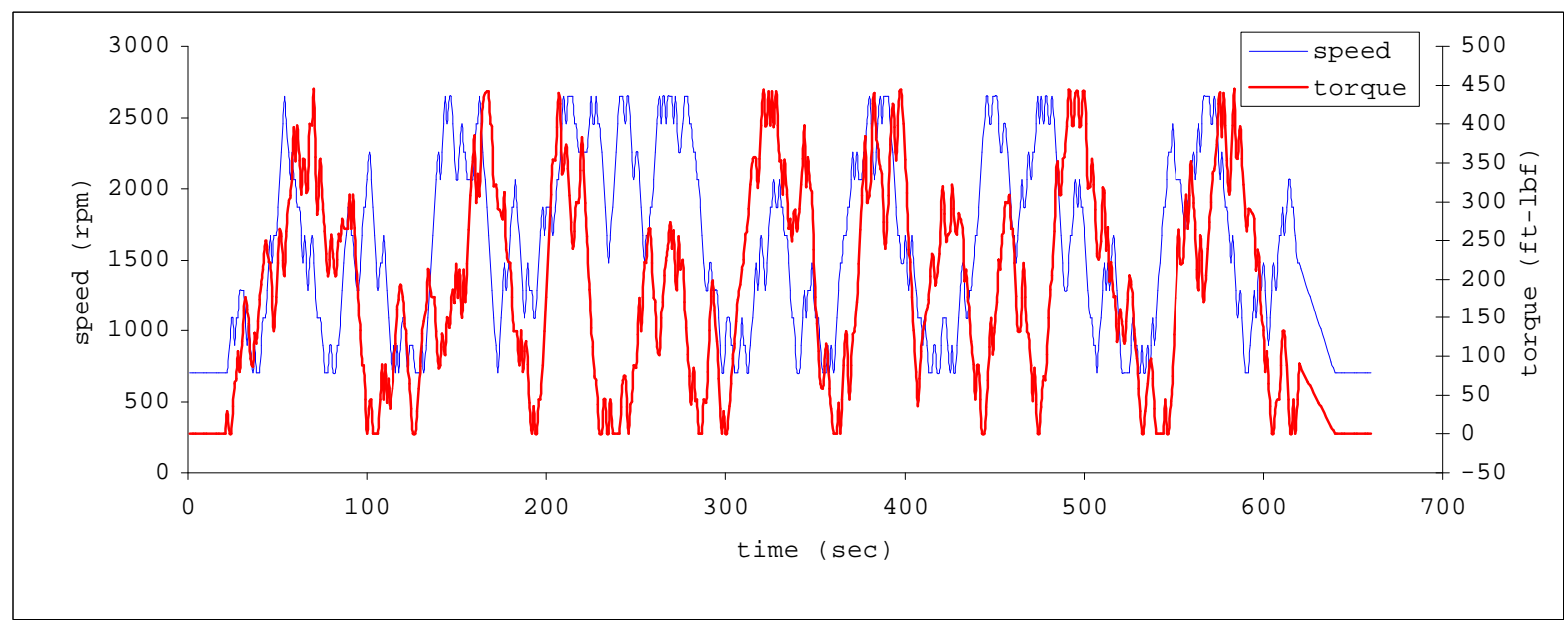

Figure 2.2c Arbitrary Cycle E speed and torque time trace 


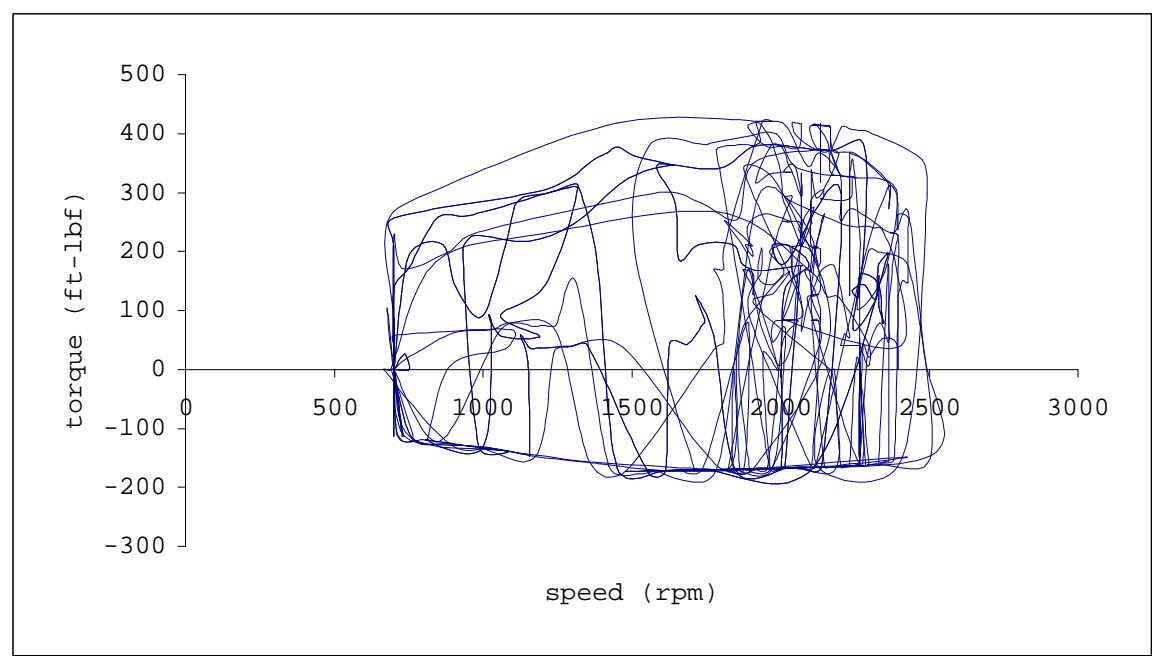

Figure 2.3a FTP speed-torque matrix

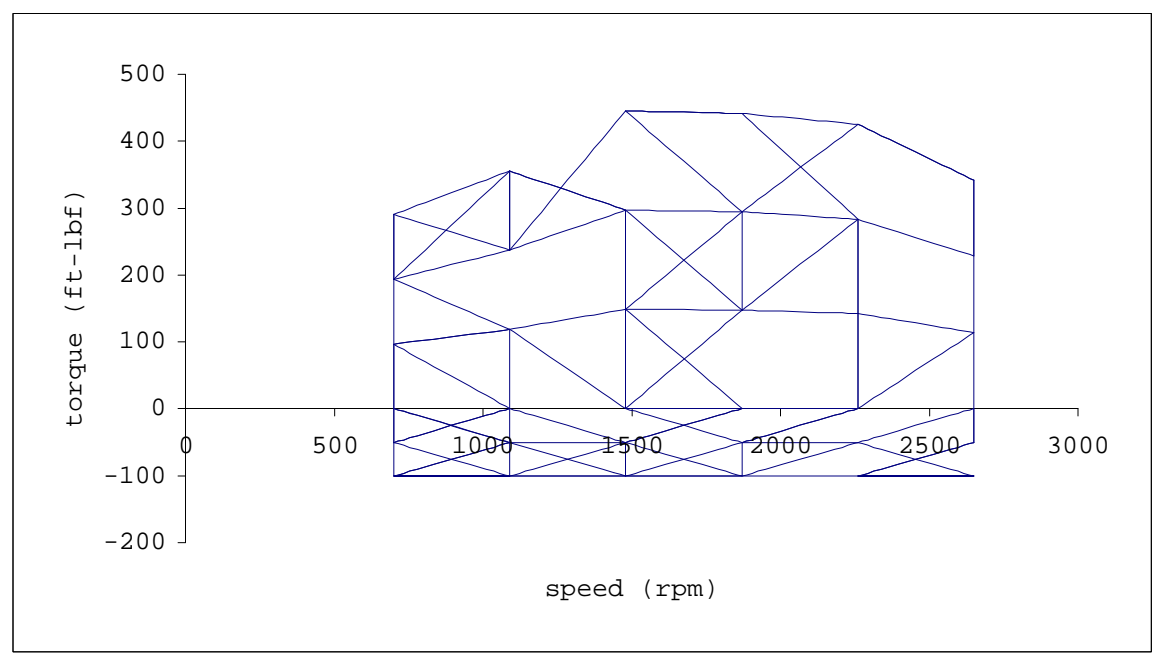

Figure 2.3b Arbitrary Cycle A speed-torque matrix

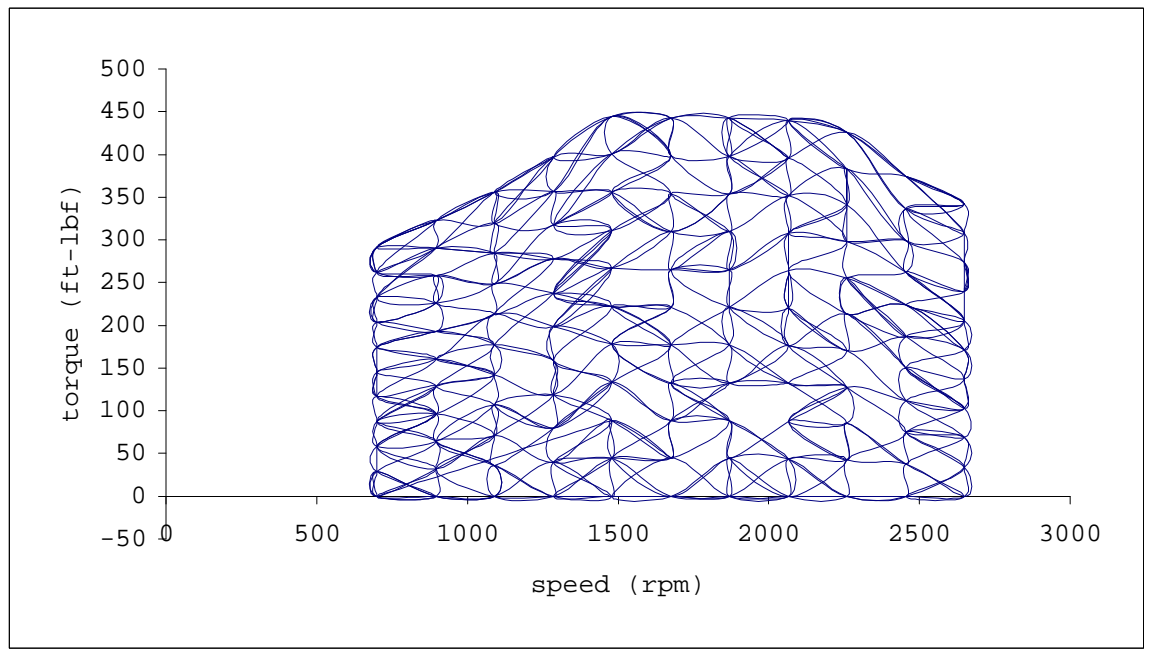

Figure 2.3c Arbitrary Cycle E speed-torque matrix 


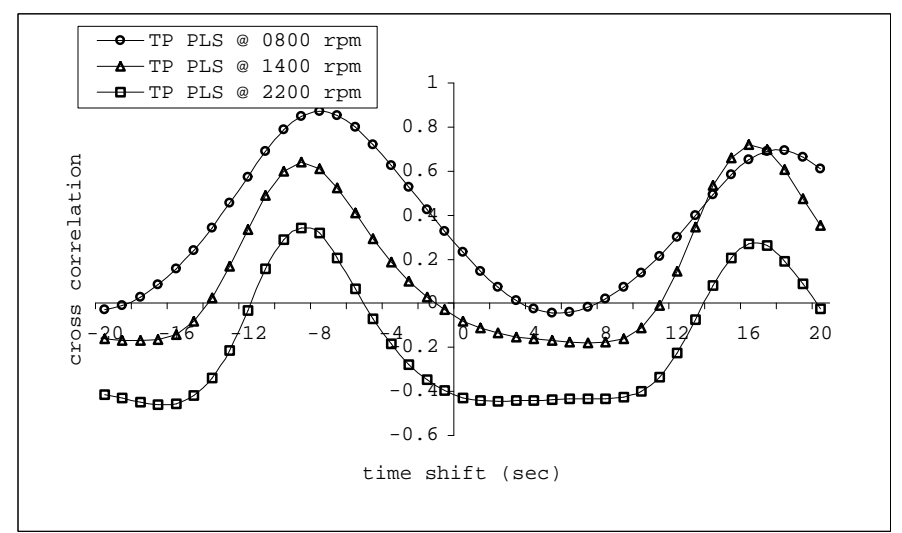

Figure 2.4a BMEP-CO2 zeroth derivative cross correlation for pulsed throttle tests

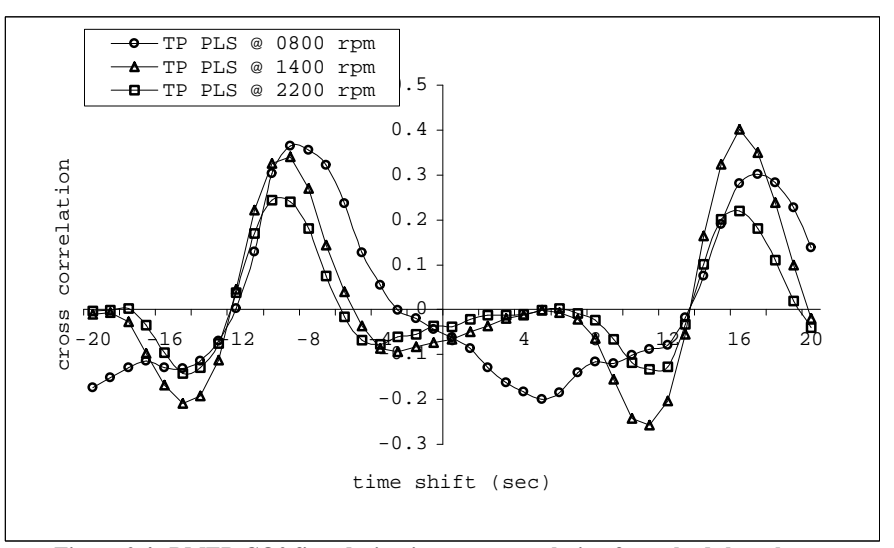

Figure 2.4c BMEP-CO2 first derivative cross correlation for pulsed throttle tests

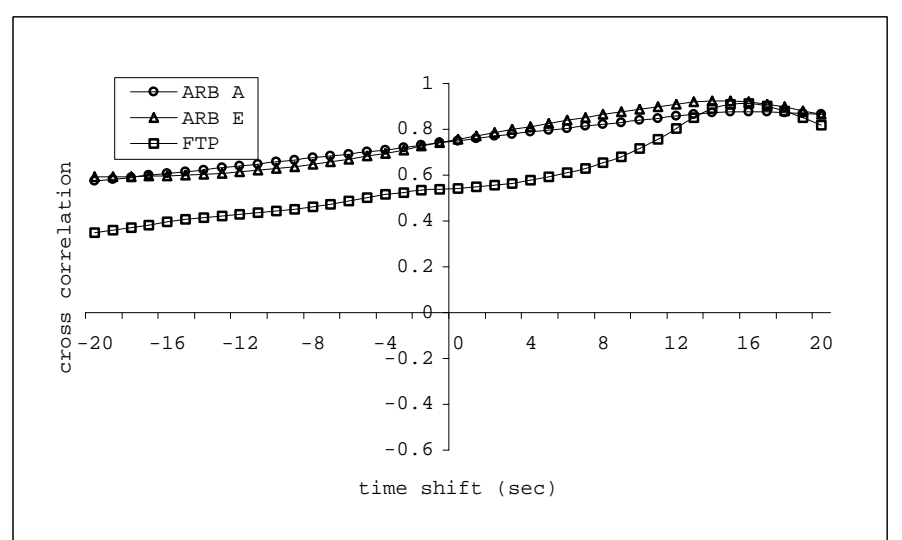

Figure 2.4b BMEP-CO2 zeroth derivative cross correlation for transient tests

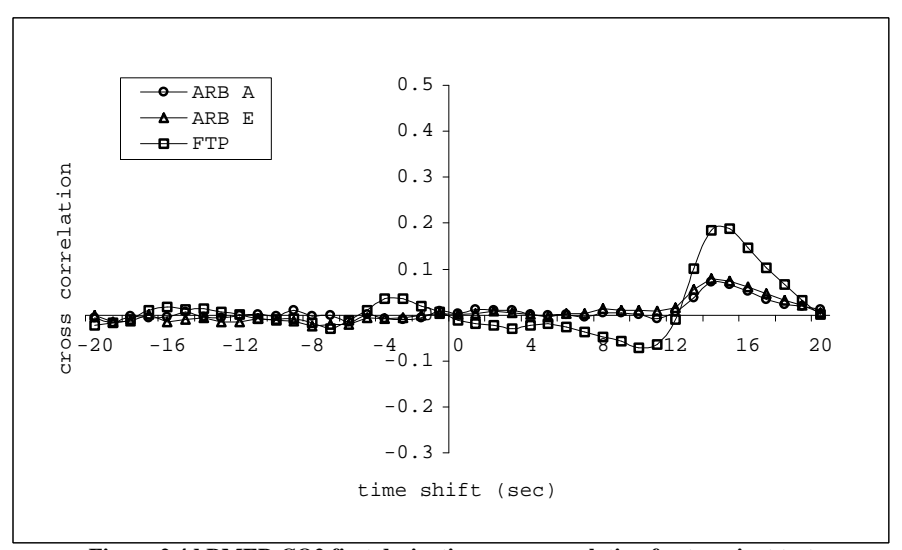

Figure 2.4d BMEP-CO2 first derivative cross correlation for transient tests 


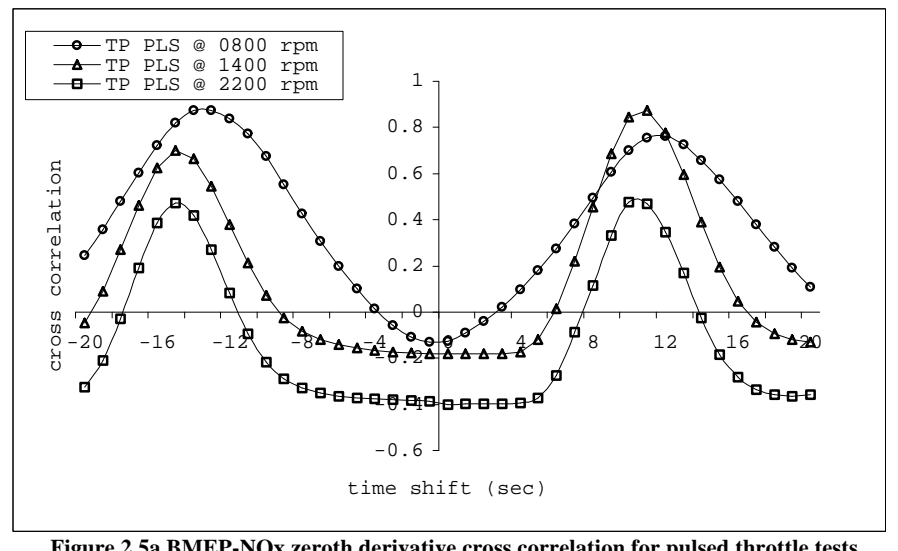

Figure 2.5a BMEP-NOx zeroth derivative cross correlation for pulsed throttle tests

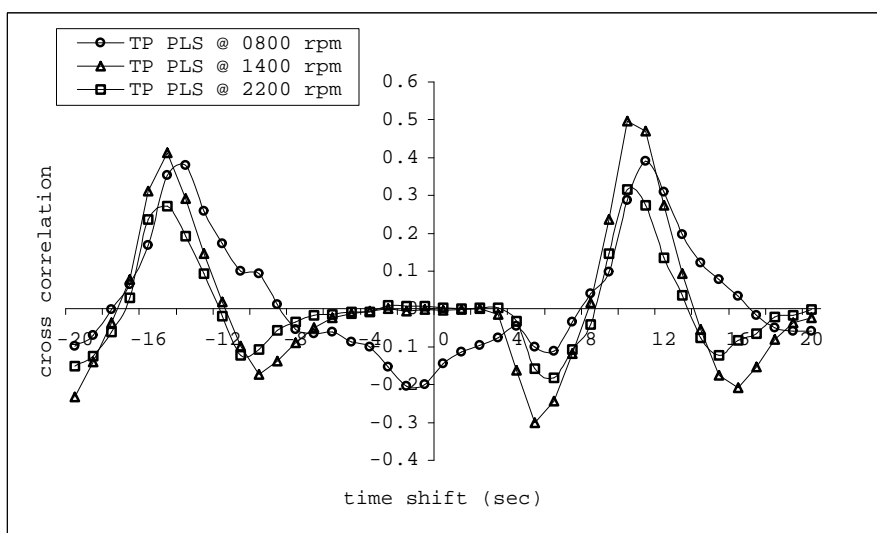

Figure 2.5c BMEP-NOx first derivative cross correlation for pulsed throttle tests

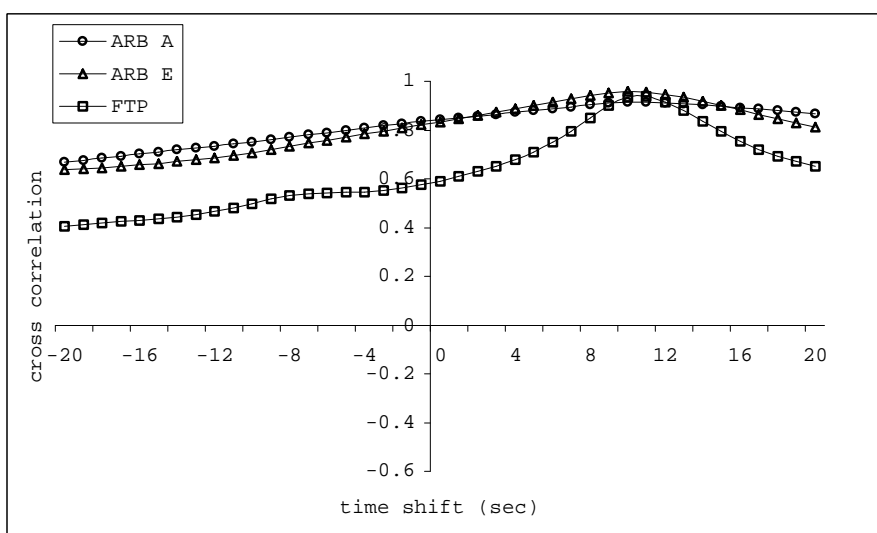

Figure 2.5b BMEP-NOx zeroth derivative cross correlation for transient tests

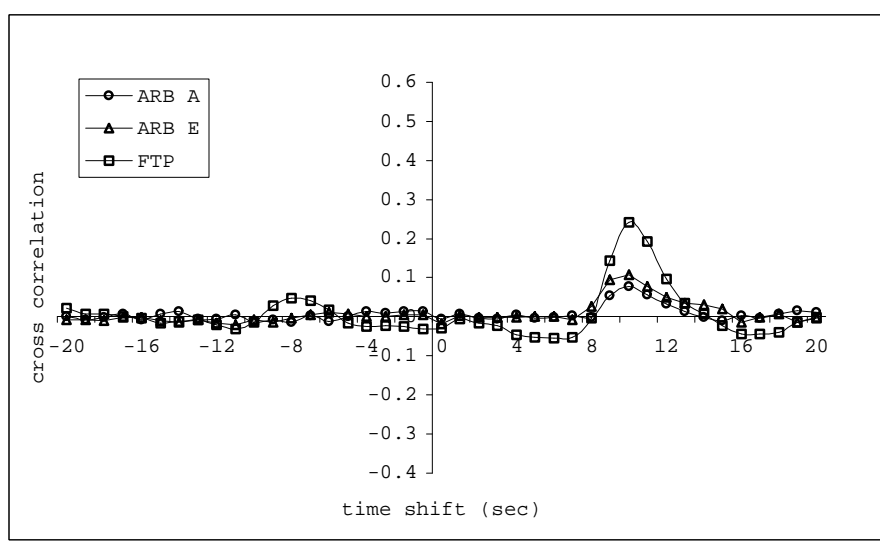

Figure 2.5d BMEP-NOx first derivative cross correlation for transient tests 


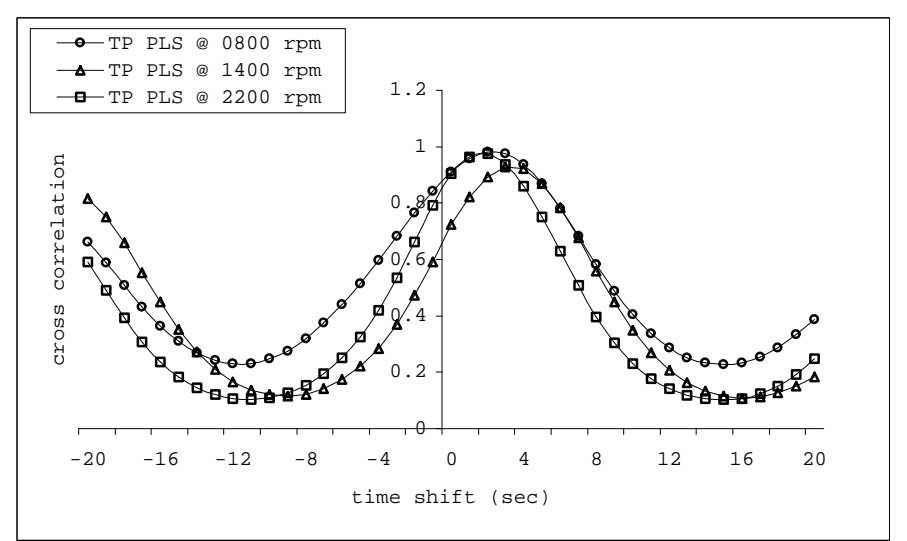

Figure 2.6a CO2-LCO zeroth derivative cross correlation for pulsed throttle tests

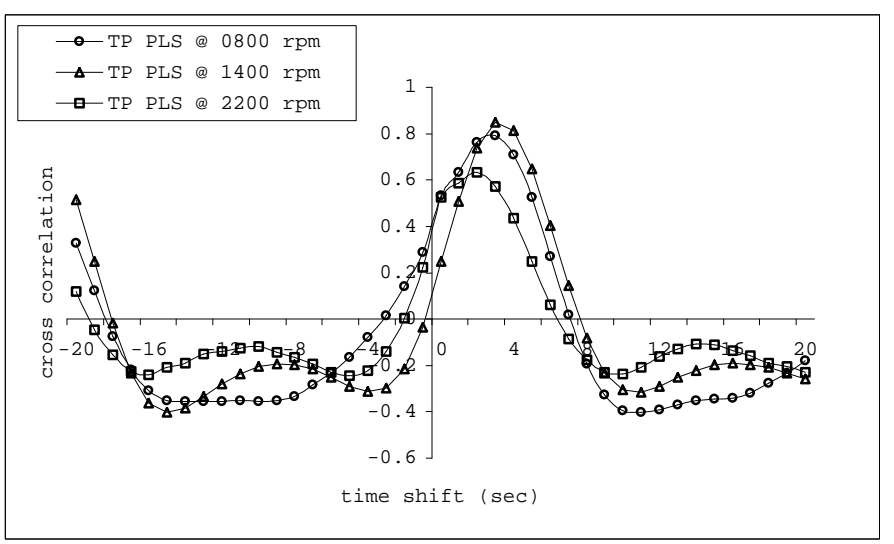

Figure 2.6c CO2-LCO first derivative cross correlation for pulsed throttle tests

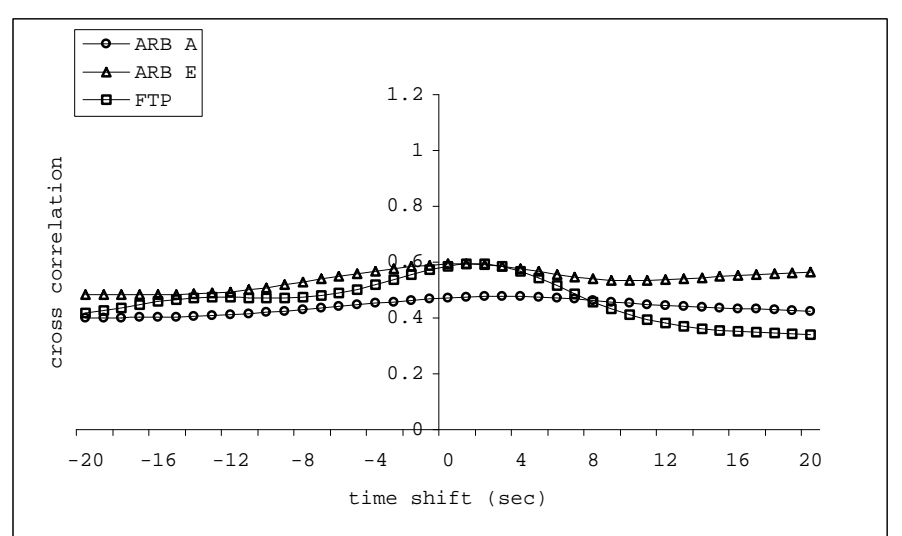

Figure 2.6b CO2-LCO zeroth derivative cross correlation for transient tests

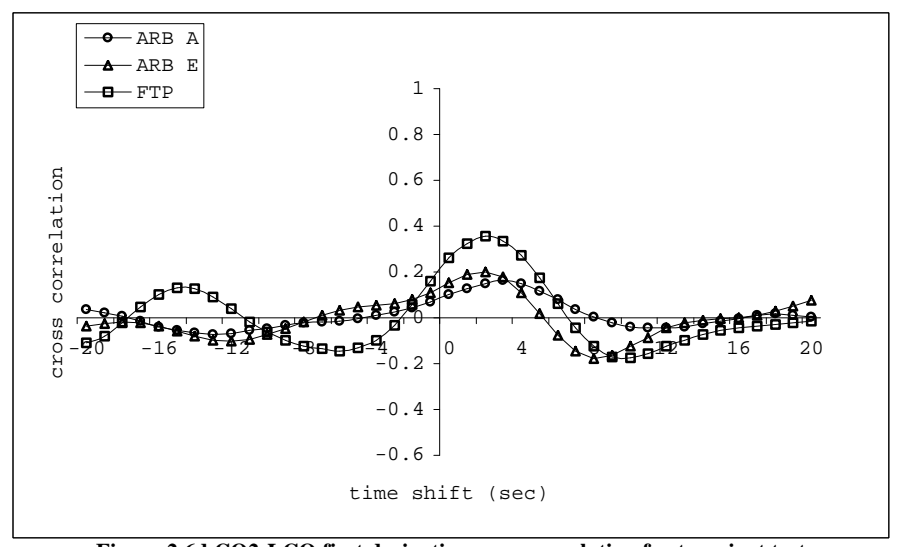

Figure 2.6d CO2-LCO first derivative cross correlation for transient tests 


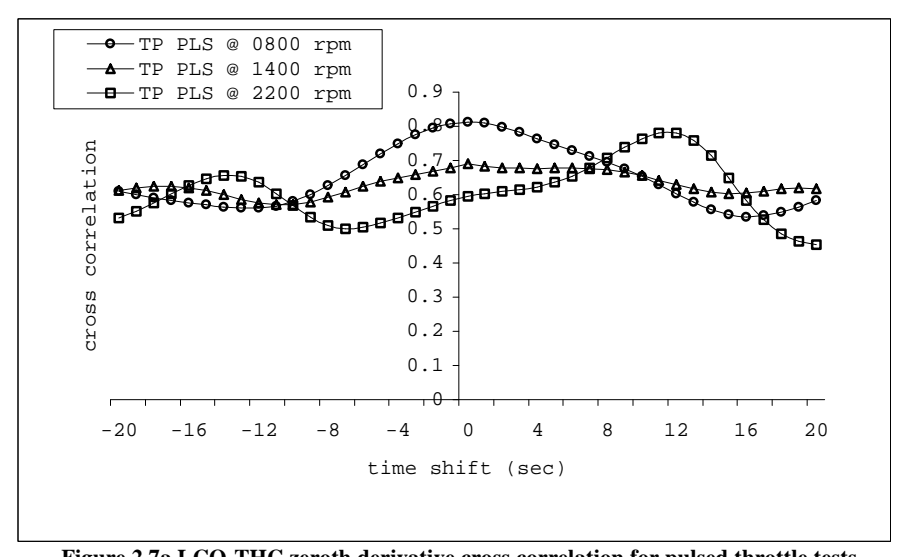

Figure 2.7a LCO-THC zeroth derivative cross correlation for pulsed throttle tests

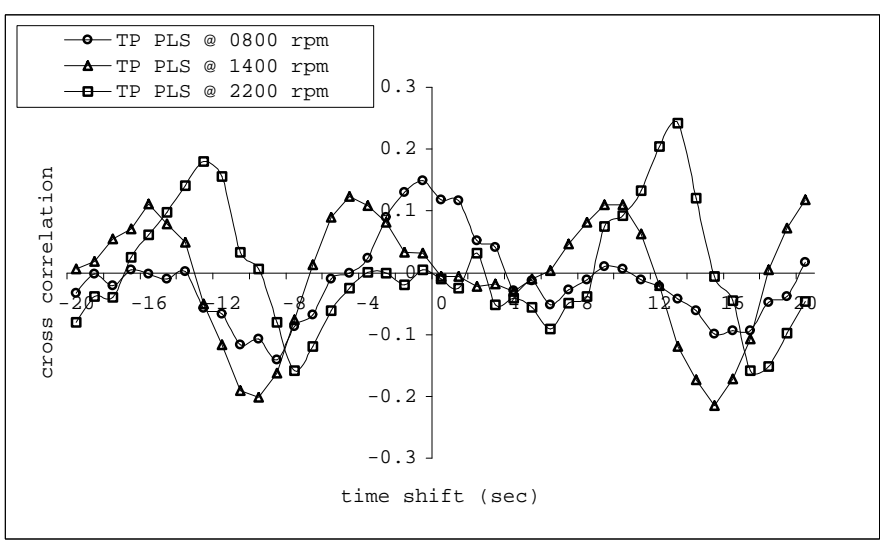

Figure 2.7c LCO-THC first derivative cross correlation for pulsed throttle tests

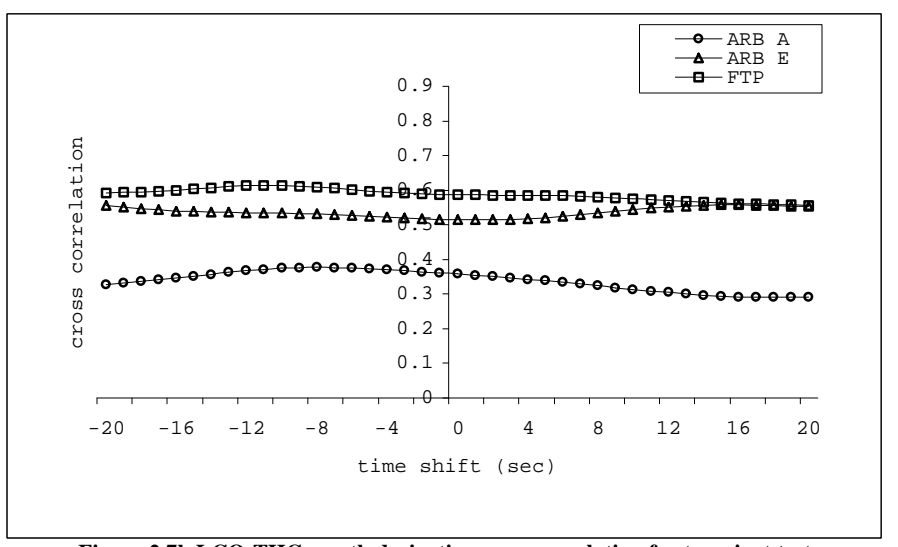

Figure 2.7b LCO-THC zeroth derivative cross correlation for transient tests

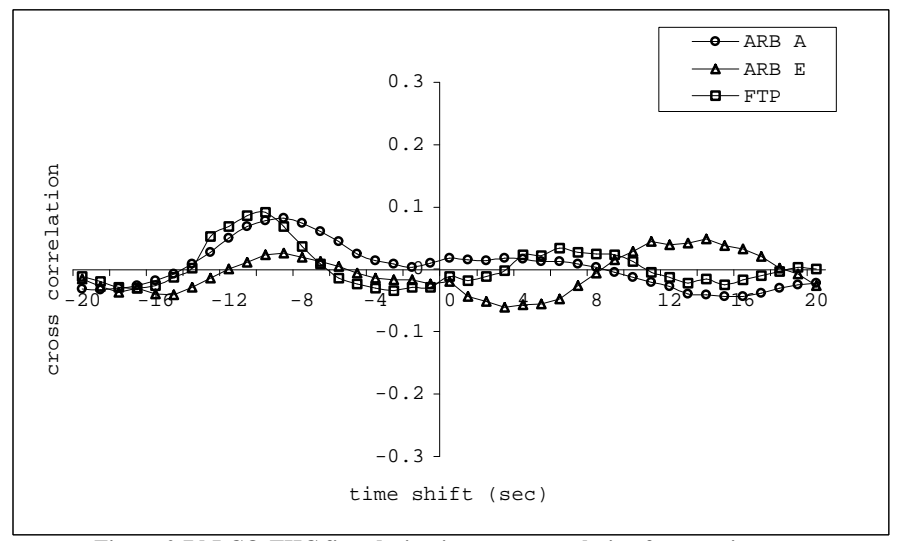

Figure 2.7d LCO-THC first derivative cross correlation for transient tests 


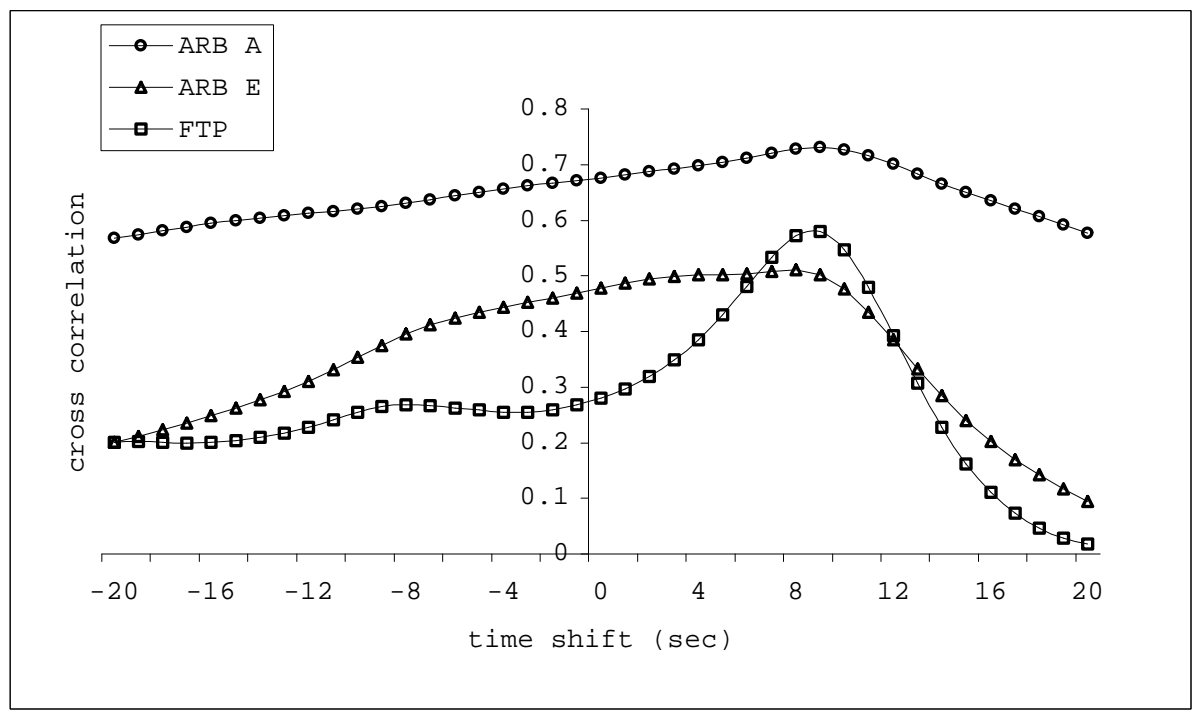

Figure 2.8a FUEL-PM zeroth derivative cross correlation for transient tests

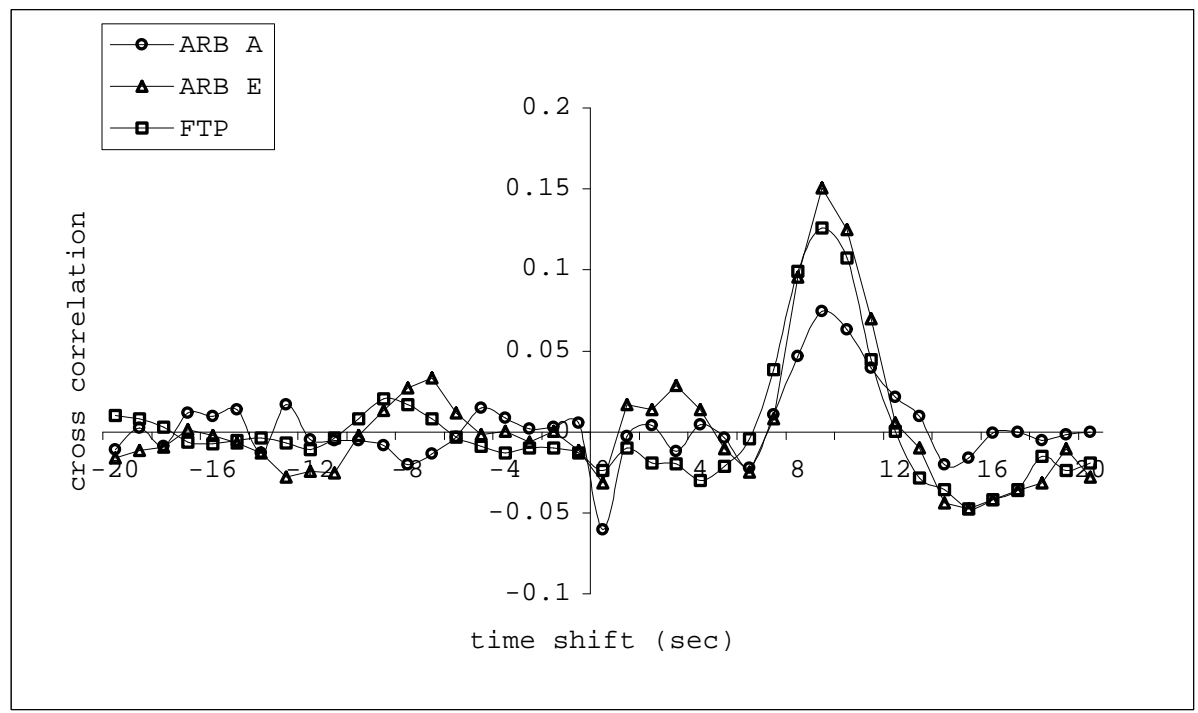

Figure 2.8b FUEL-PM first derivative cross correlation for transient tests 


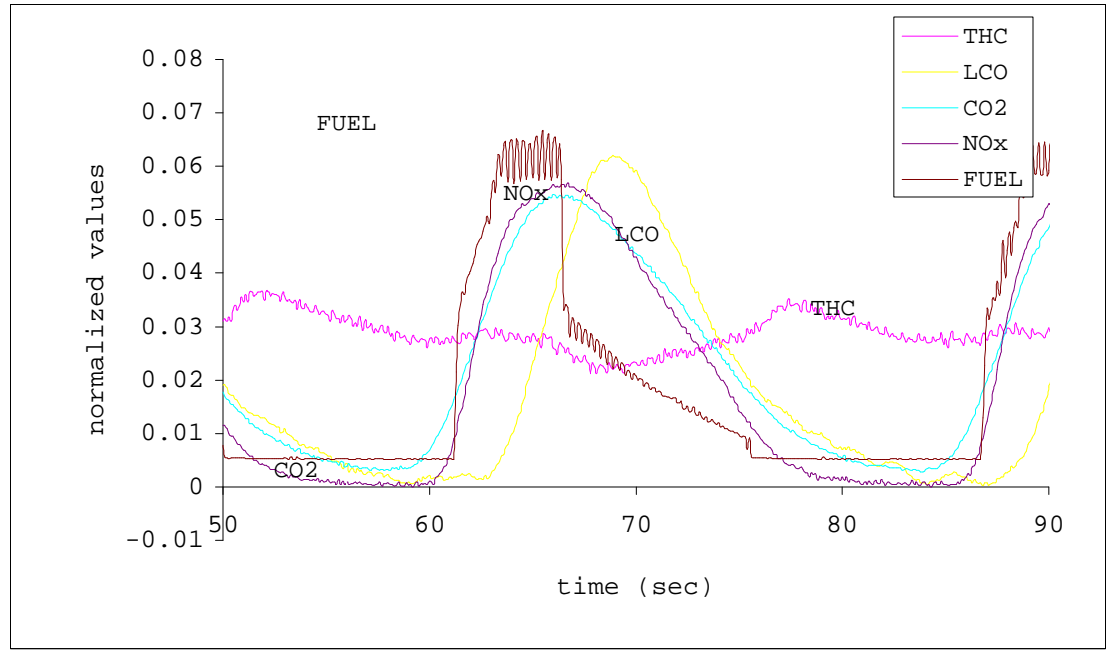

Figure 2.9a Response signals for pulsed throttle test at an engine speed of $800 \mathrm{rpm}$

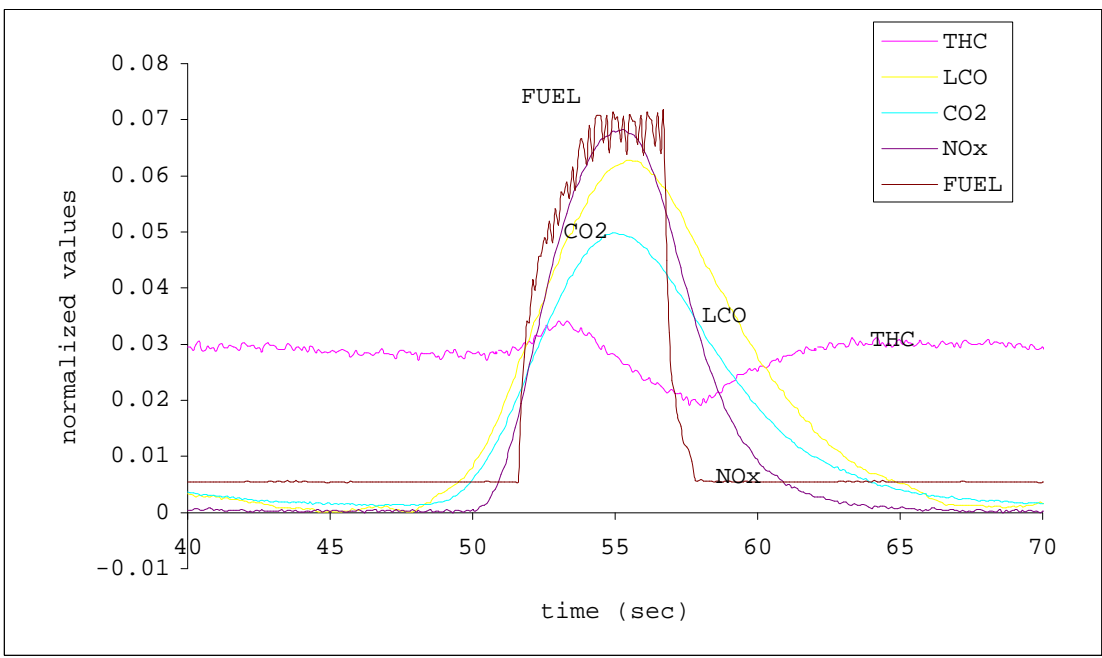

Figure 2.9b Response signals for pulsed throttle test at an engine speed of $1400 \mathrm{rpm}$

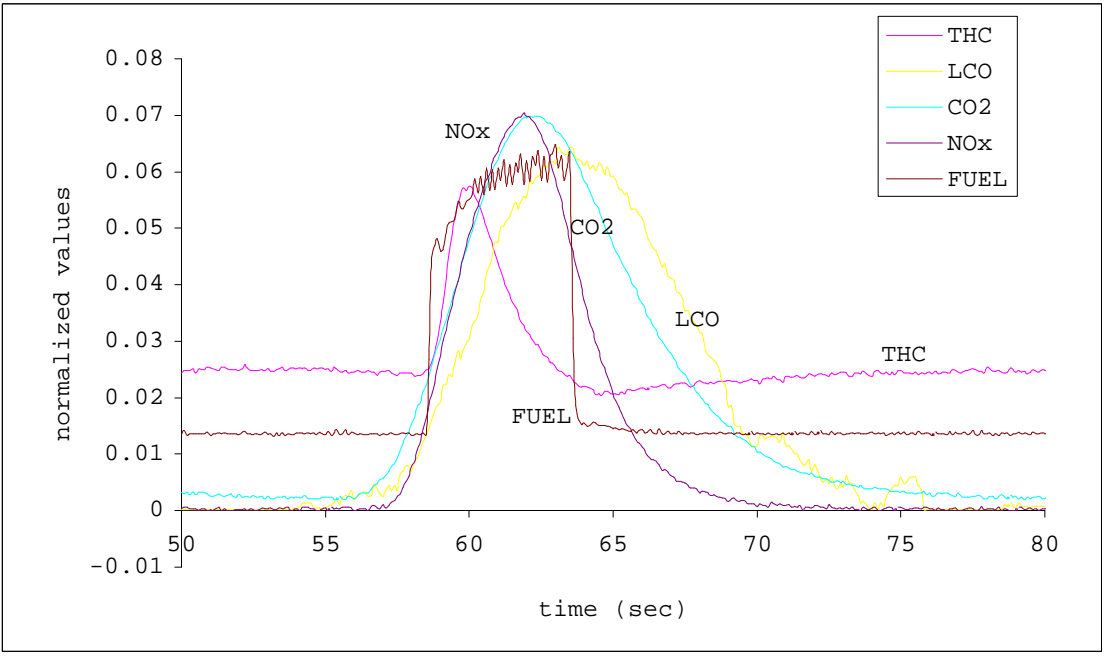

Figure 2.9c Response signals for pulsed throttle test at an engine speed of $2200 \mathrm{rpm}$ 


\section{CHAPTER 3}

\section{Neural Network-based Mean Value Engine Modeling}

\subsection{Overview}

The objective of this chapter is to develop and validate several neural networks (NN), which model various engine subsystems. This includes modeling the forward dynamics of the engine to predict its state from current and previous values of the state, $\mathrm{X}$, and control, $\mathrm{U}$, vectors. Also, several NNs are developed for the purpose of predicting engine-out exhaust emissions, which include carbon dioxide, oxides of nitrogen, carbon monoxide, total hydrocarbons, and particulate matter.

The presentation proceeds as follows: (1) conservation equations are formulated for each of the subsystems that make up the mean value model, (2) the neural network approach is described, (3) NN-based models are developed and validated, (4) the developed subsystem models are integrated and their open-loop simulation is validated.

\subsection{Mathematical Formulation}

\subsubsection{Forward Dynamics}

The main components of a turbocharged inter-cooled diesel engine are shown in Figure 3.1. A three state variable mean-value engine model can be formulated based on the following differential equations:

\section{Intake Manifold Mass Balance:}

Crankshaft Energy Balance:

$$
\frac{d}{d t}\left(\frac{P_{i m}}{T_{i m}}\right)=\left(\frac{R}{V_{i m}}\right) \dot{m}_{c}-\left(\frac{1}{120} \frac{V_{d}}{V_{i m}}\right)\left(\eta_{v} N_{e}\right)\left(\frac{P_{i m}}{T_{i m}}\right)
$$

$$
\frac{\mathrm{d}}{\mathrm{dt}}\left(\mathrm{N}_{\mathrm{e}}\right)=\left(\frac{60}{2 \pi} \frac{1}{\mathrm{I}_{\mathrm{e}}}\right)\left(\Gamma_{\mathrm{e}}-\Gamma_{\mathrm{d}}\right)
$$


Turbocharger Rotor Energy Balance:

$$
\frac{\mathrm{d}}{\mathrm{dt}}\left(\mathrm{N}_{\mathrm{tc}}\right)=\left(\frac{60}{2 \pi} \frac{1}{I_{\mathrm{tc}}}\right)\left(\Gamma_{\mathrm{t}}-\Gamma_{\mathrm{c}}\right)
$$

The various functional dependencies, $\mathrm{F}()$, of each of the static variables involved are explained below.

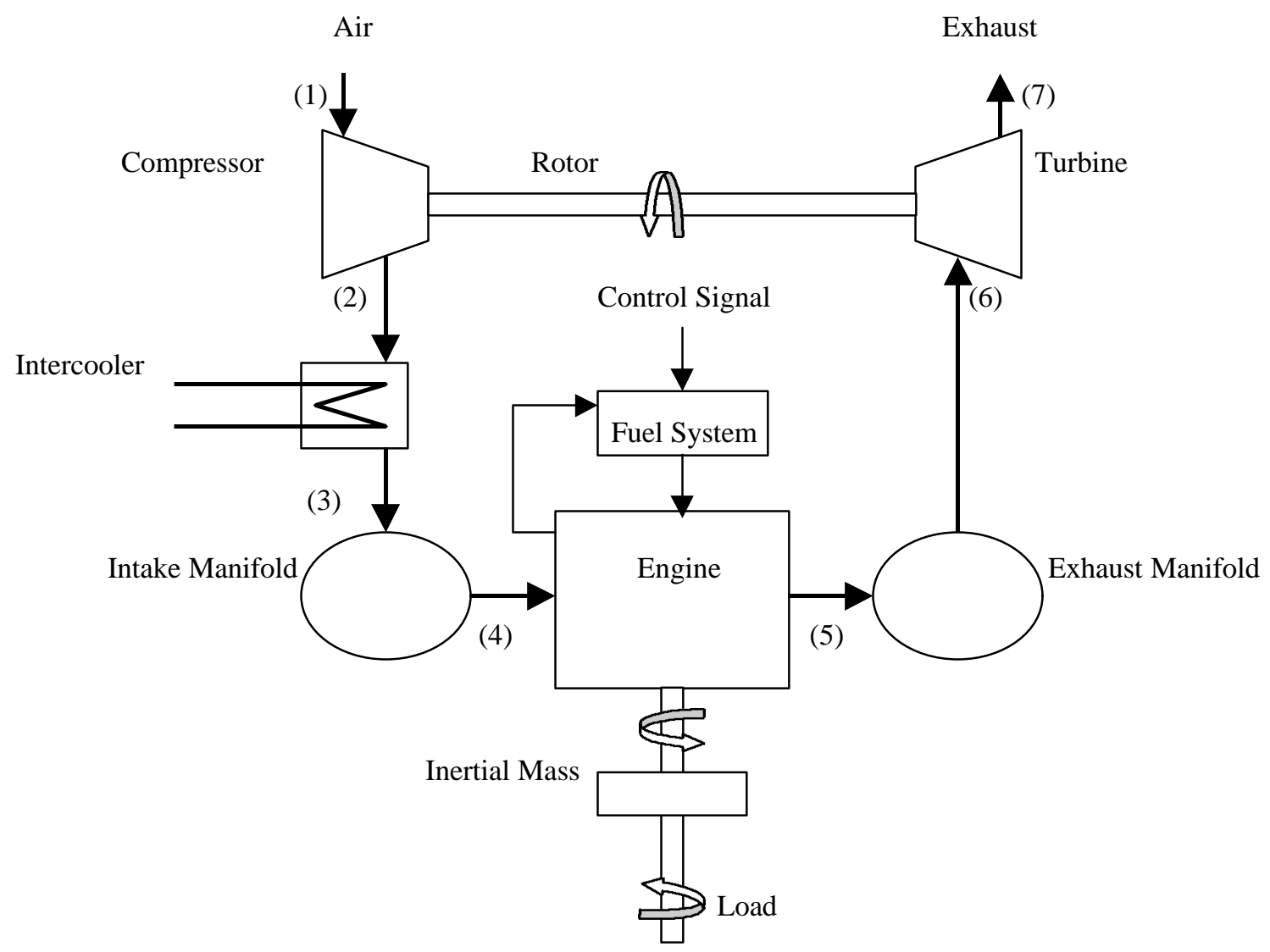

Figure 3.1 Schematic of a turbocharged inter-cooled engine

Turbocharger Compressor Model: The steady state characteristics can be expanded in terms of its mass flow rate, $m_{c}$, and its efficiency, $\eta_{c}$, as follows:

$$
\dot{\mathrm{m}}_{\mathrm{c}}=\mathrm{F}_{1}\left(\mathrm{~N}_{\mathrm{tc}}, \Pi_{\mathrm{c}}\right) \quad \eta_{\mathrm{c}}=\mathrm{F}_{2}\left(\mathrm{~N}_{\mathrm{tc}}, \Pi_{\mathrm{c}}\right)
$$


The temperature at the outlet of the compressor, $\mathrm{T}_{2}$, and the torque required to drive the compressor, $\Gamma_{\mathrm{c}}$, are given by:

$$
\begin{aligned}
& \frac{\mathrm{T}_{2}}{\mathrm{~T}_{1}}=1+\frac{1}{\eta_{\mathrm{c}}}\left(\Pi_{\mathrm{c}}^{\frac{\gamma-1}{\gamma}}-1\right)=\mathrm{F}_{3}\left(\mathrm{~N}_{\mathrm{tc}}, \Pi_{\mathrm{c}}\right) \\
& \frac{\Gamma_{\mathrm{c}}}{\mathrm{C}_{\mathrm{P}_{\mathrm{a}}} \mathrm{T}_{1}}=\frac{60}{2 \pi} \frac{\dot{\mathrm{m}}_{\mathrm{c}}}{\eta_{\mathrm{c}} \mathrm{N}_{\mathrm{tc}}}\left(\Pi_{\mathrm{c}}^{\frac{\gamma-1}{\gamma}}-1\right)=\mathrm{F}_{4}\left(\mathrm{~N}_{\mathrm{tc}}, \Pi_{\mathrm{c}}\right)
\end{aligned}
$$

where the gas state at (1), the ambient condition, is assumed constant.

Intercooler Model: Neglecting any pressure drop across the intercooler, $\left(\mathrm{P}_{\mathrm{im}}=\mathrm{P}_{3}=\mathrm{P}_{2}\right)$, the heat transfer effectiveness of the intercooler, $\varepsilon$, and its outlet temperature, $\mathrm{T}_{3}$, are given by:

$$
\varepsilon=\mathrm{F}_{5}\left(\dot{\mathrm{m}}_{\mathrm{c}}\right) \quad \frac{\mathrm{T}_{3}}{\mathrm{~T}_{1}}=(1-\varepsilon) \frac{\mathrm{T}_{2}}{\mathrm{~T}_{1}}+\varepsilon \frac{\mathrm{T}_{\mathrm{w}}}{\mathrm{T}_{1}}=\mathrm{F}_{6}\left(\mathrm{~N}_{\mathrm{tc}}, \Pi_{\mathrm{c}}\right)
$$

Integrating the compressor and intercooler equations, an expression of the compressor pressure ratio can be given by:

$$
\Pi_{c}=\frac{P_{i m}}{P_{1}}=\frac{T_{1}}{P_{1}} \frac{P_{i m}}{T_{i m}} \frac{T_{i m}}{T_{1}}=\frac{T_{1}}{P_{1}} \frac{P_{i m}}{T_{i m}} F_{6}\left(N_{t c}, \Pi_{c}\right)
$$

Since the gas thermodynamic state at (1) is constant, $\Pi_{c}$ can be given by:

$$
\Pi_{c}=F_{7}\left(N_{t c}, \frac{P_{i m}}{T_{i m}}\right)
$$


Speed-Density Model: The volumetric efficiency, $\eta_{\mathrm{v}}$, can be expressed as:

$$
\eta_{\mathrm{v}}=\mathrm{F}_{8}\left(\mathrm{~N}_{\mathrm{e}}, \frac{\mathrm{P}_{\mathrm{im}}}{\mathrm{T}_{\mathrm{im}}}\right)
$$

Turbocharger Turbine Model: The steady state characteristics can be expanded in terms of its mass flow rate, $m_{t}$, and its efficiency, $\eta_{t}$, as follows:

$$
\dot{\mathrm{m}}_{\mathrm{t}}=\mathrm{F}_{9}\left(\mathrm{~N}_{\mathrm{tc}}, \Pi_{\mathrm{t}}\right) \quad \eta_{\mathrm{t}}=\mathrm{F}_{10}\left(\mathrm{~N}_{\mathrm{tc}}, \Pi_{\mathrm{t}}\right)
$$

The torque produced by the turbine, $\Gamma_{\mathrm{t}}$ is given by:

$$
\frac{\Gamma_{\mathrm{t}}}{\mathrm{C}_{\mathrm{p}_{\mathrm{e}}} \mathrm{T}_{1}}=\frac{60}{2 \pi} \frac{\dot{\mathrm{m}}_{\mathrm{t}} \eta_{\mathrm{t}}}{\mathrm{N}_{\mathrm{tc}}} \frac{\mathrm{T}_{6}}{\mathrm{~T}_{1}}\left(1-\Pi_{\mathrm{t}}^{\frac{1-\gamma_{\mathrm{e}}}{\gamma_{\mathrm{e}}}}\right)=\mathrm{F}_{11}\left(\mathrm{~N}_{\mathrm{tc}}, \Pi_{\mathrm{t}}, \frac{\mathrm{T}_{6}}{\mathrm{~T}_{1}}\right)
$$

Substituting the expressions for $\Gamma_{\mathrm{c}}$ and $\Gamma_{\mathrm{t}}$ into the turbocharger energy balance, the following equation results:

$$
\frac{\mathrm{d}}{\mathrm{dt}}\left(\mathrm{N}_{\mathrm{tc}}\right)=\left(\frac{60}{2 \pi} \frac{\mathrm{T}_{1}}{\mathrm{I}_{\mathrm{tc}}}\right)\left(\mathrm{C}_{\mathrm{P}_{\mathrm{e}}} \mathrm{F}_{11}\left(\mathrm{~N}_{\mathrm{tc}}, \Pi_{\mathrm{t}}, \frac{\mathrm{T}_{\mathrm{f}}}{\mathrm{T}_{1}}\right)-\mathrm{C}_{\mathrm{P}_{\mathrm{a}}} \mathrm{F}_{4}\left(\mathbb{N}_{\mathrm{tc}} \mathrm{F}_{7}\left(\mathrm{~N}_{\mathrm{tc}} \frac{\mathrm{P}_{\mathrm{im}}}{\mathrm{T}_{\mathrm{im}}}\right)\right)\right)=\mathrm{F}_{12}\left(\mathrm{~N}_{\mathrm{tc}}, \frac{\mathrm{P}_{\mathrm{im}}}{\mathrm{T}_{\mathrm{im}}}, \frac{\mathrm{P}_{\mathrm{ex}}}{\mathrm{P}_{\mathrm{bk}}}, \frac{\mathrm{T}_{\mathrm{ex}}}{\mathrm{T}_{\mathrm{am}}}\right)
$$

Substituting the expressions for $m_{c}$ and $\eta_{v}$ into the manifold mass balance equation, gives:

$$
\frac{\mathrm{d}}{\mathrm{dt}}\left(\frac{\mathrm{P}_{\mathrm{im}}}{\mathrm{T}_{\mathrm{im}}}\right)=\left(\frac{\mathrm{R}}{\mathrm{V}_{\mathrm{im}}}\right) \mathrm{F}_{1}\left(\mathrm{~N}_{\mathrm{tc}}, \mathrm{F}_{7}\left(\mathrm{~N}_{\mathrm{tc}}, \frac{\mathrm{P}_{\mathrm{im}}}{\mathrm{T}_{\mathrm{im}}}\right)\right)-\left(\frac{1}{120} \frac{\mathrm{V}_{\mathrm{d}}}{\mathrm{V}_{\mathrm{im}}}\right)\left(\mathrm{F}_{8}\left(\mathrm{~N}_{\mathrm{e}}, \frac{\mathrm{P}_{\mathrm{im}}}{\mathrm{T}_{\mathrm{im}}}\right) \mathrm{N}_{\mathrm{e}}\right)\left(\frac{\mathrm{P}_{\mathrm{im}}}{\mathrm{T}_{\mathrm{im}}}\right)=\mathrm{F}_{13}\left(\mathrm{~N}_{\mathrm{e}}, \mathrm{N}_{\mathrm{tc}}, \frac{\mathrm{P}_{\mathrm{im}}}{\mathrm{T}_{\mathrm{im}}}\right)
$$

Or 


$$
\mathrm{N}_{\mathrm{tc}}=\mathrm{F}_{14}\left(\mathrm{~N}_{\mathrm{e}}, \frac{\mathrm{P}_{\mathrm{im}}}{\mathrm{T}_{\mathrm{im}}}, \frac{\mathrm{d}}{\mathrm{dt}}\left(\frac{\mathrm{P}_{\mathrm{im}}}{\mathrm{T}_{\mathrm{im}}}\right)\right.
$$

Differentiating the above equation with respect to time,

$$
\frac{d}{d t}\left(N_{t c}\right)=F_{15}\left(N_{e}, \frac{d}{d t}\left(N_{e}\right), \frac{P_{i m}}{T_{i m}}, \frac{d}{d t}\left(\frac{P_{i m}}{T_{i m}}\right), \frac{d^{2}}{d t^{2}}\left(\frac{P_{i m}}{T_{i m}}\right)\right.
$$

Equating $\mathrm{F}_{15}()$ with $\mathrm{F}_{12}()$ and substituting for $\mathrm{N}_{\mathrm{tc}}$ using $\mathrm{F}_{14}()$, the manifold dynamics dependencies are given as follows:

$$
\frac{d^{2}}{d t^{2}}\left(\frac{P_{i m}}{T_{i m}}\right)=F_{16}\left(N_{e}, \frac{d}{d t}\left(N_{e}\right), \frac{P_{i m}}{T_{i m}}, \frac{d}{d t}\left(\frac{P_{i m}}{T_{i m}}\right) \frac{P_{e x}}{P_{b k}}, \frac{T_{e x}}{T_{a m}}\right)
$$

Furthermore, it is possible to express the exhaust manifold state in terms of the engine state vector, $\mathrm{X}$, and the control vector, $\mathrm{U}$, as follows:

$$
\frac{\mathrm{T}_{\mathrm{ex}}}{\mathrm{T}_{\mathrm{am}}}=\mathrm{F}_{17}(\mathrm{X}, \mathrm{U}) \quad \frac{\mathrm{P}_{\mathrm{ex}}}{\mathrm{P}_{\mathrm{bk}}}=\mathrm{F}_{18}(\mathrm{X}, \mathrm{U}) \quad \mathrm{X}^{\mathrm{T}}=\left[\mathrm{N}_{\mathrm{e}}, \frac{\mathrm{P}_{\mathrm{im}}}{\mathrm{T}_{\mathrm{im}}}\right]
$$

Finally, the manifold dynamics can be expressed as:

$$
\frac{d^{2}}{d t^{2}}\left(\frac{P_{i m}}{T_{i m}}\right)=F_{19}\left(N_{e}, \frac{d}{d t}\left(N_{e}\right), \frac{P_{i m}}{T_{i m}}, \frac{d}{d t}\left(\frac{P_{i m}}{T_{i m}}\right), U\right)=F_{19}\left(X, \frac{d X}{d t}, U\right)
$$


The engine brake torque, $\Gamma_{\mathrm{e}}$, can be expressed as follows:

$$
\Gamma_{\mathrm{e}}=\mathrm{F}_{20}\left(\mathrm{~N}_{\mathrm{e}}, \frac{\mathrm{P}_{\mathrm{im}}}{\mathrm{T}_{\mathrm{im}}}, \mathrm{U}\right)=\mathrm{F}_{20}(\mathrm{X}, \mathrm{U})
$$

Substituting in the engine crankshaft energy balance, the engine speed dynamics could be expressed as:

$$
\frac{\mathrm{d}}{\mathrm{dt}}\left(\mathrm{N}_{\mathrm{e}}\right)=\left(\frac{60}{2 \pi} \frac{1}{\mathrm{I}_{\mathrm{e}}}\right)\left(\mathrm{F}_{20}(\mathrm{X}, \mathrm{U})-\Gamma_{\mathrm{d}}\right)
$$

\subsubsection{Exhaust Emissions}

The mechanisms of the engine exhaust emissions formation are highly complex in a heterogeneous combustion environment such as that found in the diesel engine. The is due to the fact that it occurs in an open system with movable boundaries and complex geometry, operating at high temperatures and pressures, and it retains a memory of the preceding cycle with random cycle-by-cycle variations. The cycle time constant varies with engine speed and the combustion mechanisms involved are very complex. Some of the chemical reaction time scales are comparable to the cycle repetition rate.

In order to derive the functional dependencies of emissions on engine state and control variables, one needs to consider the governing partial-differential conservation equations of mass, momentum, energy, and individual chemical species. Furthermore, in-cylinder turbulence needs to be considered as it has a deterministic effect on combustion, especially in the case of internal combustion engines where flow reversals and swirl occur. These complex interactions prohibit any attempt at identifying appropriate functional dependencies for emissions formation 
and would simply result in inclusion of all the measured variables. Therefore a heuristic approach has been used to model emissions formation and a general functional dependency of the $\mathrm{i}^{\text {th }}$ pollutant, $\mathrm{E}_{\mathrm{i}}$, is given by:

$$
E_{i}=F_{i}\left(N_{e}, \frac{P_{i m}}{T_{i m}}, U\right)=F_{i}(X, U)
$$

On the time scale considered by the mean value approach, engine-out emissions can be safely classified as instant 'static' variables since the time scale involved in their formation is much less than a few engine cycles. During transient operation, measurement of engine-out emissions on these time scales is difficult since it requires fast response analyzers capable of resolving cycle by cycle variations. Though such analyzers exist for emissions of hydrocarbons and recently for nitric oxide, no equivalent fast response analyzers currently exist for carbon monoxide or particulate matter.

For the current investigation, the emissions measurement system features constant volume sampling using a full-scale dilution tunnel where exhaust gas and dilution air are pulled by a blower through a metering venturi operating at a choked condition. The distortion of each measured emissions signal by the sampling system due to axial dispersion was clearly demonstrated in the previous chapter. Since no attempt was made to de-convolute the emissions signals, one needs to consider this effect in the emissions mean value model. This effect can be accounted for by including a sliding time window of the engine state and control parameters as input to the model. Thus, in effect the engine-tunnel-analyzer system is modeled as single, consolidated unit. This facilitates greatly the model validation procedure at the expense of some degradation in the transient emissions prediction, which is acceptable if integral results (emissions integrated across a whole transient cycle) are of primary interest. An alternative 
approach is to resort to steady state testing, thus in effect eliminating the tunnel-analyzer effect. The main drawbacks of this approach are prolonged testing procedure and difficulty in validating the model predictions under transient operation. System identification can be used to derive digital filters capable of reconstructing the original signal from the measured (distorted) one, but this approach was not employed here. 


\subsection{Neural Network Approach}

An overview of the basic architecture used is given followed by the mathematical formulation of the parameter adjustment algorithms used.

\subsubsection{Overview}

The basic neural network (NN) architecture used in the current investigation is shown in Figure 3.2. The notation introduced by Hagan et al. (1996) has been used. It features a feedforward fully connected two-layer NN with a non-linear activation hidden layer.

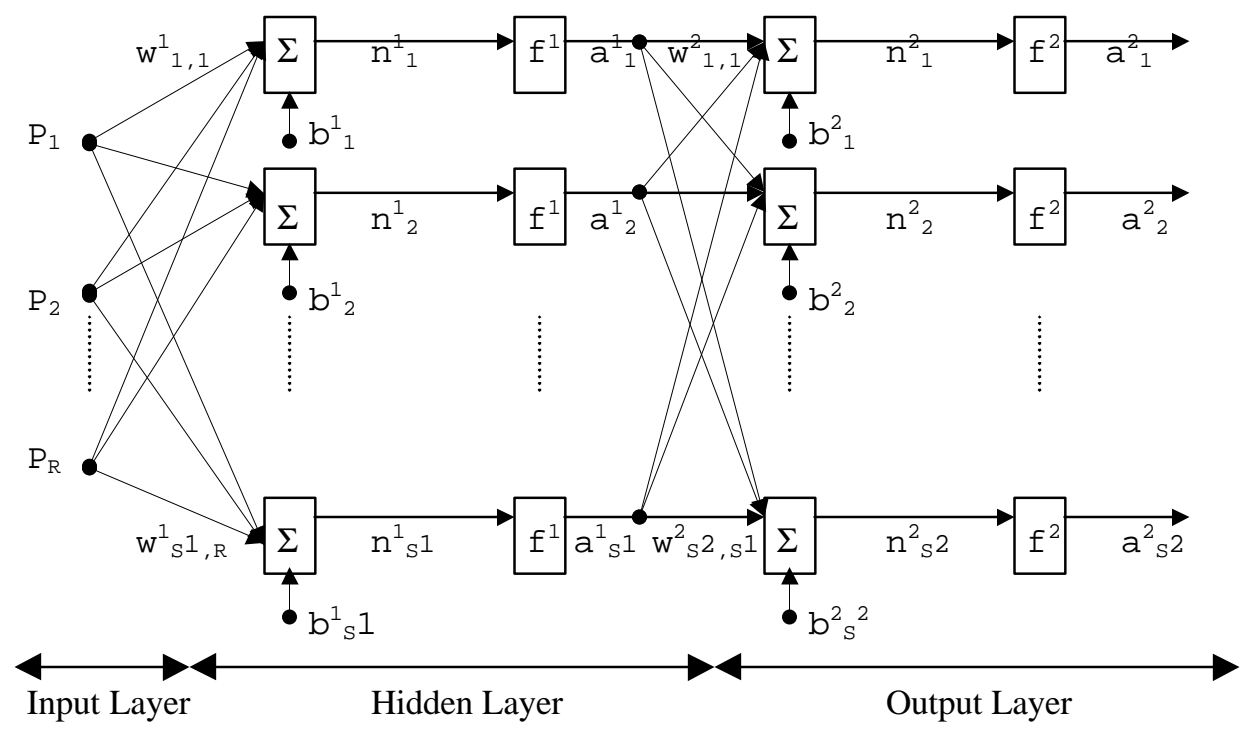

Figure 3.2 Two-layer fully-connected feedforward neural network

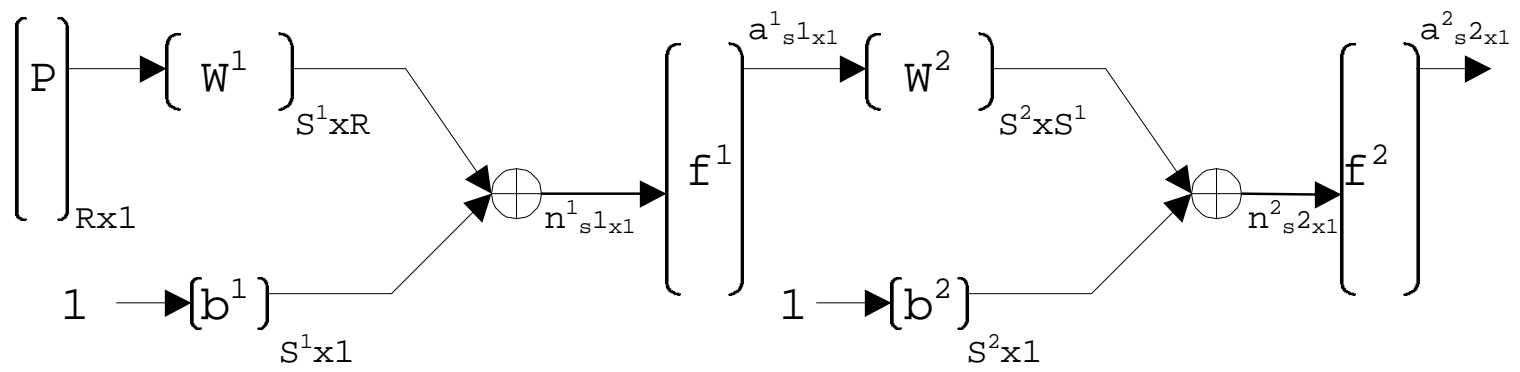

Figure 3.3 Matrix representation of a two-layer neural network 
where $[\mathrm{P}]$ is the input vector. For the $\mathrm{m}^{\text {th }}$ layer, $\left[\mathrm{W}^{\mathrm{m}}\right]$ is the matrix of weights, $\left[\mathrm{b}^{\mathrm{m}}\right]$ is the bias vector, and $\mathrm{s}$ is the number of neurons.

The $\mathrm{m}^{\text {th }}$ layer output for an M layer NN is calculated by:

$$
a^{m+1}=f^{m+1}\left(W^{m+1} \cdot a^{m}+b^{m+1}\right) \text { for } m=0,1, \ldots ., M-1 \text { and } a^{\circ}=P
$$

The output layer can employ either linear or non-linear activation. The non-linear activation function used here is exclusively the hyperbolic tangent 'tanh', which is defined as follows:

$$
\tanh (\mathrm{x})=\frac{2}{1+\mathrm{e}^{-2 \mathrm{x}}}-1
$$

The $\tanh ()$ function has the effect of confining its input argument to output values between -1 and +1 for all values of the input, as shown in Figure 3.4.

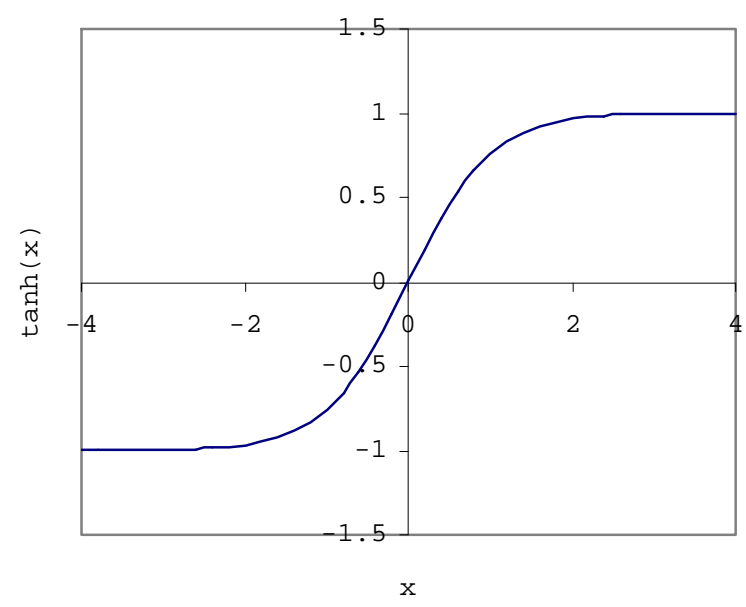

Figure 3.4 Non-linear activation hyperbolic tangent function

The motivation for using a single hidden layer architecture is based on its proven ability to approximate virtually any continuous function, provided sufficiently many hidden neurons are used (Hornik et al., 1989). Linear activation is used in the output layer in order to allow the NN 
to generalize or to extrapolate to output values outside their pre-set minimum and maximum values, otherwise non-linear activation is used in the output layer.

The NN architecture shown in Figure 3.2 can be used to approximate non-linear mapping which is either static (non-temporal) or dynamic (temporal) between the input and the output vectors. Dynamic representation is induced by feeding the NN time delayed values of the current time step input and/or output vectors. This time-delayed NN architecture is commonly referred to as partially recurrent since only the output layer values are fed back through a tapped delay line into the NN input vector. In general, the output of the $\mathrm{NN}$ at time $(\mathrm{k}+1)$ depends both on the past $\mathrm{n}$ values of the output vector as well as the past $\mathrm{m}$ values of the input vector, as shown in Figure 3.5, (Narendra and Parthasarathy, 1990).

System identification can proceed in two modes, either parallel or parallel-series modes. In the parallel identification model, the $\mathrm{NN}$ estimated output vector is fed back through the input vector. On the other hand, in the series-parallel mode the actual system output (rather than the NN output) is fed back into the identification NN, as shown in Figure 3.5. The series-parallel mode is preferred over the parallel mode, when possible, since it enjoys the advantage that all signals used in the identification procedure are bounded for the case of a stable actual system (Narendra and Parthasarathy, 1990). On the other hand, for the case of off-line simulation, one has to revert to parallel mode since actual system outputs are not available.

During the system identification phase, whether a static or a dynamic system, the used NN parameters (weights and biases) need to be adjusted in order to minimize certain performance criteria, for example the sum of the square of the differences between the actual measured plant (engine) and network output values. 


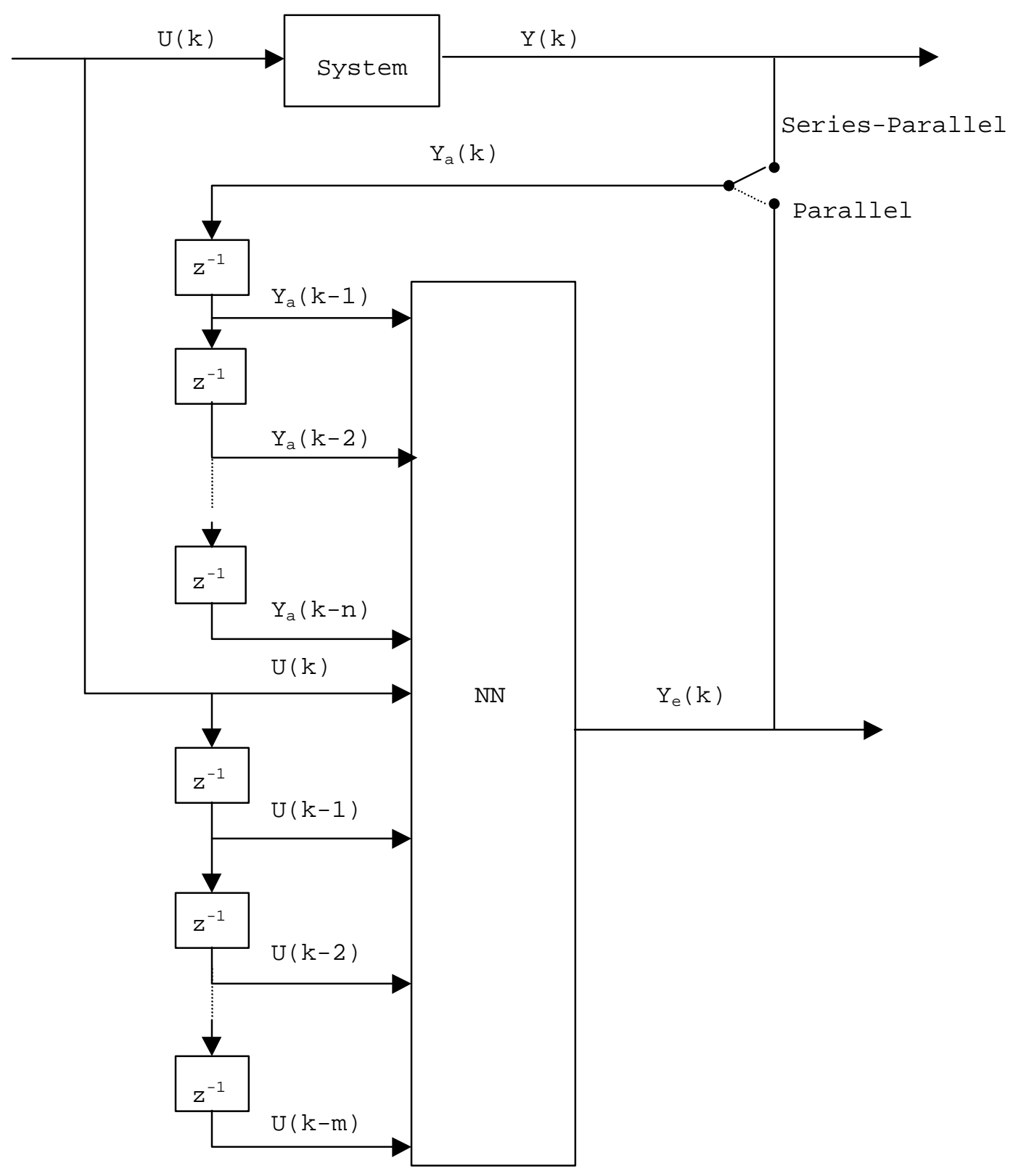

Figure 3.5 Dynamic identification with a partially recurrent neural network

The NN parameter adjustment can proceed either off-line in a batch mode where its parameters are only updated after all of the input-output pairs have been presented, or on-line where incremental training is used to adapt the $\mathrm{NN}$ parameters such as for the purpose of on-line control. For both cases, gradient descent algorithms are employed where back-propagation can be used for on-line adaptation and standard numerical optimization techniques can be used for 
off-line training. At the start of training, the NN parameters are initialized using small (non-zero) random values because the origin of the parameters' space tends to be a saddle point for the performance surface, which has very flat regions for large parameter values (Hagan et al., 1996). In the following two sections, a mathematical formulation for the parameter adjustment algorithms used is provided.

\subsubsection{Back-Propagation Algorithm}

Back-propagation employs a gradient descent algorithm used to adjust the network parameters (weights and biases) in order to minimize the performance index given by:

$$
F(x)=e^{T}(x) \cdot e(x)
$$

where $\mathrm{x}$ is the vector of the network weights and biases and e is the error vector, which is defined as the desired output, $t$, minus the NN output, a, for a training data set of input-output pairs [p,t].

For an $\mathrm{NN}$ with $\mathrm{M}$ layers and $\mathrm{s}^{\mathrm{m}}$ neurons in the $\mathrm{m}^{\text {th }}$ layer, the back-propagation algorithm employs the following steps recursively (Hagan et al., 1996):

Forward Step: evaluate the network $\mathrm{m}^{\text {th }}$ layer output vector, $\mathrm{a}^{\mathrm{m}}$, and the Jacobian matrix, $\mathrm{dF}^{\mathrm{m}}$, which is calculated using the chain rule of calculus as follows:

$$
\begin{aligned}
& \mathrm{a}^{\circ}=\mathrm{P} \\
& \text { for } m=0,1, . . ., M-1 \\
& \mathrm{n}^{\mathrm{m}+1}=\mathrm{W}^{\mathrm{m}+1} \cdot \mathrm{a}^{\mathrm{m}}+\mathrm{b}^{\mathrm{m}+1} \\
& a^{m+1}=f^{m+1}\left(n^{m+1}\right) \\
& d F^{m}=\text { diagonal }\left(\mathrm{df}^{\mathrm{m}}\left(\mathrm{n}^{\mathrm{m}}{ }_{i}\right) \quad i=1,2, \ldots, \mathrm{s}^{\mathrm{m}}\right) \\
& \text { end }
\end{aligned}
$$

where $\mathrm{df}$ is the derivative of the activation function with respect to its argument and the approximation error vector, e, is given by:

$$
e=\left(t-a^{\mathrm{M}}\right)
$$

Backward Step: evaluate the $\mathrm{m}^{\text {th }}$ layer parameter sensitivity, $\mathrm{S}^{\mathrm{m}}$, to the approximation error, e, as follows:

$$
\begin{aligned}
& \mathrm{S}^{\mathrm{M}}=-2\left[\mathrm{dF} \mathrm{F}^{\mathrm{M}}\right][\mathrm{e}] \\
& \text { for } \mathrm{m}=\mathrm{M}-1, \mathrm{M}-2, . . ., 1
\end{aligned}
$$




$$
\text { end } S^{m}=\left[d F^{m}\right]\left[W^{m+1}\right]^{T}\left[S^{m+1}\right]
$$

Update Step: increment the $\mathrm{m}^{\text {th }}$ layer weights, $\mathrm{W}^{\mathrm{m}}$, and biases, $\mathrm{b}^{\mathrm{m}}$, using the following updates:

$$
\begin{aligned}
& \text { For } \mathrm{m}=1,2, \cdot \cdot \cdot, \mathrm{M} \\
& \Delta \mathrm{W}^{\mathrm{m}}=-\lambda\left[\mathrm{S}^{\mathrm{m}}\right]\left[\mathrm{a}^{\mathrm{m}-1}\right]^{\mathrm{T}} \\
& \Delta \mathrm{b}^{\mathrm{m}}=-\lambda\left[\mathrm{S}^{\mathrm{m}}\right] \\
& \text { end }
\end{aligned}
$$

where the adjustable learning rate parameter, $\lambda$, value is between 0 and 1 .

For the case of a single layer $\mathrm{NN}$, the performance surface is quadratic with a single stationary point and the function contours are elliptic because the Hessian matrix is constant. Thus, backpropagation reduces to a least squares algorithm and convergence to the single minimum point is guaranteed. However, the performance surface for a multi-layered network may have many local minimum points, and the curvature can vary widely in different regions of the parameter space. This results in the infamous local minimum problem when adapting a multi-layer NN using back-propagation, where the algorithm may get trapped in one of these local minimum points. It also results in variable convergence rate trajectories with long periods of little progress followed by short periods of rapid advance, typical of the back-propagation algorithm. Improvement to back-propagation is possible through heuristic approaches (e.g. adding a momentum term or using variable learning rate) or methods based on standard numerical optimization techniques (e.g. conjugate gradient or Newton's method). The main disadvantage of the heuristic techniques is that they require several parameters to be set and convergence is not guaranteed. In the next section the Levenberg-Marquard algorithm is briefly formulated. 


\subsubsection{Levenberg-Marquardt Algorithm}

The Levenberg-Marquardt algorithm is a variation of Newton's method, which makes use of the second derivatives of the performance index (Hessian matrix) to achieve second-order convergence criteria. For the case of performance index in the form of a sum of squares, the Hessian matrix, $\mathrm{d}^{2} \mathrm{~F}$, can be approximated in terms of the Jacobian matrix, $\mathrm{dF}$, as follows (Hagan et al., 1996):

$$
d^{2} F=2[d F]^{T}[d F]
$$

where $\mathrm{dF}$ is the derivatives of the errors vector, instead of the derivatives of the squared errors for the case of back-propagation.

The increment updates for the network weights and biases vector, $\mathrm{x}$, are given by the LevenbergMarquardt algorithm as follows:

$$
\Delta x=-\left(d F^{T} d F+\mu I\right)^{-1} d F^{T} e
$$

where $\mathrm{I}$ is the identity matrix and $\mu$ is a scalar. When $\mu$ is set to zero, the update formulation reduces to Netwon's method with the approximated Hessian matrix. When $\mu$ is large, this becomes the familiar back-propagation with a small learning rate $\lambda=1 / \mu$. Since the Newton method achieves fast convergence in the vicinity of a minimum, the idea is to shift as soon as possible towards Newton's method by decreasing the value of $\mu$, which is initially set to a high value to guarantee convergence.

The Jacobian matrix, $\mathrm{dF}$, is defined as the partial derivative of the error vector, e, with respect to the parameter vector, $\mathrm{x}$, where

$$
\begin{aligned}
& e^{T}=\left[\begin{array}{llll}
e^{1} & e^{2} \cdot \cdot \cdot e^{q} \cdot \cdot \cdot e^{Q}
\end{array}\right] \\
& x^{T}=\left[\begin{array}{llllll}
W^{1} & b^{1} & W^{2} & b^{2} \cdot \cdot \cdot W^{m} & b^{m} \cdot \cdot \cdot W^{M} & b^{M}
\end{array}\right]
\end{aligned}
$$


The sensitivity vector, $\mathrm{S}_{\mathrm{q}}{ }^{\mathrm{M}}$, for the $\mathrm{m}^{\text {th }}$ layer and the $\mathrm{q}^{\text {th }}$ input vector is computed as follows (Hagan and Menhaj, 1994):

$$
\begin{aligned}
& \text { for } q=1,2, \ldots, \cdot, Q \\
& \mathrm{~S}_{\mathrm{q}}^{\mathrm{M}}=-\left[\mathrm{dF} \mathrm{F}^{\mathrm{M}}\right] \\
& \text { for } \mathrm{m}=\mathrm{M}-1, \mathrm{M}-2, \text {. . . , } 1 \\
& \mathrm{~S}_{\mathrm{q}}{ }^{\mathrm{m}}=\left[\mathrm{dF}^{\mathrm{m}}\right]\left[\mathrm{W}^{\mathrm{m}+1}\right]^{\mathrm{T}}\left[\mathrm{S}_{\mathrm{q}}{ }^{\mathrm{m}+1}\right] \\
& \text { end } \\
& \text { end }
\end{aligned}
$$

For every input vector applied to the network there will be $S^{\mathrm{M}}$ errors and for each error there will be one row of the Jacobian matrix. The Jacobian matrix, $\mathrm{dF}$, is given by:

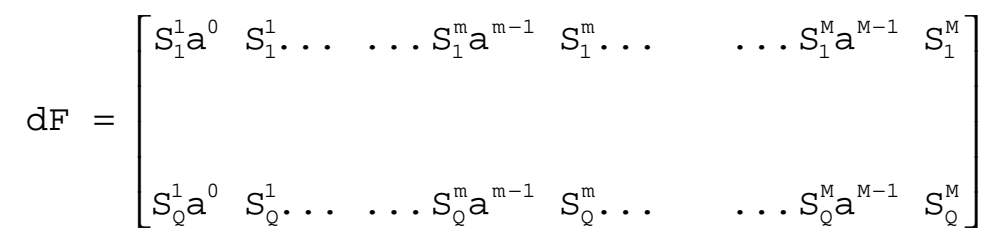

The convergence criteria are either the sum of squared errors or the norm of the gradient, $2[\mathrm{dF}]^{\mathrm{T}}[\mathrm{e}]$, being reduced to some tolerance value. 


\subsection{Engine Model Development and Validation}

Different subsystems, which constitute the mean value engine model, are shown in Figure 3.6. In view of the mathematical formulation given in Section 3.2, the elements of the engine state vector, $\mathrm{X}$, consisted of engine operating mode, engine speed in revolutions per minute, and air mass flow rate in milligrams per cycle.

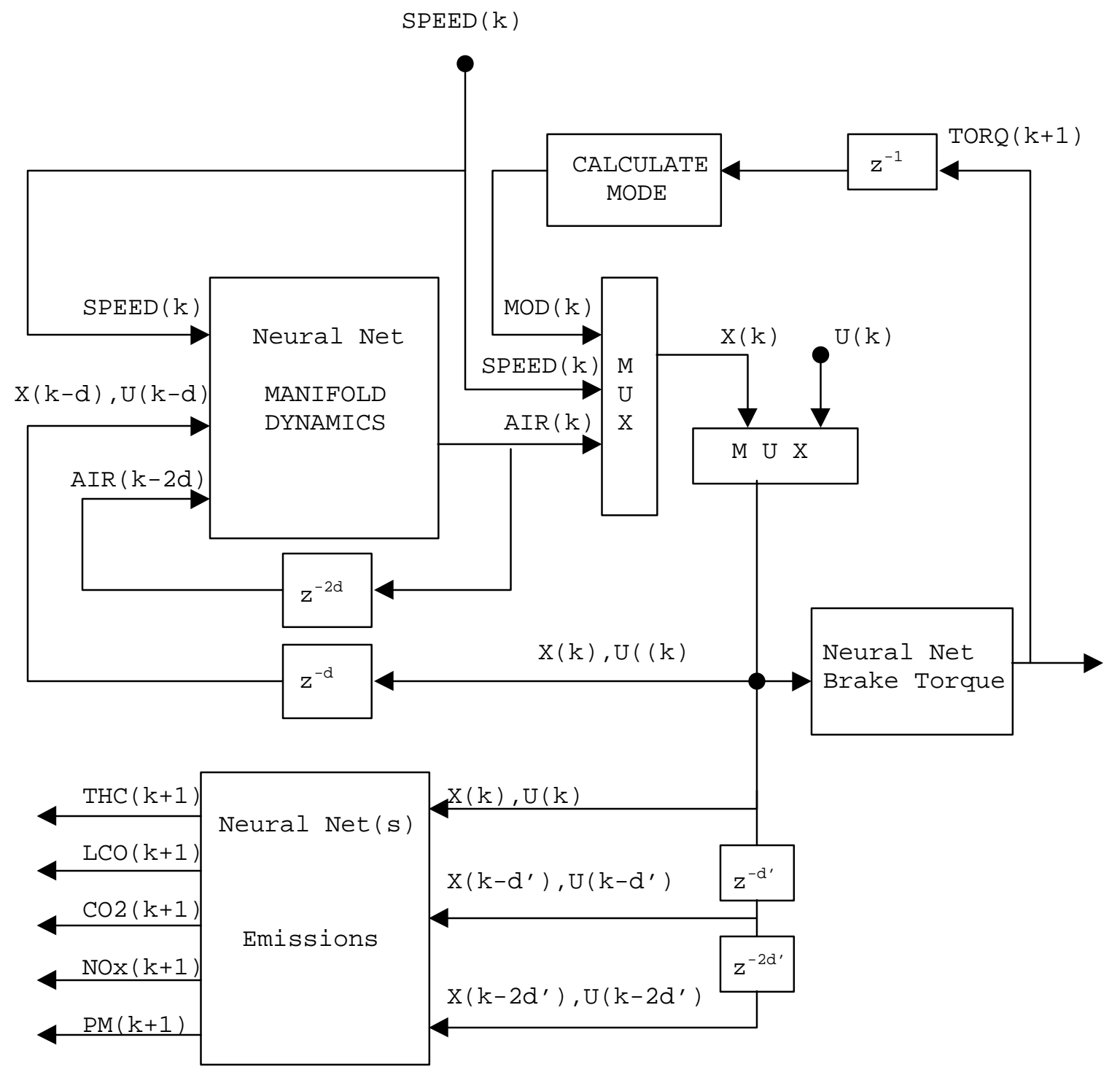

Figure 3.6 Block diagram of the open-loop mean value engine model 
The engine mode of operation acted as a three-state switch (motored, idle, or fired), and was set equal to the sign of the engine brake mean effective pressure where the idle minimum and maximum bounds were set at \pm 0.1 bar. The inclusion of engine operating mode as a state variable enhanced the prediction accuracy of torque and exhaust gas emissions under motoring and idle operation because their values were much lower than those encountered during fired operation.

The air mass flow rate was calculated from the intake manifold pressure and air temperature as follows:

$$
\dot{\mathrm{m}}_{\mathrm{a}}=\left(\frac{1}{120} \frac{\mathrm{V}_{\mathrm{d}}}{\mathrm{R}}\right)\left(\eta_{\mathrm{v}} \mathrm{N}_{\mathrm{e}}\right)\left(\frac{\mathrm{P}_{\mathrm{im}}}{\mathrm{T}_{\mathrm{im}}}\right)
$$

The volumetric efficiency was assumed to be constant and set equal to unity for all engine operation. The assumption of unity volumetric efficiency relied on the fact that the test engine was throttle-less. Also, the explicit functional dependence of the volumetric efficiency on the state of the engine was accounted for in the mathematical formulation derived in Section 3.2. Using air flow rate as a state variable, rather than the manifold air pressure, eliminated the need for a temperature correction and augmented the modeling of the naturally coupled intercooler and turbocharger subsystems. The control variable vector, U, consisted of fuel flow rate (in milligrams per cycle), injection timing (in degree before top dead center), and injection control pressure (in MPa).

Two neural networks were used to predict the time development of the engine state variable, as shown in Figure 3.6. They served the purpose of identifying the turbocharger and the combustion efficiency. The first NN was partially recurrent and was used to model the dynamics of the intake air mass flow rate as a function of previous values of the state and control vectors, current 
engine speed, and further delayed value of air mass flow rate. The second NN was static and modeled the engine brake-torque as a function of the state and control vectors. Several NNs were used to predict engine-out emissions as a function of a sliding window of state and control vectors, as shown in Figure 3.6. The exhaust emissions considered were carbon dioxide, oxides of nitrogen, carbon monoxide, total hydrocarbons, and particulate matter.

The NN parameters (weights and biases) were adjusted to minimize the sum of the squares of the differences between the actual plant and network output values. The NN was trained off-line in a batch mode where its parameters were only updated after all of the input-output pairs were presented. The arbitrary cycle data, cycles A through $\mathrm{H}$, were used in the training procedure and the FTP cycle data was used to validate the trained NN generalization capability. The Levenberg-Marquardt algorithm was employed for off-line training and the target performance goal (mean square difference between $\mathrm{NN}$ output and target output) was arbitrarily set at 0.001 . The maximum number of training epochs (presentation of the input/output pairs and the adjustment of NN parameters) was set at 100 regardless of whether the target performance goal was achieved or not. The Levenberg-Marquardt algorithm was very efficient in reaching a performance goal, defined as the root mean square error, two orders of magnitude lower than its initial value in less than 10 training epochs, as shown in Figure 3.7 for the case of predicting engine brake torque. In general, training the NN for more than 100 epochs did not result in any appreciable improvement in prediction signifying the need for more neurons in the hidden layer when the NN input vector was appropriately selected.

The basic NN architecture used was a single hidden layer with non-linear activation, as described in the previous section, and the number of neurons was set initially to four. If proven insufficient to achieve the desired prediction accuracy, the number of neurons was increased. 
Adjusting the sliding window span of the input vector proved to be very useful in enhancing the prediction accuracy in the case of emissions prediction, especially when increasing the number of neurons in the hidden layer did not result in any significant improvement. In this respect, using several NNs for emissions prediction allowed the flexibility to adjust the sliding window size and number of neurons for each individual pollutant. This is contrasted to one single massive-architecture NN where the individual pollutant prediction accuracy is limited to the least accurate prediction. The NN architectures used are shown in Table 3.1.

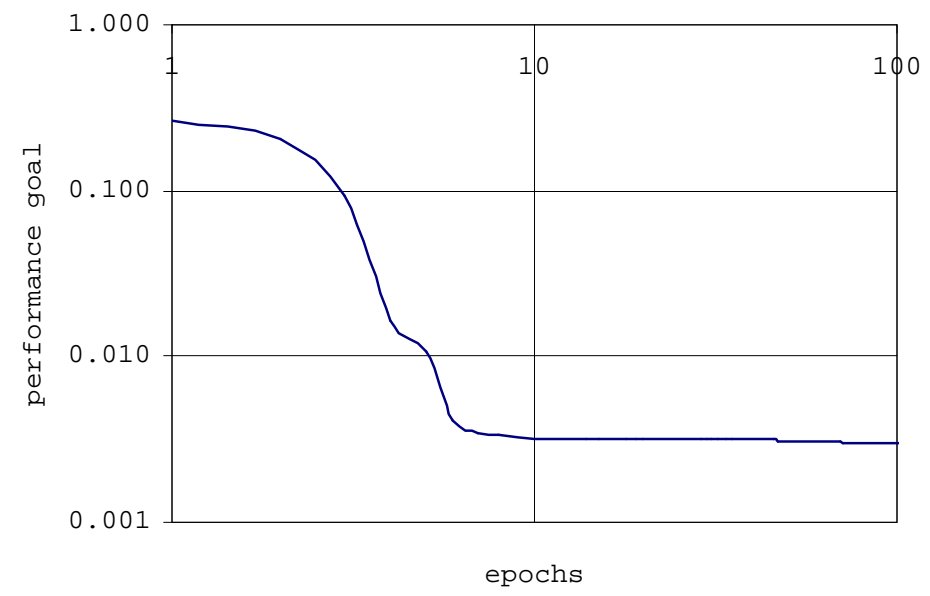

Figure 3.7 Training history for engine brake torque prediction

In order to speed up the rate of convergence and guarantee bounded output, the data was scaled between -1 and 1 using appropriate upper and lower limits, shown in Table 3.2, over the measurements conducted. To prevent over-fitting and to improve the generalization capability of the trained $\mathrm{NN}$, early termination of training was employed using a validation data set, which was not included in the training set used for computing the gradient and updating the $\mathrm{NN}$ weights and biases. The error on the validation set was monitored during training, which was terminated when the validation error exceeded a preset tolerance value. 
Table 3.1 Neural network architectures

\begin{tabular}{|c|c|c|c|c|}
\hline & \multicolumn{3}{|c|}{ Number of Nodes } & Window \\
\hline & Input & Hidden & Output & $(\mathrm{sec})$ \\
\hline Air flow rate & 8 & 4 & 1 & 0.5 \\
\hline Brake torque & 7 & 4 & 1 & 0 \\
\hline Carbon dioxide & 18 & 4 & 1 & 2 \\
\hline Oxides of nitrogen & 18 & 4 & 1 & 2 \\
\hline Particulate matter & 18 & 16 & 1 & 10 \\
\hline Carbon monoxide & 18 & 6 & 1 & 5 \\
\hline Total hydrocarbons & 18 & 6 & 1 & 5 \\
\hline
\end{tabular}

Table 3.2 Minimum and maximum bounds of model variables

\begin{tabular}{|c|c|c|c|}
\hline & Unit & Min & $\operatorname{Max}$ \\
\hline Mode of operation & $\mathrm{n} / \mathrm{a}$ & -1 & 1 \\
\hline Engine speed & $\mathrm{rpm}$ & 600 & 3000 \\
\hline Air flow rate & $\mathrm{mg} / \mathrm{cyc}$ & 7500 & 15500 \\
\hline Fuel flow rate & $\mathrm{mg} / \mathrm{cyc}$ & 10 & 550 \\
\hline Injection timing & deg btdc & 0 & 20 \\
\hline Injection pressure & $\mathrm{MPa}$ & 0 & 20 \\
\hline Brake torque & $f t-1 b f$ & -400 & 750 \\
\hline Brake power & $\mathrm{hp}$ & -150 & 350 \\
\hline Carbon dioxide & $g / h$ & 0 & 115000 \\
\hline Oxides of nitrogen & $\mathrm{g} / \mathrm{h}$ & 0 & 1000 \\
\hline Particulate matter & $g / h$ & -50 & 50 \\
\hline Carbon monoxide & $g / h$ & 0 & 2000 \\
\hline Total hydrocarbons & $\mathrm{g} / \mathrm{h}$ & 0 & 100 \\
\hline
\end{tabular}

For all raw measurements, the data-sampling rate was selected based on the time scale for 5 engine cycles $(0.2 \mathrm{sec}$ at $3000 \mathrm{rpm}$ to $1.0 \mathrm{sec}$ at $600 \mathrm{rpm})$, which characterized the engine dynamic behavior in the mean value approach. The sampling rate was set at $10 \mathrm{~Hz}$, above the Nyquist frequency bound $(\sim 9 \mathrm{~Hz})$ for this engine which was rated at $2600 \mathrm{rpm}$.

The trained NNs, which constitute the mean value model, were validated in two stages. Firstly, each sub-model NN was simulated individually in a series-parallel mode where actual 
measurements were used as inputs. In the second stage, the engine dynamics NNs were integrated and coupled with the emissions prediction NNs. Open loop simulation was conducted in a parallel mode where estimated engine state vector was recursively used as input. The inputs to the simulation were the engine speed and control vector trajectories. The MATLAB neural network toolbox was used for training as well as simulation purposes.

The results for the first validation step are shown in Figures 3.8 through 3.14 for a portion of the FTP cycle, which was not included in the training set as mentioned earlier. The airflow and torque predictions were in very good agreement with the actual values as shown in Figures 3.8 and 3.9. The emissions prediction of carbon dioxide $\left(\mathrm{CO}_{2}\right)$ and oxides of nitrogen (NOx) matched the measurement with high overall accuracy, as shown in Figures 3.10 and 3.11, where prediction errors were highest during sharp transients. The predictions for particulate matter (PM) showed the correct trend over the highly transient operation of the FTP cycle, although not as accurate as that for $\mathrm{CO}_{2}$ and NOx, as shown in Figure 3.12. The oscillations in the instantaneous PM predictions could be significantly reduced using an infinite impulse filter at the expense of slightly retarded response. It must be noted that the negative values seen in the instantaneous PM emissions are an artifact of the measurement methodology. This was due to the condensation of water vapor and volatile organic hydrocarbons on the oscillating microbalance filter followed by re-evaporation at engine operation with high exhaust gas temperatures. The predictions for the emissions of carbon monoxide (CO) and total hydrocarbons (THC) are much less satisfactory, as shown in Figures 3.13 and 3.14. The predictions were filtered to suppress the prediction noise, especially for the THC emissions. The onset of the sharp spikes seen in $\mathrm{CO}$ emissions were predicted accurately. The large variation in the magnitude of $\mathrm{CO}$ emissions during highly transient operation results in poor prediction 
capability for the low levels of emissions seen through most of the cycle. This result was carried over to the THC emissions, which had a relatively high level of background emissions. Added to this its complex nature of hydrocarbon emissions formation (via blow-by, quenching on the chamber walls, and oxidation in the exhaust port) makes it difficult to achieve any meaningful transient predictions, as shown in Figure 3.14. The airflow prediction for the second stage of model validation was shown in Figure 3.15. The engine speed and control vectors trajectories measured over the FTP were used as inputs to the open loop simulation. Very good agreement was obtained demonstrating the capability of the $\mathrm{NN}$ to predict the turbocharger dynamics accurately.

In order to provide more precise quantification of the prediction accuracy of each $\mathrm{NN}$, several error measures were developed and used to quantify the prediction errors over the NN training procedure, parallel-series mode validation, and open loop simulation. The root mean square error, $\mathrm{e}_{1}$, was defined in terms of the total number of points, $\mathrm{N}$, the measurement vector $\mathrm{t}$, and the prediction vector, a, as follows:

$$
e_{1}=\sqrt{\frac{1}{N} \sum_{i=1}^{i=N}\left(t_{i}-a_{i}\right)^{2}}
$$

The error measure, $\mathrm{e}_{2}$, was the instantaneous relative error expressed as the value of $\mathrm{e}_{1}$ divided by the range of the predicted parameter calculated from its minimum and maximum bounds shown in Table 3.2. The integral relative error, $\mathrm{e}_{3}$, was calculated from:

$$
e_{3}=1-\frac{\sum_{i=1}^{i=N} a_{i}}{\sum_{i=1}^{i=N} t_{i}}
$$


The error in predictions of the integral brake specific emissions, $\mathrm{e}_{4}$, was of primary concern from an emissions standard regulatory point of view. This was calculated for the $\mathrm{i}^{\text {th }}$ emission in terms of $\mathrm{e}_{3, \mathrm{i}}$ and the positive power integral relative error, $\mathrm{e}_{3, \mathrm{pwr}+}$, as follows:

$$
\mathrm{e}_{4, \mathrm{i}}=1-\frac{\left(1-\mathrm{e}_{3, \mathrm{i}}\right)}{\left(1-\mathrm{e}_{3, \mathrm{pwr}+}\right)}
$$

A more detailed insight into the $\mathrm{NN}$ prediction errors could be obtained through regression analysis between the $\mathrm{NN}$ output values and the target measurements. In this respect three parameters were considered. These were the y-intercept, $\mathrm{c}$, the slope, $\mathrm{m}$, and the coefficient of determination, r2, of the best linear fit of the predictions to the measurements. For perfect fit, these take the values $0.0,1.0$, and 1.0, respectively. A non-zero intercept value indicates a persistent bias in the predictions. The closer the slope value is to unity the higher the prediction accuracy, in the mean value sense. Finally, the greater the deviation of the regression coefficient from unity, the higher the scatter in the predictions around the mean values of measurement.

The prediction errors and regression analysis of the results for the engine operation variables are shown in Table 3.3 for the training procedure, the validation test, and the open loop simulation. The instantaneous relative error, $\mathrm{e}_{2}$, in predictions of the airflow rate did not exceed $2.5 \%$, even for the parallel mode simulation. The maximum integral relative error, $\mathrm{e}_{3}$, was less than $0.25 \%$, and the regression analysis confirms the accuracy of the prediction. The instantaneous error in brake torque predictions was less than 3\%, the integral error was relatively higher ( $\sim \%)$ over the validation and open loop simulation, and the regression analysis indicates no bias, accurate mean value predictions, and low scatter. Accurate predictions for brake power 
and brake positive power were obtained with higher accuracy for the integral results $(\sim 2 \%)$ compared to torque $(\sim 6 \%)$, as shown in Table 3.3 .

The exhaust emissions prediction errors and regression analysis results are shown in Table 3.4. The errors for $\mathrm{CO}_{2}$ predictions did not exceed $3.5 \%$ on instantaneous basis, $2.5 \%$ on integral basis, and $2.1 \%$ for the integral brake specific emissions.

Table 3.3 Prediction integral error analysis for engine operating parameters

\begin{tabular}{|c|c|c|c|c|c|c|}
\hline \multicolumn{7}{|c|}{ TRAINING RESULTS } \\
\hline \multirow[t]{2}{*}{ OPERATING PARAMETERS } & \multicolumn{3}{|c|}{ error } & \multicolumn{3}{|c|}{ regression } \\
\hline & e1 & e2 (\%) & e3 (\%) & $\mathrm{c}$ & $\mathrm{m}$ & r2 \\
\hline Air Flow Rate (mg/cyc) & 150.93 & 1.89 & -0.01 & 89.58 & 0.9907 & 0.9908 \\
\hline Brake Torque (ft-lbf) & 31.54 & 2.74 & 0.00 & 6.34 & 0.9563 & 0.9563 \\
\hline Brake Power (bhp) & 9.63 & 1.93 & 0.03 & 1.33 & 0.9711 & 0.9692 \\
\hline Brake Positive Power (bhp) & 8.56 & 2.44 & 0.39 & 0.96 & 0.9768 & 0.9723 \\
\hline \multicolumn{7}{|c|}{ VALIDATION RESULTS } \\
\hline \multirow[t]{2}{*}{ OPERATING PARAMETERS } & \multicolumn{3}{|c|}{ error } & \multicolumn{3}{|c|}{ regression } \\
\hline & e1 & e2 (응 & e3 (\%) & $\mathrm{C}$ & \begin{tabular}{l|l}
$\mathrm{m}$ &
\end{tabular} & $r 2$ \\
\hline Air Flow Rate (mg/cyc) & 147.76 & 1.85 & 0.03 & 45.39 & 0.9947 & 0.9891 \\
\hline Brake Torque (ft-lbf) & 29.95 & 2.60 & 5.61 & -5.45 & 1.0117 & 0.9571 \\
\hline Brake Power (bhp) & 8.33 & 1.67 & 2.12 & -1.14 & 1.0170 & 0.9765 \\
\hline Brake Positive Power (bhp) & 7.03 & 2.01 & -0.35 & -0.27 & 1.0115 & 0.9806 \\
\hline \multicolumn{7}{|c|}{ OPEN LOOP SIMULATION RESULTS } \\
\hline \multirow[t]{2}{*}{ OPERATING PARAMETERS } & \multicolumn{3}{|c|}{ error } & \multicolumn{3}{|c|}{ regression } \\
\hline & $\mathrm{e} 1$ & e2 (응 & e3 (\%) & C & $\mathrm{m}$ & $\mathrm{r2}$ \\
\hline Air Flow Rate (mg/cyc) & 187.56 & 2.34 & -0.24 & 146.91 & 0.9863 & 0.9827 \\
\hline Brake Torque (ft-lbf) & 32.43 & 2.82 & 6.14 & -6.34 & 1.0174 & 0.9508 \\
\hline Brake Power (bhp) & 9.15 & 1.83 & 2.19 & -1.17 & 1.0172 & 0.9719 \\
\hline Brake Positive Power (bhp) & 7.33 & 2.10 & -0.77 & -0.12 & 1.0113 & 0.9789 \\
\hline
\end{tabular}

The NOx prediction errors were less than $3.1 \%$ on an instantaneous basis, $3.6 \%$ on an integral basis, and $4.4 \%$ on an integral brake specific basis. The regression analysis for both $\mathrm{CO}_{2}$ and NOx indicated high accuracy of predictions, as shown in Table 3.4. Relatively less accuracy was obtained for PM predictions with maximum instantaneous error of 5.9\%. The integral PM prediction errors were much higher $(\sim 9.5 \%)$ for the open loop simulation compared to the training and validation prediction errors $(\sim 0.3 \%)$. The PM prediction regression analysis results were not as good as those obtained for $\mathrm{CO}_{2}$ and $\mathrm{NOx}$ predictions. Though low error values were obtained for $\mathrm{CO}$ and $\mathrm{THC}$ predictions (less than 5\%), the regression analysis confirmed the 
previously obtained result that transient predictions of $\mathrm{CO}$ and $\mathrm{THC}$ emissions are not accurate, especially for low level emissions, as shown in Table 3.4.

Table 3.4 Prediction error analysis for exhaust emissions

\begin{tabular}{|c|c|c|c|c|c|c|c|c|}
\hline \multicolumn{9}{|c|}{ TRAINING RESULTS } \\
\hline \multirow[t]{2}{*}{ EMISSIONS $(g / h)$} & \multicolumn{5}{|c|}{ Error } & \multicolumn{3}{|c|}{ Regression } \\
\hline & e1 & e2 & $(\%)$ & e3 (\%) & e4 (\%) & C & $\mathrm{m}$ & r2 \\
\hline Carbon Dioxide & 3841.60 & & 3.34 & -0.02 & -0.42 & 621.84 & 0.9806 & 0.9807 \\
\hline Oxides of Nitrogen & 29.42 & & 2.94 & 0.00 & -0.39 & 5.12 & 0.9811 & 0.9812 \\
\hline Particulate matter & 5.25 & & 5.25 & -0.09 & -0.48 & 0.48 & 0.9025 & 0.9025 \\
\hline Carbon Monoxide & 70.42 & & 3.52 & 2.98 & 2.60 & 16.82 & 0.8104 & 0.8210 \\
\hline Total Hydrocarbons & 3.12 & & 3.12 & -0.16 & -0.55 & 1.42 & 0.8408 & 0.8422 \\
\hline \multicolumn{9}{|c|}{ VALIDATION RESULTS } \\
\hline \multirow[t]{2}{*}{ EMISSIONS $(g / h)$} & \multicolumn{5}{|c|}{ Error } & \multicolumn{3}{|c|}{ Regression } \\
\hline & e1 & e2 & $\left(\frac{\circ}{0}\right)$ & e3 (\%) & e4 (\%) & $\mathrm{C}$ & $\mathrm{m}$ & $\mathrm{r} 2$ \\
\hline Carbon Dioxide & 3788.97 & & 3.29 & -2.43 & -2.07 & 351.59 & 1.0081 & 0.9802 \\
\hline Oxides of Nitrogen & 29.17 & & 2.92 & 3.05 & 3.39 & -0.19 & 0.9705 & 0.9839 \\
\hline Particulate matter & 5.84 & & 5.84 & -0.25 & 0.10 & 0.58 & 0.8075 & 0.8863 \\
\hline Carbon Monoxide & 46.58 & & 2.33 & -3.27 & -2.90 & 6.67 & 0.9056 & 0.7154 \\
\hline Total Hydrocarbons & 2.37 & & 2.37 & -2.51 & -2.15 & 0.14 & 1.0052 & 0.3022 \\
\hline \multicolumn{9}{|c|}{ OPEN LOOP SIMULATION RESULTS } \\
\hline \multirow[t]{2}{*}{ EMISSIONS $(g / h)$} & \multicolumn{5}{|c|}{ Error } & \multicolumn{3}{|c|}{ Regression } \\
\hline & e1 & e2 & $\left(\frac{\circ}{0}\right)$ & e3 (\%) & e4 (\%) & C & $\mathrm{m}$ & r2 \\
\hline Carbon Dioxide & 3792.89 & & 3.30 & -1.82 & -1.04 & -48.46 & 1.0204 & 0.9807 \\
\hline Oxides of Nitrogen & 30.09 & & 3.01 & 3.58 & 4.32 & -2.96 & 0.9795 & 0.9829 \\
\hline Particulate matter & 5.85 & & 5.85 & 8.76 & 9.46 & 0.26 & 0.8232 & 0.8840 \\
\hline Carbon Monoxide & 43.81 & & 2.19 & -4.47 & -3.67 & 12.40 & 0.8082 & 0.7160 \\
\hline Total Hydrocarbons & 2.36 & & 2.36 & -1.34 & -0.56 & -0.03 & 1.0175 & 0.3231 \\
\hline
\end{tabular}

Table 3.5 EPA emissions standards for HD diesel engines (g/bhp-h)

\begin{tabular}{|r|r|r|r|r|}
\hline Model Year & \multicolumn{1}{|c|}{ THC } & \multicolumn{1}{|c|}{ CO } & NOx & PM \\
\hline \multicolumn{2}{|c|}{ Heavy Duty Diesel Truck Engines } \\
\hline 1990 & 1.3 & 15.5 & 6.0 & 0.6 \\
\hline 1991 & 1.3 & 15.5 & 5.0 & 0.3 \\
\hline 1994 & 1.3 & 15.5 & 5.0 & 0.1 \\
\hline 1998 & 1.3 & 15.5 & 4.0 & 0.1 \\
\hline \multicolumn{7}{|c|}{ Urban Bus Engines } \\
\hline 1991 & 1.3 & 15.5 & 5.0 & 0.3 \\
\hline 1993 & 1.3 & 15.5 & 5.0 & 0.1 \\
\hline 1994 & 1.3 & 15.5 & 5.0 & 0.1 \\
\hline 1996 & 1.3 & 15.5 & 5.0 & 0.1 \\
\hline 1998 & 1.3 & 15.5 & 4.0 & 0.1 \\
\hline \multicolumn{7}{|c|}{ Test Engine: Navistar T444E } \\
\hline 1994 & 0.209 & 1.731 & 5.640 & 0.103 \\
\hline
\end{tabular}

In order to put the above emissions predictions in perspective, a brief discussion of exhaust emissions and their regulation standards for modern DI diesel engines is required. In general, the major regulated pollutants emitted from a direct injection compression ignition engine are oxides of nitrogen (NOx) and particulate matter (PM). The concentration of NOx emissions are 
comparable to those from SI engines while the hydrocarbon (HC) emissions are lower by about a factor of 5 and carbon monoxide (CO) emissions are negligible (Heywood, 1988; Kawakami et al., 1994). A CI DI engine optimized for minimum fuel consumption would emit low PM and excessively high NOx. The PM emissions would be low by virtue of the efficient oxidation because of the high in-cylinder gas temperature and an extended residence time due to an advanced start of injection. The prolonged combustion event however results in higher temperatures and higher NOx emissions. The EPA emission standards for HD diesel engines are shown in Table 3.5. Also shown are the brake specific emissions of the test engine over the FTP cycle at normal operating conditions (hot operation). The CO and THC emissions are well below the regulatory limit. On the other hand, the emissions of NOx and PM are in the vicinity of the regulatory levels. This result amplifies the need for accurate predictions of NOx and PM and deemphasizes the inaccuracies in the predictions of $\mathrm{CO}$ and $\mathrm{THC}$ emissions. 


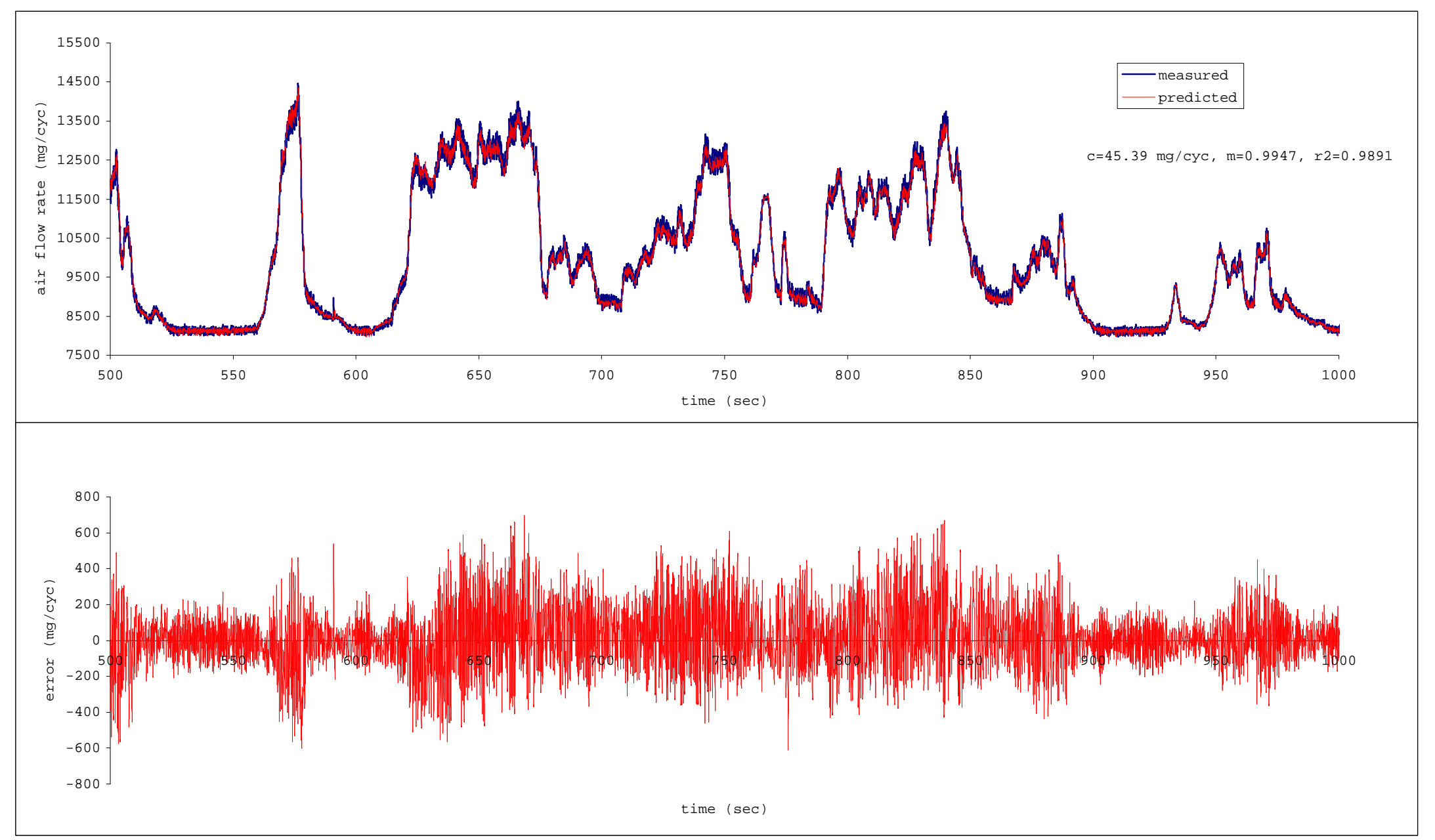

Figure 3.8 Measurement and series-parallel mode prediction of air flow rate over the FTP cycle 


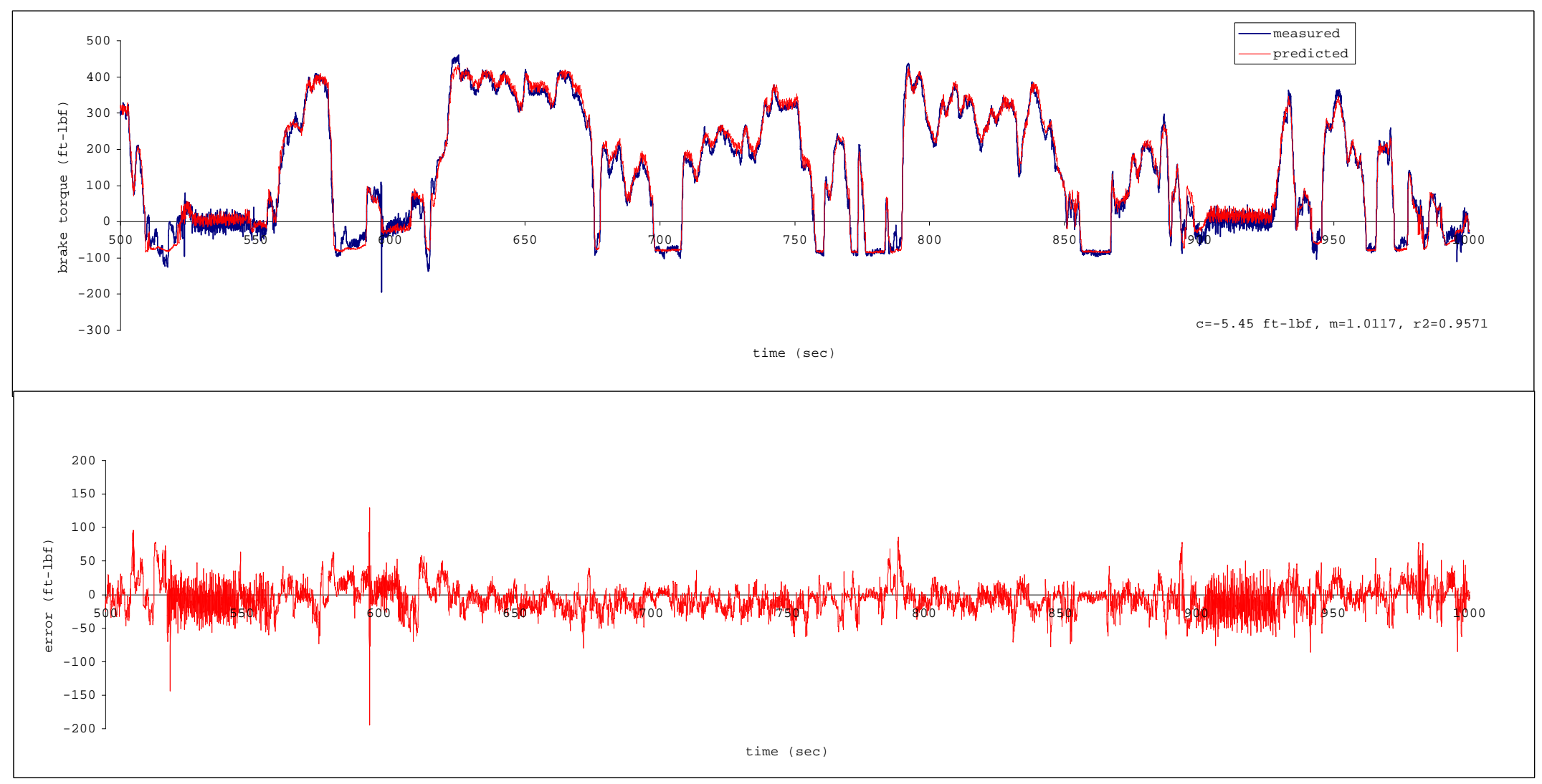

Figure 3.9 Measured and predicted engine brake torque over the FTP cycle

74 


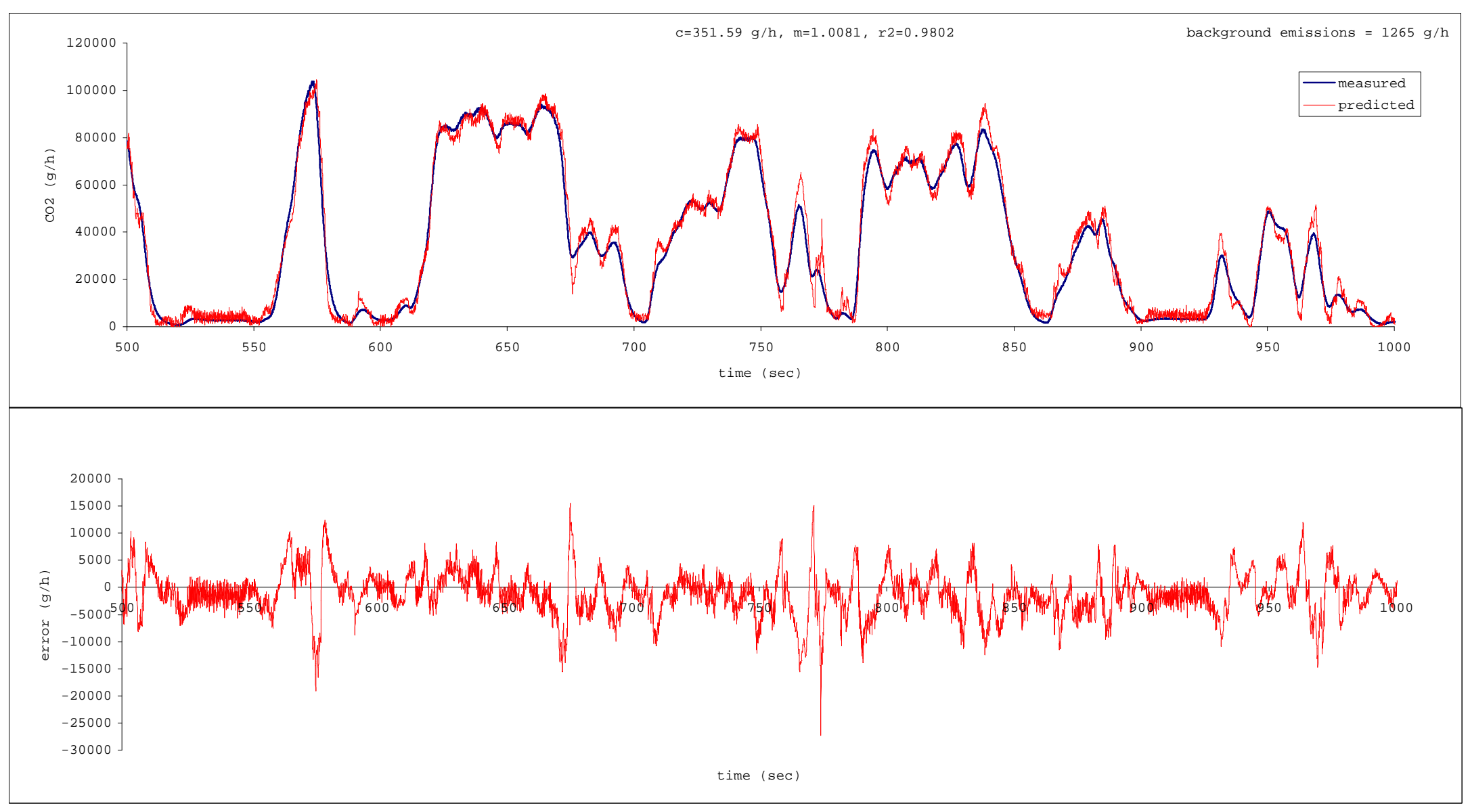

Figure 3.10 Measured and predicted CO2 emissions over the FTP cycle

75 


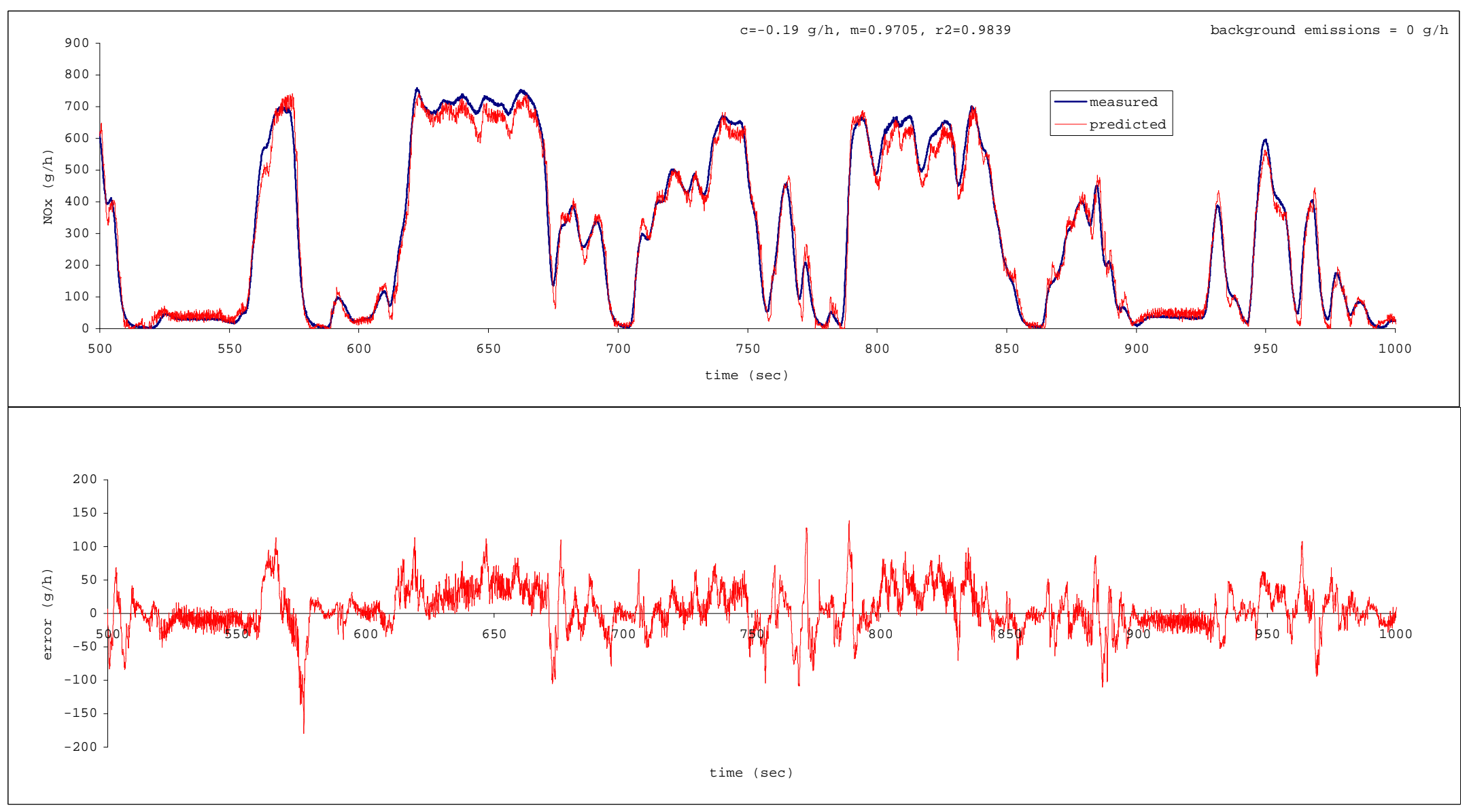

Figure 3.11 Measured and predicted NOr emissions over the FTP cycle

76 


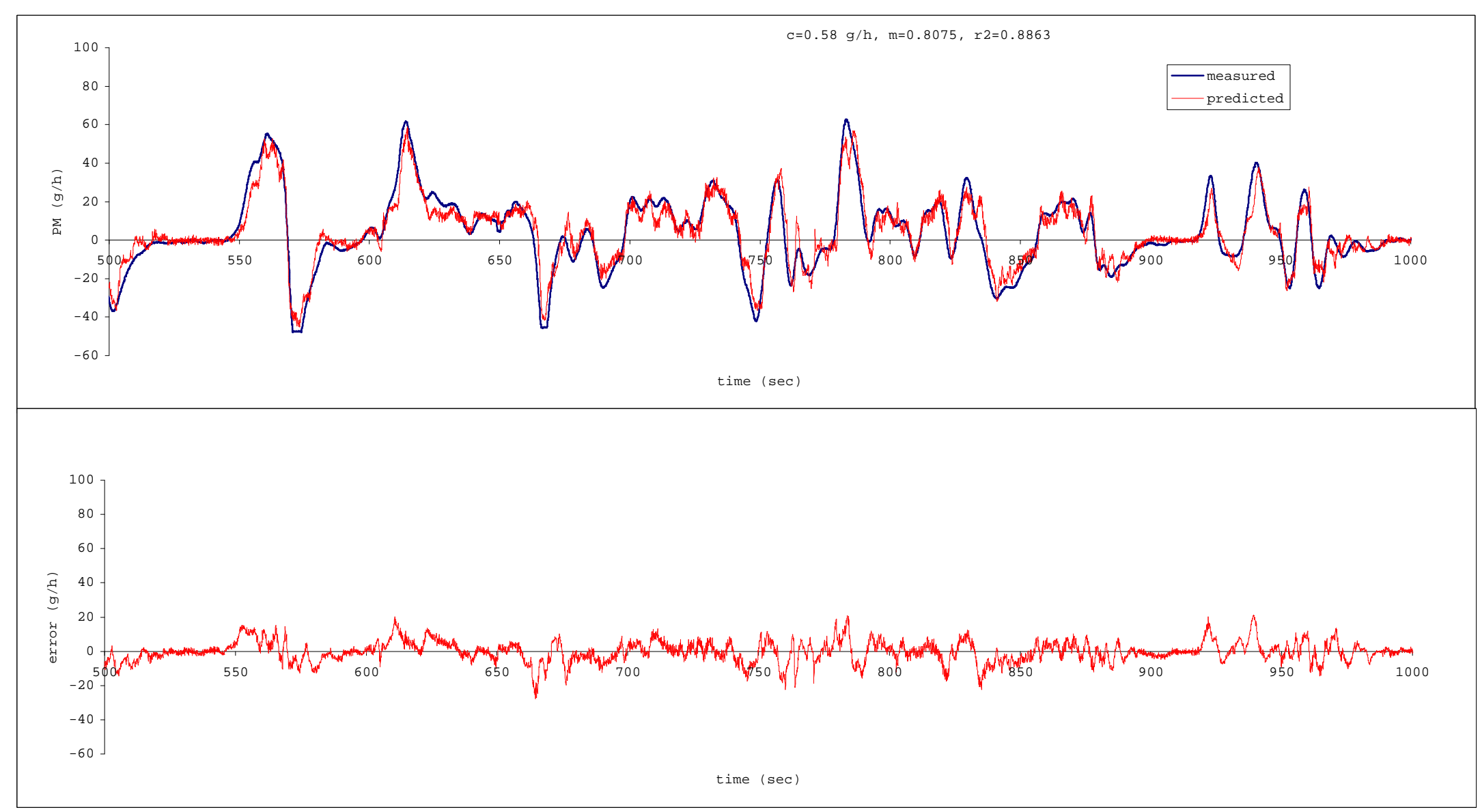

Figure 3.12 Measured and predicted PM emissions over the FTP cycle 


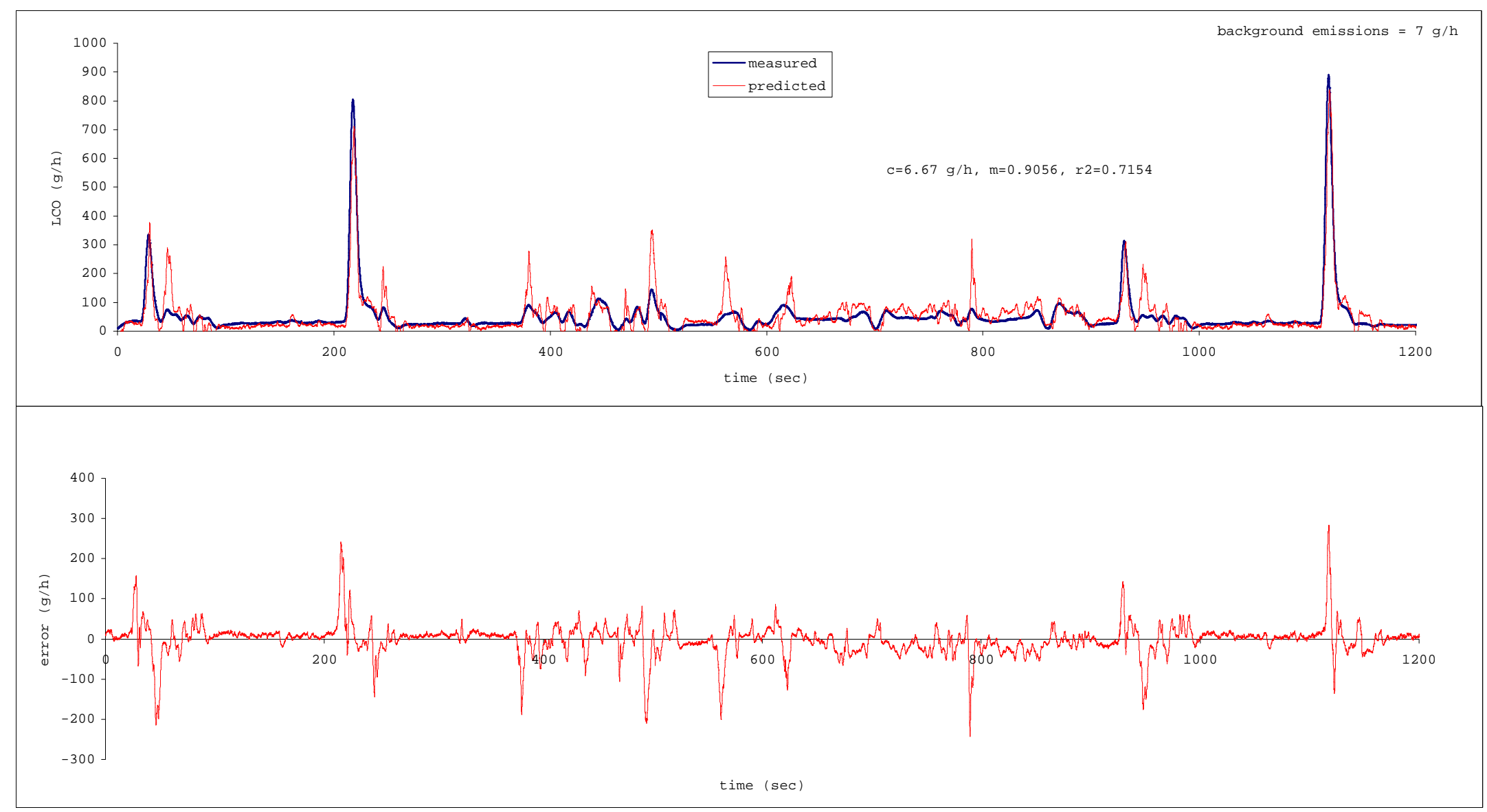

Figure 3.13 Measured and predicted CO emissions over the FTP cycle 


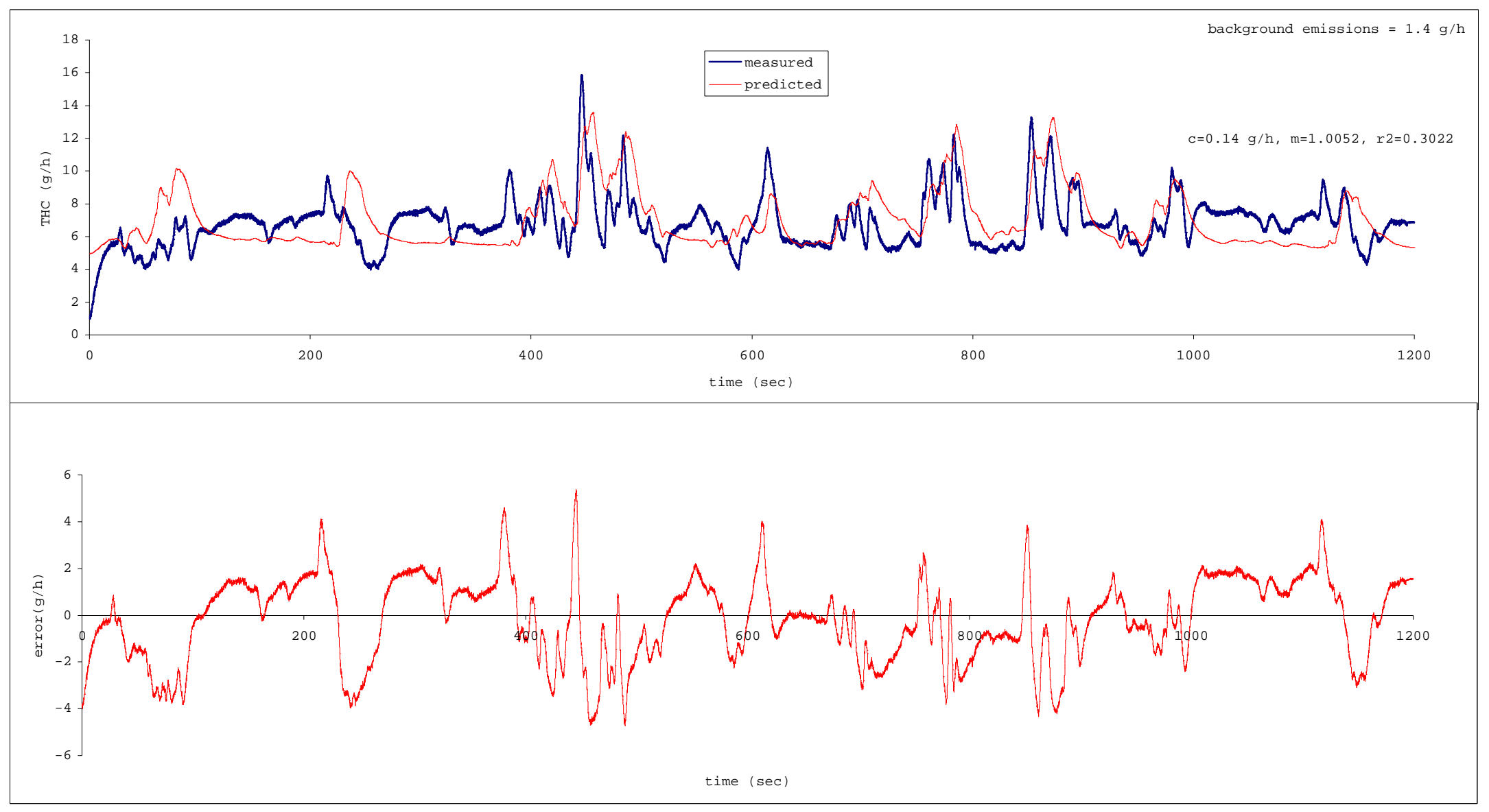

Figure 3.14 Measured and predicted THC emissions over the FTP cycle

79 


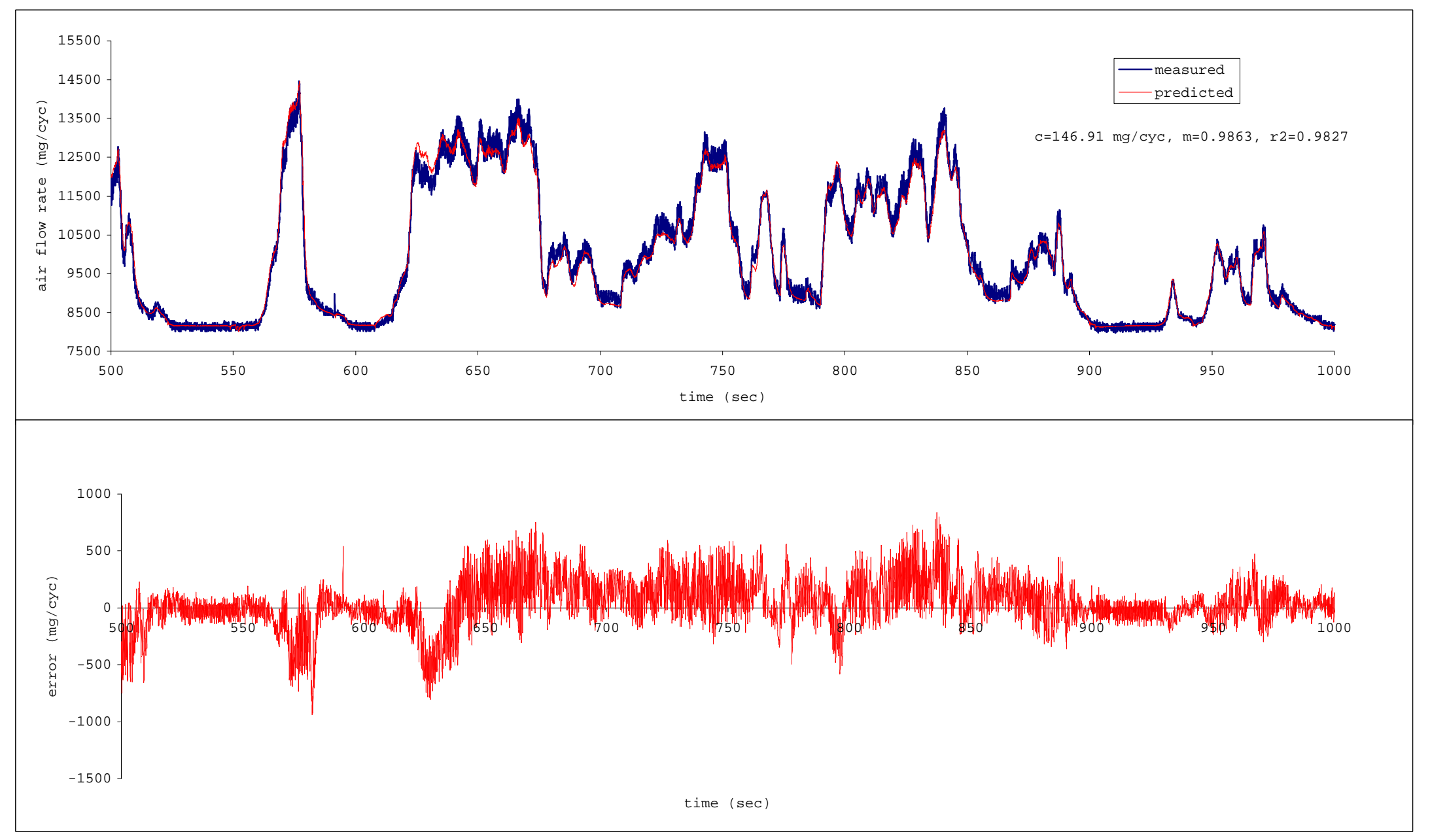

Figure 3.15 Measurement and parallel mode prediction of air flow rate over the FTP cycle 


\section{CHAPTER 4}

\section{Neural Network-based Engine Control}

\subsection{Overview}

In the current investigation, the DC dynamometer controller was used to track the prescribed speed schedule at a rate of $10 \mathrm{~Hz}$, as was previously outlined in Section 2.1. The engine torque control was achieved using a combination of feedforward and closed-loop proportional-plusintegral (PI) actuation of the drive-by-wire accelerator position signal (APS). For a prescribed engine speed, the APS was initially set at its zero-torque value and subsequently corrected at a rate of $10 \mathrm{~Hz}$. Since all test cycles were prescribed at one-second interval, linear interpolation was used to achieve continuous transition between scheduled values. The tracking performance

of speed and torque over the conducted test schedules is shown in Table 4.1. Excellent tracking of the prescribed speed was achieved for all the conducted test schedules, as shown in Table 4.1a (c should be zero, $m$ unity, and $r 2$ unity). This is attributed to the fact that the response time of the dynamometer speed control was approximately one order of magnitude less than that for the engine torque control. Much less satisfactory tracking performance was achieved for the scheduled torque values, as shown in Table 4.1b. For the purpose of engine certification, negative torque values and unattainable values at wide-open-throttle are eliminated from the regression analysis (40CFR Part 86 Subpart N, 1996). The positive torque tracking performance was better, especially for the FTP, as shown in Table 4.1c, than the total torque tracking performance shown in Table 4.1b. The actual and desired engine speed and torque time traces are shown in Figures 4.1 and 4.2 for the FTP and random test cycle A, respectively.

The EPA validation criteria for HD CI engines tested over the FTP test cycle are shown in Table 4.2. Tighter tolerances are specified for speed control than for torque control. For all the 
test cycles conducted, the engine speed validation criteria were met with significant margins. On the other hand, test cycles D, G, and $\mathrm{H}$ failed to meet the coefficient of determination (r2) validation criteria for engine torque, mainly due to their aggressive nature. Test cycle B failed to meet the y-intercept (c) validation criteria indicating a persistent bias in the achieved torque values. The difficulty in achieving tight tracking of the prescribed torque schedule was mainly attributed to two factors. The first factor was the highly non-linear dependence of fueling on APS signal because of the all-speed governor, which was software-based for the test engine. This effect could be eliminated if direct control of the fueling rate were employed thus by-passing the governor. The second factor was the turbocharger transient response especially under hard acceleration where a smoke-limiting strategy was invoked. Both of these problems could have been effectively remedied if a predictive optimal control algorithm were employed rather than the implemented PI control algorithm, which was heuristically tuned for a satisfactory global performance.

The objective of the work described in this chapter is twofold. Firstly, to develop and validate through simulation a neural network-based torque controller for the purpose of tracking a prespecified arbitrary schedule. The cases of non-governed as well as governed engine operation are considered. Secondly, to develop and validate through simulation a neural network based allspeed governor. Several NNs are developed for the purpose of generating commanded control parameters, which include fuel flow rate, injection timing, and injection pressure. The controller NNs are trained offline and individually validated against measurement. Each controller is then integrated with the previously developed mean value engine model and adapted online to allow the prescribed torque schedule to be tracked. Closed-loop performance is examined through simulation over several arbitrarily test cycles. The performance criteria considered are not 
limited to the tracking ability of each controller configuration but also to the reduction in transient exhaust gas emissions over the test cycles.

Table 4.1a Tracking error analysis for measured engine speed

\begin{tabular}{|l|r|r|r|r|r|r|}
\hline \multirow{2}{*}{ Cycle } & \multicolumn{3}{|c|}{ Error } & \multicolumn{3}{|c|}{ Regression } \\
\cline { 2 - 8 } & e1 $($ rpm) & e2 (\%) & e3 $(\%)$ & \multicolumn{1}{c|}{ c } & \multicolumn{1}{c|}{ m } & \multicolumn{1}{c|}{ 2 } \\
\hline FTP & 13.76 & 0.57 & -0.24 & 0.0991 & 1.0024 & 0.9996 \\
\hline ARB A & 14.43 & 0.60 & -0.06 & -4.1367 & 1.0033 & 0.9995 \\
\hline ARB B & 16.28 & 0.68 & -0.17 & 0.1144 & 1.0016 & 0.9994 \\
\hline ARB C & 15.72 & 0.66 & -0.15 & -2.7698 & 1.0032 & 0.9994 \\
\hline ARB D & 24.91 & 1.04 & -0.11 & -3.5612 & 1.0034 & 0.9986 \\
\hline ARB E & 14.84 & 0.62 & -0.14 & -4.8998 & 1.0045 & 0.9995 \\
\hline ARB F & 20.25 & 0.84 & -0.20 & 2.2437 & 1.0007 & 0.9991 \\
\hline ARB G & 60.57 & 2.52 & 0.03 & -3.2177 & 1.0017 & 0.9917 \\
\hline ARB H & 20.44 & 0.85 & -0.21 & 3.0347 & 1.0003 & 0.9991 \\
\hline FTP & 15.21 & 0.63 & -0.24 & -0.0876 & 1.0025 & 0.9995 \\
\hline
\end{tabular}

Table 4.1b Tracking error analysis for measured engine torque

\begin{tabular}{|l|r|r|r|r|r|r|}
\hline \multirow{2}{*}{ Cycle } & \multicolumn{3}{|c|}{ Error } & \multicolumn{3}{|c|}{ Regression } \\
\cline { 2 - 7 } & e1 & e2 (\%) & e3 $(\%)$ & \multicolumn{1}{c|}{ c } & \multicolumn{1}{c|}{ m } & \multicolumn{1}{c|}{ 2 } \\
\hline FTP & 67.56 & 5.87 & -12.35 & 23.1316 & 0.8045 & 0.8151 \\
\hline ARB A & 44.38 & 3.86 & -8.71 & 11.5159 & 0.9463 & 0.9291 \\
\hline ARB B & 41.91 & 3.64 & -3.70 & 10.7823 & 0.9580 & 0.9264 \\
\hline ARB C & 42.01 & 3.65 & -0.23 & 7.1041 & 0.9535 & 0.9219 \\
\hline ARB D & 52.65 & 4.58 & 3.86 & 3.4091 & 0.9404 & 0.8509 \\
\hline ARB E & 34.64 & 3.01 & 1.97 & -0.6641 & 0.9840 & 0.9337 \\
\hline ARB F & 43.78 & 3.81 & 1.84 & 1.5449 & 0.9730 & 0.9020 \\
\hline ARB G & 54.63 & 4.75 & 2.97 & 3.2209 & 0.9517 & 0.8511 \\
\hline ARB H & 62.12 & 5.40 & 1.07 & 7.0849 & 0.9439 & 0.8412 \\
\hline FTP & 53.67 & 4.67 & -11.66 & 20.3106 & 0.8365 & 0.8870 \\
\hline
\end{tabular}

Table 4.1c Tracking error analysis for measured engine positive torque

\begin{tabular}{|l|r|r|r|r|r|r|}
\hline \multirow{2}{*}{ Cycle } & \multicolumn{4}{|c|}{ Error } & \multicolumn{3}{|c|}{ Regression } \\
\cline { 2 - 7 } & e1 & e2 $\left(\frac{\circ}{0}\right)$ & e3 $\left(\frac{\circ}{\circ}\right)$ & \multicolumn{1}{c|}{ c } & \multicolumn{1}{c|}{ m } & r2 \\
\hline FTP & 46.95 & 6.26 & 8.69 & -5.1970 & 0.9590 & 0.8913 \\
\hline ARB A & 41.98 & 5.60 & -0.91 & 7.1949 & 0.9668 & 0.9162 \\
\hline ARB B & 43.58 & 5.81 & -3.05 & 15.8337 & 0.9388 & 0.9067 \\
\hline ARB C & 39.19 & 5.23 & 1.72 & 1.6792 & 0.9738 & 0.9188 \\
\hline ARB D & 52.65 & 7.02 & 3.86 & 3.4091 & 0.9404 & 0.8509 \\
\hline ARB E & 34.64 & 4.62 & 1.97 & -0.6641 & 0.9840 & 0.9337 \\
\hline ARB F & 43.78 & 5.84 & 1.84 & 1.5449 & 0.9730 & 0.9020 \\
\hline ARB G & 54.63 & 7.28 & 2.97 & 3.2209 & 0.9517 & 0.8511 \\
\hline ARB H & 59.16 & 7.89 & 1.62 & 8.9646 & 0.9369 & 0.8255 \\
\hline FTP & 35.64 & 4.75 & 6.79 & -4.7884 & 0.9743 & 0.9361 \\
\hline
\end{tabular}

Table 4.2 EPA validation criteria for the FTP test at normal operation

\begin{tabular}{|l|r|r|r|c|c|}
\hline \multirow{2}{*}{ Parameter } & \multicolumn{2}{|c|}{ Error } & \multicolumn{3}{c|}{ Regression } \\
\cline { 2 - 6 } & \multicolumn{1}{|c|}{ e1 } & e2 (\%) & C & m & r2 \\
\hline Speed (rpm) & 100.00 & 5.26 & \pm 50 & $0.97-1.03$ & $\geq 0.97$ \\
\hline Torque (ft-lbf) & 63.00 & 8.40 & \pm 15 & $0.83-1.03$ & $\geq 0.88$ \\
\hline
\end{tabular}




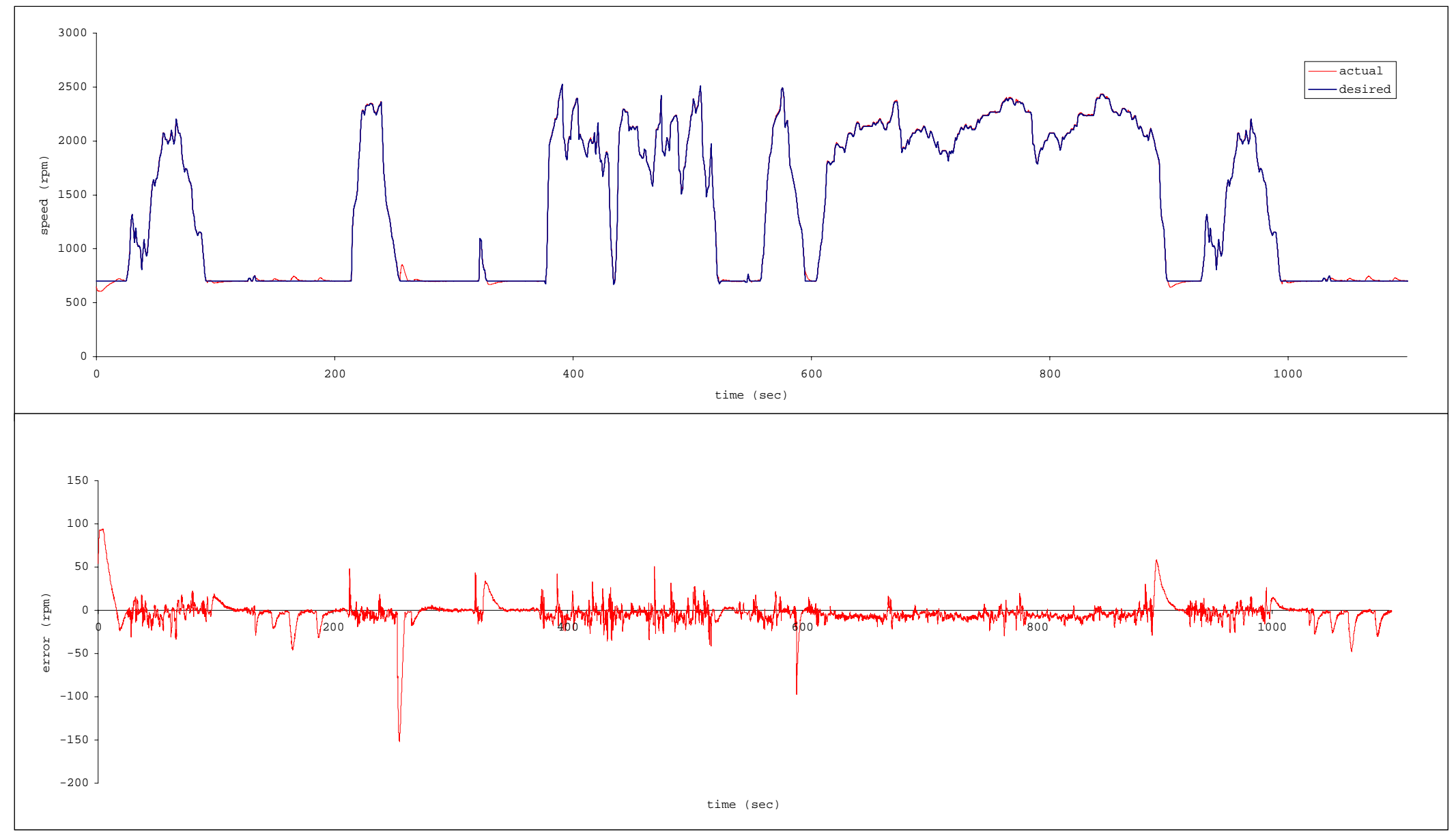

Figure 4.1a Speed history for desired and actual values over the FTP cycle 


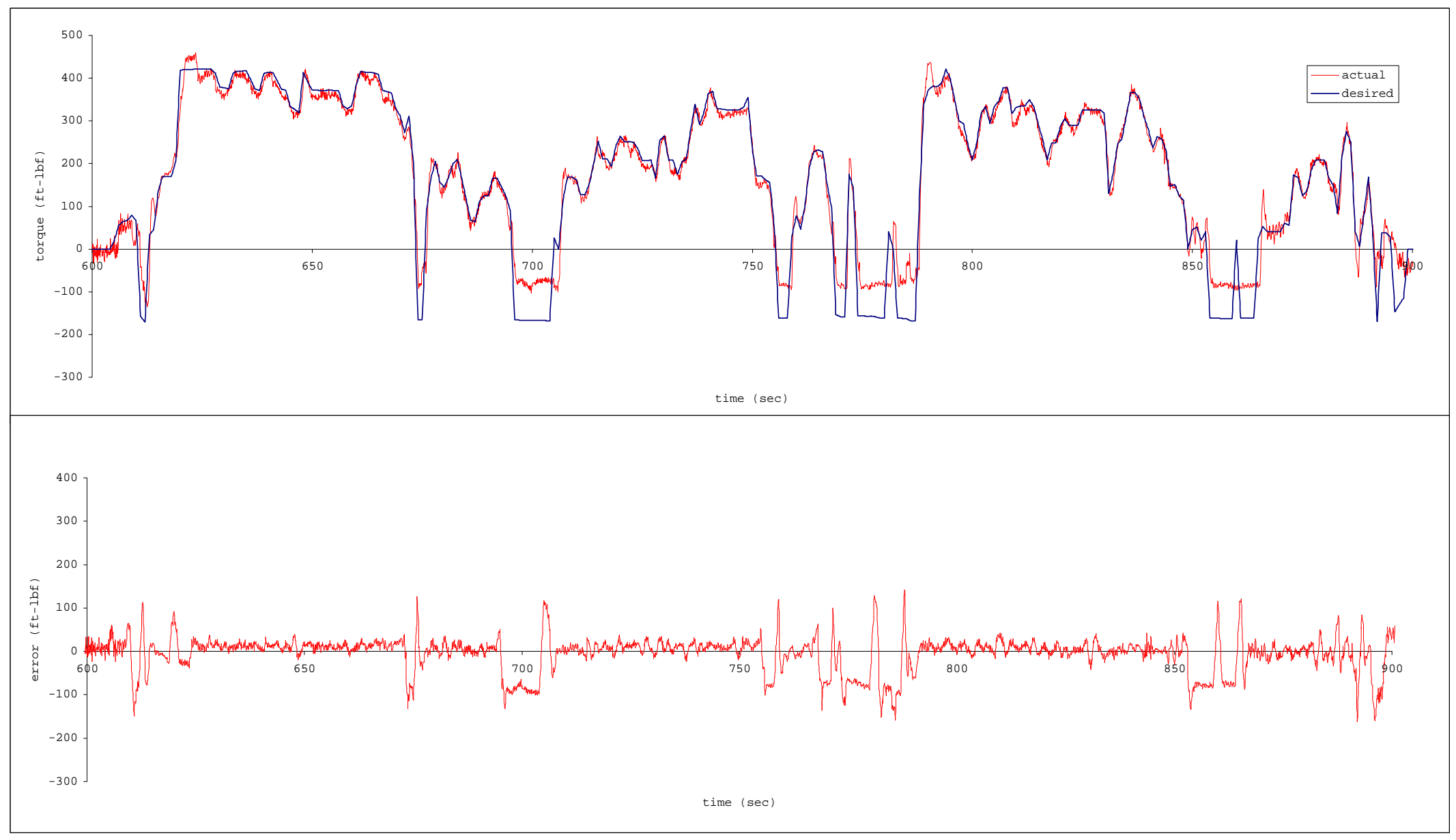

Figure 4.1b Torque history for desired and actual values over the FTP cycle 


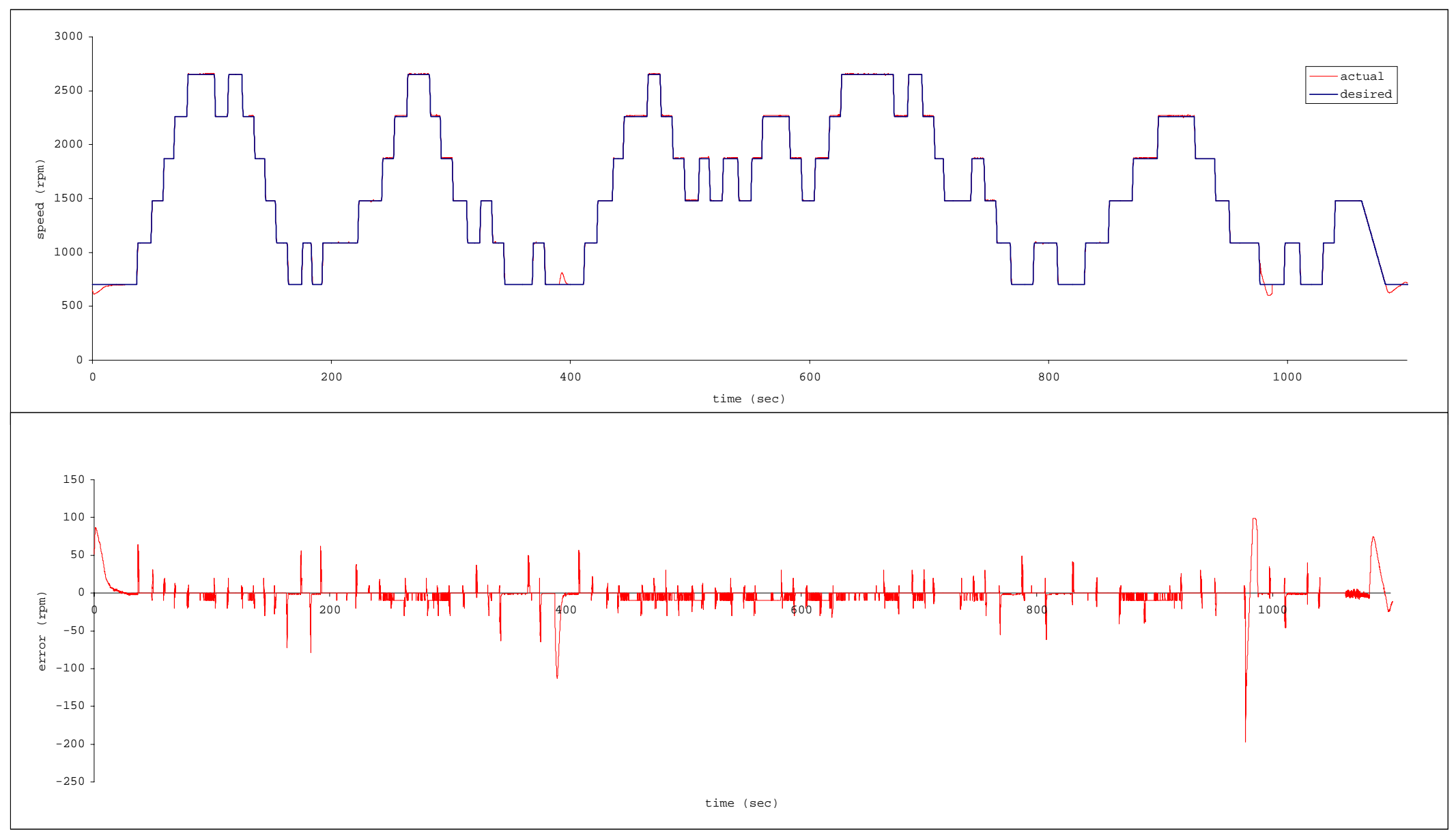

Figure 4.2a Speed history for desired and actual values over arbitrary cycle A 


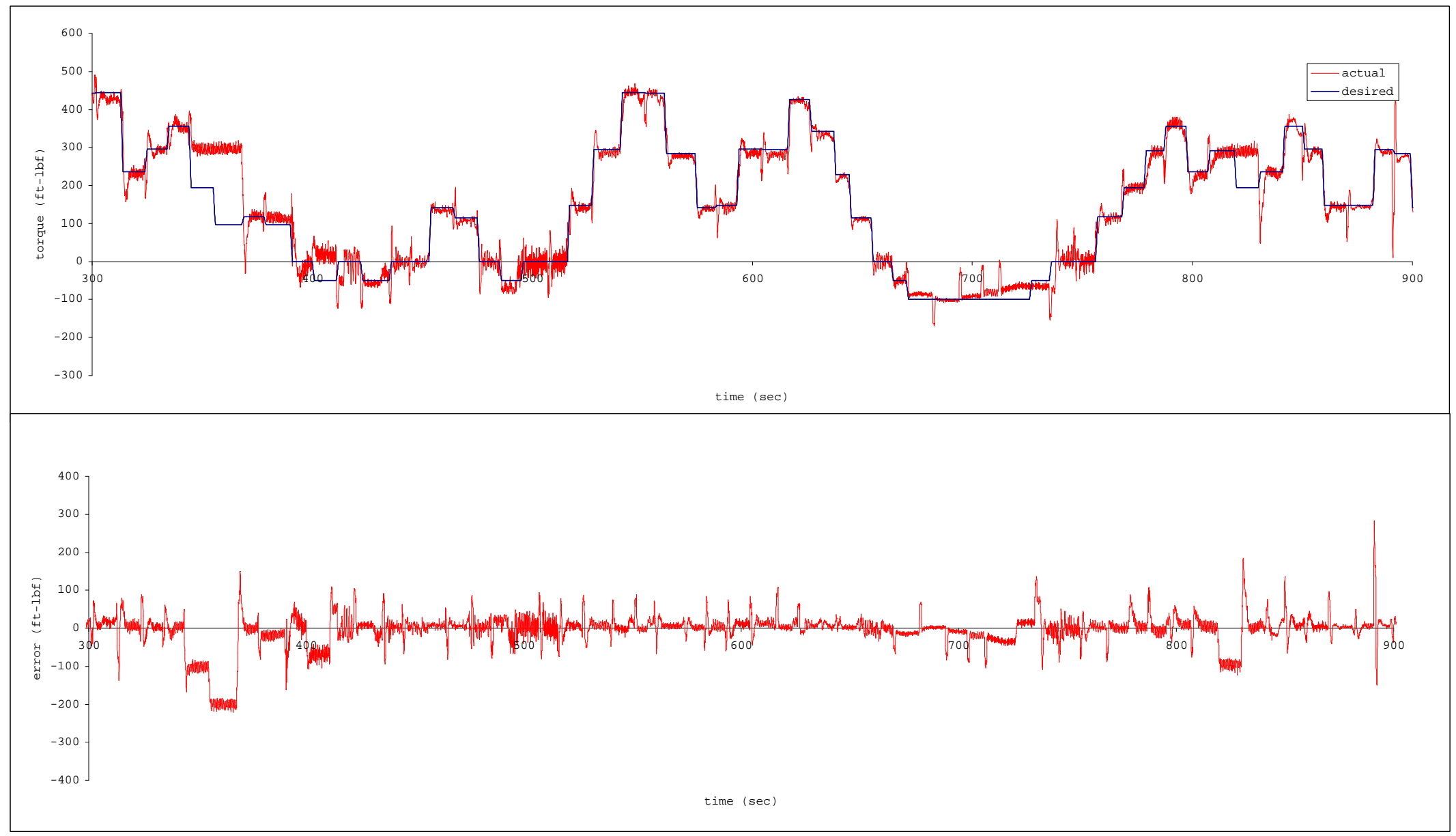

Figure 4.2b Torque history for desired and actual values over arbitrary cycle A 


\subsection{Engine Torque Controller Development and Validation}

As mentioned earlier, eliminating the speed governor would greatly facilitate tight tracking of any prescribed torque schedule by directly commanding the fuel flow rate. In the following two sections, a torque controller is firstly developed and validated in the absence of a governor. Secondly a governor, which employs fueling proportional to the APS signal, is integrated into the torque control loop.

\subsubsection{Non-Governed Engine Operation}

Different subsystems, which constitute the closed-loop mean value engine model, are shown in Figure 4.3. The input data to the closed-loop simulation are the actual engine speed trajectory and the desired brake engine torque schedule. A neural network-based controller was used to track the reference torque schedule where fuel flow rate was the control parameter. The controller's input vector consisted of current engine state vector, the torque desired at the next time step, and a preview of the engine speed at the next time increment. The output control vector consisted of the commanded fuel flow rate, injection timing, and injection pressure. The error between the desired and predicted engine torque was back-propagated through the controller neural network and used to adapt the controller NN parameters while the engine $\mathrm{NN}$ model parameters were kept frozen at the values acquired through off-line training.

In the true sense, the torque controller was a multi-input single-output system. This was due to the fact that the only control vector adjusted dynamically in a closed-loop fashion was the fuel flow rate. On the other hand, injection timing and pressure were adjusted in a feedforward sense using the original stock controller lookup tables, resident in the Ford EEC IV for this engine. 


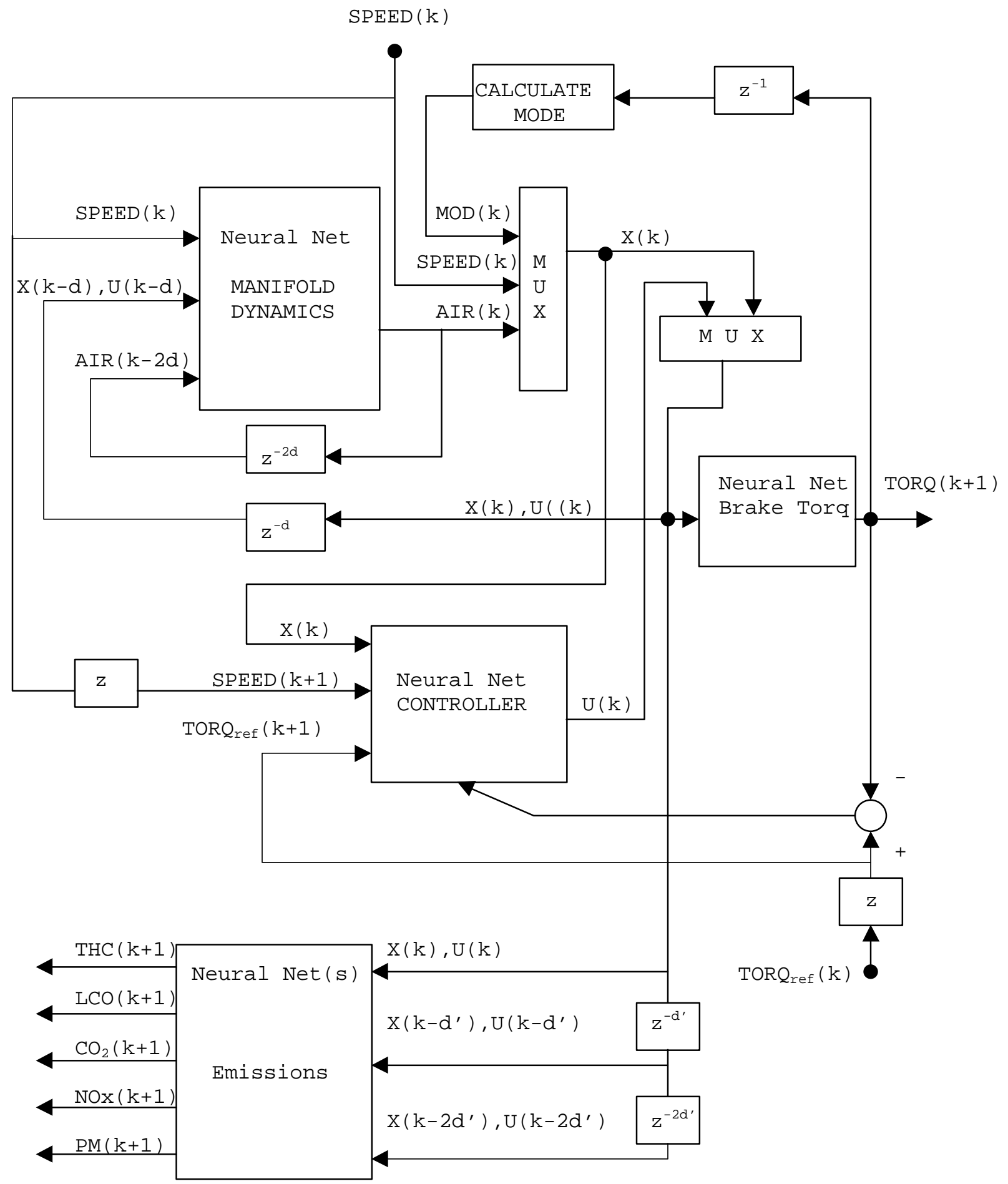

Figure 4.3 Block diagram of the closed-loop torque control 
The reason for retaining the original engine calibration was to guarantee the integrity of the model predictions. This was due to the fact that the only engine control parameter that was exercised during the engine testing was fueling via actuation of the drive-by-wire accelerator. Injection timing and pressure were merely looked up as functions of the engine operating parameters. A heuristic procedure was employed in order to arrive at original calibration lookup tables for injection timing and injection control pressure and to identify their correct functional dependencies, which was a non-trivial task since the engine calibration lookup tables and their functional dependencies were proprietary information.

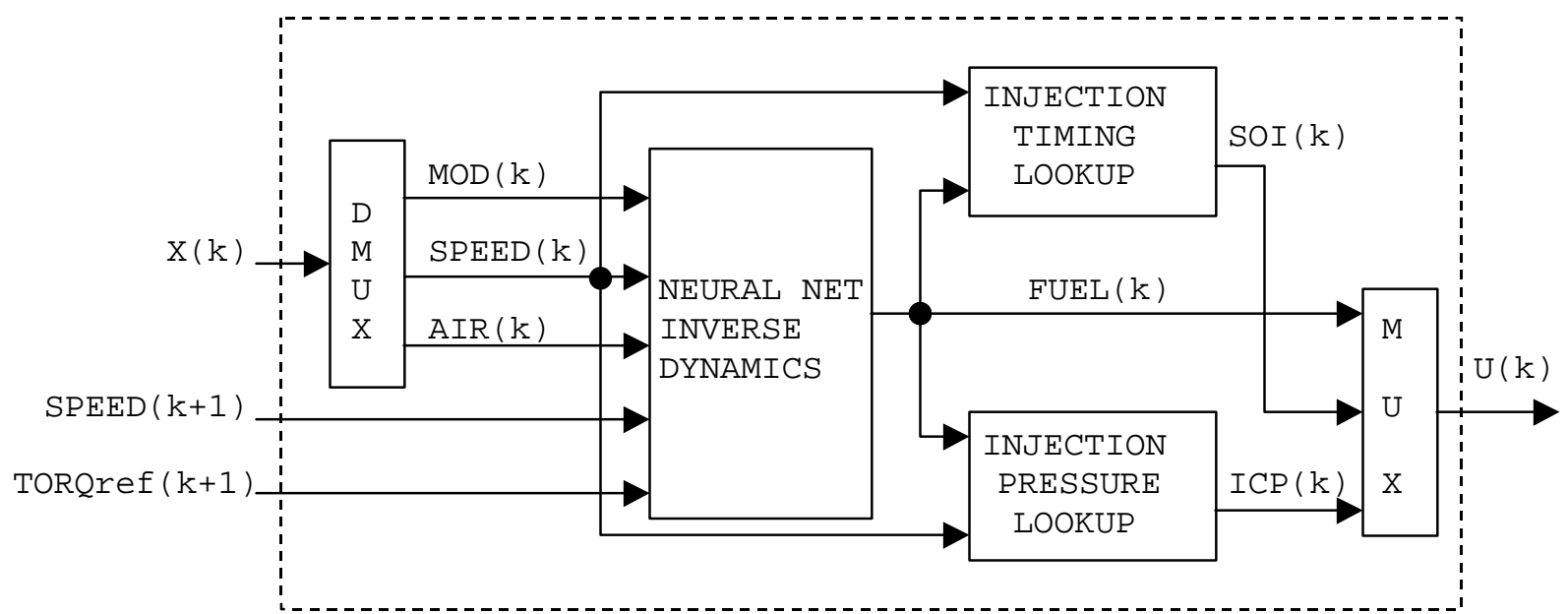

Figure 4.4 Schematic of the torque controller for non-governed operation

The details of the used controller are shown in the Figure 4.4. The fuel flow rate was firstly determined using an inverse dynamics NN. Secondly, the injection timing and pressure were looked up using engine speed and the commanded fuel flow rate. Since the engine was operated at normal conditions throughout the current study, no corrections in timing or pressure were necessary for cold operation or altitude variations. Initially, the reduction program was used to generate the necessary lookup tables for injection timing and pressure. The correct functional dependency was secured by checking the standard deviation of each entry in the generated tables 
to be below an acceptable tolerance, as shown in Figures 4.5 and 4.6 when tested against the actual values measured. Further, the generated tables were used to check the interpolated injection timing and pressure against their measured counterparts over a test cycle not included in generating the respective lookup table.

Since the above procedure mimiced to a great extent the NN approach used in the previous chapter, it was decided to model the generated lookup tables using two NNs for injection timing and injection pressure. The advantage of this approach was twofold. Firstly, any extra dependency could be easily accommodated simply because of the multivariable capability inherent in the neural network representation. In this manner stacked two-dimensional lookup tables with successive corrections (e.g. temperature and altitude corrections) could be augmented into a hyperdimensional neural network-based representation. Secondly, it allowed for a seamless integration of the injection timing and injection pressure NNs with the inverse dynamics torque controller NN. This was clearly demonstrated when adapting the controller NN online where one needed to evaluate the sensitivities of the torque tracking error to the parameters of the injection timing and injection pressure look up NNs.

In order to guarantee the initial stability of the closed-loop performance, the NN controller was trained offline using the actual torque values as a desired input and the measured fuel flow rate as a target value used to train the controller NN parameters. This a priori knowledge acquired by the controller NN, through off-line training, was proven to be very useful in avoiding the classical local minimum problems associated with the back-propagation algorithm when used for online adaptation. For simulated on-line control, the weights and biases acquired through off-line training were used to initialize the controller NN parameters, which were later adapted online. 
In a procedure similar to that described in the previous chapter, the controller NNs (injection timing, injection pressure, and fuel flow rate) were trained offline in a batch mode using the Levenberg-Marquardt algorithm in the MATLAB environment. The arbitrary cycle data, A through $\mathrm{H}$, were used in the training procedure and the FTP cycle data was used to validate the trained NN generalization capability. The basic NN architecture used was a single hidden layer with non-linear activation in both the hidden and the output layers. This guaranteed bounded control signals within the experimentally determined ranges shown in Table 3.2. The NN architectures used are shown in Table 4.3.

Table 4.3 Torque controller neural network architectures

\begin{tabular}{|l|r|r|r|}
\hline & \multicolumn{3}{|c|}{ Number of Nodes } \\
\cline { 2 - 4 } & Input & Hidden & Output \\
\hline Injection timing & 2 & 8 & 1 \\
\hline Injection pressure & 2 & 8 & 1 \\
\hline Inverse dynamics & 5 & 4 & 1 \\
\hline
\end{tabular}

The measured and estimated time-histories of injection timing, injection pressure, and fuel mass flow rate over the validation test cycle are shown in Figures 4.7, 4.8, and 4.9, respectively. The agreement between measured and estimated values for the timing and pressure was very satisfactory indicating that their appropriate dependencies have been correctly identified. This result was further confirmed through the error analysis results, shown in Table 4.4. The inverse dynamics NN predictions were in good agreement with the measured flow rate of the fuel injected, as shown in Figure 4.9 and Table 4.4. The error was highest at sharp transients and idle operation. These errors were acceptable since the fine tuning of injected fuel mass would be achieved later through online adaptation.

In order to adapt the torque controller $\mathrm{NN}$, the error between the predicted and the desired values had to be back-propagated through the torque predictor $\mathrm{NN}$ and injection timing and pressure NNs. This was achieved by combining the four NNs into one net, as shown in Figure 4.10. The 
integral network consisted of eight layers, two for each of the four NNs, which were not fully connected. All layers were nonlinear except for the output layer in the torque predictor NN, which employed linear activation. This integral NN was externally connected to the forward dynamics $\mathrm{NN}$ and the emissions prediction NNs in order to form the closed-loop mean value model, shown in Figure 4.3. The MATLAB neural network toolbox was used to conduct the closed-loop simulation and the standard back propagation algorithm, described in section 3.3.2, was used to adapt the controller NN. The parameters of all the other NNs were frozen at their values acquired through offline training. The closed-loop simulation was conducted for pulsed torque at constant engine speed cycles as well as the test cycles shown in Table 2.2b. For comparison purposes, the simulation was also conducted for a PI torque controller operating on the normalized signals. The PI controller gains were heuristically determined to achieve satisfactory global performance. It is recognized that gain scheduling could have been used to guarantee the stability and the desired closed loop performance for locally linearized models of the system, but this approach was not employed here.

Table 4.4 Estimation error analysis for controller neural networks

\begin{tabular}{|c|c|c|c|c|c|c|}
\hline \multicolumn{7}{|c|}{ TRAINING RESULTS } \\
\hline \multirow[t]{2}{*}{ CONTROL PARAMETER } & \multicolumn{3}{|c|}{ error } & \multicolumn{3}{|c|}{ regression } \\
\hline & e1 & e2 (응 & e3 $(\%)$ & $\mathrm{C}$ & $\mathrm{m}$ & $r 2$ \\
\hline Injection Timing (deg btdc) & 0.61 & 3.03 & -0.05 & 0.19 & 0.979 & 0.980 \\
\hline Injection Pressure (MPa) & 0.36 & 1.79 & -0.02 & 0.08 & 0.991 & 0.992 \\
\hline Fuel Flow Rate (mg/cyc) & 19.90 & 3.69 & 0.02 & 5.88 & 0.965 & 0.967 \\
\hline \multicolumn{7}{|c|}{ VALIDATION RESULTS } \\
\hline \multirow[t]{2}{*}{ CONTROL PARAMETER } & \multicolumn{3}{|c|}{ error } & \multicolumn{3}{|c|}{ Regression } \\
\hline & e1 & e2 (\%) & e3 (\%) & C & $\mathrm{m}$ & $r 2$ \\
\hline Injection Timing (deg btdc) & 0.52 & 2.59 & -1.80 & 0.13 & 1.000 & 0.986 \\
\hline Injection Pressure (MPa) & 0.37 & 1.86 & 0.93 & 0.00 & 0.991 & 0.992 \\
\hline Fuel Flow Rate (mg/cyc) & 19.70 & 3.65 & -1.55 & 9.20 & 0.942 & 0.965 \\
\hline
\end{tabular}

The pulsed torque test was simulated for six consecutive cycles at four engine speeds starting from $800 \mathrm{rpm}$ at increments of $600 \mathrm{rpm}$ and the values of the NN controller learning parameter, $\lambda$, used were $0.01,0.10$, and 1.0. The learning capability of the $\mathrm{NN}$ controller is demonstrated in 
Figure 4.11 for pulsed torque test cycle at an engine speed of $1400 \mathrm{rpm}$. A value of $\lambda$ equal to 0.10 was used for the on line adaptation. The overshoot in the actual torque value was gradually eliminated as the number of used cycles increases. The tracking performance over the fifth cycle for different engine speeds is shown in Figure 4.12. The PI controller was capable of tracking the torque pulse with very low overshoot independent of the engine speed. The expected lag in the torque rise was evident for the PI controller especially at high engine speed since the error at the previous step was used to adjust the control at the current step. On the other hand, the NN controller with sufficient learning rate did not suffer from any lag in the torque rise because of its inherent predictive ability. Of those values of $\lambda$ considered, the torque response was slightly damped for a value of 0.01 , while a value of 1.0 resulted in an oscillatory response at the tail end of the pulse, and best tracking performance was achieved for a $\lambda$ value of 0.10 , as shown in Figure 4.12. The torque tracking error and regression analysis is shown in Table 4.5 for the pulsed torque cycles. Independent of the engine speed, best tracking with the lowest values of the root mean square errors, $e_{1}$ and $e_{2}$, were achieved using the $\mathrm{NN}$ controller and $\lambda$ value of 0.1 . The $\mathrm{e}_{1}$ and $\mathrm{e}_{2}$ values for the PI controller are highest at the peak torque engine speed of $1400 \mathrm{rpm}$ and are comparable to those for the $\mathrm{NN}$ controller for a $\lambda$ value of 0.01 at other engine speeds. As expected the integral error, $\mathrm{e}_{3}$, is lowest and its mean value tracking, indicated by $\mathrm{m}$ values, is highest for the PI controller because of its integral feedback term. A comparable performance is achieved by the NN controller with a $\lambda$ value of 0.1 . The highest coefficient of determination, $r 2$, is exclusively attained using the NN controller with $\lambda=0.1$, independent of engine speed. The NN controller with a $\lambda$ value of 1.0 results in the highest bias (c) values. 
Table 4.5a Tracking error analysis over pulsed torque cycle for non-governed operation

\begin{tabular}{|c|c|c|c|c|c|c|c|c|c|c|c|c|c|}
\hline \multirow{3}{*}{$\begin{array}{c}\text { Speed } \\
\text { (rpm) }\end{array}$} & \multirow{3}{*}{ run } & \multicolumn{4}{|c|}{ e1 } & \multicolumn{4}{|c|}{ e2 (\%) } & \multicolumn{4}{|c|}{$\begin{array}{ll}\text { e3 } & \text { (\%) }\end{array}$} \\
\hline & & \multirow[t]{2}{*}{ PI } & \multicolumn{3}{|c|}{ NN } & \multirow[t]{2}{*}{ PI } & \multicolumn{3}{|c|}{ NN } & \multirow[t]{2}{*}{ PI } & \multicolumn{3}{|c|}{ NN } \\
\hline & & & 0.01 & 0.10 & 1.00 & & 0.01 & 0.10 & 1.00 & & 0.01 & 0.10 & 1.00 \\
\hline \multirow[t]{6}{*}{0800} & 1 & 25.02 & 23.48 & 15.20 & 44.24 & 3.34 & 3.13 & 2.03 & 5.90 & -2.48 & 8.48 & 2.30 & 7.15 \\
\hline & 2 & 16.63 & 15.49 & 10.74 & 21.05 & 2.22 & 2.07 & 1.43 & 2.81 & -0.10 & 6.63 & 0.93 & 2.82 \\
\hline & 3 & 15.92 & 15.83 & 10.04 & 16.67 & 2.12 & 2.11 & 1.34 & 2.22 & 0.07 & 3.78 & 0.77 & 1.10 \\
\hline & 4 & 15.87 & 16.11 & 10.48 & 15.45 & 2.12 & 2.15 & 1.40 & 2.06 & 0.02 & 3.47 & -0.16 & 0.92 \\
\hline & 5 & 15.53 & 16.02 & 10.98 & 15.80 & 2.07 & 2.14 & 1.46 & 2.11 & -0.01 & 3.36 & -0.13 & 0.80 \\
\hline & 6 & 15.84 & 16.12 & 11.20 & 16.37 & 2.11 & 2.15 & 1.49 & 2.18 & -0.05 & 3.54 & -0.02 & 0.70 \\
\hline \multirow[t]{6}{*}{1400} & 1 & 34.84 & 31.44 & 14.64 & 54.31 & 4.65 & 4.19 & 1.95 & 7.24 & -1.68 & 5.28 & 0.36 & 18.59 \\
\hline & 2 & 32.02 & 20.78 & 10.69 & 13.87 & 4.27 & 2.77 & 1.43 & 1.85 & 0.09 & -0.70 & -0.40 & 1.16 \\
\hline & 3 & 32.10 & 19.87 & 10.41 & 23.29 & 4.28 & 2.65 & 1.39 & 3.11 & -0.14 & -0.67 & -0.35 & 1.48 \\
\hline & 4 & 32.13 & 19.14 & 10.56 & 20.59 & 4.28 & 2.55 & 1.41 & 2.75 & 0.08 & -0.77 & -0.39 & 1.92 \\
\hline & 5 & 31.93 & 18.37 & 10.64 & 22.26 & 4.26 & 2.45 & 1.42 & 2.97 & -0.03 & -0.76 & -0.33 & 1.79 \\
\hline & 6 & 32.04 & 17.70 & 10.70 & 23.88 & 4.27 & 2.36 & 1.43 & 3.18 & 0.09 & -0.81 & -0.29 & 2.76 \\
\hline \multirow[t]{6}{*}{2000} & 1 & 31.15 & 43.75 & 30.42 & 119.81 & 4.15 & 5.83 & 4.06 & 15.98 & -1.41 & 6.93 & 1.52 & -3.83 \\
\hline & 2 & 28.67 & 33.68 & 20.12 & 39.76 & 3.82 & 4.49 & 2.68 & 5.30 & 0.01 & 2.91 & 2.04 & 6.18 \\
\hline & 3 & 28.92 & 31.94 & 18.81 & 33.68 & 3.86 & 4.26 & 2.51 & 4.49 & 0.00 & 3.14 & 2.57 & 3.45 \\
\hline & 4 & 28.67 & 30.25 & 17.56 & 30.12 & 3.82 & 4.03 & 2.34 & 4.02 & 0.00 & 3.08 & 2.77 & 5.70 \\
\hline & 5 & 28.27 & 28.71 & 16.70 & 27.42 & 3.77 & 3.83 & 2.23 & 3.66 & -0.01 & 3.06 & 3.14 & 5.96 \\
\hline & 6 & 28.26 & 27.36 & 16.75 & 19.69 & 3.77 & 3.65 & 2.23 & 2.63 & 0.01 & 3.03 & 3.35 & 2.14 \\
\hline \multirow[t]{6}{*}{2600} & 1 & 29.50 & 37.41 & 21.33 & 62.54 & 3.93 & 4.99 & 2.84 & 8.34 & -1.86 & 0.73 & 0.62 & 27.43 \\
\hline & 2 & 27.97 & 33.25 & 15.93 & 23.31 & 3.73 & 4.43 & 2.12 & 3.11 & 0.05 & 0.29 & -0.16 & 3.08 \\
\hline & 3 & 28.01 & 32.33 & 14.77 & 22.35 & 3.74 & 4.31 & 1.97 & 2.98 & -0.01 & 0.24 & -0.07 & 1.34 \\
\hline & 4 & 28.07 & 32.28 & 13.80 & 18.25 & 3.74 & 4.30 & 1.84 & 2.43 & -0.12 & 0.41 & -0.08 & 0.91 \\
\hline & 5 & 28.31 & 31.26 & 13.73 & 17.14 & 3.77 & 4.17 & 1.83 & 2.29 & 0.08 & 0.51 & 0.07 & 1.36 \\
\hline & 6 & 27.94 & 30.44 & 13.65 & 20.33 & 3.73 & 4.06 & 1.82 & 2.71 & -0.07 & 0.50 & 0.28 & 0.53 \\
\hline
\end{tabular}

Table 4.5b Tracking regression analysis over pulsed torque cycle for non-governed operation

\begin{tabular}{|c|c|c|c|c|c|c|c|c|c|c|c|c|c|}
\hline \multirow{3}{*}{$\begin{array}{l}\text { Speed } \\
(\text { rpm) }\end{array}$} & \multirow{3}{*}{ run } & \multicolumn{4}{|c|}{ C } & \multicolumn{4}{|c|}{$\mathrm{m}$} & \multicolumn{4}{|c|}{ r2 } \\
\hline & & \multirow[t]{2}{*}{ PI } & \multicolumn{3}{|c|}{ NN } & \multirow[t]{2}{*}{ PI } & \multicolumn{3}{|c|}{ NN } & \multirow[t]{2}{*}{ PI } & \multicolumn{3}{|c|}{ NN } \\
\hline & & & 0.01 & 0.10 & 1.00 & & 0.01 & 0.10 & 1.00 & & 0.01 & 0.10 & 1.00 \\
\hline \multirow[t]{6}{*}{0800} & 1 & 4.05 & $-11 \cdot 50$ & -3.59 & -5.88 & 0.9815 & 1.0384 & 1.0154 & 0.9915 & 0.9664 & 0.9772 & 0.9884 & 0.9042 \\
\hline & 2 & 0.79 & -3.86 & -1.12 & -3.21 & 0.9925 & 0.9751 & 1.0027 & 1.0062 & 0.9850 & 0.9892 & 0.9938 & 0.9771 \\
\hline & 3 & 0.52 & 1.78 & -0.73 & -1.02 & 0.9937 & 0.9431 & 1.0002 & 0.9999 & 0.9862 & 0.9892 & 0.9946 & 0.9851 \\
\hline & 4 & 0.59 & 2.18 & -0.22 & -0.85 & 0.9935 & 0.9419 & 1.0040 & 0.9999 & 0.9863 & 0.9886 & 0.9941 & 0.9872 \\
\hline & 5 & 0.58 & 2.20 & -0.27 & -0.68 & 0.9939 & 0.9428 & 1.0042 & 0.9993 & 0.9869 & 0.9887 & 0.9935 & 0.9866 \\
\hline & 6 & 0.71 & 1.95 & -0.24 & -0.64 & 0.9929 & 0.9437 & 1.0027 & 0.9998 & 0.9864 & 0.9885 & 0.9932 & 0.9856 \\
\hline \multirow[t]{6}{*}{1400} & 1 & 4.90 & -15.02 & -2.37 & -37.12 & 0.9806 & 1.0582 & 1.0139 & 1.0882 & 0.9688 & 0.9817 & 0.9948 & 0.9577 \\
\hline & 2 & 1.32 & 3.41 & -0.41 & -3.06 & 0.9893 & 0.9818 & 1.0070 & 1.0111 & 0.9737 & 0.9889 & 0.9971 & 0.9953 \\
\hline & 3 & 1.83 & 3.26 & -0.29 & -3.72 & 0.9879 & 0.9826 & 1.0057 & 1.0127 & 0.9735 & 0.9898 & 0.9973 & 0.9867 \\
\hline & 4 & 1.37 & 3.20 & 0.01 & -4.59 & 0.9891 & 0.9841 & 1.0038 & 1.0147 & 0.9735 & 0.9906 & 0.9972 & 0.9898 \\
\hline & 5 & 1.59 & 2.80 & 0.11 & -4.19 & 0.9886 & 0.9869 & 1.0025 & 1.0130 & 0.9738 & 0.9913 & 0.9971 & 0.9880 \\
\hline & 6 & 1.34 & 2.55 & 0.21 & -6.00 & 0.9893 & 0.9892 & 1.0014 & 1.0167 & 0.9736 & 0.9919 & 0.9971 & 0.9865 \\
\hline \multirow[t]{6}{*}{2000} & 1 & 3.75 & -6.68 & -2.02 & 8.45 & 0.9861 & 0.9805 & 0.9998 & 0.9753 & 0.9746 & 0.9525 & 0.9762 & 0.7154 \\
\hline & 2 & 0.79 & 6.02 & 1.38 & -9.86 & 0.9940 & 0.9259 & 0.9693 & 1.0118 & 0.9785 & 0.9726 & 0.9899 & 0.9625 \\
\hline & 3 & 0.93 & 5.59 & 1.81 & -5.83 & 0.9931 & 0.9269 & 0.9608 & 1.0090 & 0.9781 & 0.9759 & 0.9919 & 0.9720 \\
\hline & 4 & 0.79 & 5.36 & 1.94 & -9.37 & 0.9941 & 0.9292 & 0.9578 & 1.0129 & 0.9785 & 0.9788 & 0.9935 & 0.9787 \\
\hline & 5 & 0.83 & 5.02 & 1.47 & -6.05 & 0.9939 & 0.9320 & 0.9576 & 0.9856 & 0.9791 & 0.9811 & 0.9945 & 0.9818 \\
\hline & 6 & 0.80 & 4.69 & 1.39 & 0.81 & 0.9939 & 0.9347 & 0.9561 & 0.9725 & 0.9791 & 0.9830 & 0.9946 & 0.9903 \\
\hline \multirow[t]{6}{*}{2600} & 1 & 3.70 & -1.47 & -2.86 & -47.35 & 0.9845 & 1.0062 & 1.0202 & 1.1620 & 0.9654 & 0.9472 & 0.9831 & 0.9338 \\
\hline & 2 & 0.74 & 2.89 & 0.54 & -5.34 & 0.9927 & 0.9705 & 0.9967 & 1.0184 & 0.9690 & 0.9556 & 0.9898 & 0.9800 \\
\hline & 3 & 0.78 & 4.07 & 1.27 & -2.39 & 0.9930 & 0.9601 & 0.9890 & 1.0086 & 0.9689 & 0.9578 & 0.9912 & 0.9807 \\
\hline & 4 & 0.98 & 5.09 & 1.70 & -1.43 & 0.9921 & 0.9491 & 0.9851 & 1.0041 & 0.9688 & 0.9581 & 0.9924 & 0.9869 \\
\hline & 5 & 0.72 & 5.20 & 1.88 & -1.99 & 0.9926 & 0.9470 & 0.9820 & 1.0047 & 0.9683 & 0.9608 & 0.9925 & 0.9885 \\
\hline & 6 & 0.93 & 5.44 & 1.83 & -1.29 & 0.9921 & 0.9448 & 0.9804 & 1.0065 & 0.9690 & 0.9630 & 0.9926 & 0.9839 \\
\hline
\end{tabular}


The tracking performance over the FTP test cycle for the PI controller and the NN controller $(\lambda=0.01,0.1$, or 1.0$)$ is shown in Figure 4.13. Among the NN controllers, best tracking is achieved using $\lambda$ value of 0.1 , while a high overshoot at the sharp transients results for the case of a $\lambda$ value of 1.0, and a slightly damped tracking for $\lambda$ value of 0.01 . The NN controllers did not suffer from the same lag in the torque rise or the local instabilities demonstrated by the PI controller. The tracking error analysis of the positive torque values for the simulated as well as the measured FTP test cycle is shown in Table 4.6a. The root mean square errors, $\mathrm{e}_{1}$ and $\mathrm{e}_{2}$, are significantly lower for the NN controller $(\lambda=0.01,0.1)$ than for the PI controller. Tight tracking was predicted using the NN controller as indicated through the regression analysis. On the other hand, the PI controller, though achieved good tracking of the mean values (m value near unity), suffers from higher bias (non-zero c value) and large scatter (r2 value deviates from unity).

The FTP cycle integral results are shown in Table $4.6 \mathrm{~b}$ for the different controllers. An important result is that the tighter the tracking performance, the higher the energy expended, the higher the brake specific fuel consumption, and the higher the emissions of carbon dioxide, oxides of nitrogen, and particulate matter. The increase in the energy expended is attributed to the better tracking of the high torque values and the higher fueling rate for the NN based controller during motored operation, especially for the $\lambda$ value of 0.01 . These operating points are characterized by relatively higher brake specific $\mathrm{FC}$ and higher brake specific emissions of $\mathrm{CO}_{2}$ and $\mathrm{NOx}$, as shown in Figures 4.14, 4.15, and 4.16, respectively, at steady state operation. The increase in PM emissions is attributed to the more aggressive transients associated with the tighter tracking performance of the NN controller. Also, the better tracking of the high load operating points results in an increase in PM emissions since these points are characterized by higher brake specific PM emissions, as shown in Figure 4.17, which are averaged over transient operation. The results for 
THC and LCO, shown in Table 4.6b, must be treated with caution in view of their less satisfactory prediction accuracy demonstrated in the previous chapter. The increase in THC emissions for the $\mathrm{NN}$ controller with $\lambda$ value of 0.01 is attributed to the higher fueling at motored operation where the rate of change in brake specific THC emissions is highest, as shown in Figure 4.18, at steady state operation. The decrease in LCO emissions for the $\mathrm{NN}$ controller is attributed to the better tracking of the high-load low-speed operation where brake specific emissions of LCO decline as shown in Figure 4.19 at steady state operation.

Table 4.6a Tracking analysis for positive torque over the FTP for non-governed operation

\begin{tabular}{|c|c|c|c|c|c|c|}
\hline \multirow{3}{*}{ Measure } & \multicolumn{4}{|c|}{ SIMULATION } & \multicolumn{2}{|c|}{ MEASUREMENT } \\
\hline & \multirow[t]{2}{*}{ PI } & \multicolumn{3}{|c|}{ NN } & \multirow[t]{2}{*}{ RUN1 } & \multirow[t]{2}{*}{ RUN2 } \\
\hline & & 0.01 & 0.10 & 1.00 & & \\
\hline$e 1 \quad(f t-l b f)$ & 50.17 & 16.35 & 11.86 & 40.08 & 46.95 & 35.64 \\
\hline $\begin{array}{ll}e 2 & (\circ) \\
\end{array}$ & 6.69 & 2.18 & 1.58 & 5.34 & 6.26 & 4.75 \\
\hline $\begin{array}{ll}\text { e3 } & (\%) \\
\end{array}$ & 9.97 & 2.87 & 1.45 & 6.34 & 8.69 & 6.79 \\
\hline$c \quad(\mathrm{ft}-1 \mathrm{bf})$ & -10.99 & -4.33 & -2.50 & -7.80 & -5.20 & -4.79 \\
\hline$m(n / a)$ & 0.99732 & 1.00954 & 1.00759 & 1.00542 & 0.95898 & 0.97433 \\
\hline r2 (n/a) & 0.88513 & 0.98665 & 0.99276 & 0.92328 & 0.89125 & 0.93612 \\
\hline
\end{tabular}

Table 4.6b Integral results over the FTP cycle for non-governed operation

\begin{tabular}{|c|c|c|c|c|c|c|}
\hline \multirow{3}{*}{ PARMETER } & \multicolumn{4}{|c|}{ SIMULATION } & \multicolumn{2}{|c|}{ MEASUREMENT } \\
\hline & \multirow[t]{2}{*}{ PI } & \multicolumn{3}{|c|}{ NN } & \multirow[t]{2}{*}{ RUN1 } & \multirow[t]{2}{*}{ RUN2 } \\
\hline & & 0.01 & 0.10 & 1.00 & & \\
\hline ENERGY (bhp-h) & 9.097 & 9.990 & 9.952 & 9.394 & 10.096 & 9.991 \\
\hline ENERGY+ (bhp-h) & 11.023 & 11.299 & 11.348 & 11.157 & 11.195 & 11.159 \\
\hline FC $(g / b h p-h)$ & 178.912 & 190.584 & 187.670 & 183.160 & 194.787 & 189.999 \\
\hline THC $(g / b h p-h)$ & 0.208 & 0.215 & 0.197 & 0.186 & 0.214 & 0.203 \\
\hline $\mathrm{LCO}(\mathrm{g} / \mathrm{bhp}-\mathrm{h})$ & 2.525 & 1.834 & 1.940 & 1.857 & 1.889 & 1.573 \\
\hline $\mathrm{CO}_{2}(\mathrm{~g} / \mathrm{bhp}-\mathrm{h})$ & 604.356 & 661.082 & 643.313 & 634.714 & 674.755 & 650.069 \\
\hline NOx $(g / b h p-h)$ & 5.084 & 5.607 & 5.469 & 5.254 & 5.494 & 5.786 \\
\hline PM $(g / b h p-h)$ & 0.081 & 0.079 & 0.092 & 0.090 & 0.127 & 0.080 \\
\hline
\end{tabular}

The positive torque tracking performance error analysis over the FTP and the arbitrary test cycles A through $\mathrm{H}$ is shown in Table $4.7 \mathrm{a}$ for the PI controller and the $\mathrm{NN}$ controller $(\lambda=0.1)$. As before, the NN controller is capable of achieving much tighter control independent of the test cycle with much lower bias and scatter compared to that achieved using the PI controller. 
Table 4.7a Positive torque tracking error analysis over transient non-governed operation

\begin{tabular}{|c|c|c|c|c|c|c|c|c|c|c|c|c|}
\hline \multirow{3}{*}{ cycle } & \multicolumn{6}{|c|}{ Error } & \multicolumn{6}{|c|}{ Regression } \\
\hline & \multicolumn{2}{|c|}{ e1 } & \multicolumn{2}{|c|}{ e2 (\%) } & \multicolumn{2}{|c|}{ e3 (\%) } & \multicolumn{2}{|l|}{$\mathrm{C}$} & \multicolumn{2}{|c|}{$\mathrm{m}$} & \multicolumn{2}{|c|}{$r 2$} \\
\hline & PI & $\mathrm{NN}$ & \begin{tabular}{l|l}
$\mathrm{PI}$ & \\
\end{tabular} & NN & $\mathrm{PI}$ & NN & PI & NN & PI & $\mathrm{NN}$ & $\mathrm{PI}$ & NN \\
\hline FTP & 50.17 & 11.86 & 6.69 & 1.58 & 9.97 & 1.45 & -10.99 & -2.50 & 0.9973 & 1.0076 & 0.8851 & 0.9928 \\
\hline ARB A & 40.53 & 13.83 & 5.40 & 1.84 & 1.35 & 1.13 & -5.09 & -4.99 & 1.0164 & 1.0181 & 0.9279 & 0.9915 \\
\hline$A R B \quad B$ & 31.89 & 7.41 & 4.25 & 0.99 & -0.04 & 0.20 & 0.21 & -0.78 & 0.9992 & 1.0025 & 0.9511 & 0.9973 \\
\hline $\mathrm{ARB} \quad \mathrm{C}$ & 26.68 & 12.03 & 3.56 & 1.60 & -0.07 & 0.26 & 0.62 & -1.79 & 0.9974 & 1.0070 & 0.9619 & 0.9922 \\
\hline ARB D & 32.68 & 15.80 & 4.36 & 2.11 & -0.08 & -0.08 & 0.95 & -0.65 & 0.9950 & 1.0048 & 0.9411 & 0.9859 \\
\hline ARB E & 24.29 & 11.12 & 3.24 & 1.48 & -0.04 & 0.08 & 0.20 & -0.39 & 0.9993 & 1.0013 & 0.9668 & 0.9929 \\
\hline $\begin{array}{ll}A R B \quad F \\
\end{array}$ & 27.74 & 13.03 & 3.70 & 1.74 & -0.03 & -0.02 & 0.39 & 0.05 & 0.9982 & 1.0000 & 0.9597 & 0.9909 \\
\hline ARB G & 27.39 & 15.00 & 3.65 & 2.00 & -0.03 & -0.07 & 0.50 & 0.02 & 0.9975 & 1.0005 & 0.9606 & 0.9879 \\
\hline ARB H & 26.98 & 14.67 & 3.60 & 1.96 & -0.08 & 0.08 & 0.71 & -0.76 & 0.9971 & 1.0032 & 0.9617 & 0.9885 \\
\hline
\end{tabular}

Table 4.7b Performance integral results over transient non-governed operation

\begin{tabular}{|c|c|c|c|c|c|c|c|c|c|}
\hline \multirow{3}{*}{ cycle } & \multicolumn{3}{|c|}{ energy (bhp-h) } & \multicolumn{3}{|c|}{ pos. energy (bhp-h) } & \multicolumn{3}{|c|}{ EC $(g / b h p-h)$} \\
\hline & \multicolumn{2}{|c|}{ SIM } & \multirow[t]{2}{*}{ MES } & \multicolumn{2}{|c|}{ SIM } & \multirow[t]{2}{*}{ MES } & \multicolumn{2}{|c|}{ SIM } & \multirow[t]{2}{*}{ MES } \\
\hline & $\mathrm{PI}$ & NN & & $\mathrm{PI}$ & NN & & $\mathrm{PI}$ & NN & \\
\hline FTP & 9.10 & 9.95 & 10.05 & 11.02 & 11.35 & 11.26 & 178.91 & 187.67 & 191.08 \\
\hline $\mathrm{ARB} \quad \mathrm{A}$ & 7.99 & 8.13 & 8.24 & 10.55 & 10.52 & 10.63 & 187.96 & 198.46 & 196.13 \\
\hline$A R B \quad B$ & 13.39 & 13.38 & 13.46 & 14.27 & 14.18 & 14.39 & 188.94 & 190.27 & 187.32 \\
\hline $\mathrm{ARB} C$ & 8.36 & 8.36 & 8.30 & 8.79 & 8.78 & 8.76 & 181.21 & 181.84 & 179.65 \\
\hline $\mathrm{ARB} \quad \mathrm{D}$ & 5.31 & 5.30 & 5.09 & 5.34 & 5.32 & 5.21 & 172.28 & 171.57 & 169.34 \\
\hline ARB E & 10.63 & 10.64 & 10.48 & 10.67 & 10.66 & 10.56 & 167.08 & 166.03 & 165.03 \\
\hline$\overline{A R B} \quad \mathrm{~F}$ & 14.13 & 14.14 & 13.86 & 14.21 & 14.19 & 14.06 & 171.49 & 170.80 & 168.66 \\
\hline ARB G & 13.79 & 13.81 & 13.37 & 13.86 & 13.85 & 13.37 & 173.23 & 172.26 & 170.65 \\
\hline ARB H & 15.27 & 15.30 & 14.74 & 15.92 & 15.91 & 15.86 & 180.41 & 180.38 & 172.96 \\
\hline
\end{tabular}

Table 4.7c Emissions integral results (g/bhp-h) over transient non-governed operation

\begin{tabular}{|c|c|c|c|c|c|c|c|c|c|c|c|c|c|c|c|}
\hline \multirow{3}{*}{ Cycle } & \multicolumn{3}{|c|}{ THC } & \multicolumn{3}{|c|}{ LCO } & \multicolumn{3}{|c|}{$\mathrm{CO}_{2}$} & \multicolumn{3}{|c|}{ NOx } & \multicolumn{3}{|c|}{ PM } \\
\hline & \multicolumn{2}{|c|}{ SIM } & \multirow[t]{2}{*}{ MES } & \multicolumn{2}{|c|}{ SIM } & \multirow[t]{2}{*}{ MES } & \multicolumn{2}{|c|}{ SIM } & \multirow[t]{2}{*}{ MES } & \multicolumn{2}{|c|}{ SIM } & \multirow[t]{2}{*}{ MES } & \multicolumn{2}{|c|}{ SIM } & \multirow[t]{2}{*}{ MES } \\
\hline & PI & NN & & PI & NN & & PI & NN & & PI & NN & & PI & NN & \\
\hline FTP & 0.21 & 0.20 & 0.21 & 2.52 & 1.94 & 1.72 & 604.36 & 643.31 & 657.14 & 5.08 & 5.47 & 5.60 & 0.081 & 0.092 & 0.102 \\
\hline$\overline{A R B ~ A}$ & 0.24 & 0.26 & 0.26 & 2.83 & 2.81 & 3.25 & 626.70 & 659.50 & 649.46 & 5.09 & 5.59 & 5.52 & .095 & .120 & \\
\hline$\overline{A R B \quad B}$ & 0.23 & 0.22 & 0.28 & 2.32 & 2.37 & 2.42 & 638.36 & 661.15 & 652.53 & 5.43 & 5.54 & 5.50 & 0.103 & 0.126 & \\
\hline ARB C & 0.20 & 0.19 & 0.18 & 2.32 & 2.40 & 3.00 & 627.74 & 639.18 & 642.02 & 5.51 & 5.57 & 5.46 & 0.091 & 0.101 & 0.097 \\
\hline ARB D & 0.14 & 0.13 & 0.13 & 2.13 & 2.29 & 1.66 & 624.36 & 634.65 & 643.11 & 5.51 & 5.54 & 5.39 & 0.090 & 0.099 & 0.086 \\
\hline$\overline{A R B} E$ & 0.13 & 0.12 & 0.12 & 1.70 & 1.78 & 1.55 & 609.00 & 620.92 & 624.15 & 5.46 & 5.48 & 5.34 & 0.082 & 0.092 & 0.083 \\
\hline$\overline{A R B \quad F}$ & 0.14 & 0.13 & 0.13 & 1.78 & 1.88 & 1.71 & 619.96 & 633.13 & 630.73 & 5.43 & 5.47 & 5.33 & 0.086 & 0.098 & 0.091 \\
\hline$\overline{A R B \quad G}$ & 0.14 & 0.13 & 0.13 & 1.74 & 1.89 & 1.62 & 625.97 & 639.58 & 634.13 & 5.48 & 5.50 & 5.68 & 0.079 & 0.091 & 0.083 \\
\hline$\overline{A R B ~ H}$ & 0.18 & 0.17 & 0.16 & 1.98 & 2.18 & 1.78 & 629.03 & 643.40 & 621.48 & 5.41 & 5.46 & 5.55 & 0.093 & 0.109 & 0.094 \\
\hline
\end{tabular}

The integral results over the transient cycles for engine operation and emissions are shown in Table $4.7 \mathrm{~b}$ and Table $4.7 \mathrm{c}$, respectively. The brake specific fuel consumption is higher for the FTP and the test cycles A through $\mathrm{C}$ due to the higher fueling for the $\mathrm{NN}$ controller during the motored operation whereas the cycles D through G did not include any motoring operation, as was shown in Table 2.1. The tight tracking of the torque schedule results in increased emissions of $\mathrm{CO}_{2}, \mathrm{NOx}$, and PM independent of the test cycle due to the more aggressive transients associated with $\mathrm{NN}$ based controller. 
Several simulations were conducted for biased desired torque values over the FTP test cycle using the NN controller $(\lambda=0.1)$. The purpose of these simulations was to investigate the variations in the integral results over the FTP cycle for the EPA allowed range in the test validation criteria, shown in Table 4.2. This investigation was motivated by the ability of the NN-based controller to achieve any reasonably biased desired torque schedule while still meeting the EPA validation criteria. The prescribed values were either shifted within the validation criteria intercept range $(\mathrm{c}=$ \pm 15 ), deviated within a regression slope in the range 0.80 to 1.05 (validation range $\mathrm{m}=0.83$ to $1.03)$, or both.

Table 4.8 Integral results over the FTP cycle for biased desired schedule

\begin{tabular}{|c|c|c|c|c|c|c|c|c|c|}
\hline \multicolumn{2}{|c|}{ Regression } & \multicolumn{2}{|c|}{ energy $(\mathrm{bhp}-\mathrm{h})$} & \multirow{2}{*}{$\frac{F C}{(g / b h p-h)}$} & \multicolumn{5}{|c|}{ emissions $(\mathrm{g} / \mathrm{bhp}-\mathrm{h})$} \\
\hline Intercept & slope & total & positive & & THC & \begin{tabular}{|l|l|}
$\mathrm{LCO}$ \\
\end{tabular} & $\mathrm{CO}_{2}$ & NOx & PM \\
\hline-17.8 & 1.058 & 9.42 & 11.12 & 186.68 & 0.20 & 1.62 & 644.67 & 5.42 & 0.076 \\
\hline $\mid-17.8$ & 1.008 & 8.86 & 10.55 & 188.86 & 0.21 & 1.59 & 648.89 & 5.51 & 0.071 \\
\hline-17.7 & 0.907 & 7.73 & 9.41 & 195.84 & 0.25 & 1.60 & 664.97 & 5.74 & 0.068 \\
\hline-17.5 & 0.806 & 6.62 & 8.28 & 205.87 & 0.29 & 1.73 & 687.89 & 6.00 & 0.071 \\
\hline-7.7 & 1.057 & 10.14 & 11.63 & 186.33 & 0.19 & 1.75 & 643.09 & 5.42 & 0.085 \\
\hline $\mid-7.6$ & 1.008 & 9.59 & 11.07 & 187.98 & 0.20 & 1.71 & 645.46 & 5.50 & 0.079 \\
\hline-7.4 & 0.907 & 8.47 & 9.94 & 194.14 & 0.23 & 1.72 & 659.29 & 5.72 & 0.075 \\
\hline-7.2 & 0.806 & 7.36 & 8.80 & 203.11 & 0.27 & 1.81 & 680.39 & 5.98 & 0.077 \\
\hline-2.5 & 1.056 & 10.50 & 11.91 & 186.22 & 0.19 & 1.98 & 642.26 & 5.40 & 0.097 \\
\hline-2.5 & 1.008 & 9.95 & 11.35 & 187.67 & 0.20 & 1.94 & 643.31 & 5.47 & 0.092 \\
\hline-2.3 & 0.907 & 8.84 & 10.21 & 193.37 & 0.23 & 1.96 & 654.98 & 5.67 & 0.088 \\
\hline-2.1 & 0.806 & 7.73 & 9.07 & 202.16 & 0.26 & 2.10 & 673.34 & 5.92 & 0.092 \\
\hline 2.9 & 1.054 & 10.86 & 12.23 & 182.64 & 0.18 & 1.76 & 679.10 & 5.48 & 0.088 \\
\hline 2.8 & 1.006 & 10.32 & 11.68 & 183.76 & 0.20 & 1.71 & 681.71 & 5.55 & 0.082 \\
\hline 3.0 & 0.906 & 9.21 & 10.55 & 188.58 & 0.22 & 1.68 & 695.53 & 5.76 & 0.076 \\
\hline 3.2 & 0.805 & 8.11 & 9.41 & 196.29 & 0.26 & 1.76 & 718.59 & 6.01 & 0.077 \\
\hline 13.4 & 1.051 & 11.59 & 12.91 & 180.13 & 0.17 & 1.84 & 675.88 & 5.44 & 0.091 \\
\hline 13.2 & 1.005 & 11.06 & 12.37 & 180.72 & 0.18 & 1.77 & 677.09 & 5.50 & 0.084 \\
\hline 13.3 & 0.905 & 9.96 & 11.24 & 184.21 & 0.21 & 1.72 & 688.27 & 5.69 & 0.075 \\
\hline 13.5 & 0.804 & 8.86 & 10.10 & 190.71 & 0.24 & 1.79 & 709.13 & 5.93 & 0.074 \\
\hline
\end{tabular}

The integral results over the FTP cycle are shown in Table 4.8 and the percent deviations from the case of the unbiased schedule are shown in Figure 4.20, where the shaded area indicates deviations allowed within the EPA test validation criteria. An expected result was the increase in the expended energy (both total and positive) with an increase in $\mathrm{c}$ and $\mathrm{m}$ values, as shown in Figure $4.20 \mathrm{a}$ and $4.20 \mathrm{~b}$, and the allowable range of variation was between $-20 \%$ and $10 \%$ for the EPA validation criteria given in Table 4.2. The allowable deviation in FC was between $-3 \%$ and 
5\% with higher brake specific fuel consumption at lower levels of expended energy, as shown in Figure 4.20c. The range of variation in THC emissions within the EPA validation criteria was between $-3 \%$ to $25 \%$ with higher emissions for the lower intercept and slope values, as shown in Figure 4.20d. The variation in $\mathrm{CO}$ emissions was between $-15 \%$ and $4 \%$ with the highest emissions for the unbiased schedule, as shown in Figure 4.20e. This result agreed with the steady state map, shown in Figure 4.19a, where CO emissions had a distinctive peak at the low speed and high load operation. The deviation in $\mathrm{CO}_{2}$ and NOx emissions is shown in Figures 4.20f and 4.20g, respectively. The deviations within the EPA validation criteria were up to $8 \%$ for $\mathrm{CO}_{2}$ and $-1 \%$ to $6 \%$ for NOx with a distinctive minimum for the unbiased schedule $(\mathrm{c}=0)$ for a given $\mathrm{m}$ value while emissions were higher for a lower slope of the regression. The opposite trend is shown in Figure 4.20h for the PM emissions with a distinctive peak for $\mathrm{c}=0$ and a given $\mathrm{m}$ value, and higher PM emissions for lower $\mathrm{m}$ values. The variation in PM emissions was between $-25 \%$ and $0 \%$ for the EPA validation criteria.

In summary, conducting a test that meets the EPA validation criteria for the FTP still allows for a reasonable variation in the brake specific emissions ranging from 5\% for $\mathrm{CO}_{2}$ and $\mathrm{NOx}$ emissions, up to $15 \%$ for $\mathrm{CO}$ emissions and $25 \%$ for PM and THC emissions. The allowable deviation in the integral energy is quite high, reaching up to $25 \%$ for both total and positive expended energy. It must be stressed that the specific reported percentile deviations are strongly dependent on the engine calibration and would vary for other test engines. Also, since the reported results are not validated through actual experiments, caution must be exercised especially for predictions of THC and $\mathrm{CO}$ emissions. 


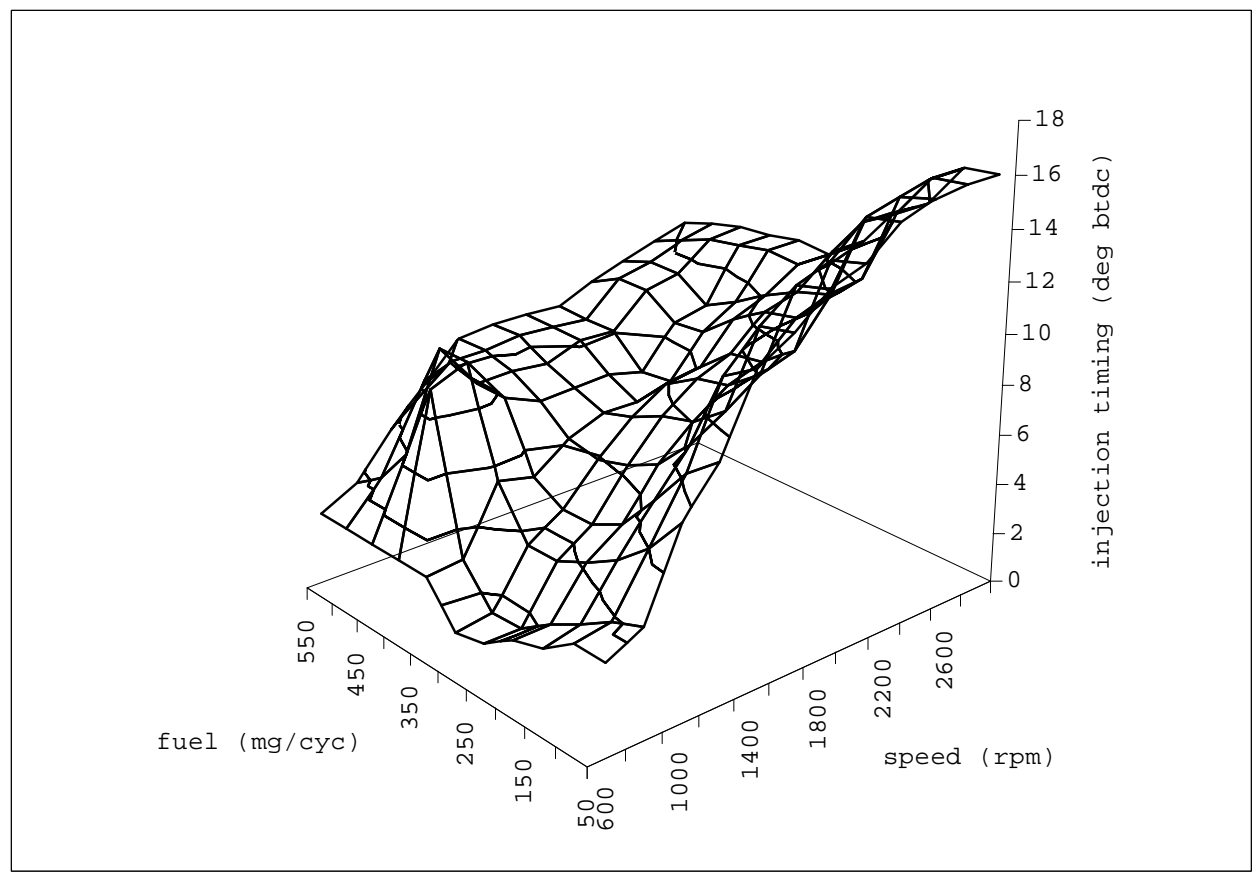

Figure 4.5a Injection timing map

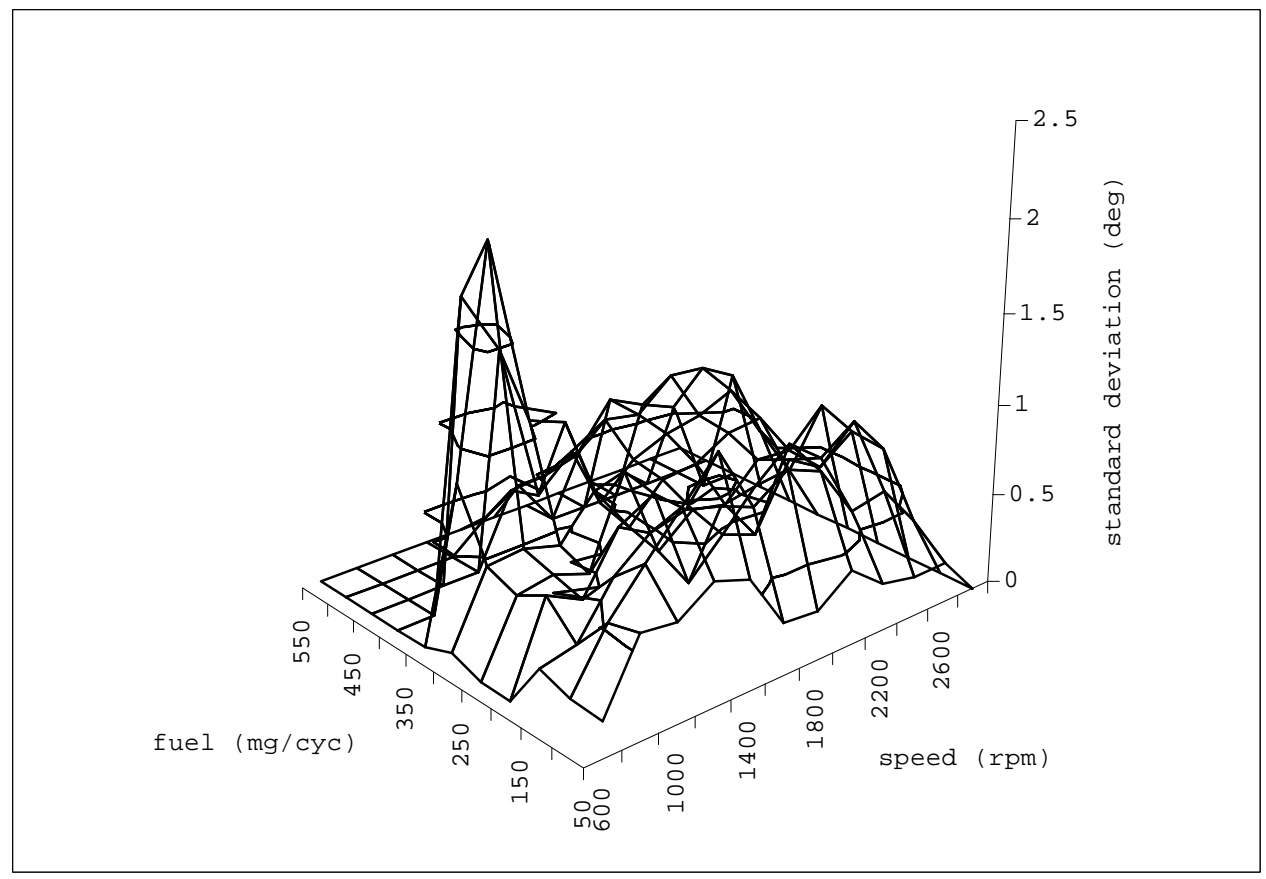

Figure 4.5b Standard deviation distribution of the injection timing map 


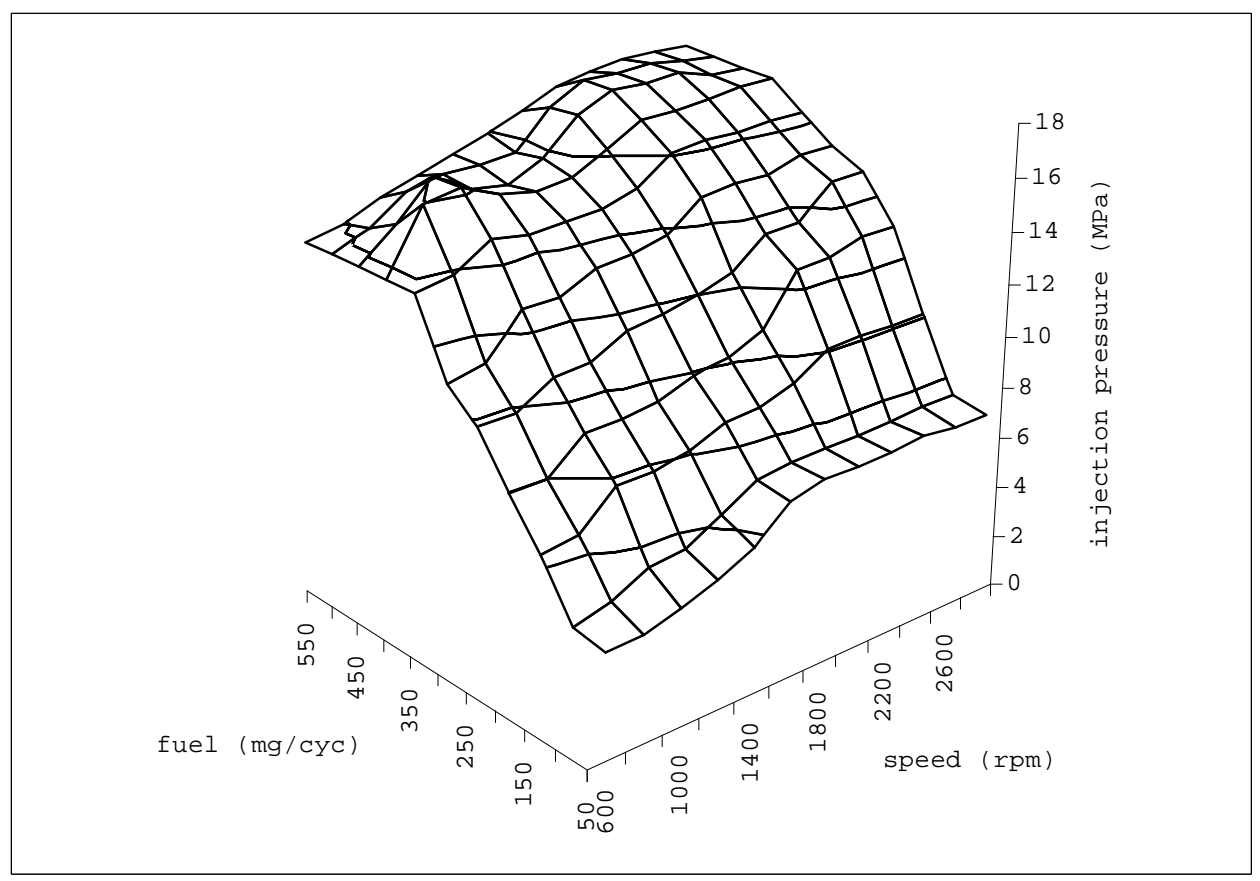

Figure 4.6a Injection pressure map

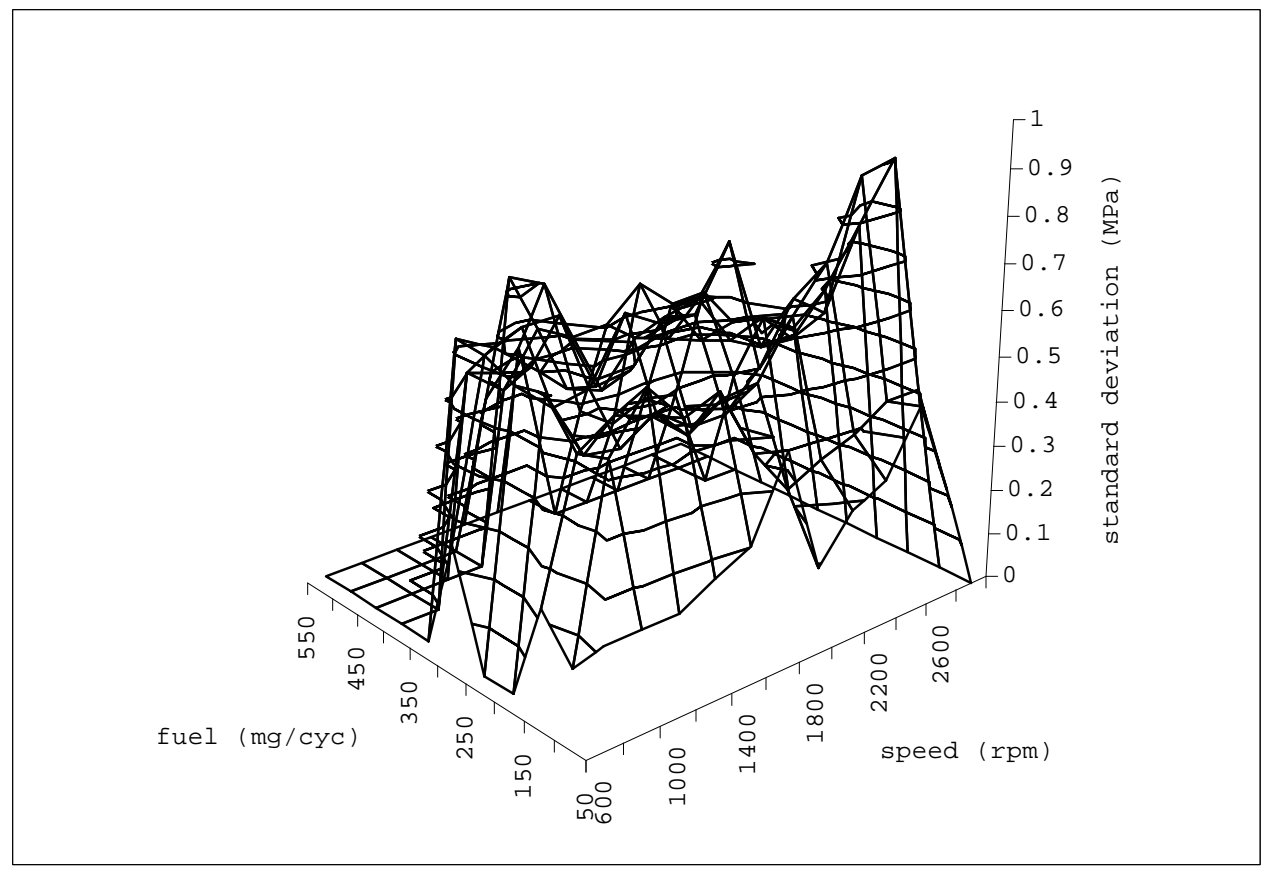

Figure 4.6b Standard deviation distribution of the injection pressure map 


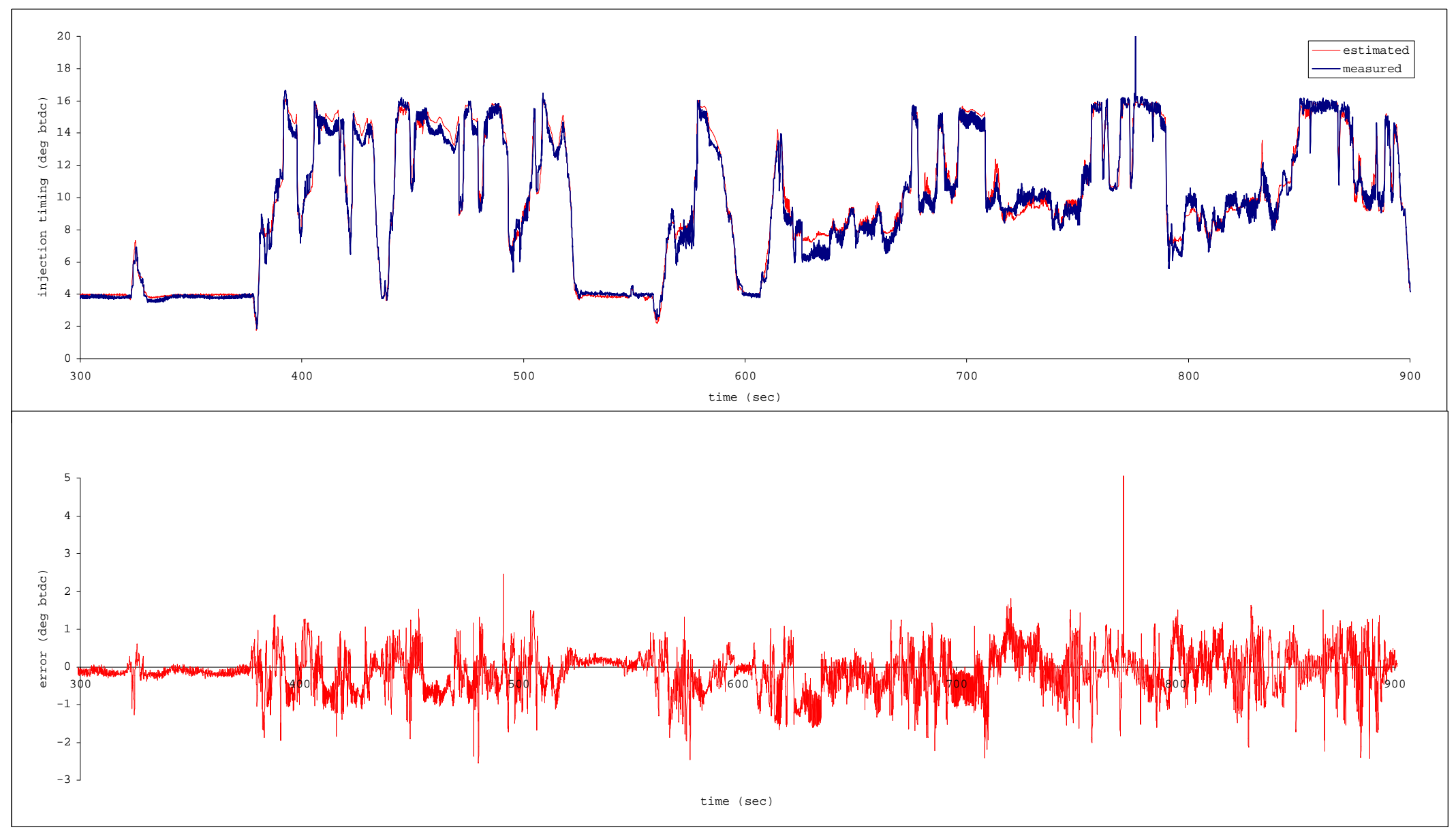

Figure 4.7 Measured and estimated injection timing over the FTP 

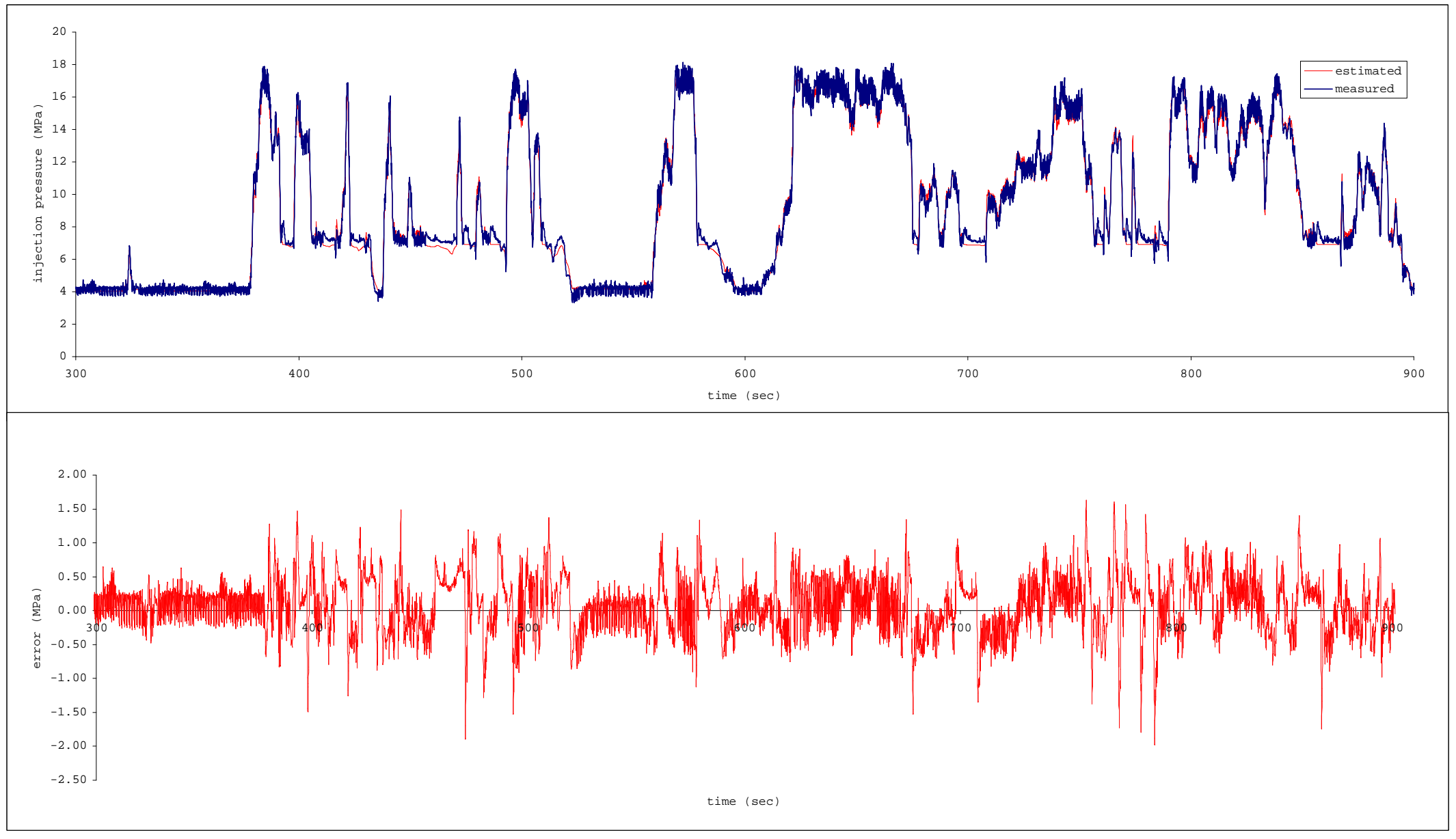

Figure 4.8 Measured and estimated injection pressure over the FTP cycle 


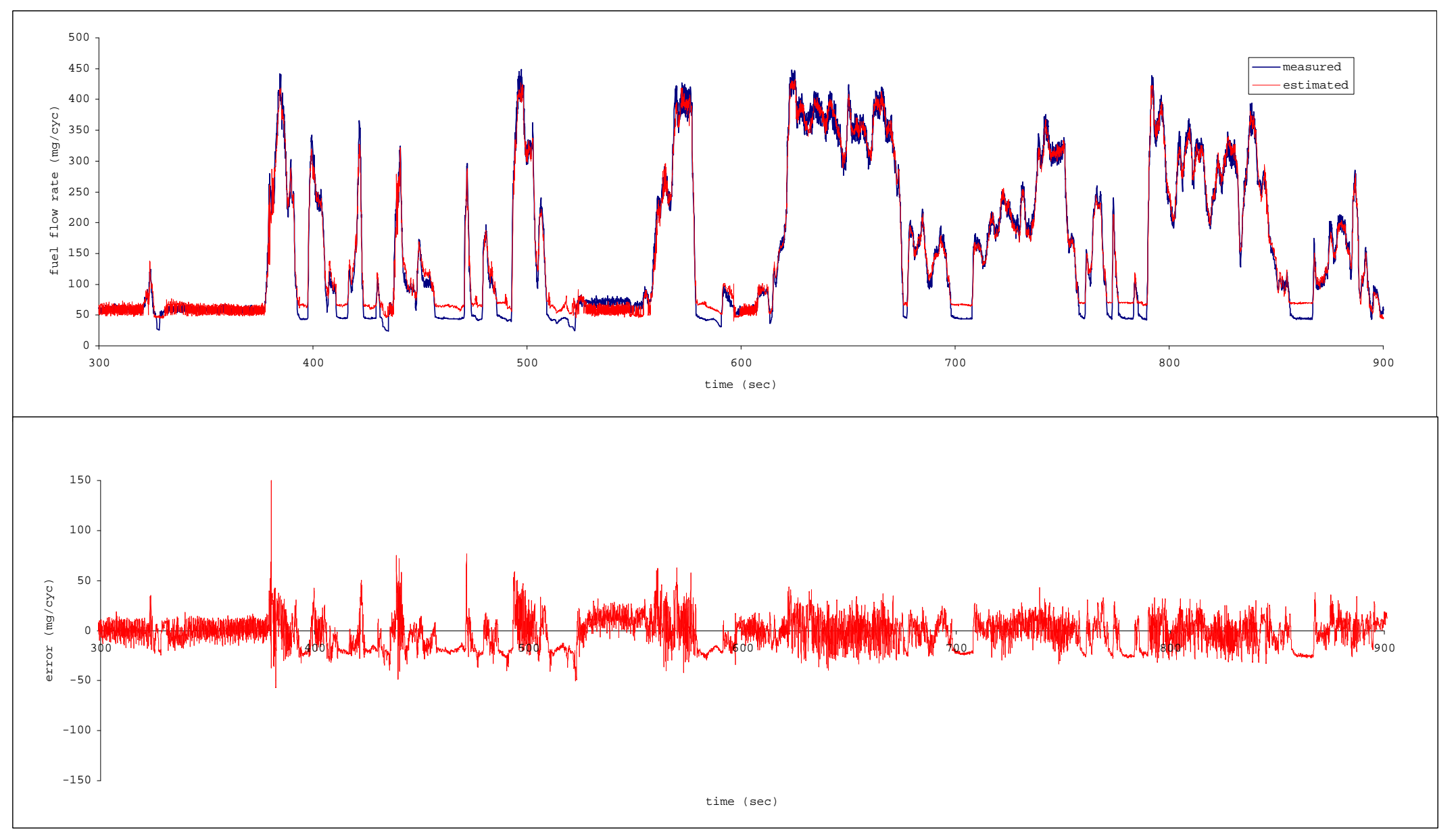

Figure 4.9 Measured and estimated fuel mass flow rate over the FTP cycle

105 


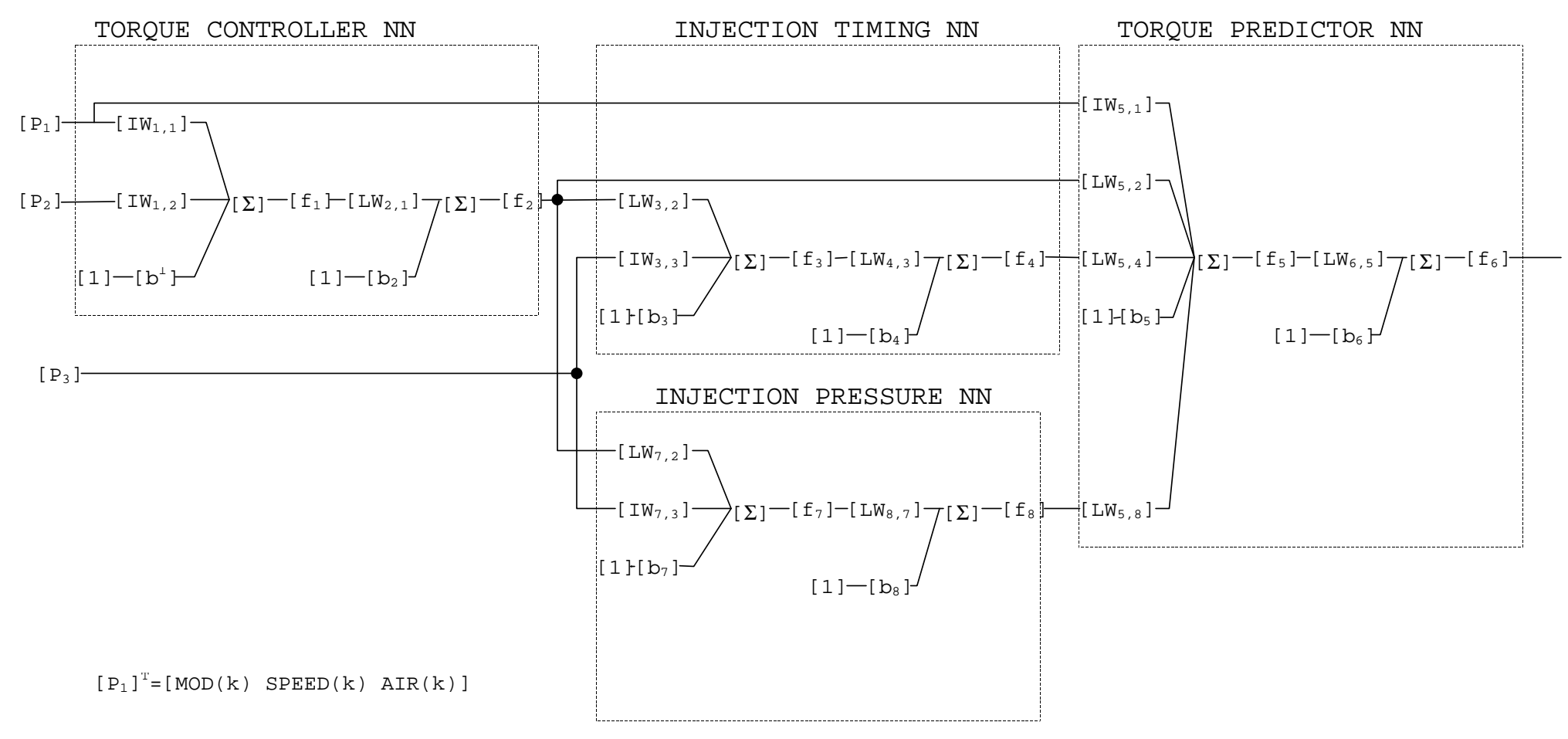

$\left[P_{2}\right]^{T}=[\operatorname{SPEED}(k+1) \quad$ TORQref $(k+1)]$

$\left[\mathrm{P}_{3}\right]=[\operatorname{SPEED}(k)]$

Figure 4.10 Combined neural network layout for non-governed engine operation 


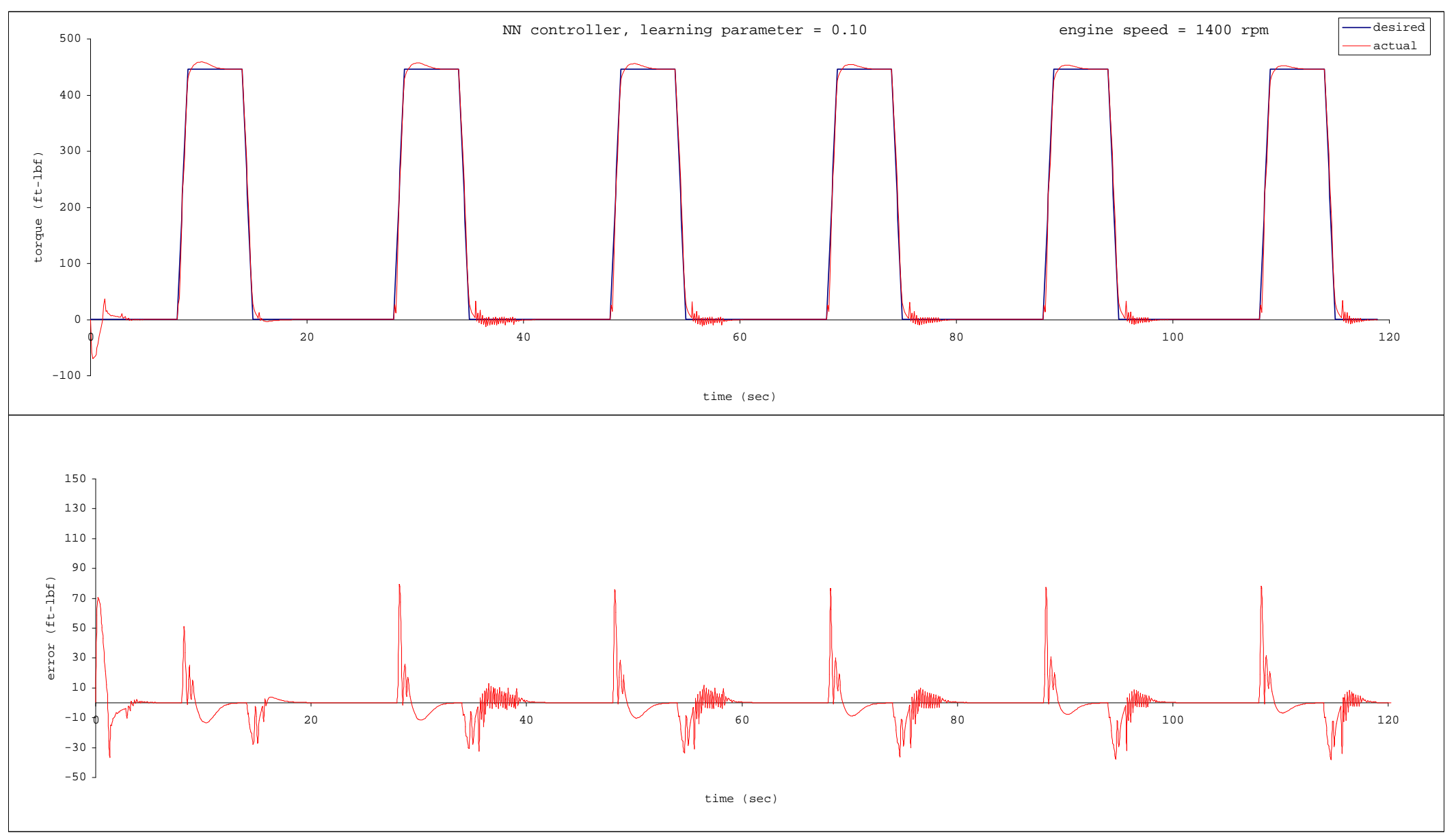

Figure 4.11 Torque tracking performance over the repeated pulsed torque test cycle for non-governed operation 

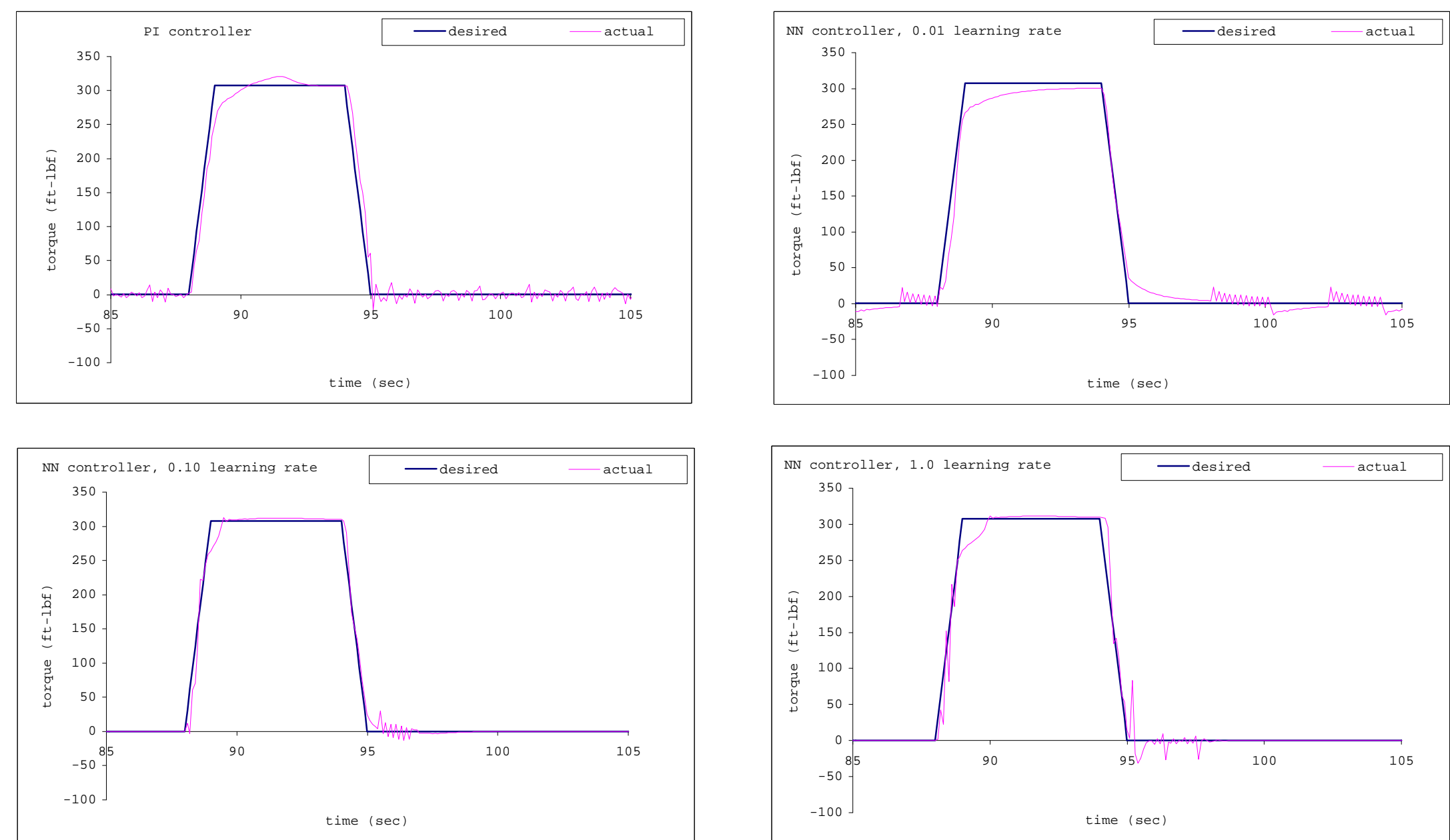

Figure 4.12a Torque tracking performance over the repaeted pulsed torque test cycle at $800 \mathrm{rpm}$ for non-governed operation 

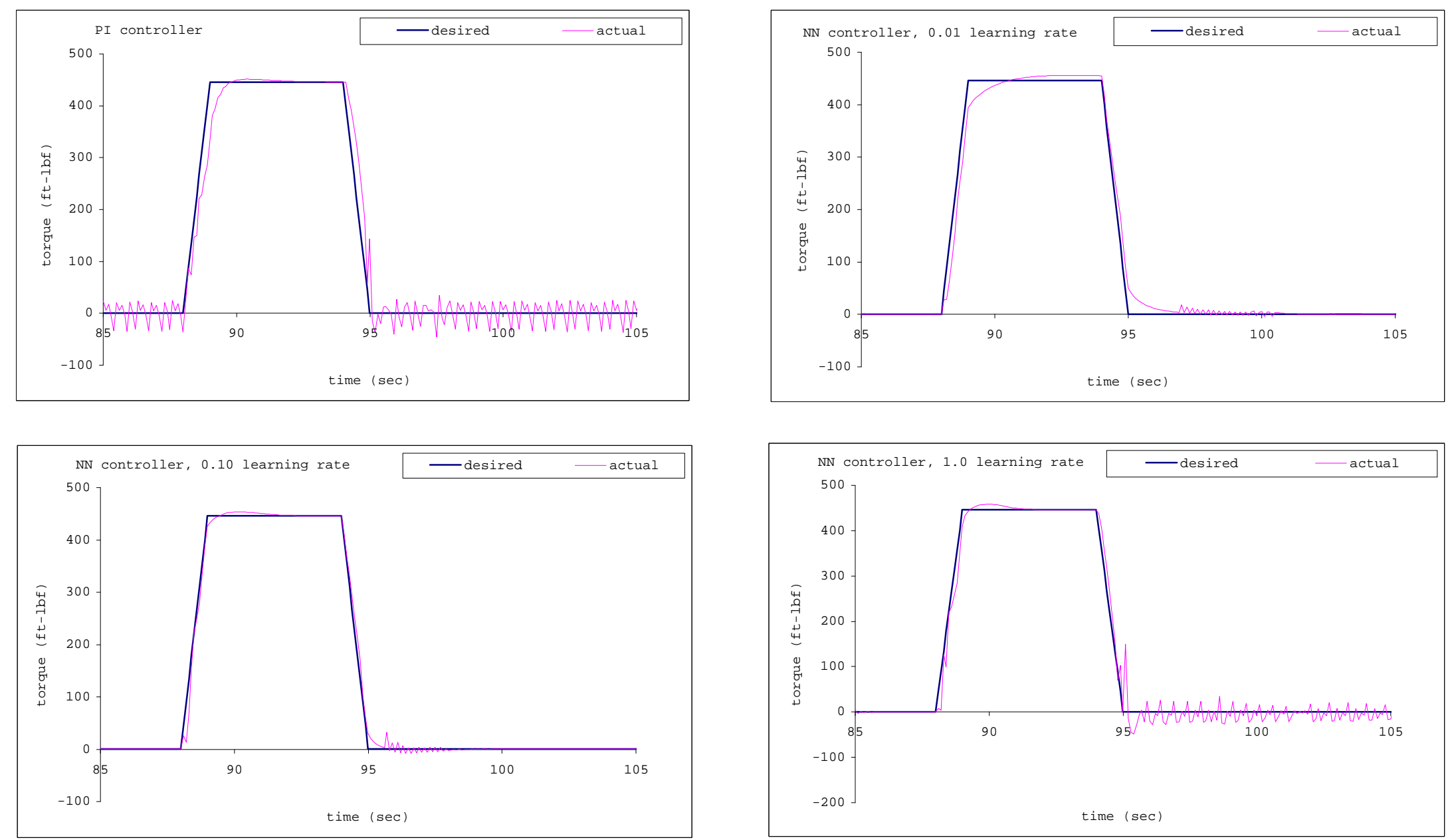

Figure 4.12b Torque tracking performance over the repeated pulsed torque test cycle at $1400 \mathrm{rpm}$ for non-governed operation 

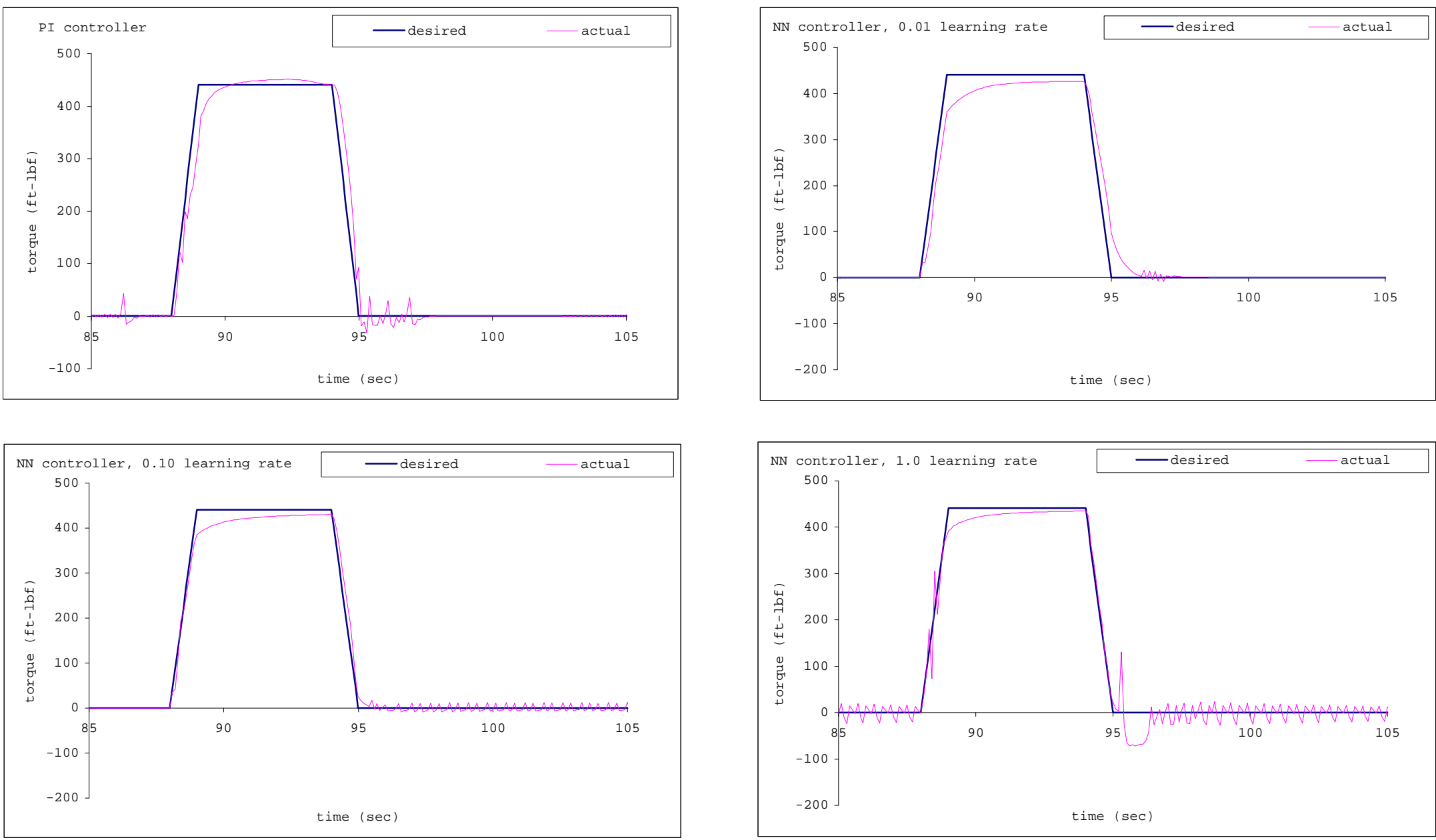

Figure 4.12c Torque tracking performance over the repeated pulsed torque test cycle at $2000 \mathrm{rpm}$ for non-governed operation 

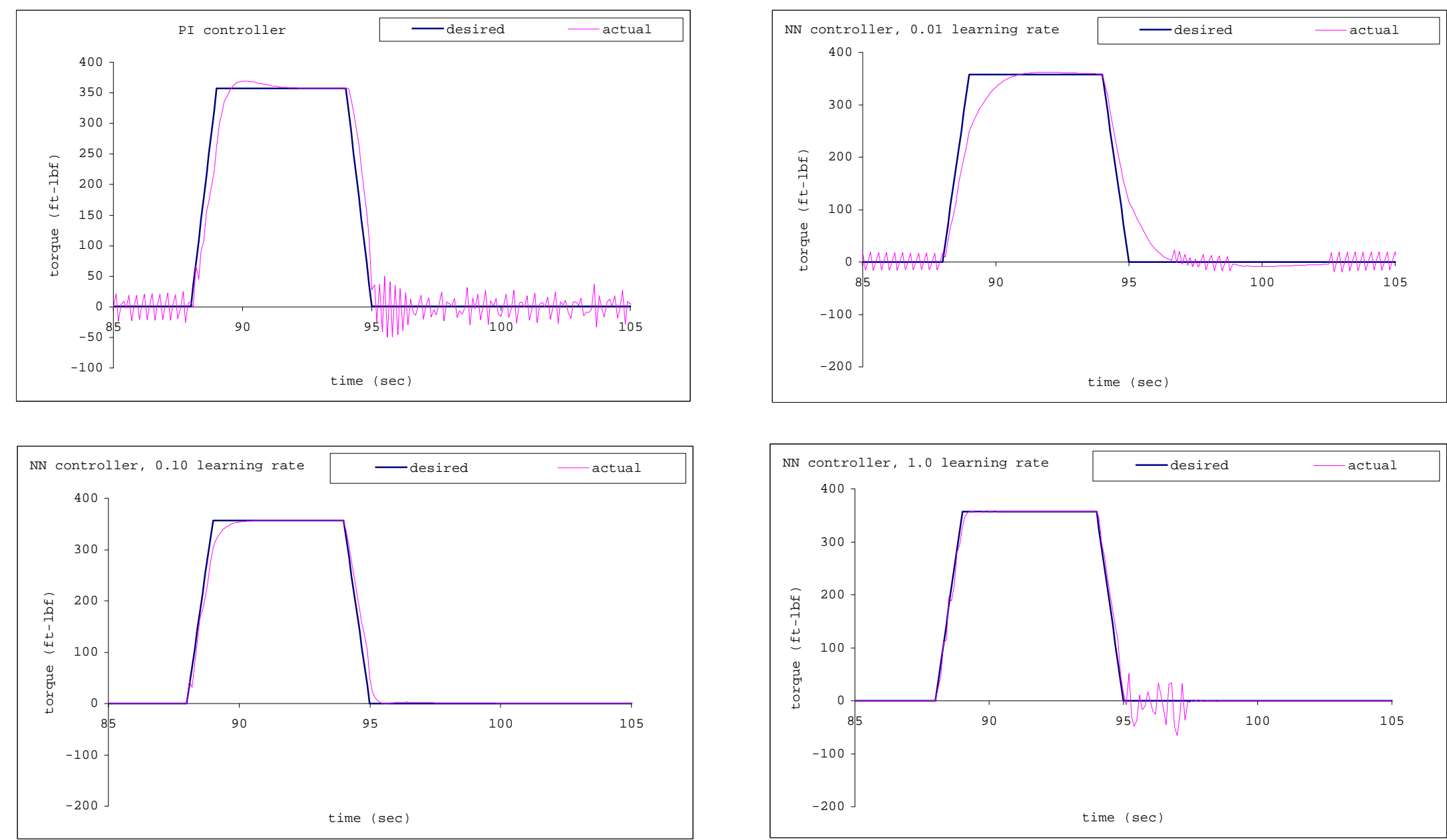

Figure 4.12d Torque tracking performance over the repeated pulsed torque test cycle at $2600 \mathrm{rpm}$ for non-governed operation 


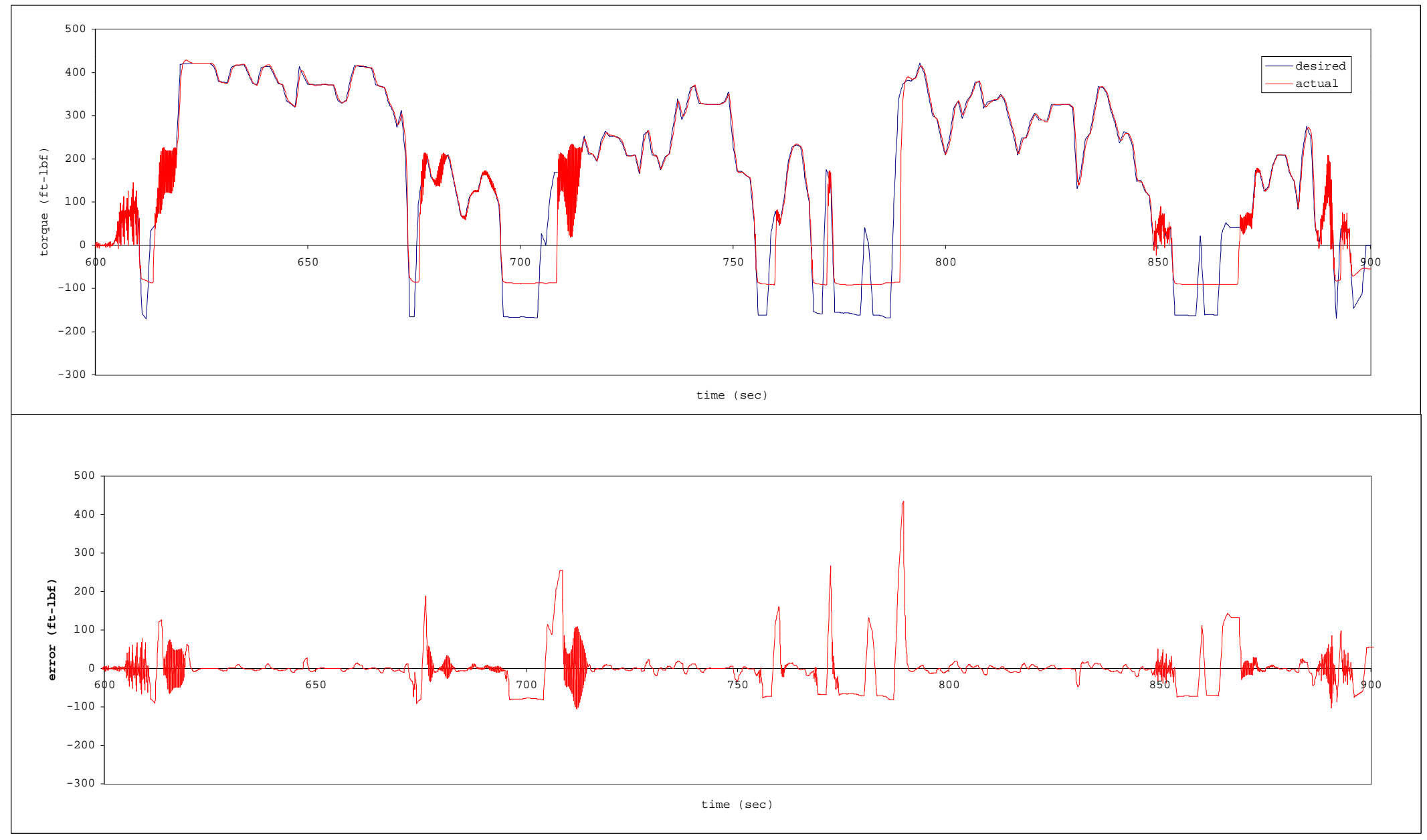

Figure 4.11a Desired and actual torque over the FTP cycle for PI control 


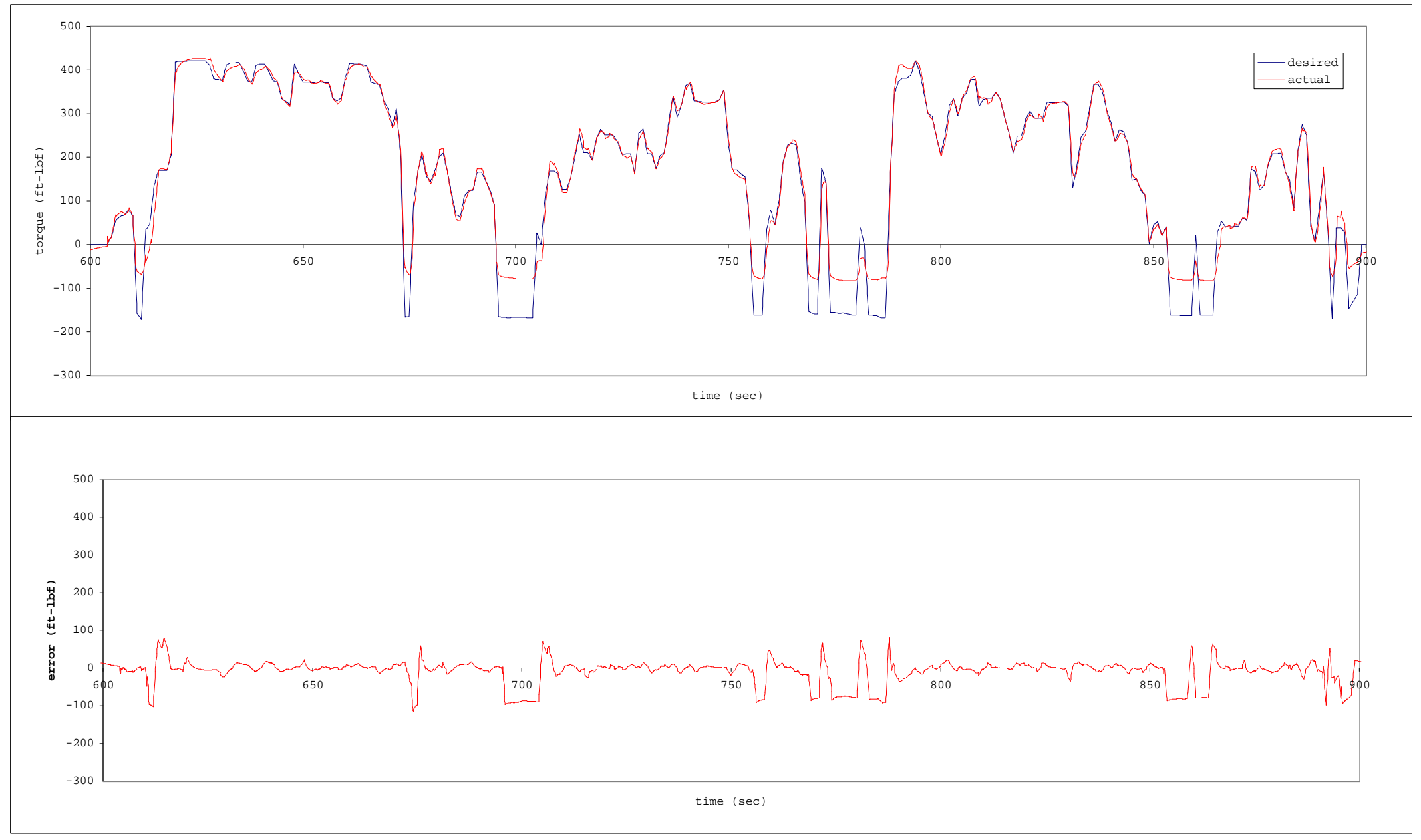

Figure 4.11b Desired and actual torque over the FTP cycle for NN, 0.01, control 


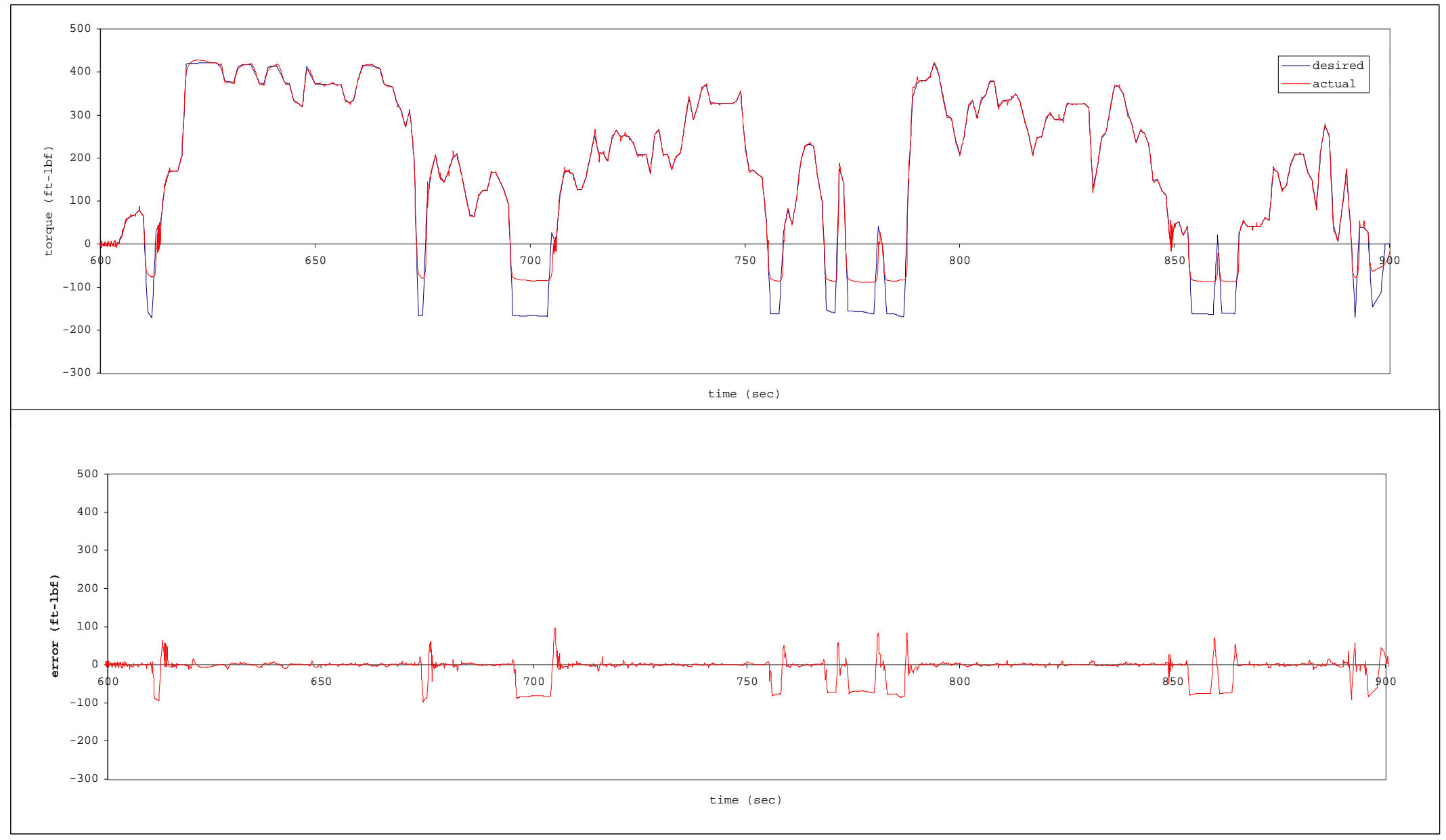

Figure 4.11c Desired and actual torque over the FTP cycle for NN, 0.10, control 


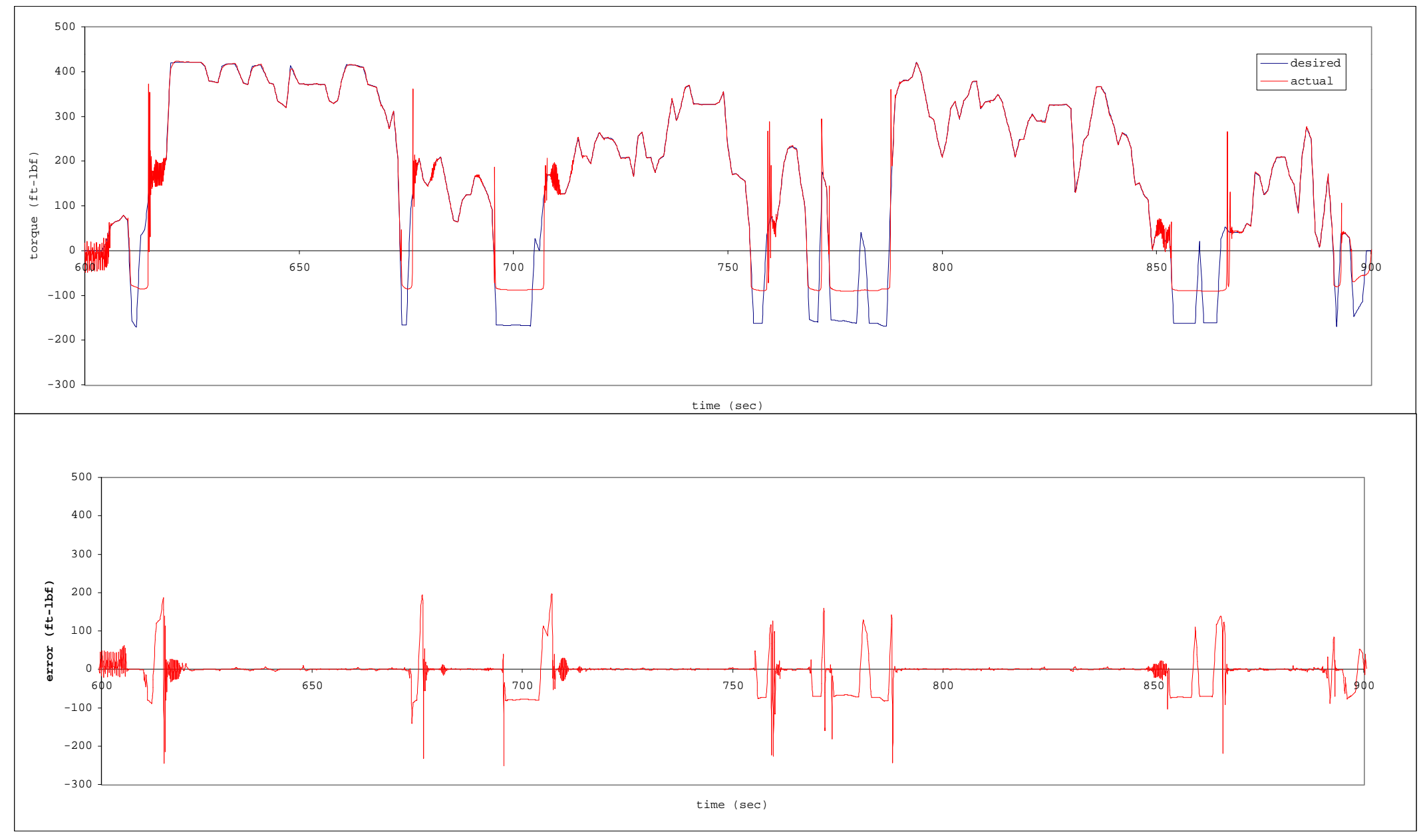

Figure 4.11d Desired and actual torque over the FTP cycle for NN, 1.0, control 


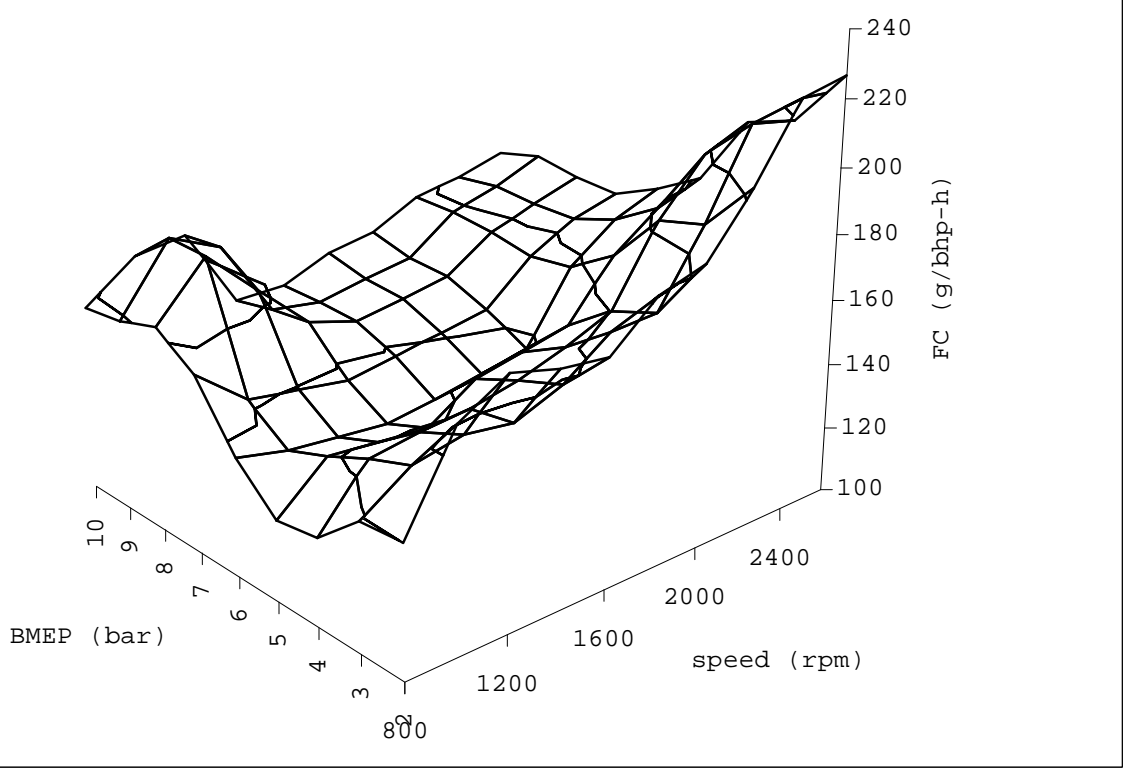

Figure 4.14a Brake specific fuel consumption at steady state operation

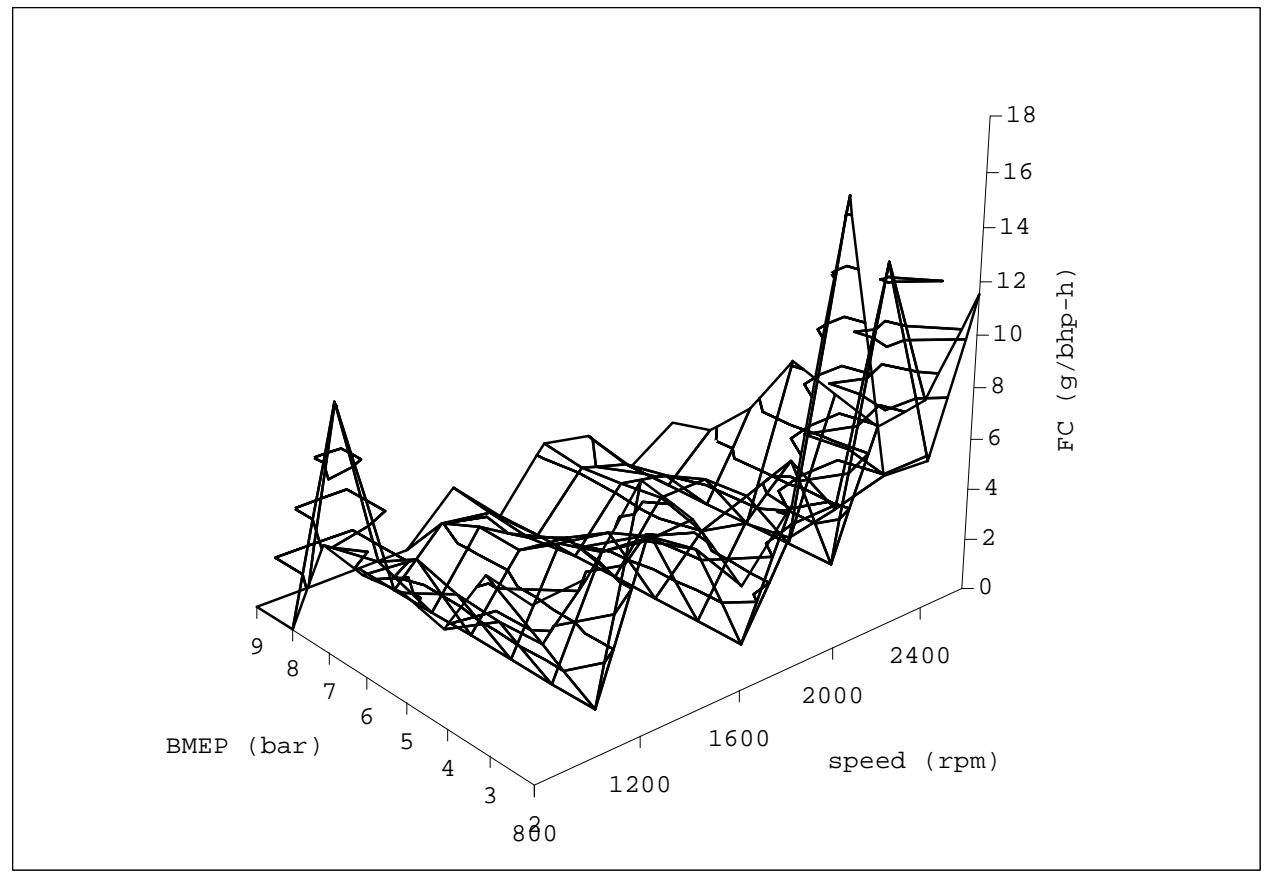

Figure 4.14b Standard deviation distribution of the fuel consumption map 


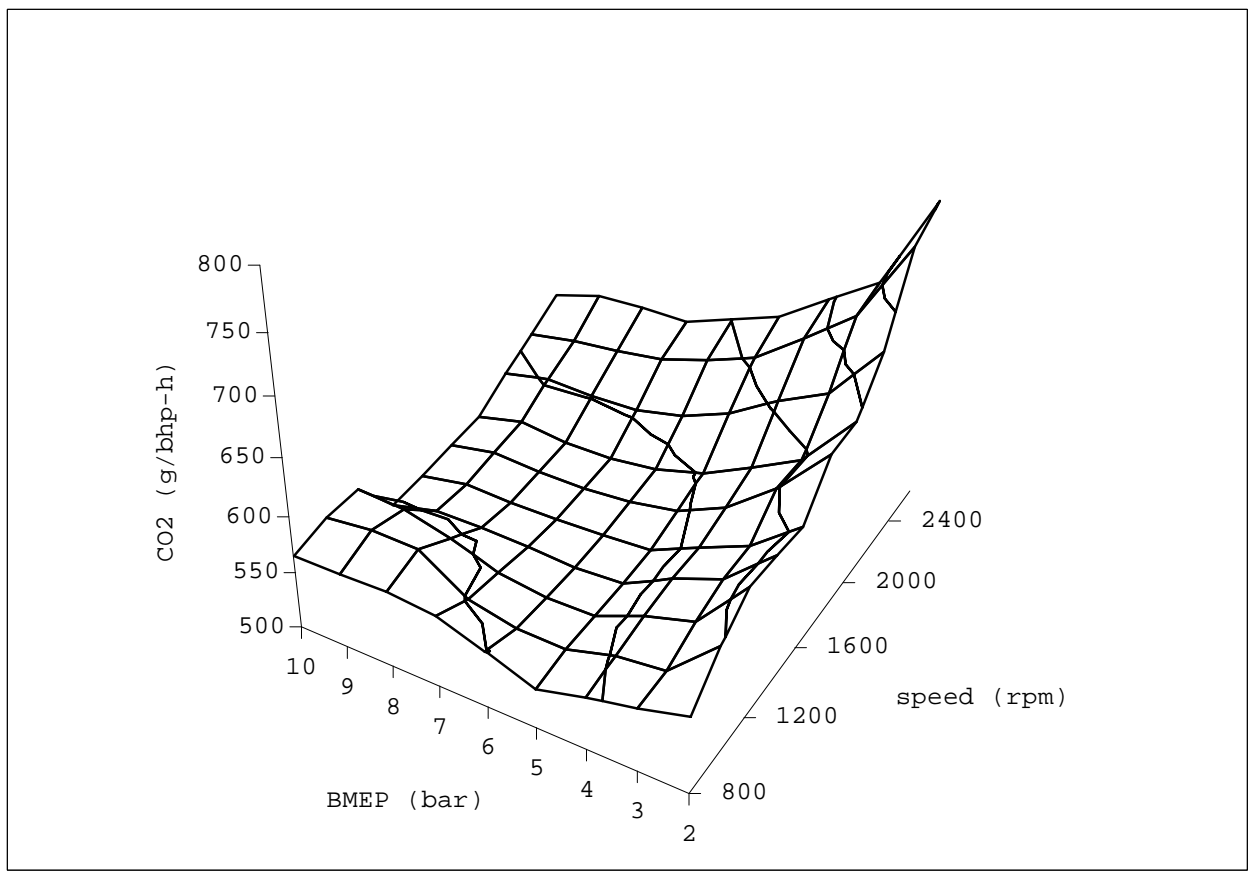

Figure 4.15a Brake specific CO2 emissions at steady state operation

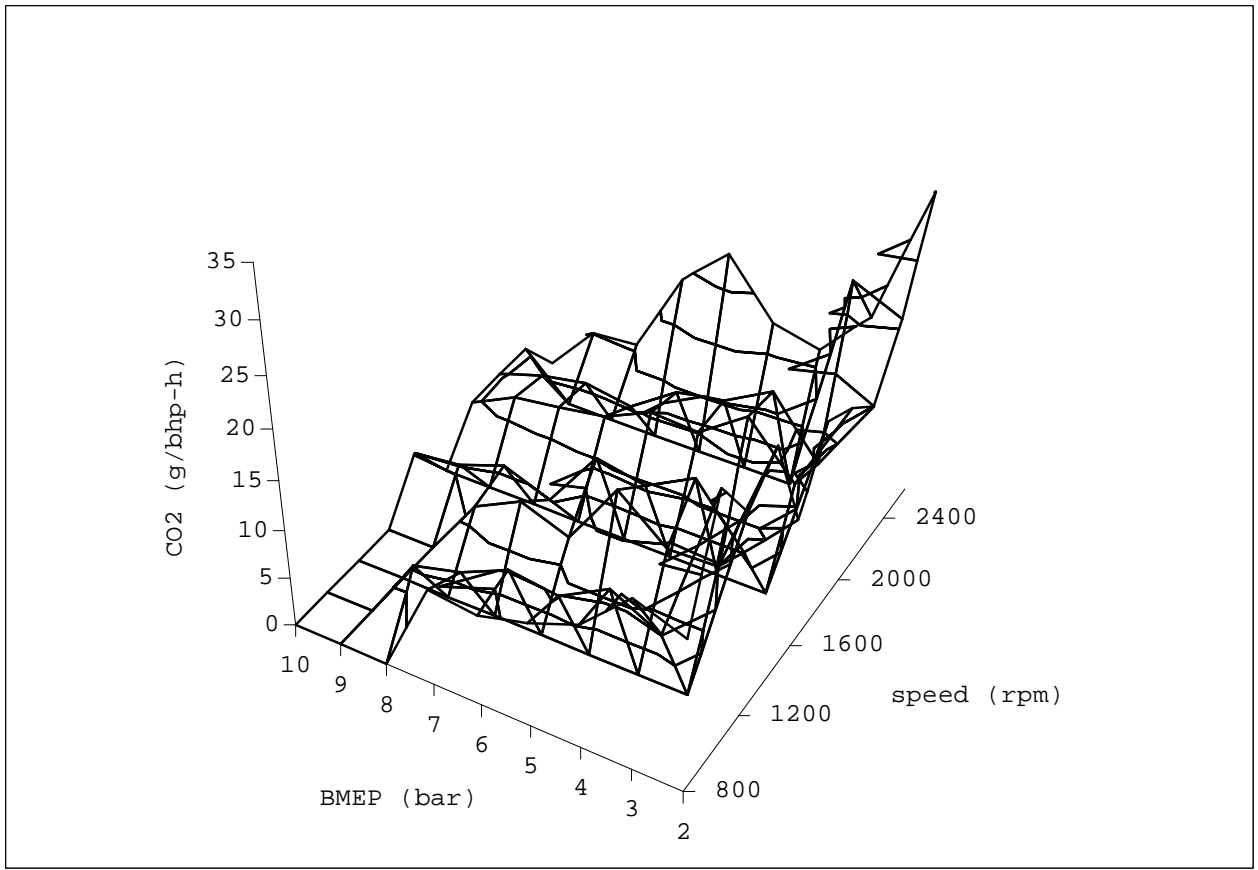

Figure 4.15b Standard deviation distribution of CO2 emissions map 


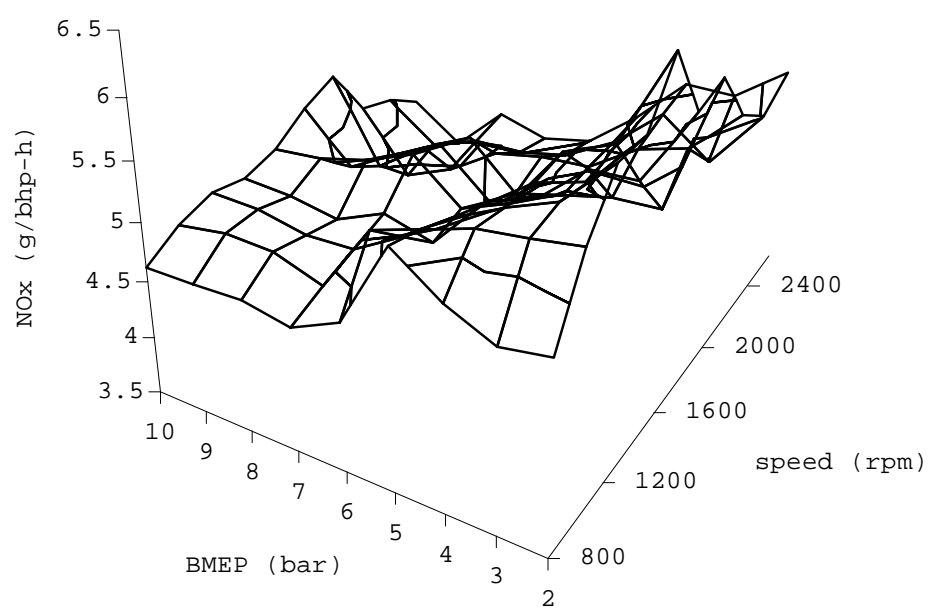

Figure 4.16a Brake specific NOx emissions at steady state operation

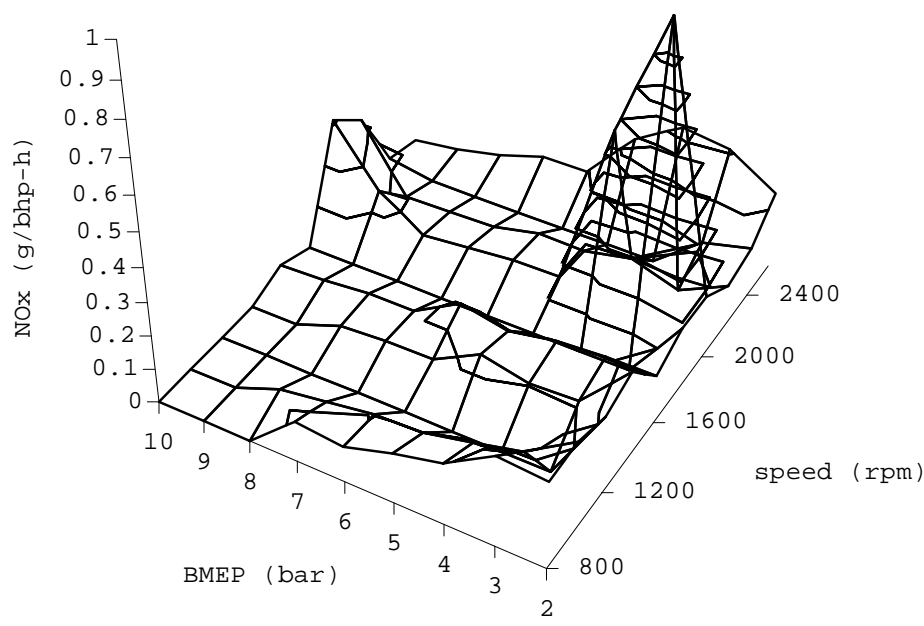

Figure 4.16b Standard deviation distribution of NOx emissions map 


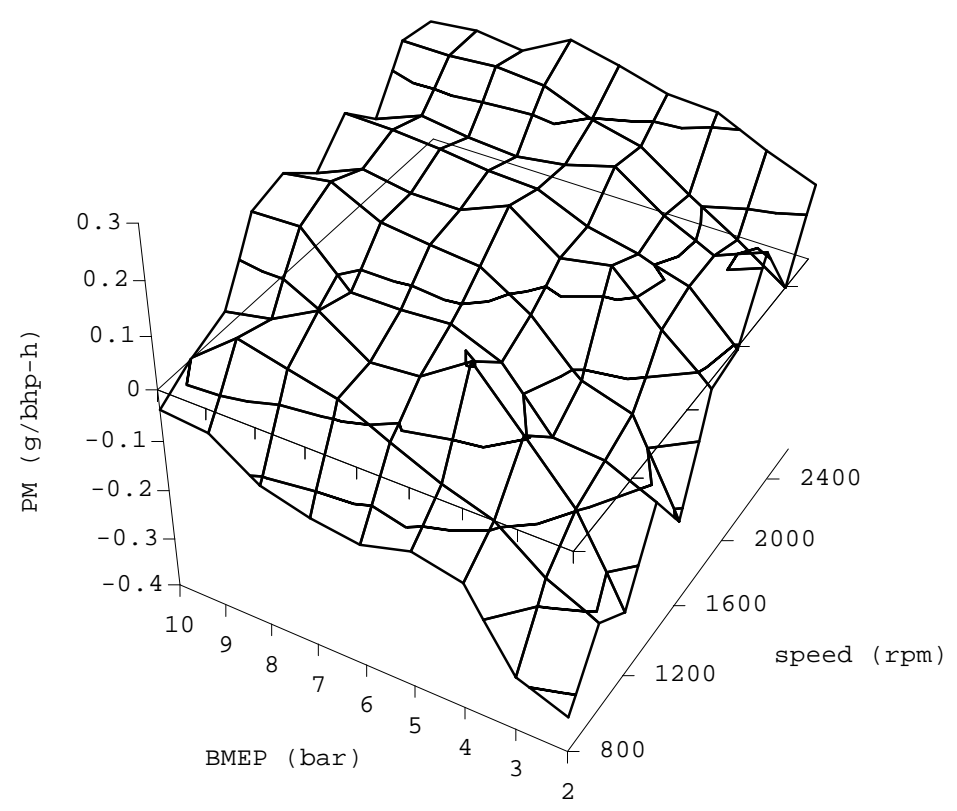

Figure 4.17a Brake specific PM emissions averaged over transient operation

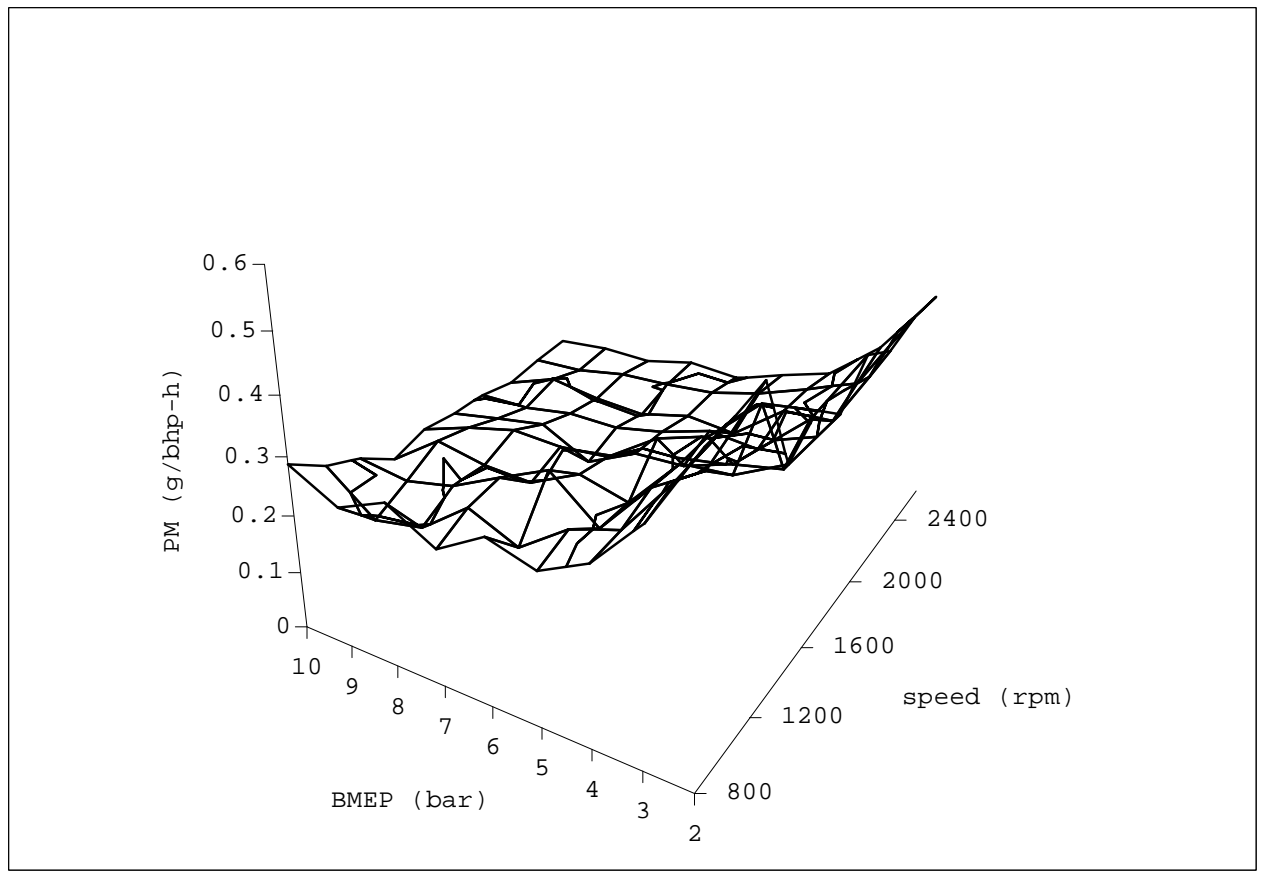

Figure 4.17b Standard deviation distribution of PM emissions map 


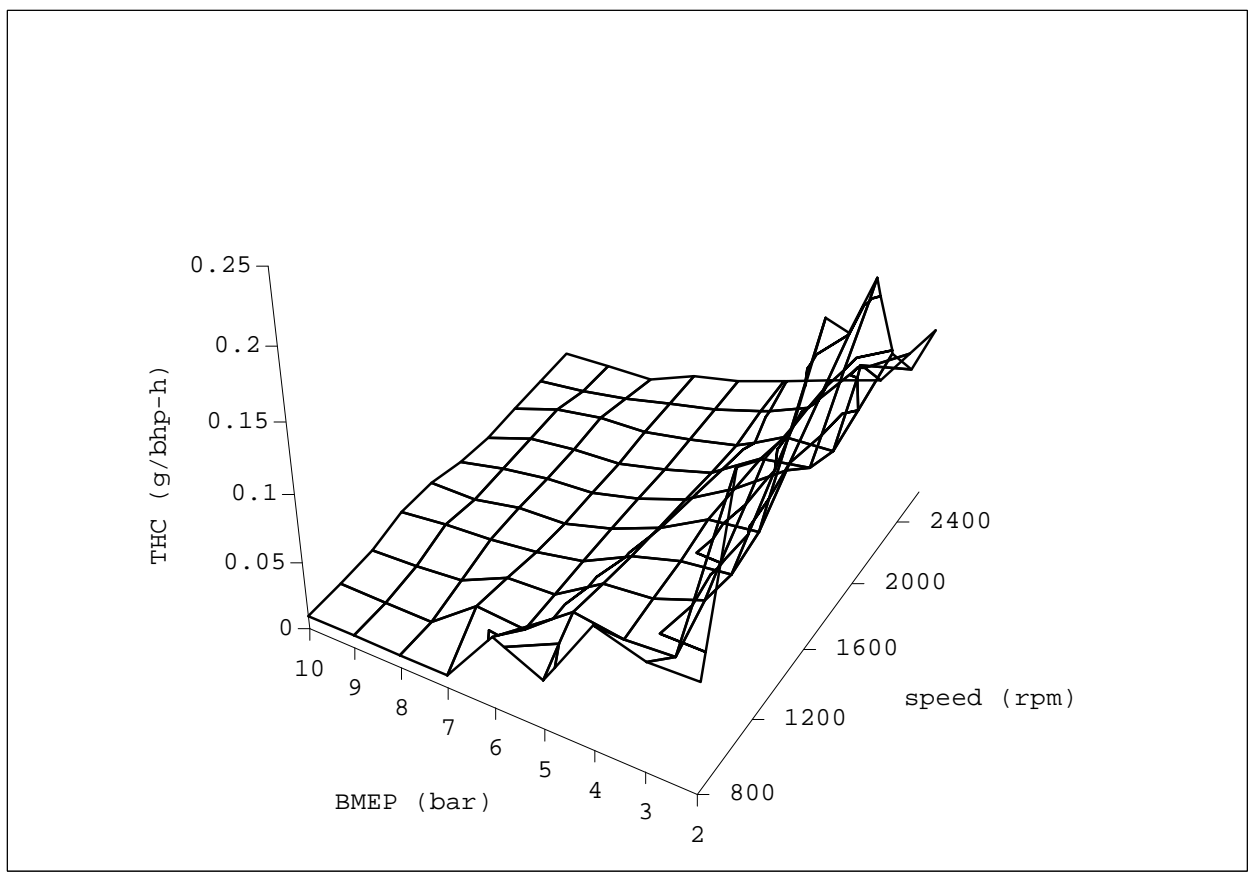

Figure 4.18a Brake specific THC emissions at steady state operation

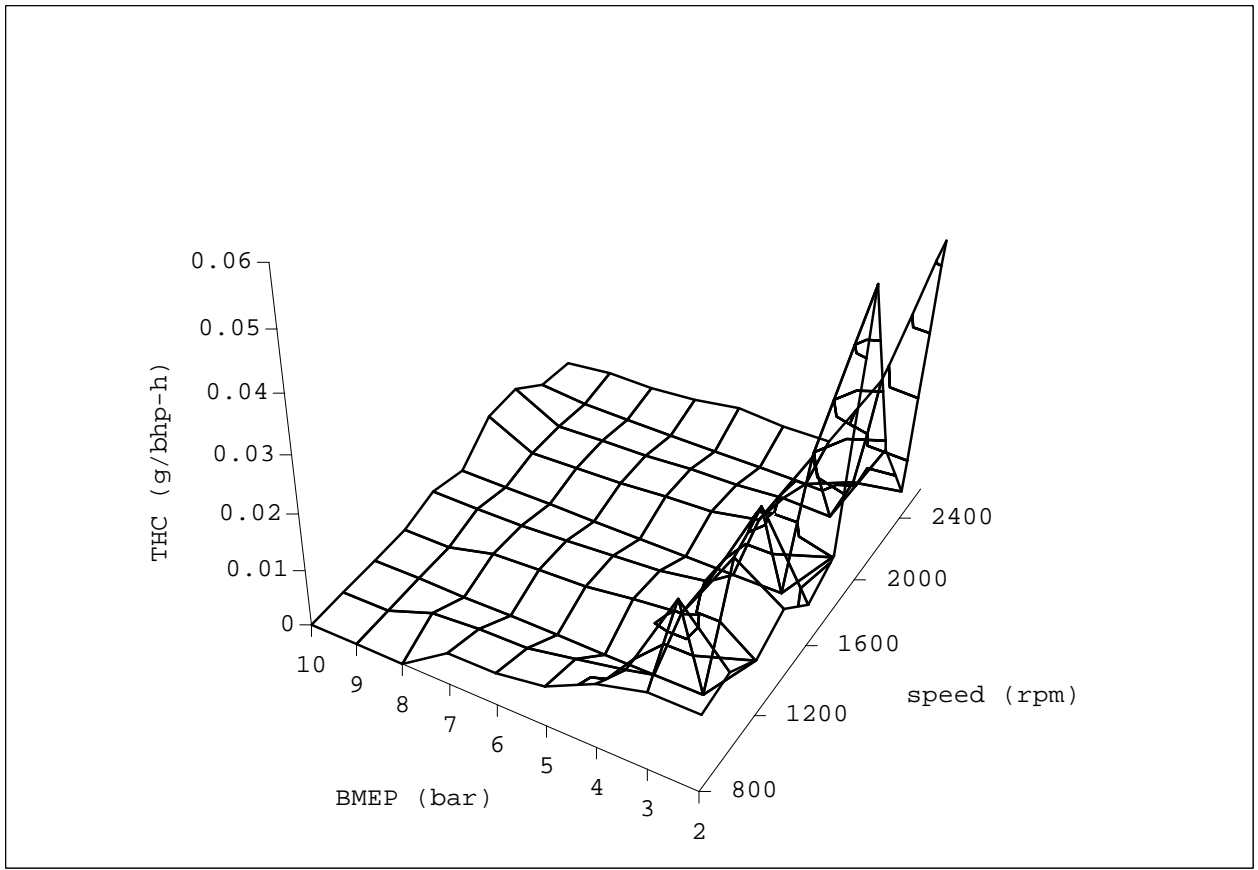

Figure 4.18b Standard deviation distribution of THC emissions map 


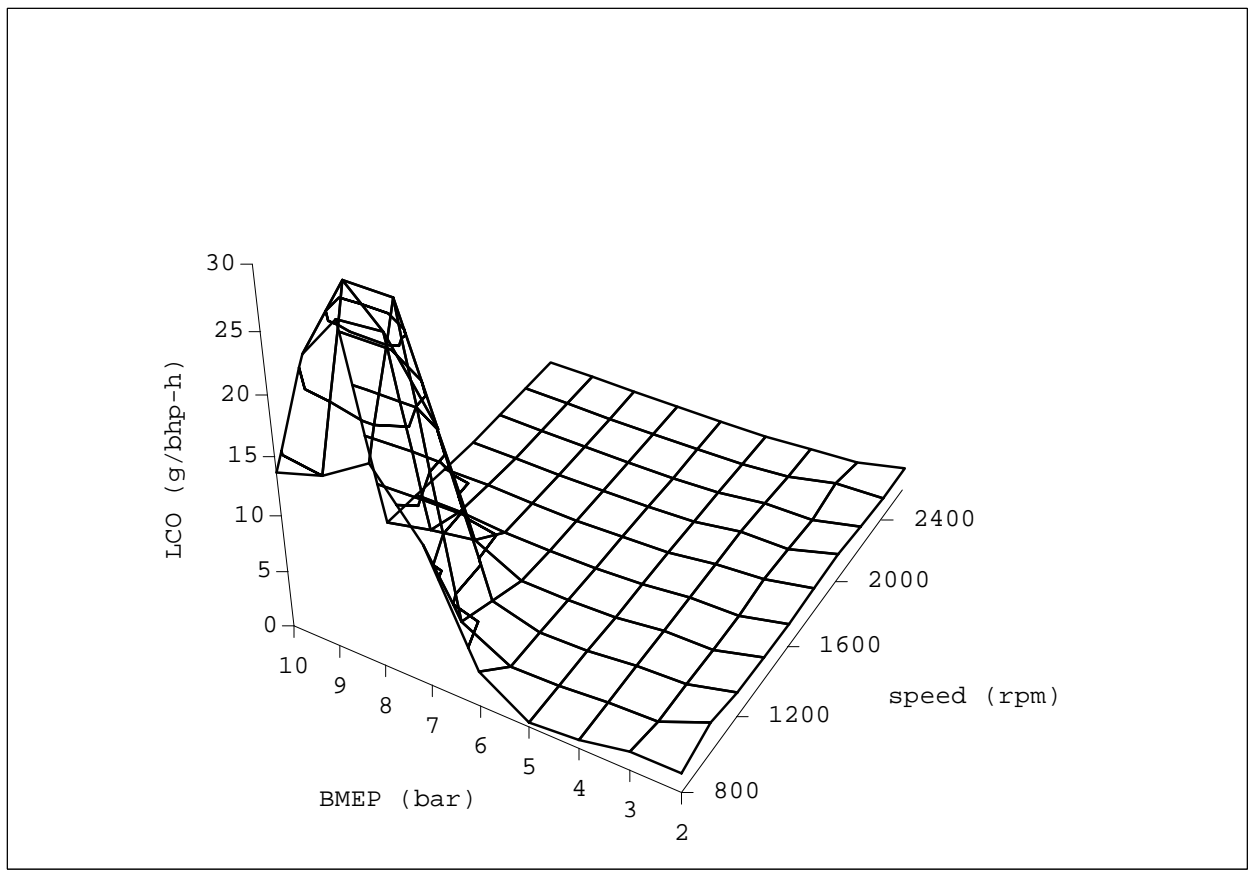

Figure 4.19a Brake specific LCO emissions at steady state operation

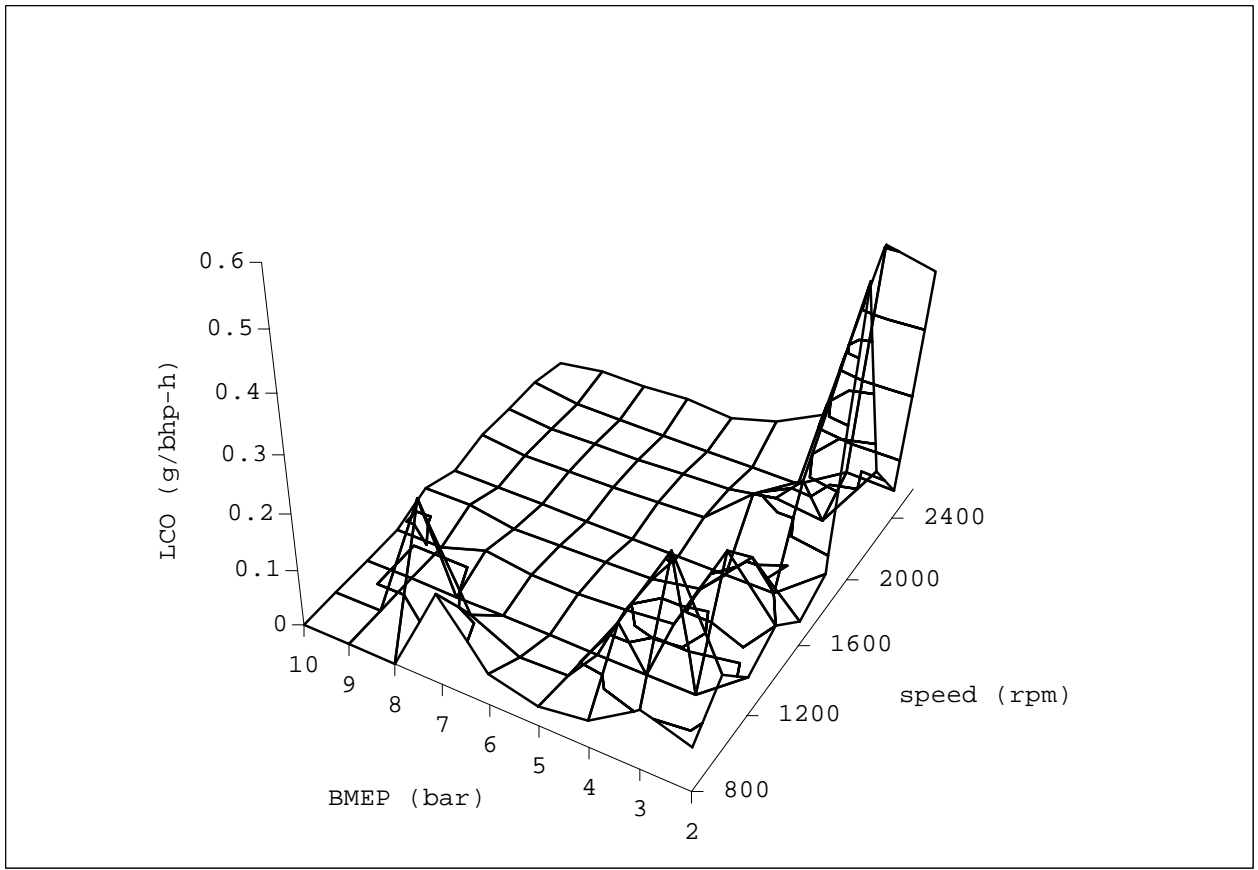

Figure 4.19b Standard deviation distribution of LCO emissions map 


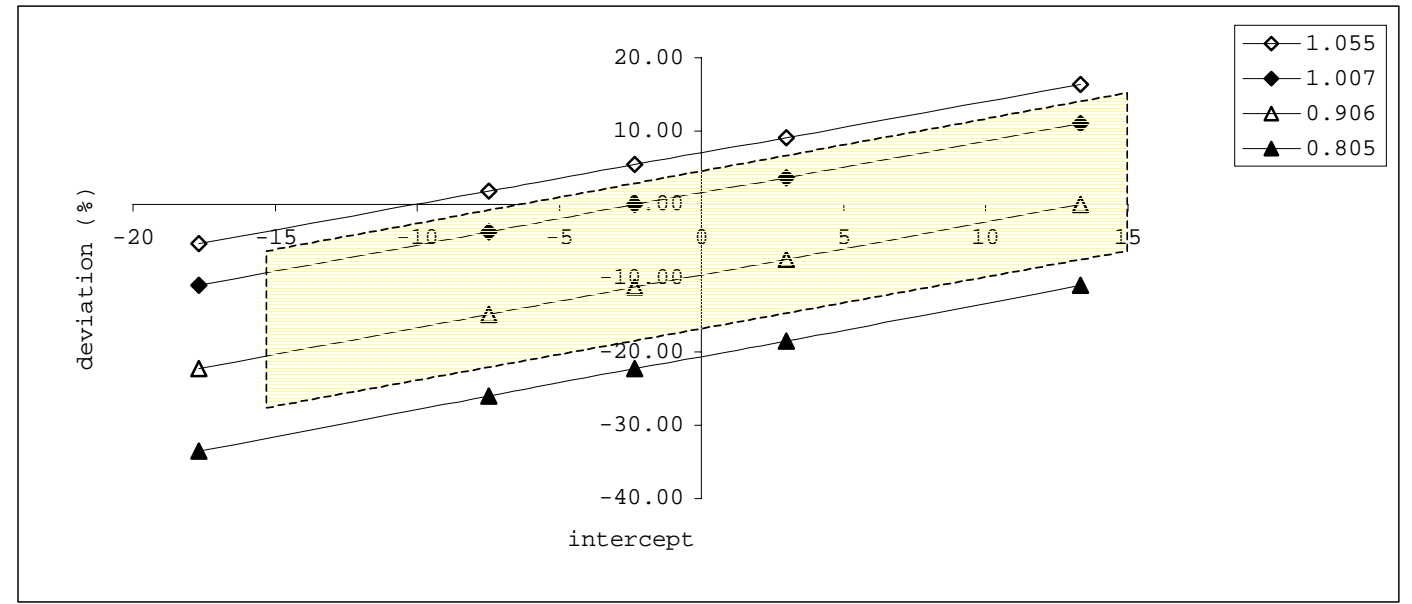

Figure 4.20a Total energy deviation versus regression slope and intercept over the FTP cycle

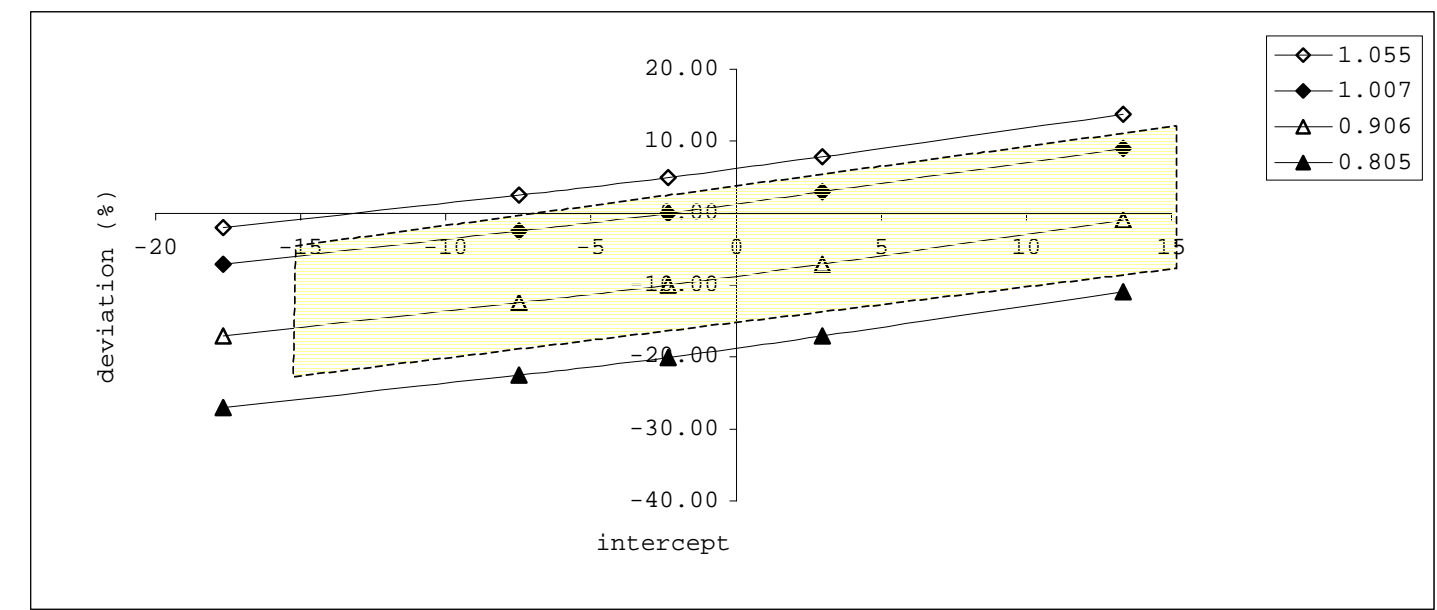

Figure 4.20b Positive energy deviation versus regression slope and intercept over the FTP cycle

EPA validation criteria for engine torque over the FTP test

\begin{tabular}{|l|c|c|c|c|c|}
\hline \multirow{2}{*}{ cycle } & \multicolumn{2}{|c|}{ error } & \multicolumn{3}{c|}{ regression } \\
\cline { 2 - 7 } & $\mathrm{e} 1$ & $\mathrm{e} 2\left(\frac{\circ}{\circ}\right)$ & $\mathrm{c}$ & $\mathrm{m}$ & r2 \\
\hline torque (ft-lbf) & 63.00 & 8.40 & -15 to +15 & 0.83 to 1.03 & 0.88 to 1.0 \\
\hline
\end{tabular}

N.B. Shaded area indicates allowable variation within the EPA validation criteria 


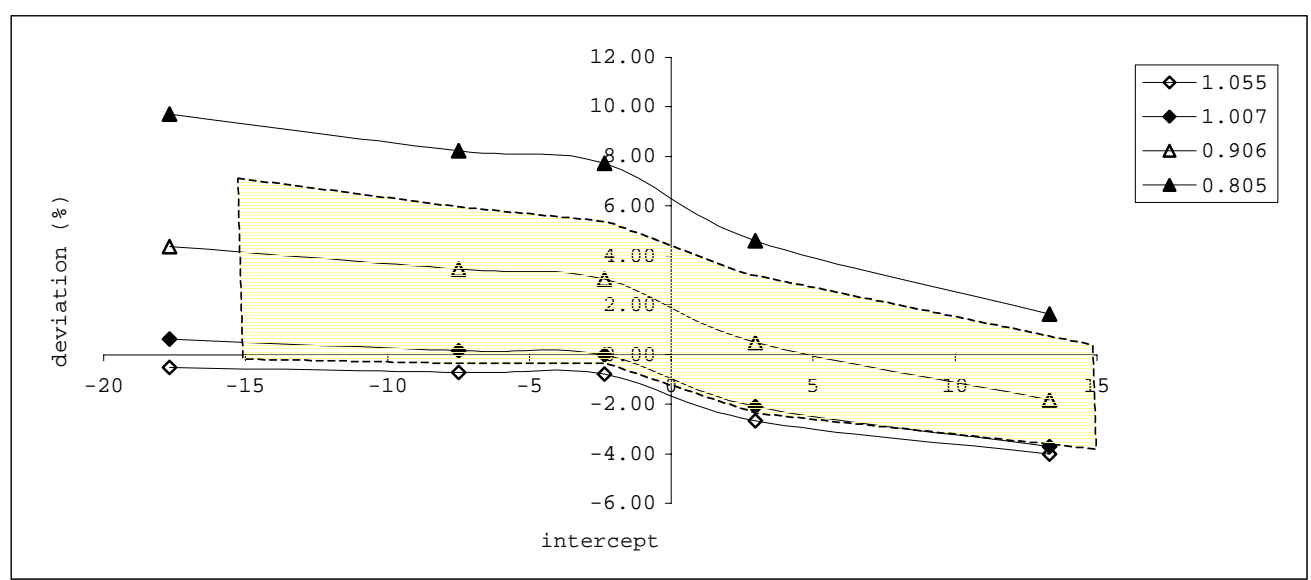

Figure 4.20c Brake specific FC deviation versus regression slope and intercept over the FTP cycle

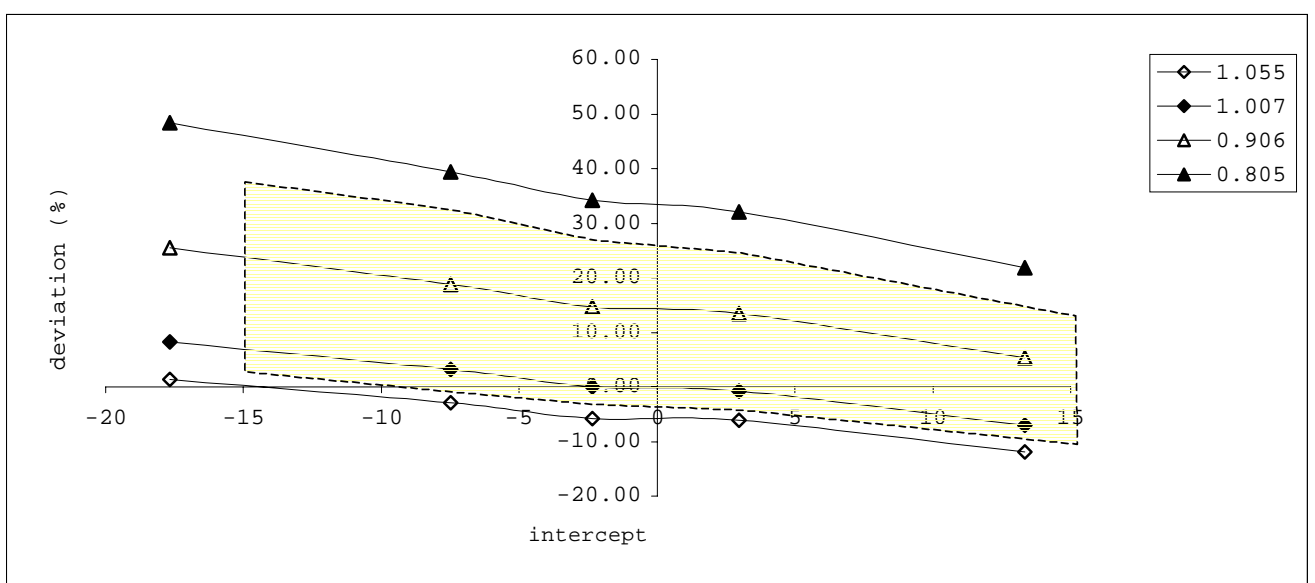

Figure 4.20d Brake specific THC emissions deviation versus regression slope and intercept over the FTP cycle

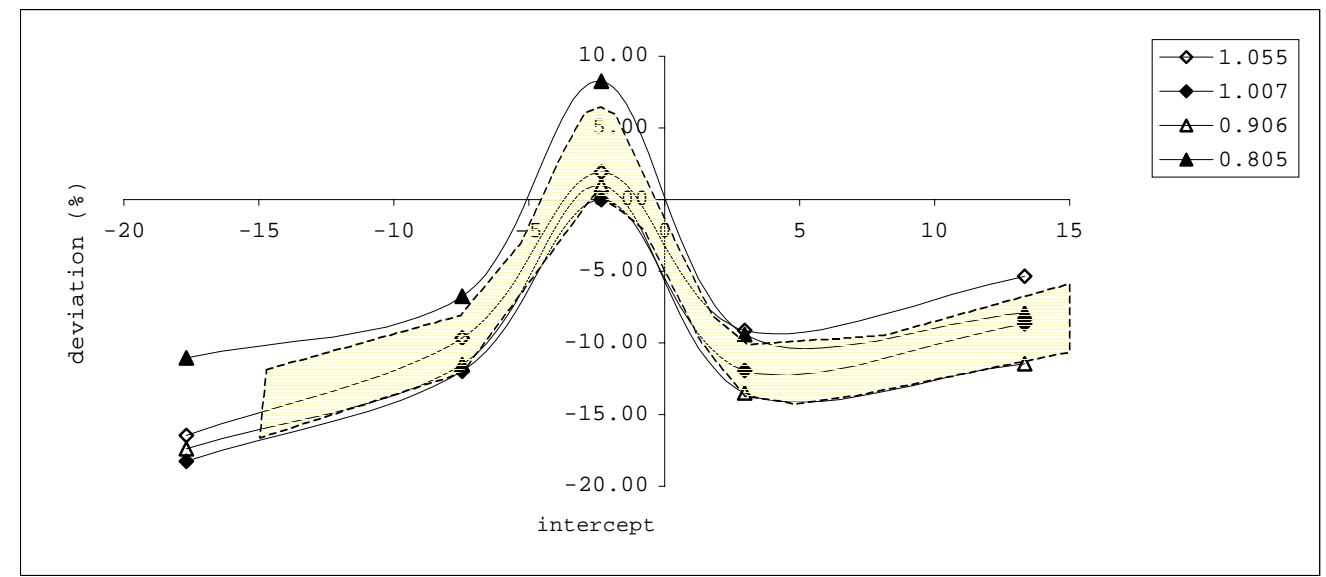

Figure 4.20e Brake specific LCO emissions deviation versus regression slope and intercept over the FTP cycle

N.B. Shaded area indicates allowable variation within the EPA validation criteria 

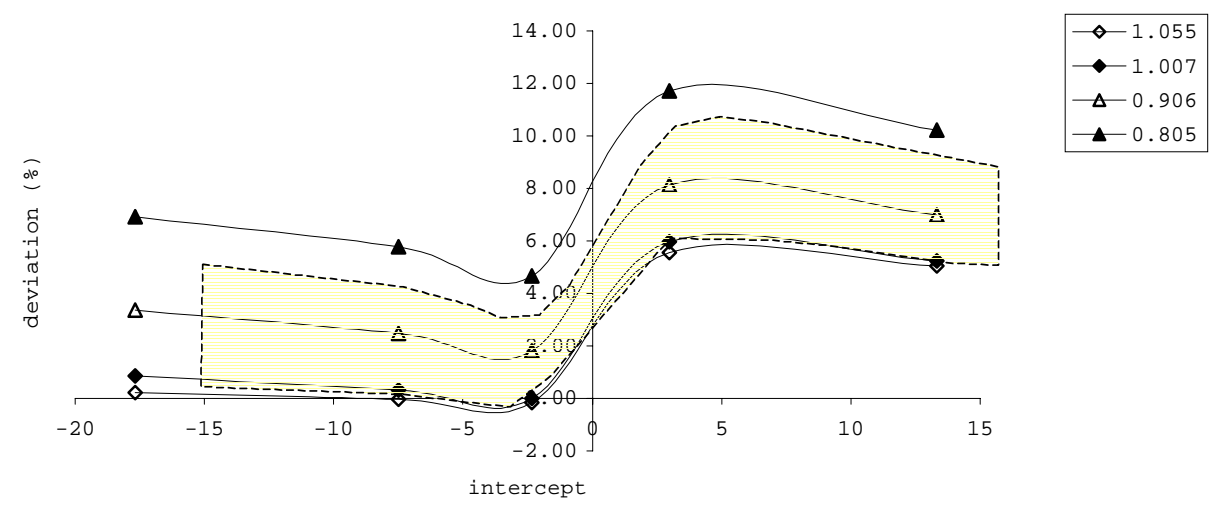

Figure 4.20f Brake specific CO2 emissions deviation versus regression slope and intercept over the FTP cycle

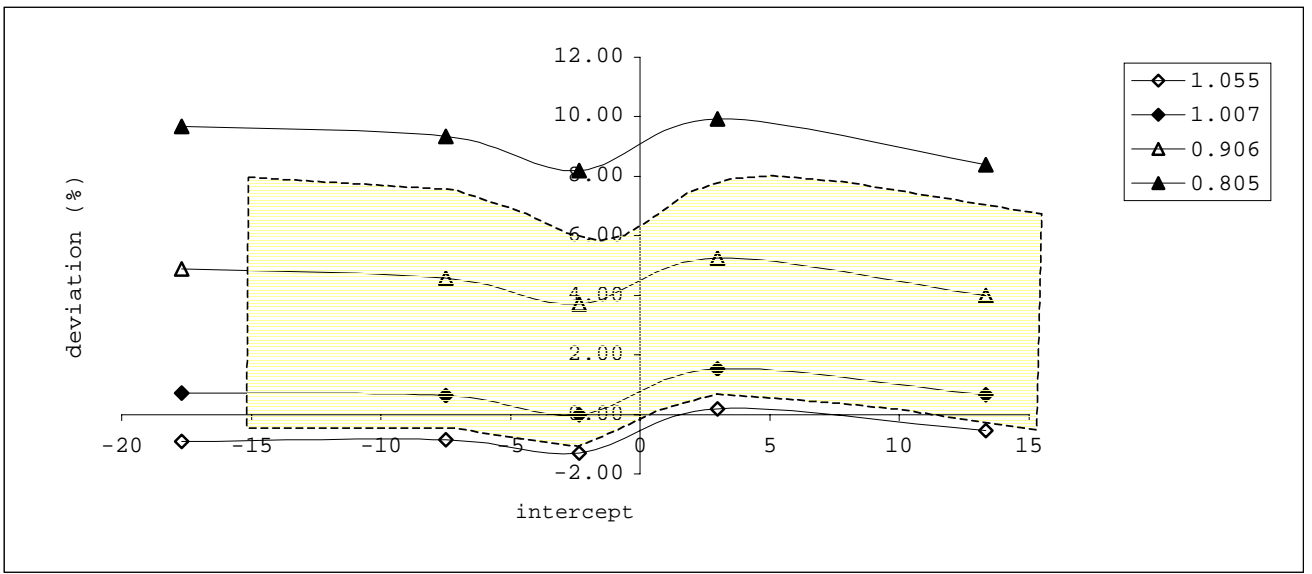

Figure 4.20g Brake specific NOx emissions deviation versus regression slope and intercept over the FTP cycle

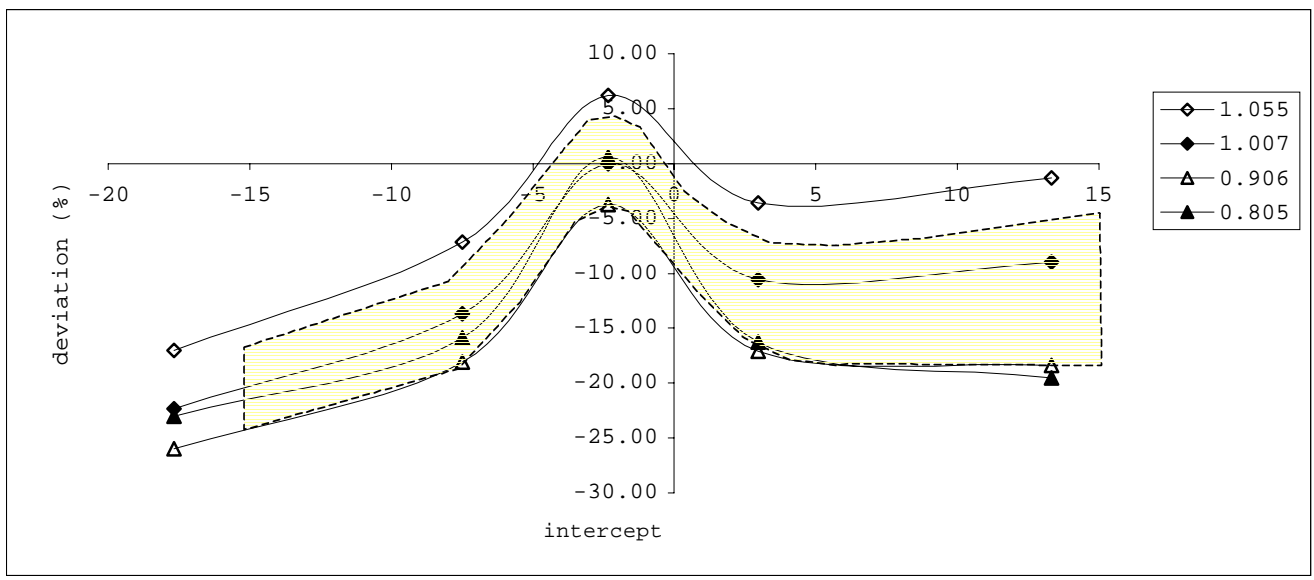

Figure 4.20h Brake specific PM emissions deviation versus regression slope and intercept over the FTP cycle

N.B. Shaded area indicates allowable variation within the EPA validation criteria 


\subsubsection{Governed Engine Operation}

The sub-components of the torque controller for the case of governed engine operation are shown in Figure 4.21.

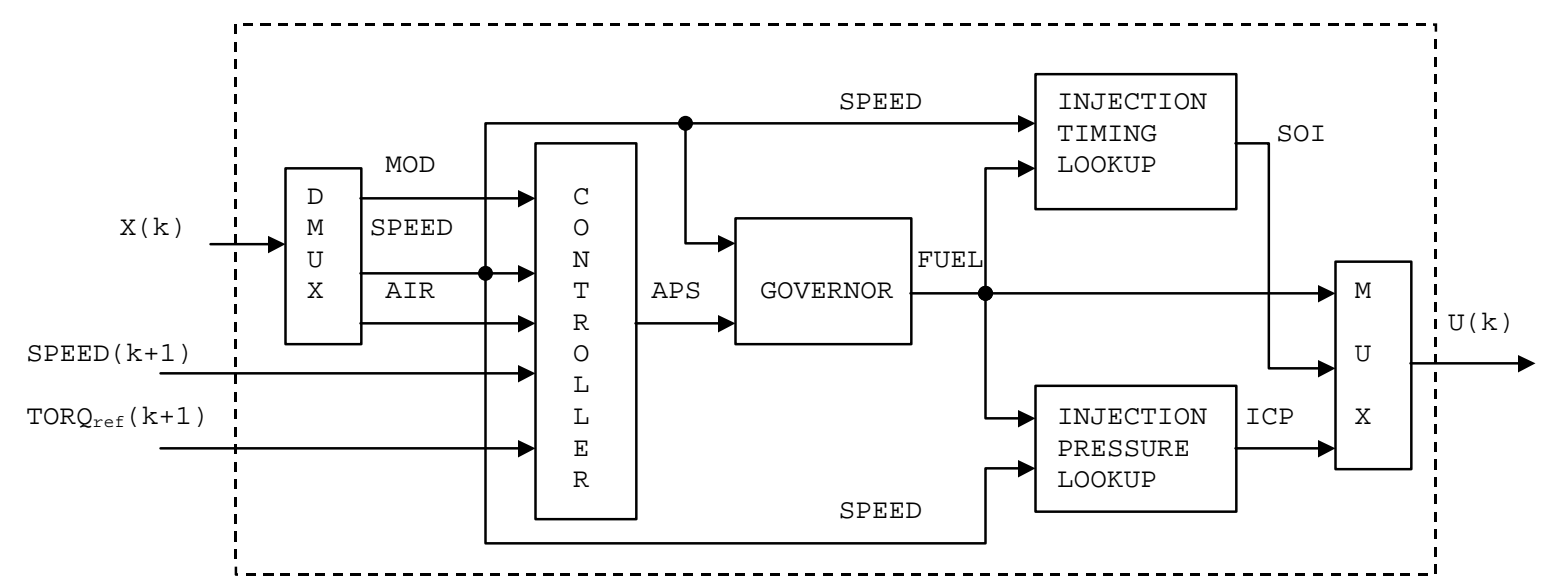

Figure 4.21 Schematic of the torque controller for governed engine operation

The output of the NN controller is the accelerator position signal (APS), which is passed to the governor lookup table along with engine speed in order to determine the fuel flow rate. As discussed in the previous section, the injection timing and pressure are looked up using engine speed and the commanded fuel flow rate.

Two types of proportional governors have been considered where type II implements a more aggressive fueling strategy compared to governor type I, as shown in Figure 4.22a. A stepped accelerator test cycle was simulated for both governors in order to estimate the engine performance and exhaust gas emissions characteristics. For a given engine speed, the accelerator position was stepped at an increment of 5\% in the range from $0 \%$ to $100 \%$, as shown in Figure 4.22b. The engine speed was varied from $700 \mathrm{rpm}$ to $2220 \mathrm{rpm}$ at an increment of $380 \mathrm{rpm}$. The estimated engine torque characteristics for both governors are shown in Figure 4.23, where a hysteretic effect was demonstrated for the transient operation and the rise in torque was steeper 
for governor type II. The uneven change in torque is attributed to the large step changes in APS signal, which was set at $5 \%$, within an active range of approximately $10 \%$ to $20 \%$ depending on the type of governor used. The predicted emissions are shown in Figure 4.24. No differences were predicted in $\mathrm{CO}_{2}$ and NOx emissions between the two governors. The excursions in PM emissions was higher for the type II governor compared to the less aggressive type I. The expected spikes in LCO and THC emissions were predicted for the sharp transient operation, as shown in Figures 4.24d and 4.24e. Over-fueling at high load transient was higher for type II compared to governor I because of its more aggressive nature, as shown in Figure 4.24f.

For the purposes of closed-loop torque control simulations, the governed fueling flow rate lookup table was modeled using a four neuron single hidden layer NN with non-linear activation in both layers. The NNs for governors I and II were trained in a batch mode using the Levenberg-Marquardt algorithm in the MATLAB environment. The governor NN was inserted into the integral network used for the non-governed engine operation, as shown in Figure 4.25. The formed network consisted of ten layers, which were not fully connected. In a manner similar to that described in the previous section, the integral network was connected to the forward dynamics NN and emissions NNs in order to form the closed-loop simulation for the governed engine operation. The NN controller was adapted using the standard back-propagation algorithm and all other NNs were frozen at the values acquired through offline training.

In order to guarantee initial stability, the NN controller was trained offline, in a batch mode, to mimic the behavior of a PI controller and the predicted torque value at the next time step was used as the current step desired value. The tracking performance with the PI control for the governed operation is shown in Figure 4.26. The simulated test cycle consisted of four consecutive torque pulses (fractions of $0.20,0.40,0.60$, and 0.80 of maximum torque) at a 
constant engine speed, which spanned a range from $700 \mathrm{rpm}$ to $2400 \mathrm{rpm}$ at a step of $340 \mathrm{rpm}$. The good tracking performance of the PI control, for both governors, provided an excellent initial start for the NN control, which was later adapted online.

The closed-loop simulation was conducted for the FTP test cycle for the PI controller as well as the NN controller and the two governors. For governor type I, the NN controller learning parameter value was $\lambda=0.005$, which was reduced to $\lambda=0.001$ for the more aggressive governor type II as it required finer tuning of the APS signal.

The torque tracking performance over the FTP for the PI and NN controllers is shown in Figures 4.27 and 4.28. The NN-based controllers demonstrate excellent tracking performance compared to the slightly damped performance achieved using the PI control, which suffers apparent lag in the rise in torque at sharp transients. This is further confirmed through the error analysis of the positive torque tracking, shown in Table 4.9a.

Table 4.9a Tracking analysis of positive torque over the FTP for governed operation

\begin{tabular}{|l|r|r|r|r|}
\hline \multirow{2}{*}{ Measure } & \multicolumn{2}{|c|}{ governor I } & \multicolumn{2}{c|}{ governor II } \\
\cline { 2 - 5 } & PI & NN & PI & NN \\
\hline $\mathrm{e} 1$ (ft-lbf) & 51.85 & 13.51 & 51.76 & 10.98 \\
\hline $\mathrm{e} 2$ (\%) & 4.51 & 1.17 & 4.50 & 0.95 \\
\hline $\mathrm{e} 3\left(\frac{\circ}{\circ}\right)$ & 12.76 & -0.18 & 12.04 & 0.44 \\
\hline $\mathrm{c}(\mathrm{ft}-\mathrm{lbf})$ & -5.99 & 0.32 & -6.46 & -0.66 \\
\hline $\mathrm{m}(\mathrm{n} / \mathrm{a})$ & 0.92525 & 0.99899 & 0.93659 & 1.00146 \\
\hline $\mathrm{r} 2(\mathrm{n} / \mathrm{a})$ & 0.86959 & 0.99021 & 0.87033 & 0.99356 \\
\hline
\end{tabular}

The integral results for the different controllers are shown in Table 4.9b. For the NN controllers, the total and positive expended energy was higher and FC and THC emissions were slightly lower compared to the results achieved using PI control.

The $\mathrm{CO}_{2}$ and NOx emissions are slightly higher for the case of NN control independent of the type of governor used. Using NN control, PM emissions were slightly lower for governor I and higher for governor II, which is to be expected, noting that type I governor displayed a less aggressive fueling strategy. 
Table 4.9b Integral results over the FTP for governed operation

\begin{tabular}{|l|r|r|r|r|}
\hline \multirow{2}{*}{ parameter } & \multicolumn{2}{|c|}{ governor I } & \multicolumn{2}{c|}{ governor I } \\
\cline { 2 - 5 } & \multicolumn{1}{|c|}{$\mathrm{PI}$} & \multicolumn{1}{c|}{$\mathrm{NN}$} & \multicolumn{1}{c|}{$\mathrm{PI}$} & \multicolumn{1}{c|}{ NN } \\
\hline ENERGY $(\mathrm{bhp}-\mathrm{h})$ & 8.993 & 10.078 & 8.985 & 10.016 \\
\hline ENERGY+ (bhp-h) & 10.623 & 11.397 & 10.674 & 11.397 \\
\hline $\mathrm{FC}(\mathrm{g} / \mathrm{bhp}-\mathrm{h})$ & 189.130 & 187.762 & 188.791 & 186.783 \\
\hline $\mathrm{THC}(\mathrm{g} / \mathrm{bhp}-\mathrm{h})$ & 0.207 & 0.198 & 0.204 & 0.194 \\
\hline $\mathrm{LCO}(\mathrm{g} / \mathrm{bhp}-\mathrm{h})$ & 1.764 & 1.970 & 1.792 & 1.927 \\
\hline $\mathrm{CO}_{2}(\mathrm{~g} / \mathrm{bhp}-\mathrm{h})$ & 640.557 & 653.476 & 640.997 & 643.956 \\
\hline $\mathrm{NOx}(\mathrm{g} / \mathrm{bhp}-\mathrm{h})$ & 5.463 & 5.508 & 5.451 & 5.465 \\
\hline $\mathrm{PM}(\mathrm{g} / \mathrm{bhp}-\mathrm{h})$ & 0.090 & 0.088 & 0.090 & 0.091 \\
\hline
\end{tabular}




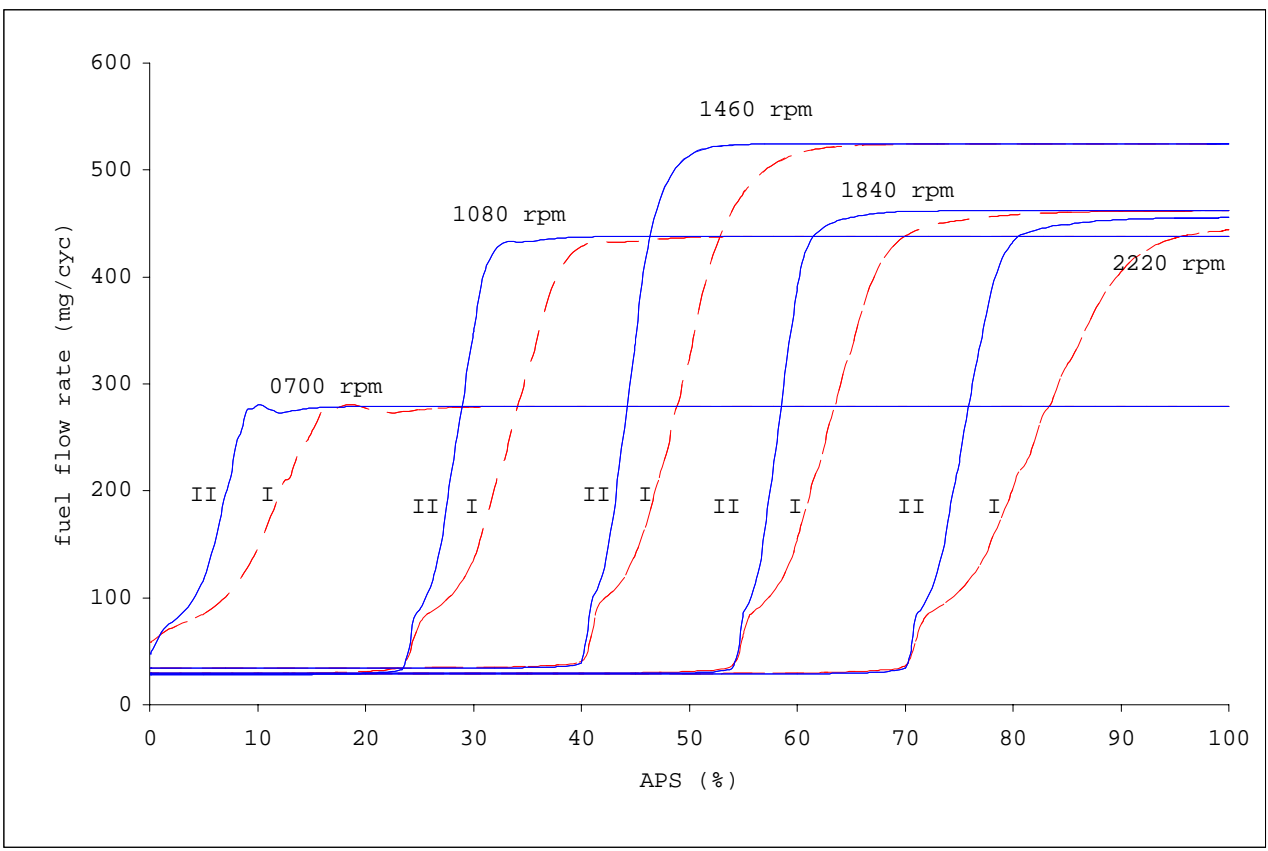

Figure 4.22a Fuel flow rate characteristics for the two types of proportional governors considered

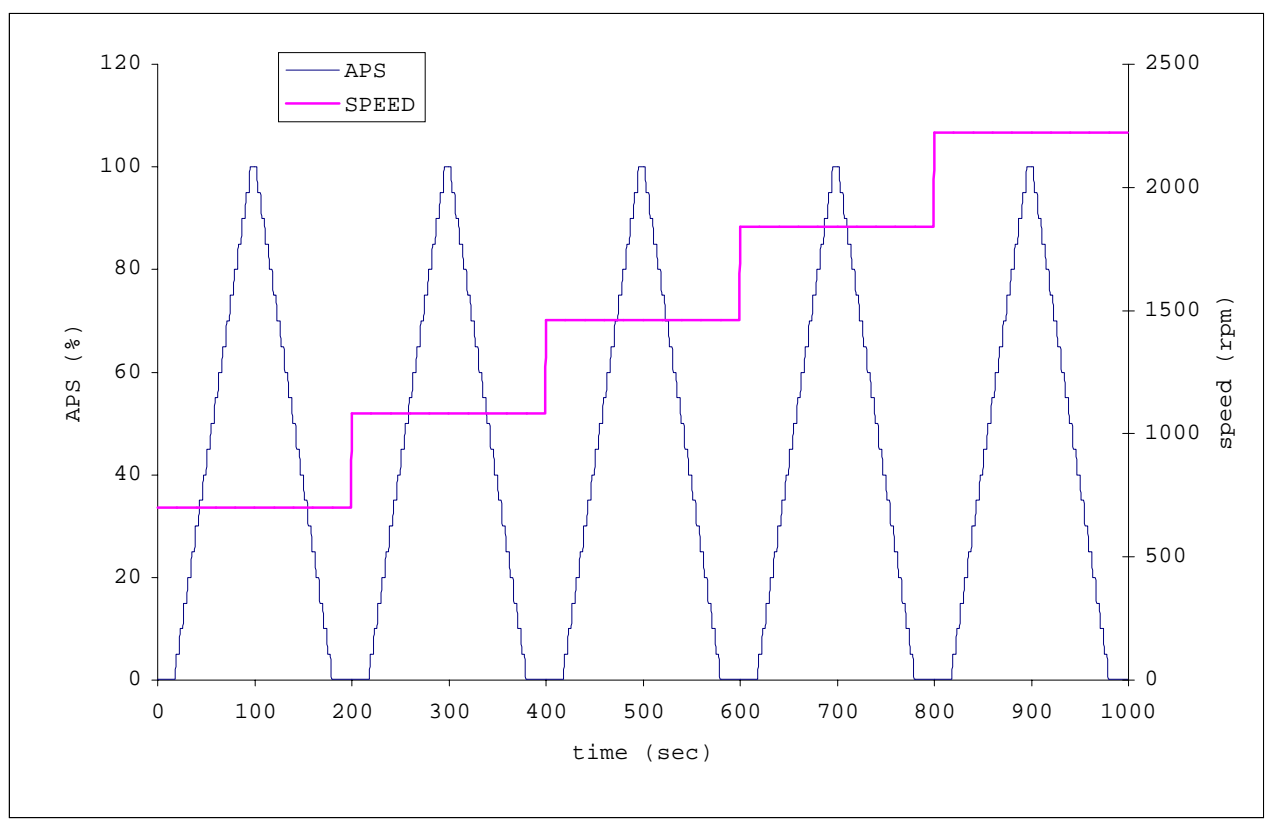

Figure 4.22b Stepped accelerator position cycle at different engine speeds 


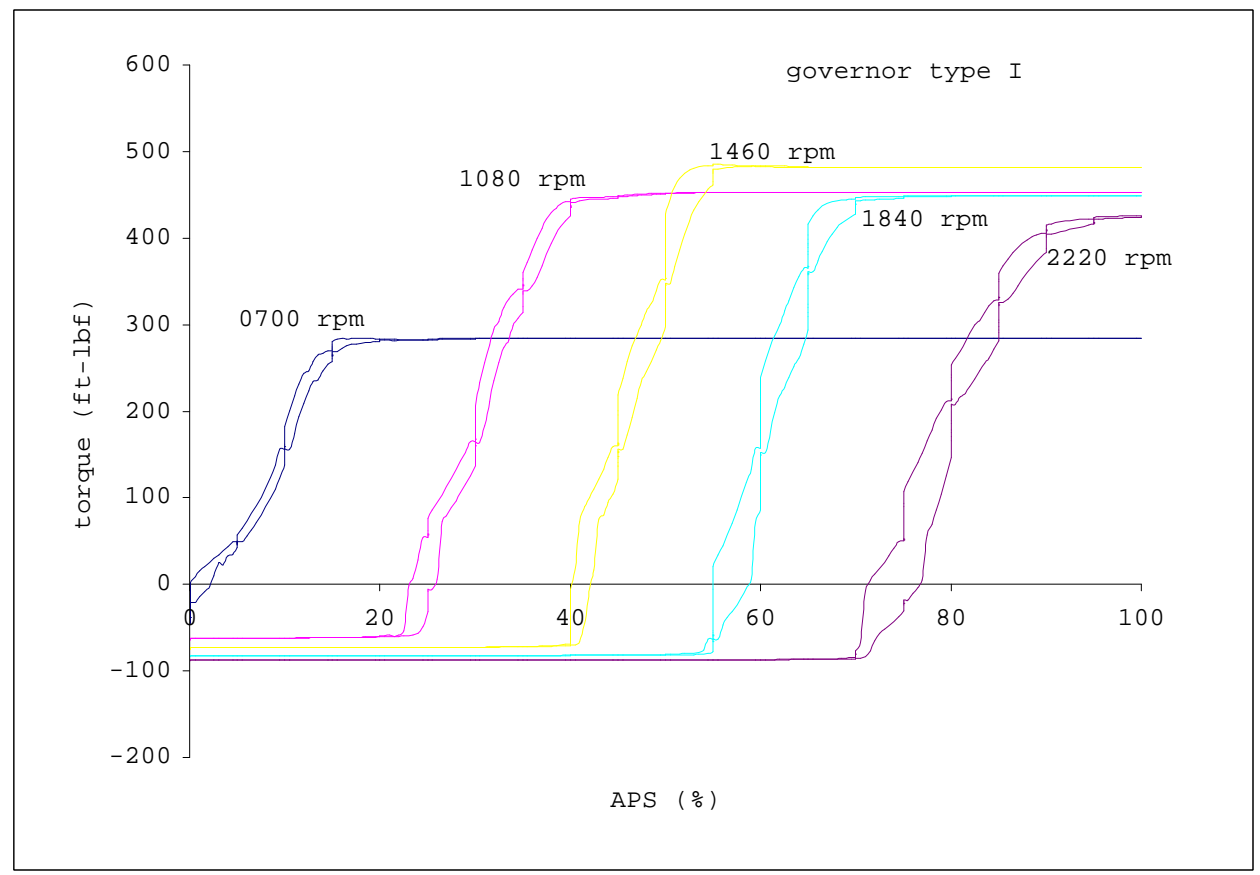

Figure 4.23a Torque rise characteristics for proportional governor type I

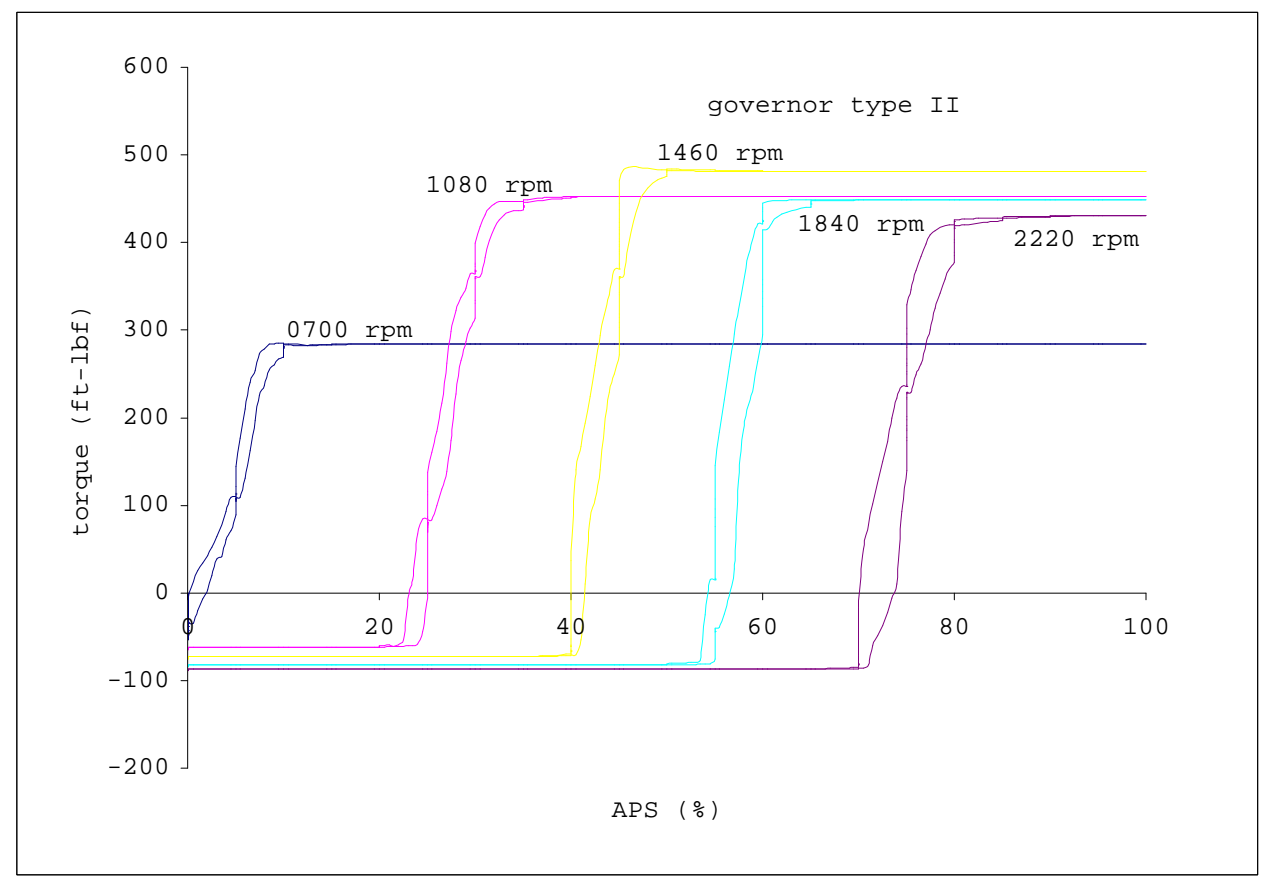

Figure 4.23b Torque rise characteristics for proportional governor type II 


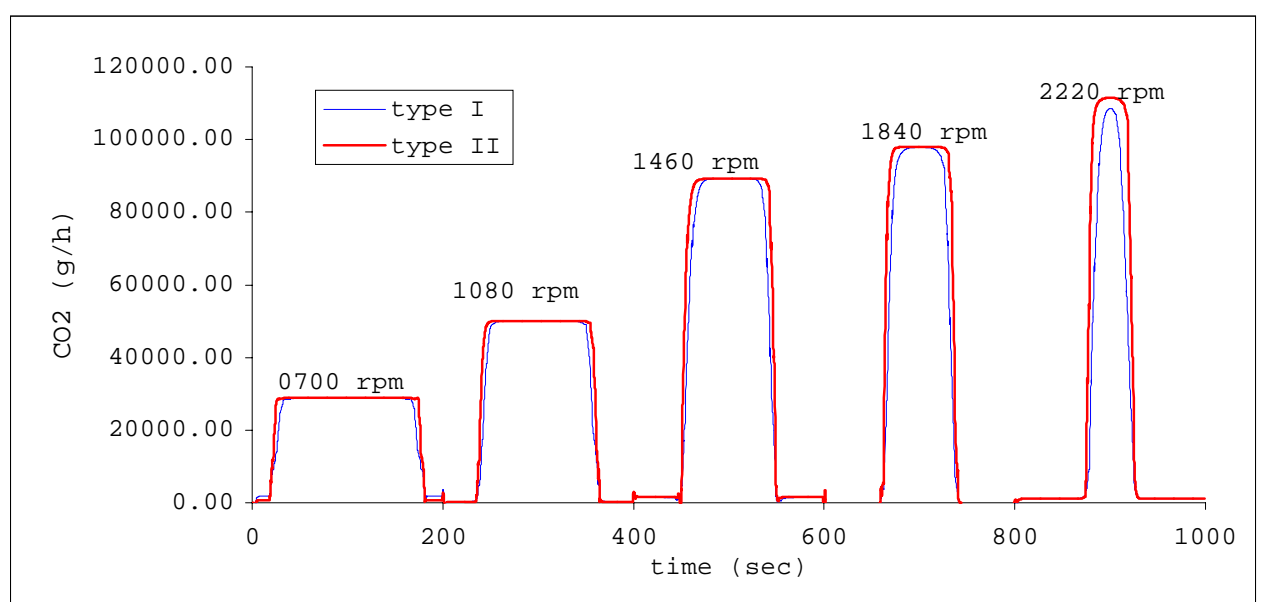

Figure 4.24a Carbon dioxide emissions for the two types of proportional governors considered

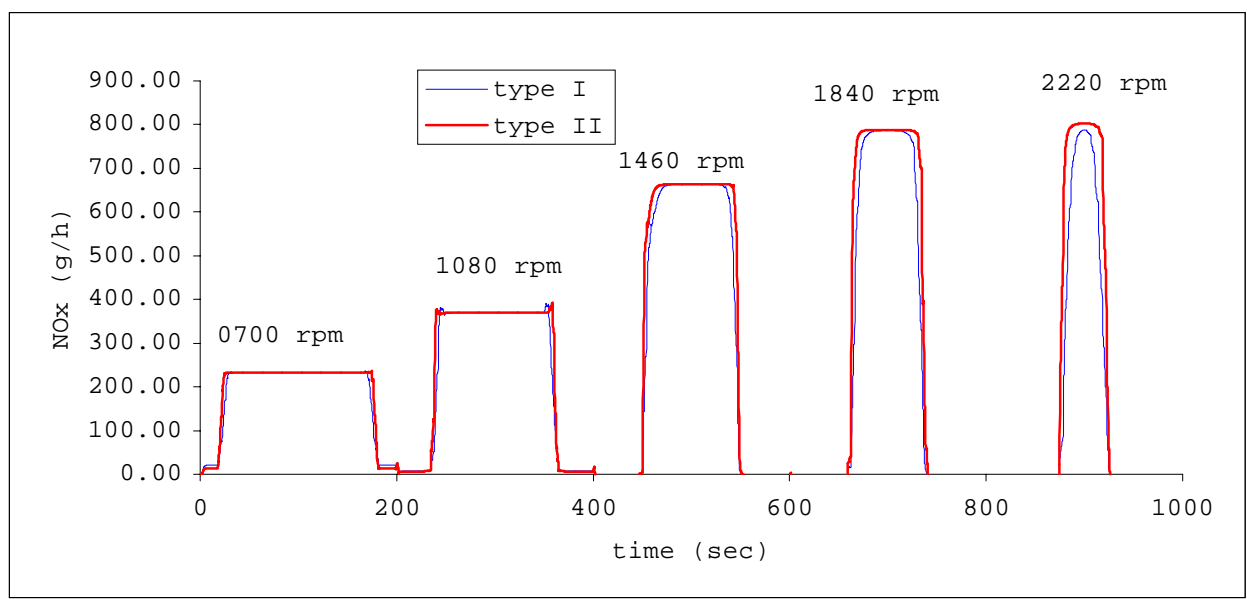

Figure 4.24b Oxides of nitrogen emissions for the two types of proportional governors considered

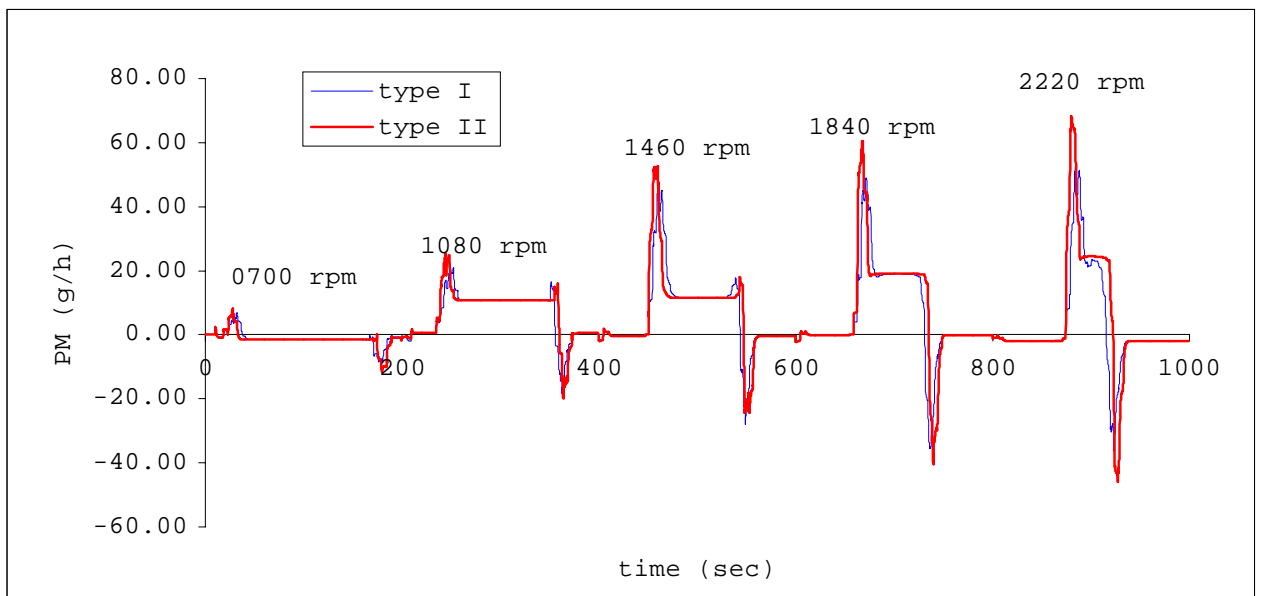

Figure 4.24c Particulate matter emissions for the two types of proportional governors considered 


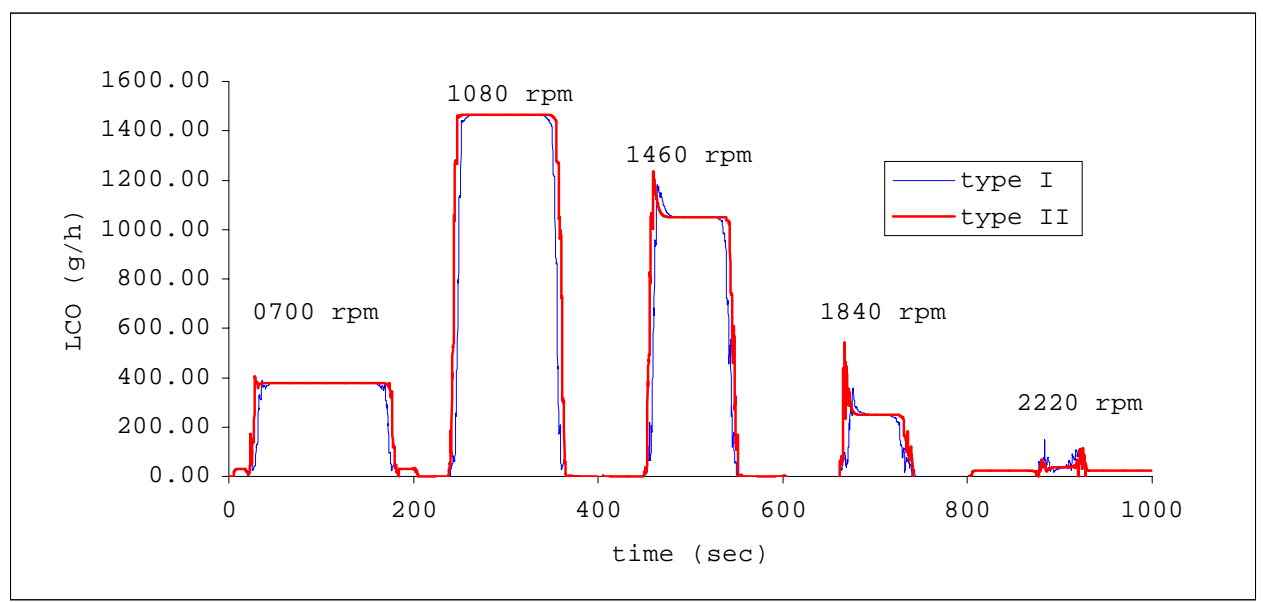

Figure 4.24d Carbon monoxide emissions for the two types of proportional governors considered

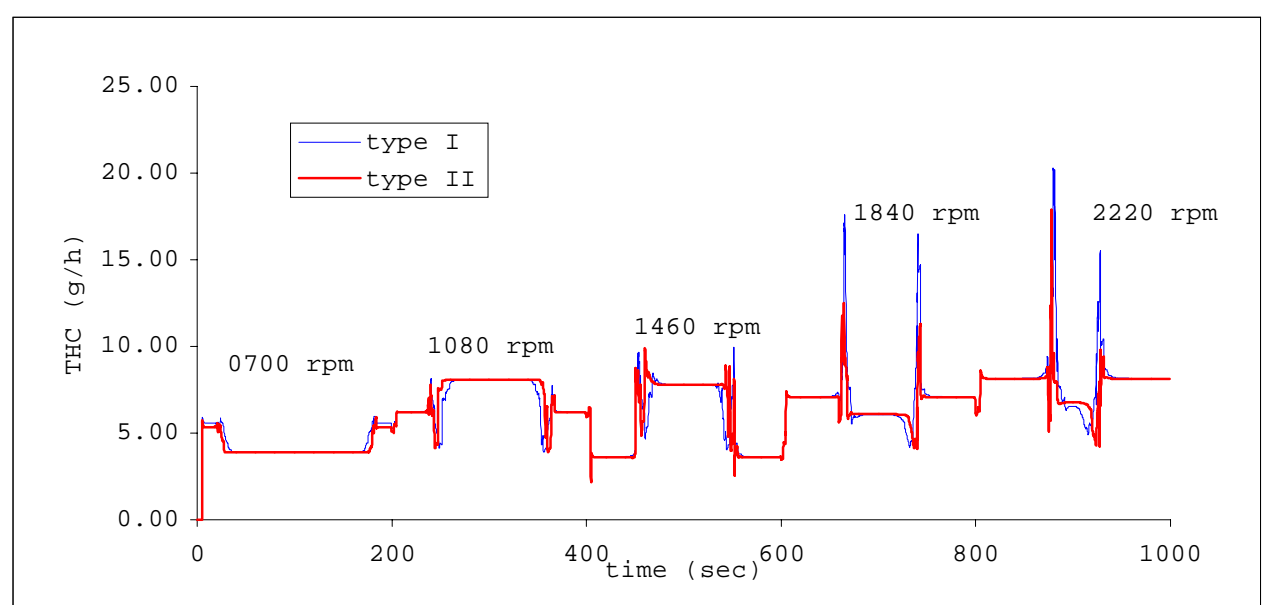

Figure 4.24e Total hydrocarbon emissions for the two types of proportional governors considered

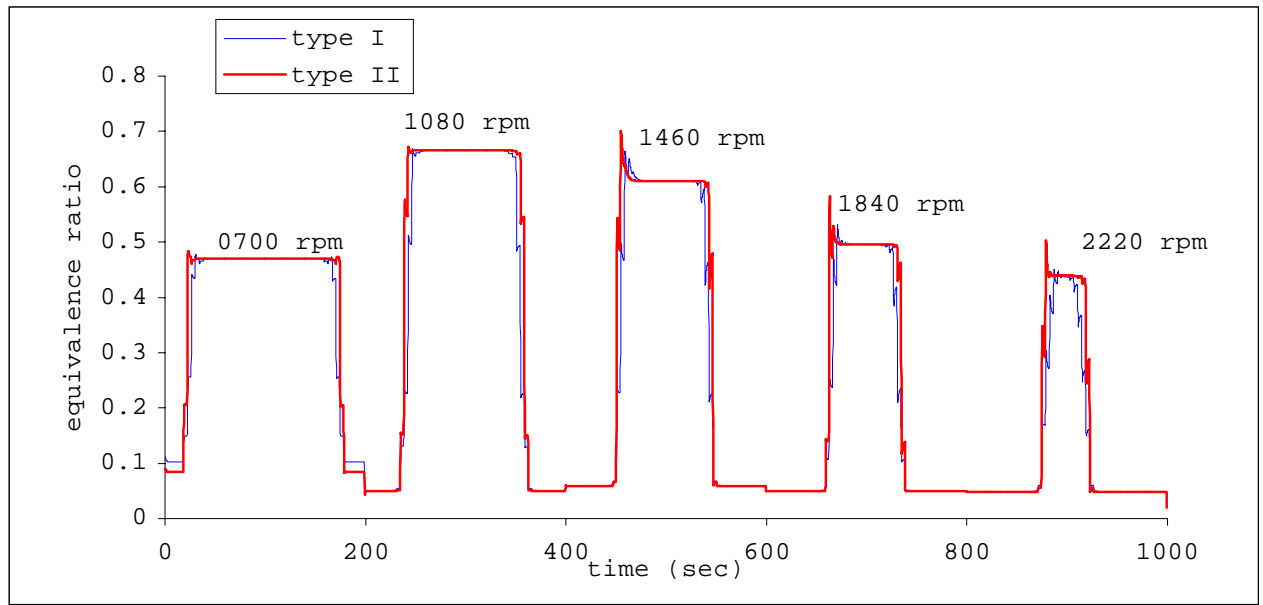

Figure 4.24f Fuel to air equivalence ratio for the two types of proportional governors considered 


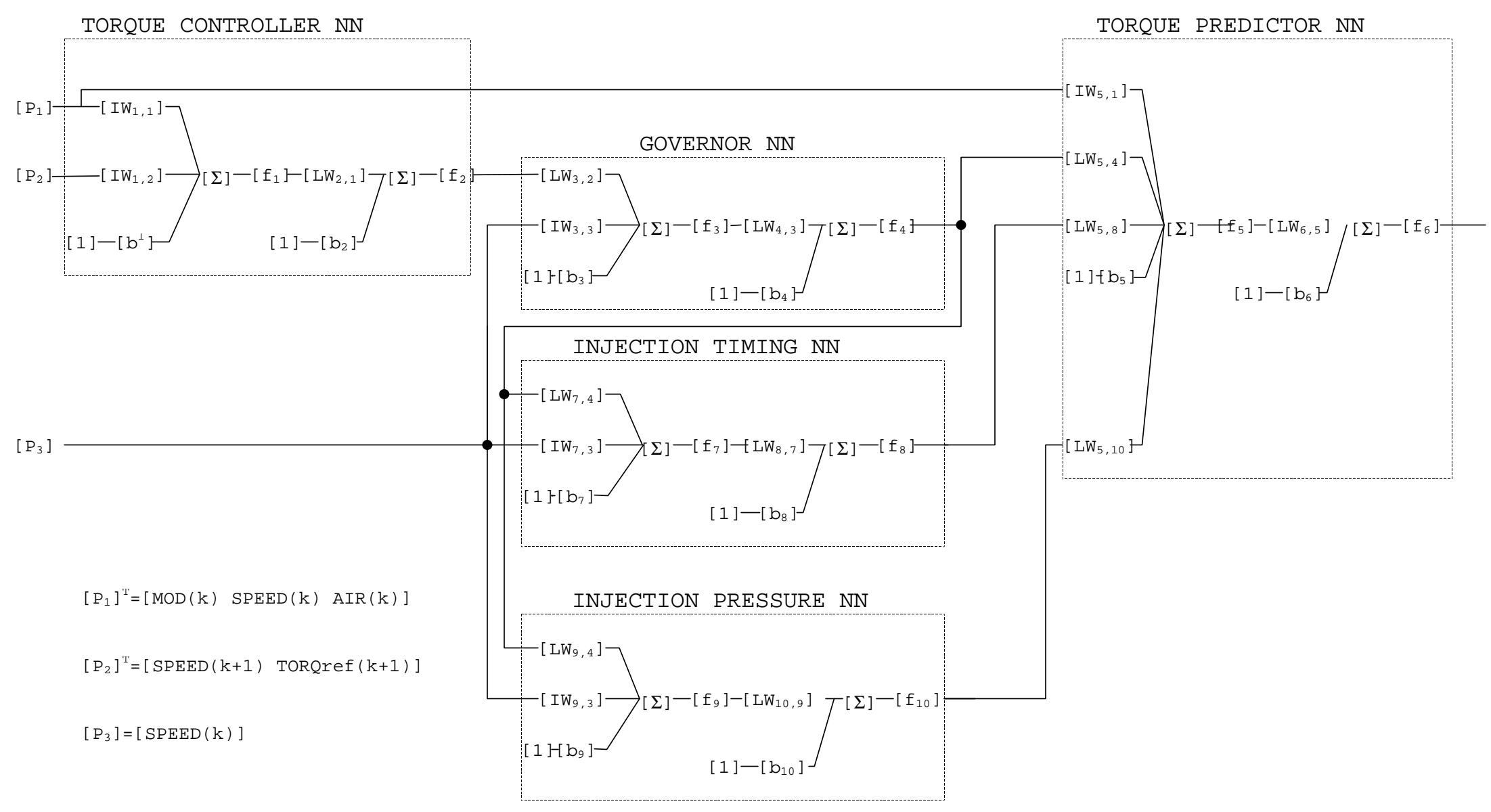

Figure 4.25 Combined neural network layout for governed engine operation 


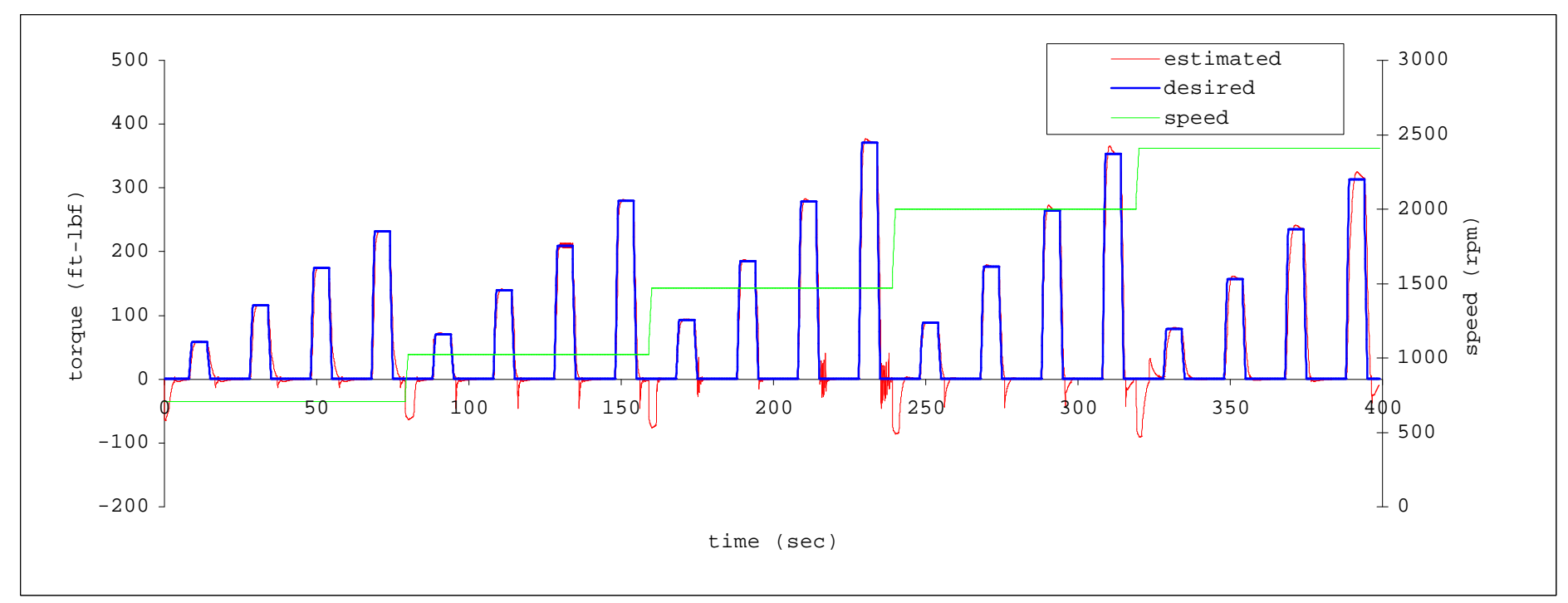

Figure 4.26a Tracking performance over pulsed torque transients with PI control for proportional governor I

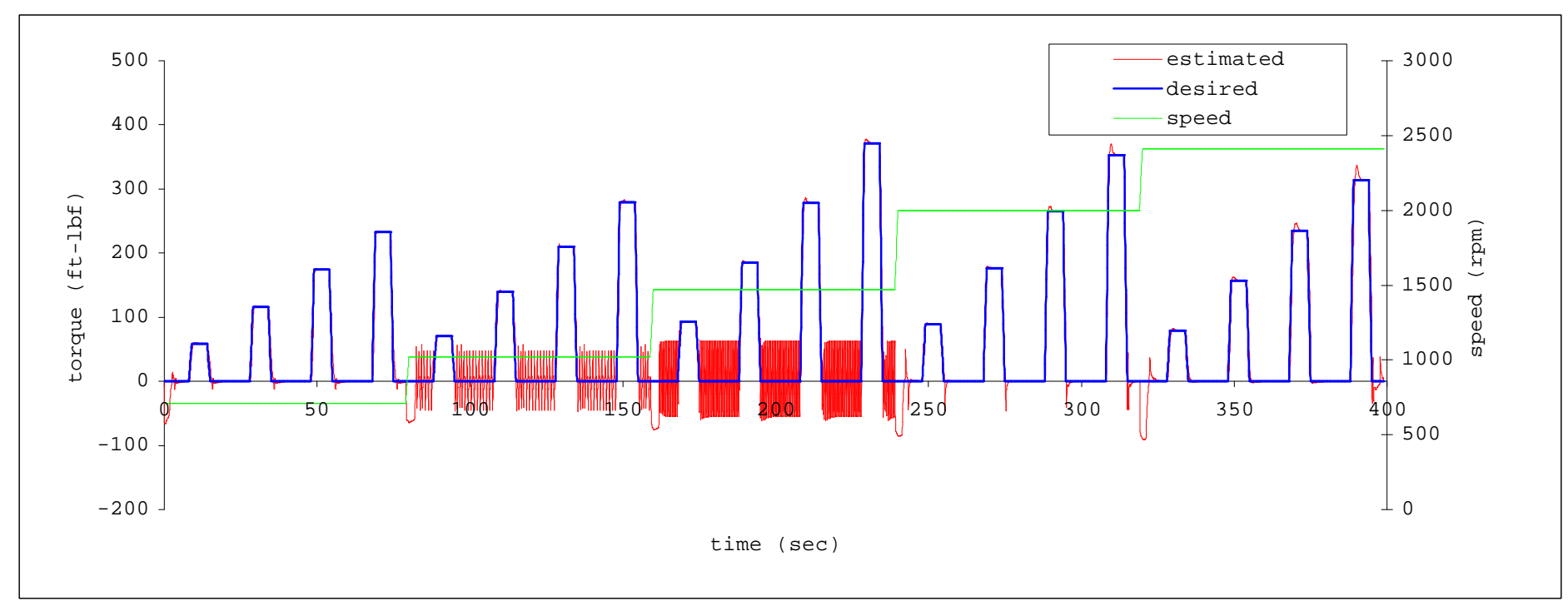

Figure 4.26b Tracking performance over pulsed torque transients with PI control for proportional governor II 


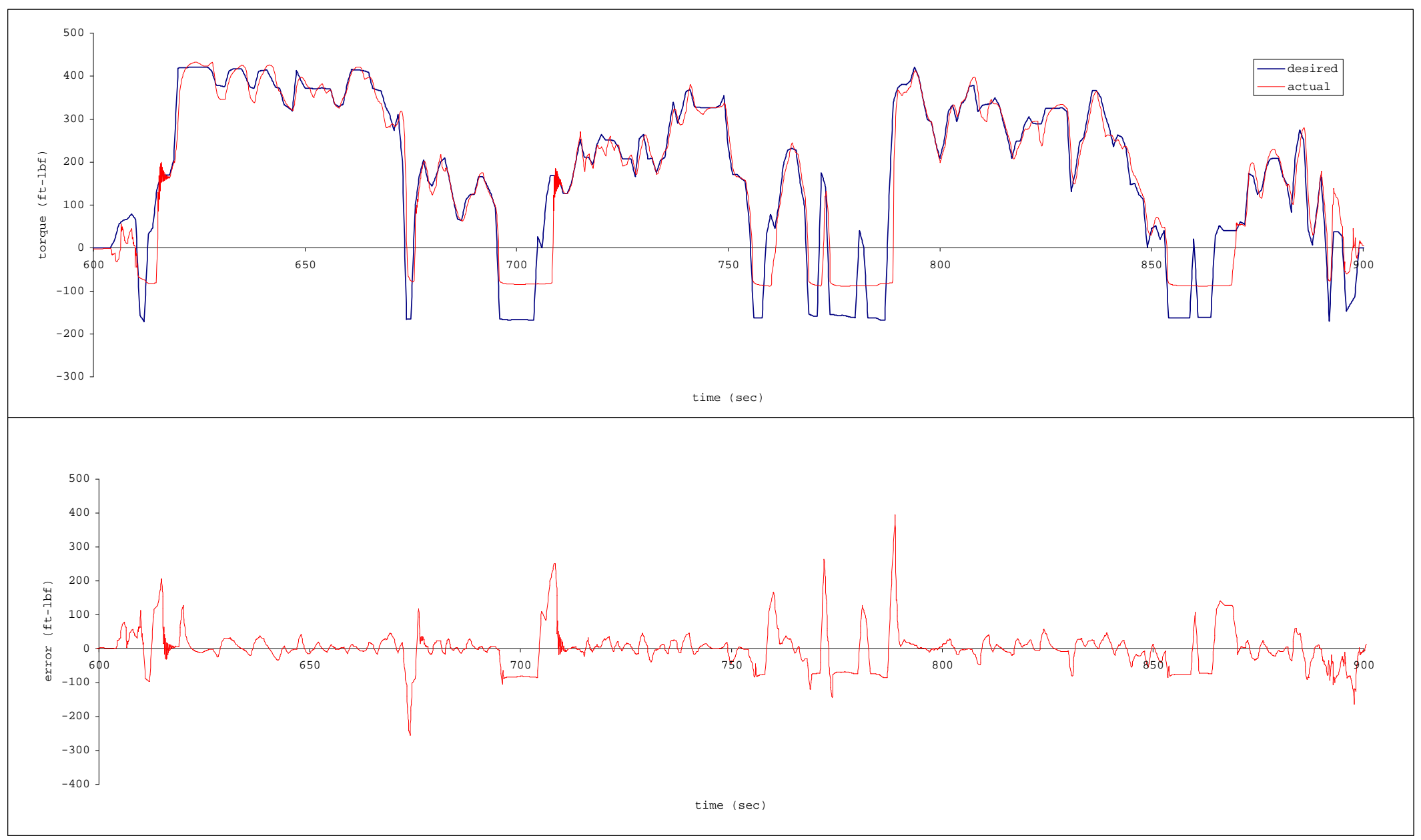

Figure 4.27a Desired and actual torque over the FTP cycle with PI torque control for proportional governor I 


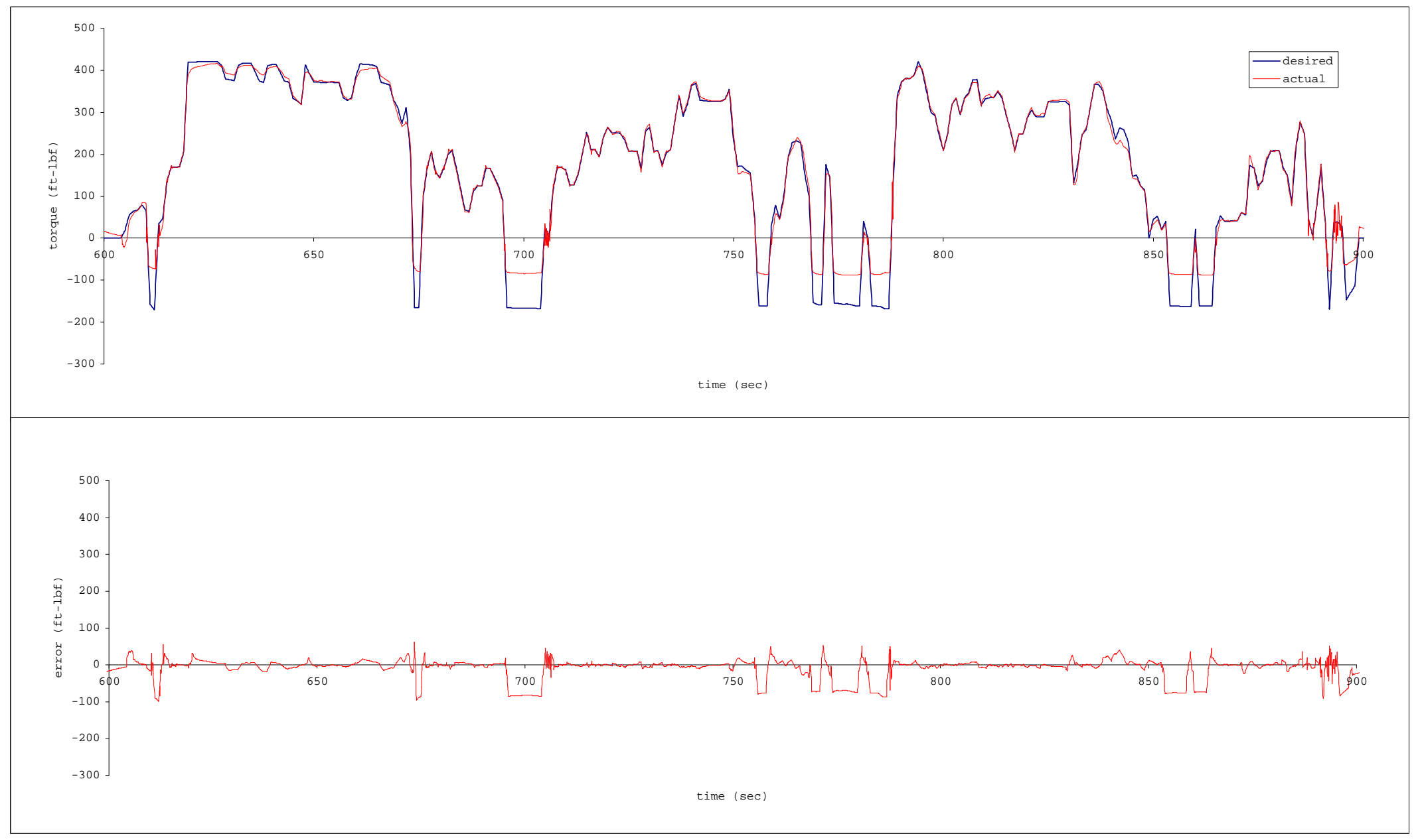

Figure 4.27b Desired and actual torque over the FTP cycle with NN, Ir=0.005, torque control for proportional governor type I 


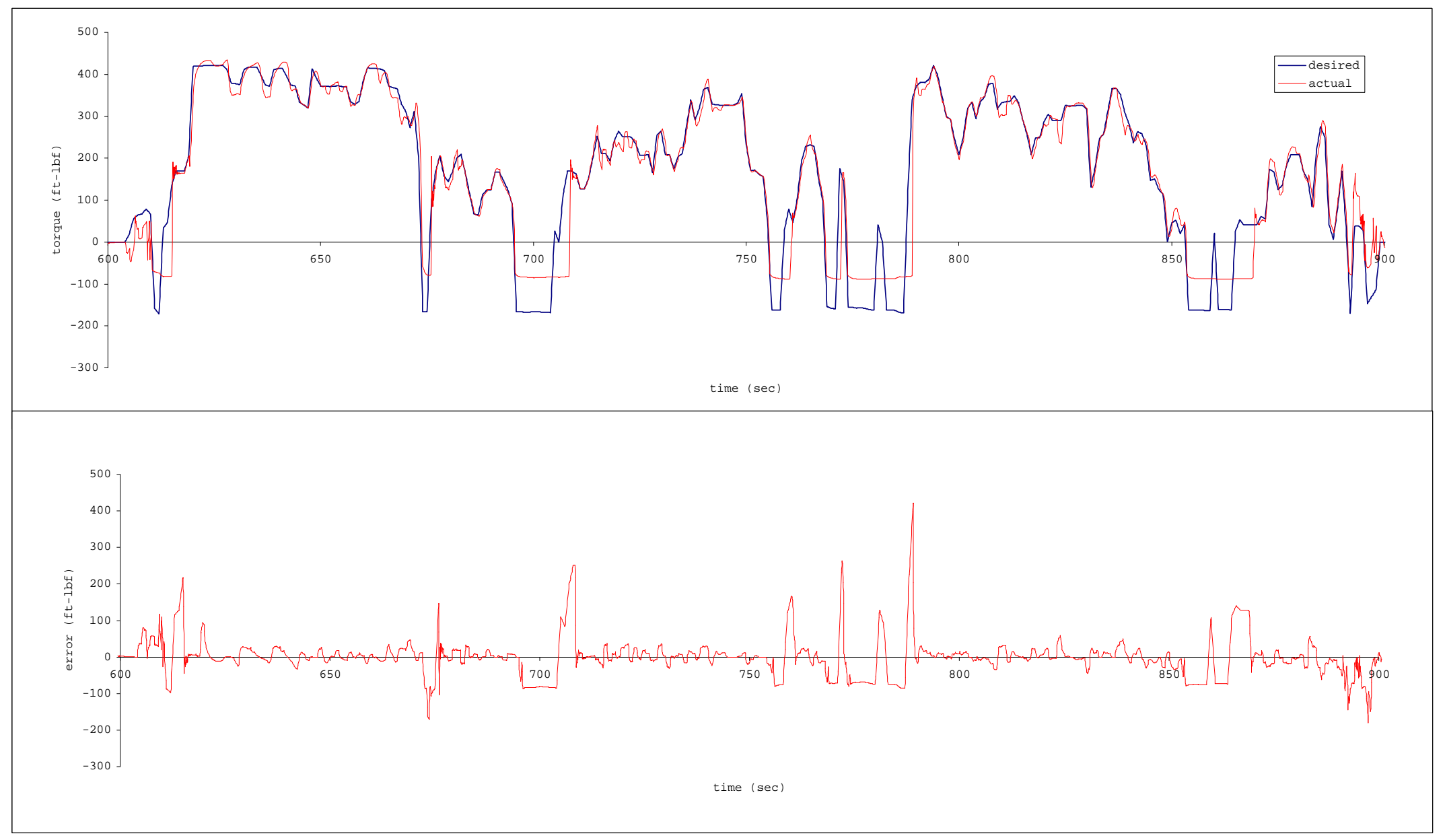

Figure 4.28a Desired and actual torque over the FTP cycle with PI torque control for proportional governor type II 


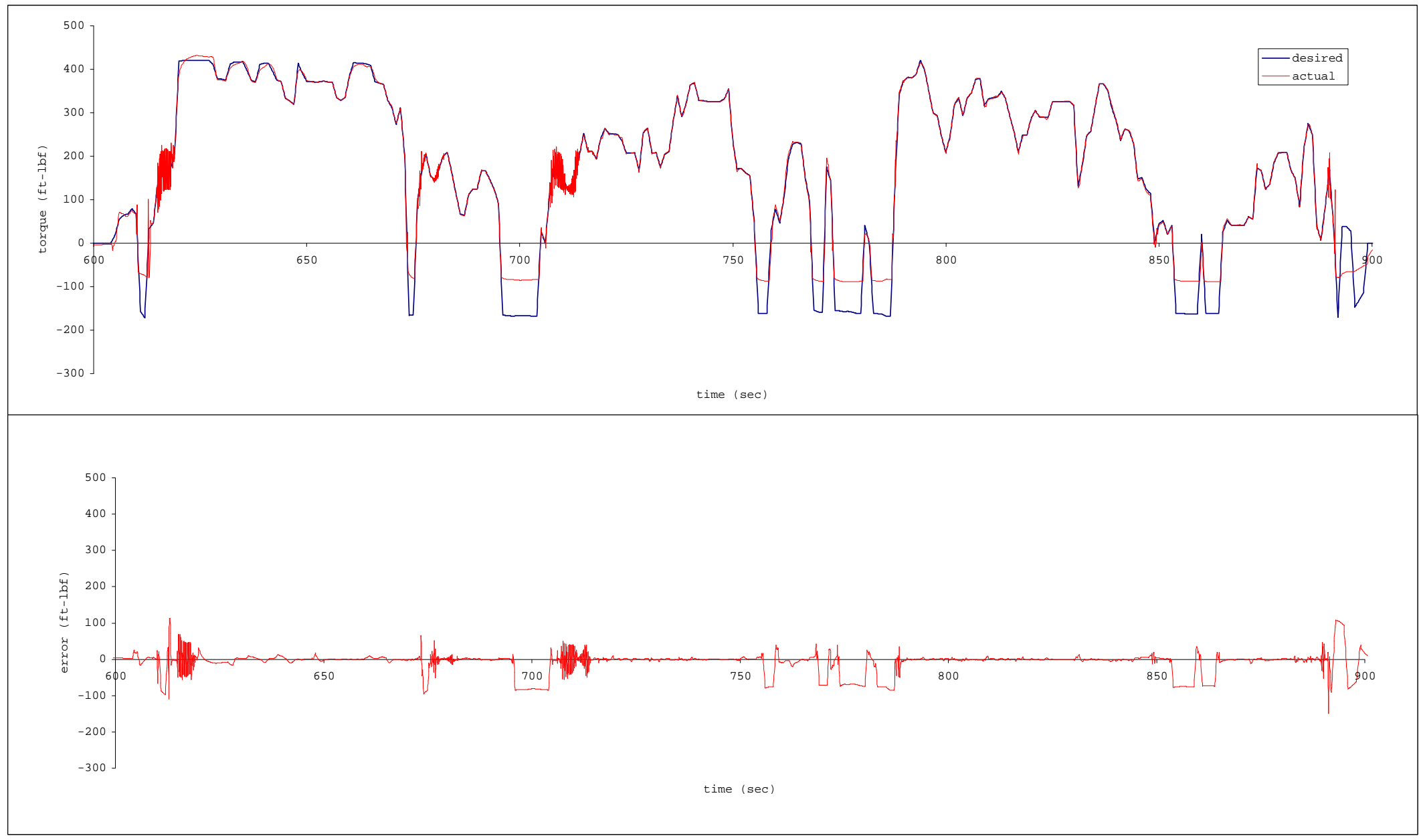

Figure 4.28b Desired and actual torque over the FTP cycle with NN, Ir=0.001, torque control for proportional governor II 


\subsection{Engine Speed Controller Development and Validation}

The closed-loop speed control model is shown in Figure 4.29. The input data to the simulation are the time trajectories for engine load and the accelerator position.

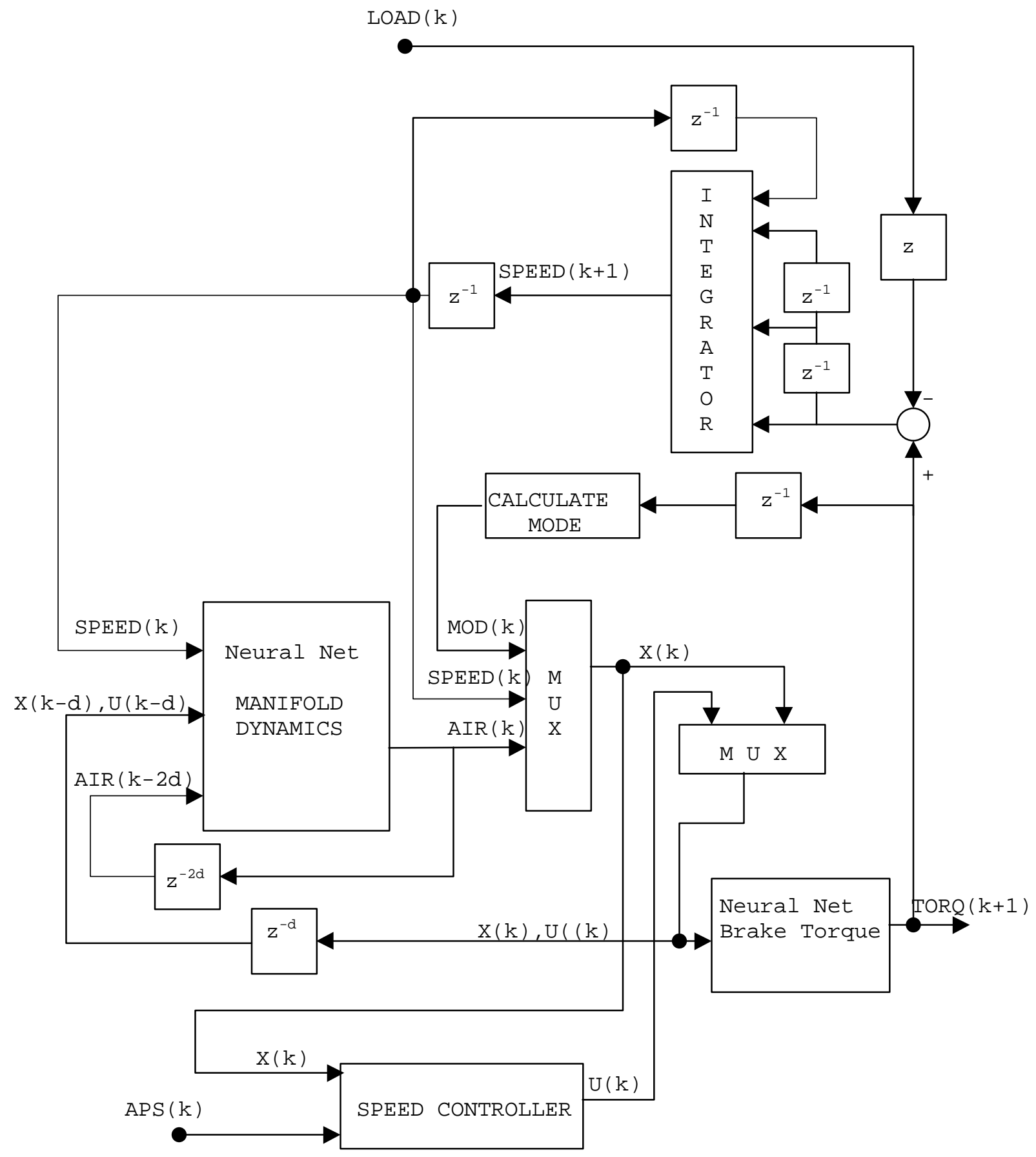

Figure 4.29 Block diagram of the closed-loop speed control 
The speed controller input vector consists of a current engine state and accelerator position. The output control vector consists of the commanded fuel flow rate, injection timing and injection pressure. A three-point fourth order integration scheme was used to calculate the engine speed, as shown in Figure 4.29.

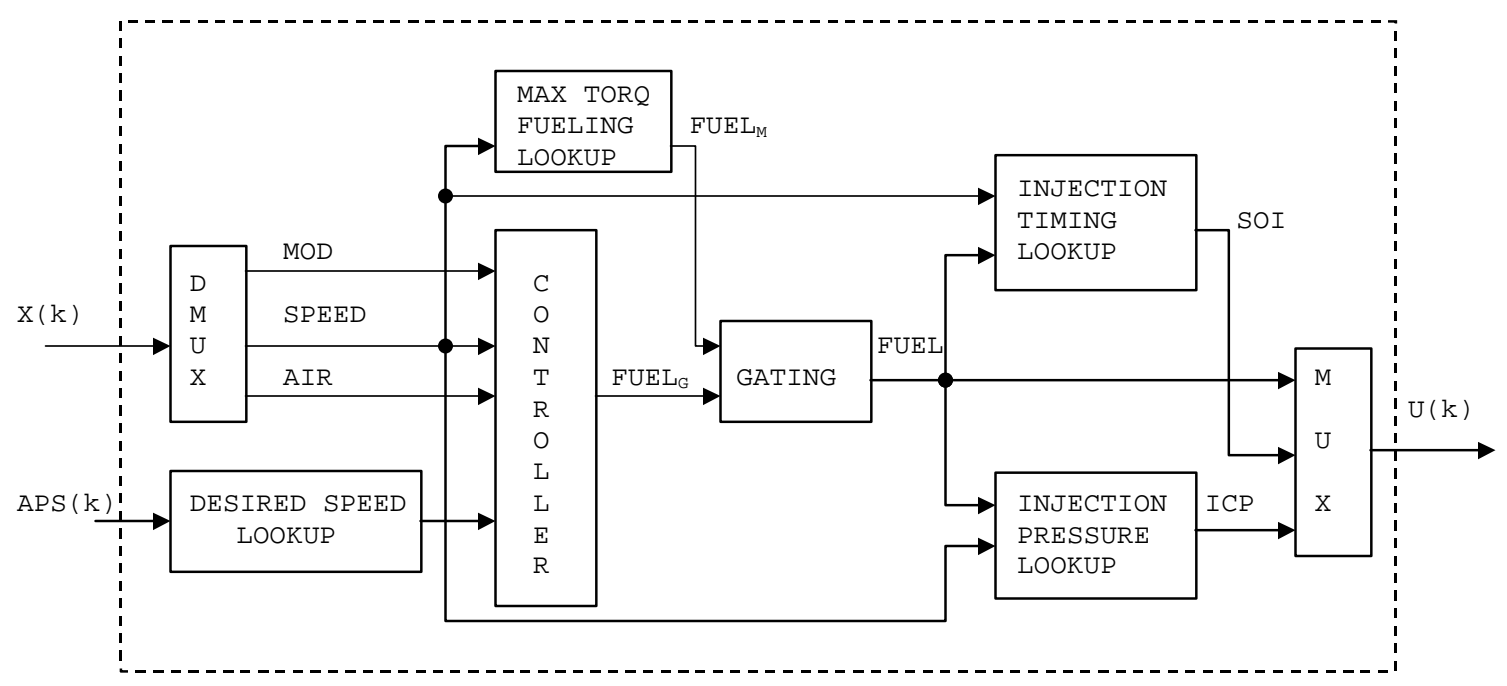

Figure 4.30 Schematic of the engine speed controller

The details of the used speed controller are shown in Figure 4.30. Firstly, the accelerator position signal was used to lookup a desired speed. Secondly, the fuel flow rate was determined using the inverse dynamics controller and compared to the maximum torque fueling, which was looked up as a function of the current engine speed. The gating module was simply used to limit the controller output to the maximum torque fueling. Finally, the injection timing and pressure were looked up using engine speed and the commanded fuel flow rate.

The reduction program was used to generate the look-up data for the desired engine speed and maximum torque limited fueling. Though not necessary for the current implementation, the generated lookup tables were modeled using two NNs in order to retain the ease of accommodating any extra dependencies in a future design. The reconstructed desired speed and maximum torque limited fueling are shown in Figures 4.31a and 4.31b, respectively. The gating 
module was modeled using a single hidden NN, which was trained to calculate the minimum of two signals in the range from -1.0 to 1.0. Also, a single linear neuron (perceptron) with four input nodes was used to calculate the engine speed where the input weights were assigned appropriate values to emulate the three-point integration scheme. This approach was necessary in order to allow for back-propagating the speed tracking error when adapting the controller online. The NN architectures used are shown in Table 4.10.

The combined neural network used in simulating closed-loop speed control is shown in Figure 4.32. The error between predicted and desired engine speed was back-propagated through the speed and torque predictors NNs, the injection timing and pressure NNs, the gating NN, and the inverse dynamics NN. The integral network consisted of fifteen non-fully connected layers and was externally connected to the manifold dynamics $\mathrm{NN}$ and emissions prediction NNs. The speed controller weights were initialized to perform as a proportional controller and the standard back-propagation algorithm was used to adapt the weights of the controller NN while the other NNs weights were frozen at the values acquired through offline training.

Table 4.10 Speed controller neural network architectures

\begin{tabular}{|l|r|r|r|}
\hline \multirow{2}{*}{} & \multicolumn{2}{|c|}{ Number of Nodes } \\
\cline { 2 - 4 } & Input & Hidden & Output \\
\hline Desired speed & 1 & 2 & 1 \\
\hline Maximum torque limited fueling & 1 & 4 & 1 \\
\hline Minimum gating & 2 & 16 & 1 \\
\hline Inverse dynamics & 4 & 4 & 1 \\
\hline
\end{tabular}


The simulation was conducted for a stepped signal of the accelerator, from $0 \%$ to $100 \%$ at increments of $5 \%$, and the engine load was set to a constant percentile value $(0 \%, 20 \%, 40 \%$, $60 \%$, or $80 \%$ ) of the maximum torque at the desired engine speed, as shown in Figure 4.33a. For comparison purposes, the simulation was also conducted for a PI speed controller with heuristically determined gains. Excellent speed tracking was predicted for the PI control and the $\mathrm{NN}$ controller which was adapted at a learning rate value of $\lambda=0.1$, as shown in Figure 4.33c. The fuel rate was limited by the maximum torque value under high load operation, as shown in Figure $4.33 \mathrm{~d}$. The applied load and the estimated engine torque for both controllers are shown in Figure 4.33e.

In order to estimate the $\mathrm{NN}$ speed controller performance and emissions characteristics, the stepped accelerator test cycle, shown in Figure $4.22 \mathrm{~b}$, was simulated and the weights of the controller NN were frozen at their trained values. The fueling rate characteristics of the NN governor and proportional governor type II are shown in Figure 4.34a. The tight speed tracking of the NN controller resulted in a more aggressive fueling strategy compared to that of type II governor. In turn, the rise in torque was steeper as shown in Figure 4.34b, and over-fueling was higher, as shown in Figure 4.34c. The predicted emissions are shown in Figure 4.35. The NN controller did not result in any apparent increase in $\mathrm{CO}_{2}$ and $\mathrm{NOx}$ emissions. The spikes in PM, $\mathrm{CO}$, and THC emissions were higher for the NN governor compared to the less aggressive type II governor.

The speed tracking was examined for the case when sharp load was applied suddenly at constant position of the accelerator, as shown in Figure 4.36a. For a constant APS value, the applied load spanned the range from $0 \%$ to $80 \%$ at $20 \%$ increments of the maximum torque for the desired engine speed. The simulation was conducted for the NN controller as well as the PI 
controller. For the NN controller, the simulation was conducted for the case the NN weights were frozen at their trained values as well as the case when the weights were adapted online at a learning rate of $\lambda=0.10$. Excellent speed tracking was predicted for the PI control and the NN controller for both frozen and adapted cases, as shown in Figures 4.36b and 4.36c. 


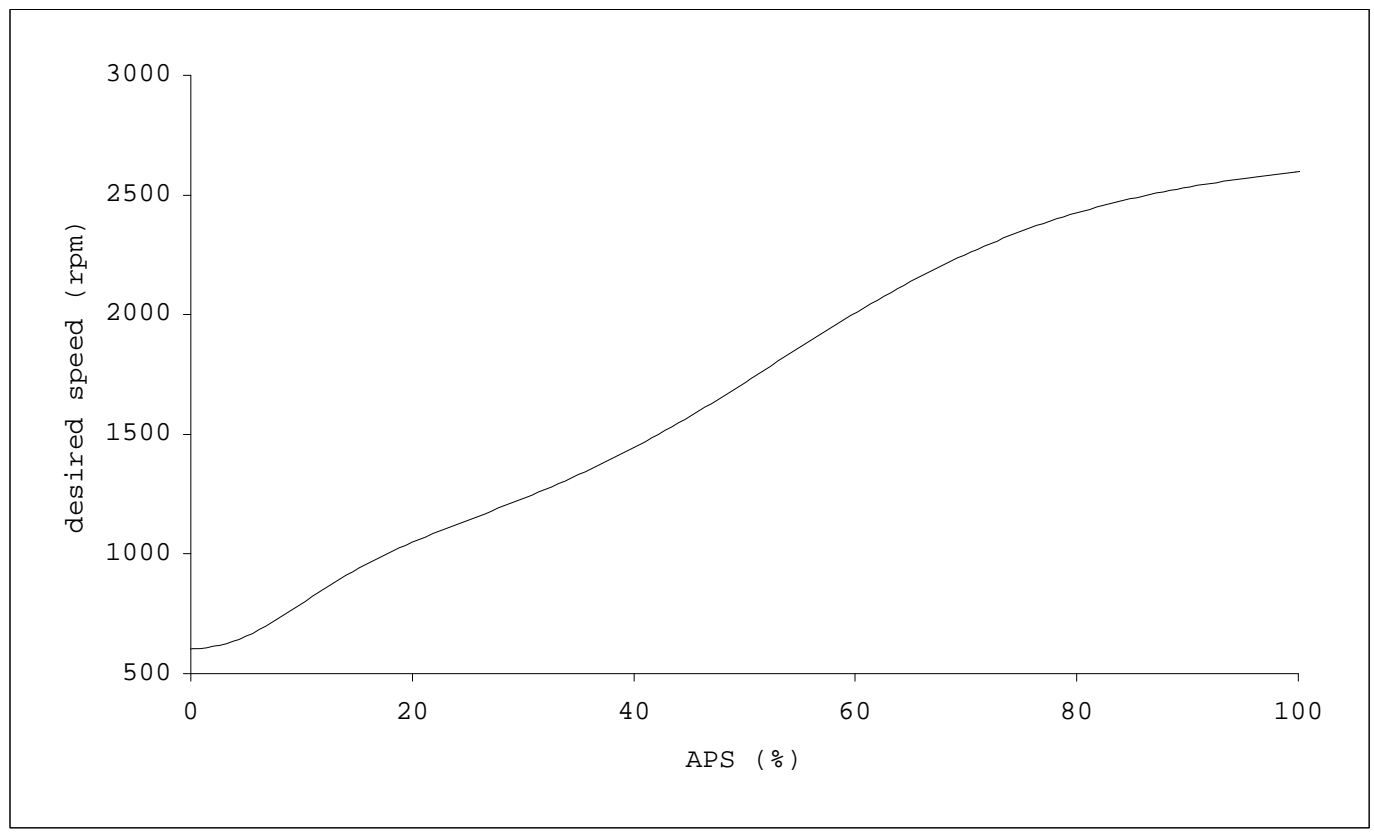

Figure 4.31a Governor desired speed as a function of accelerator position

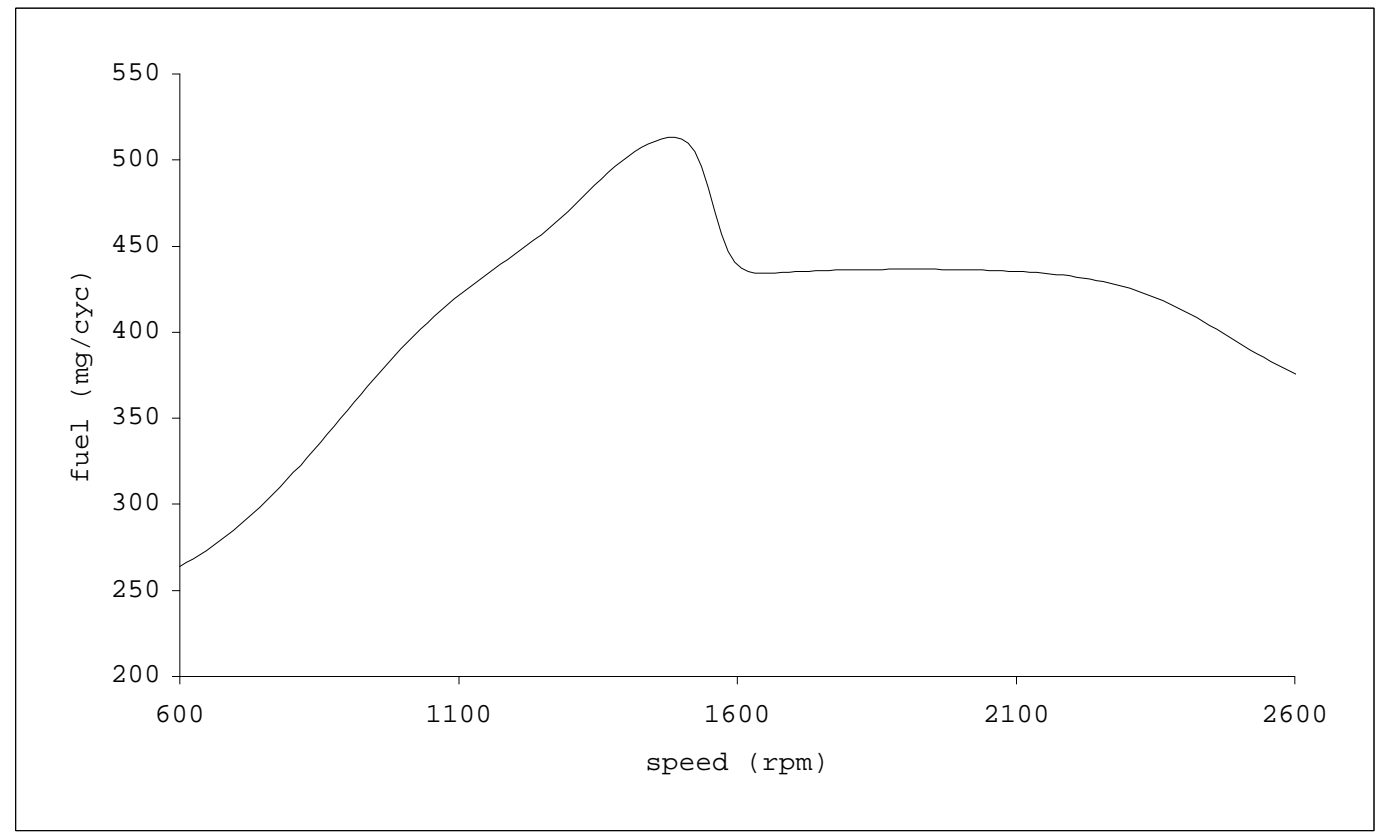

Figure 4.31b Maximum torque fuel flow rate as a function of engine speed 


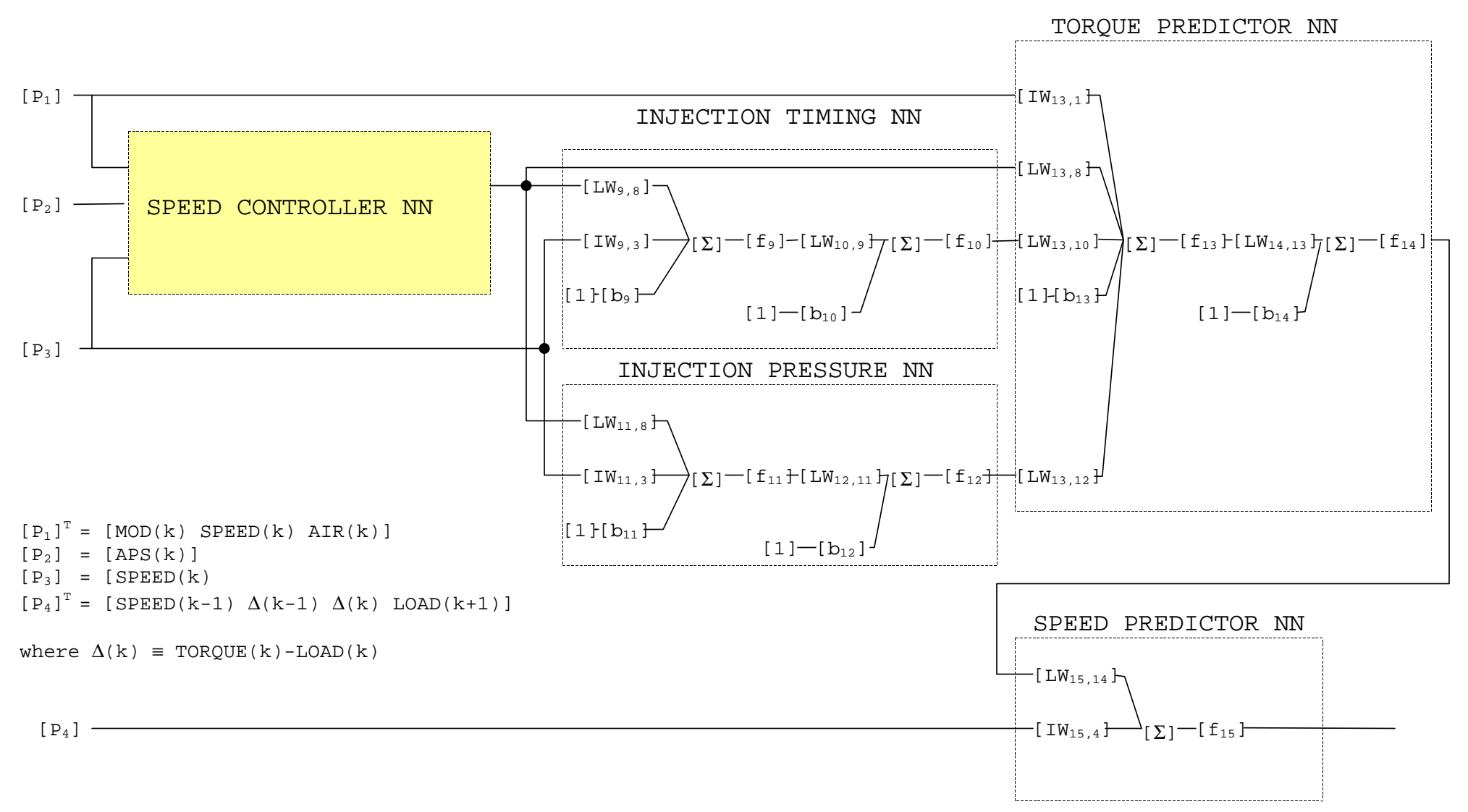

Figure 4.32a Combined neural network layout for closed-loop speed control operation 


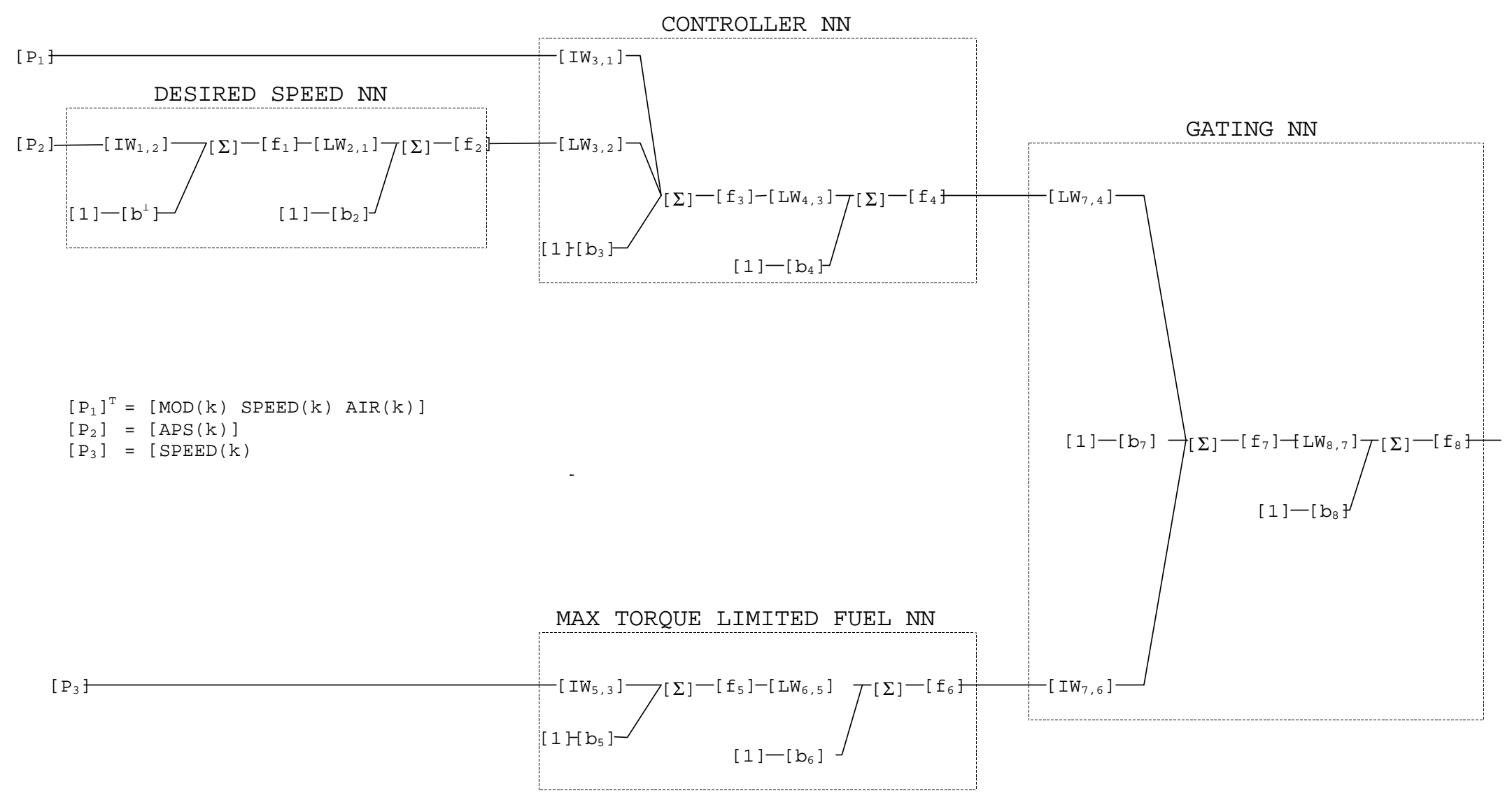

Figure 4.32b Combined neural network layout for engine speed controller 


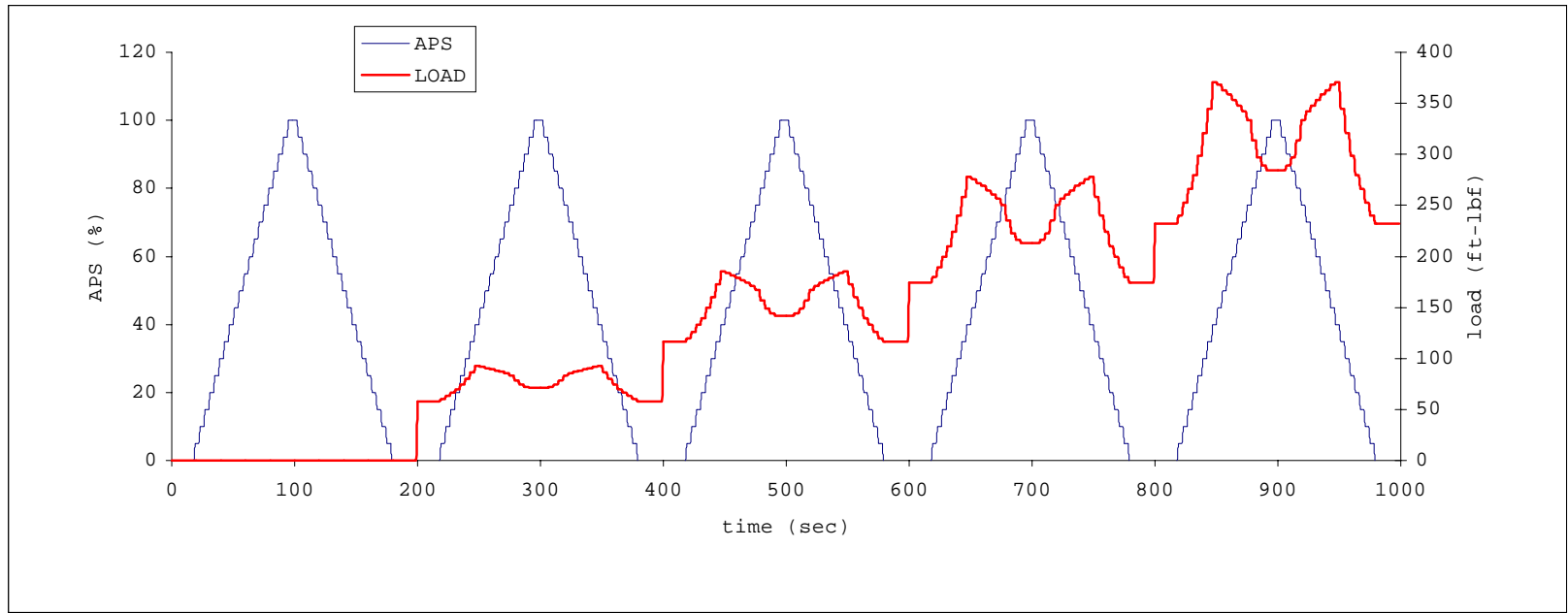

Figure 4.33a Stepped accelerator position signal at different external loads

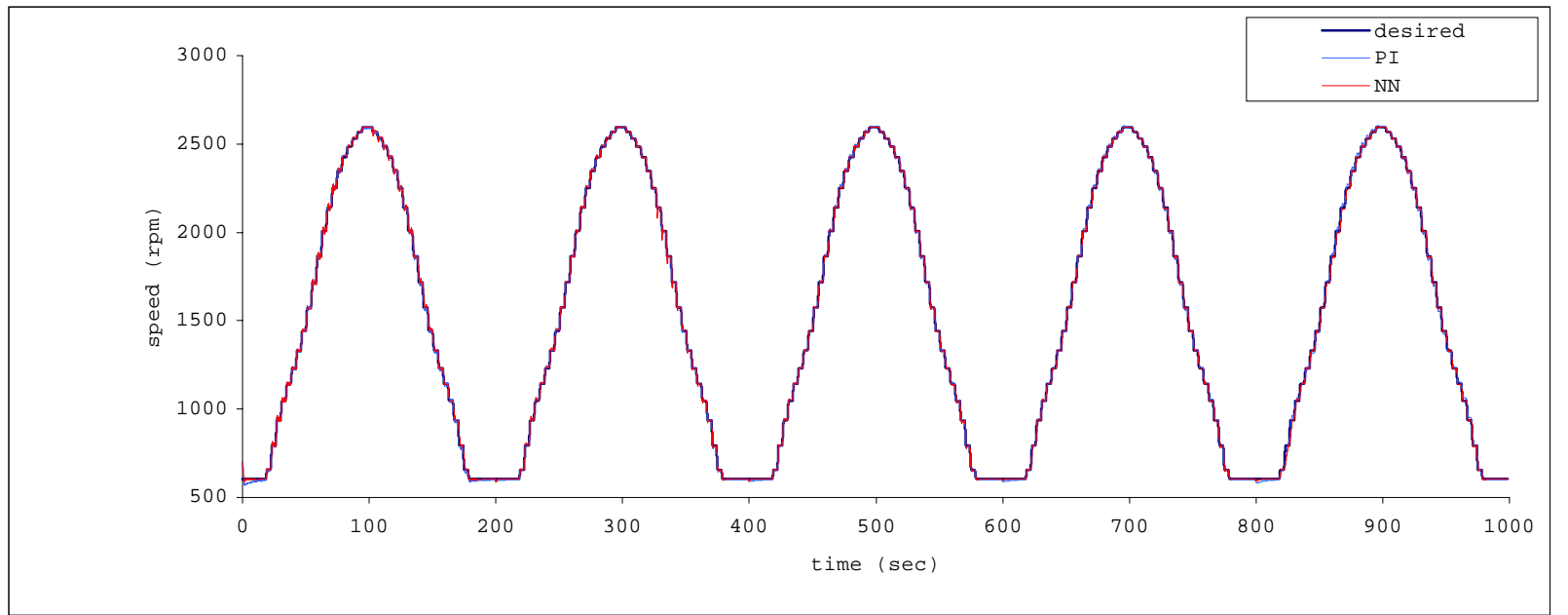

Figure 4.33b Speed tracking performance for the stepped accelerator test cycle

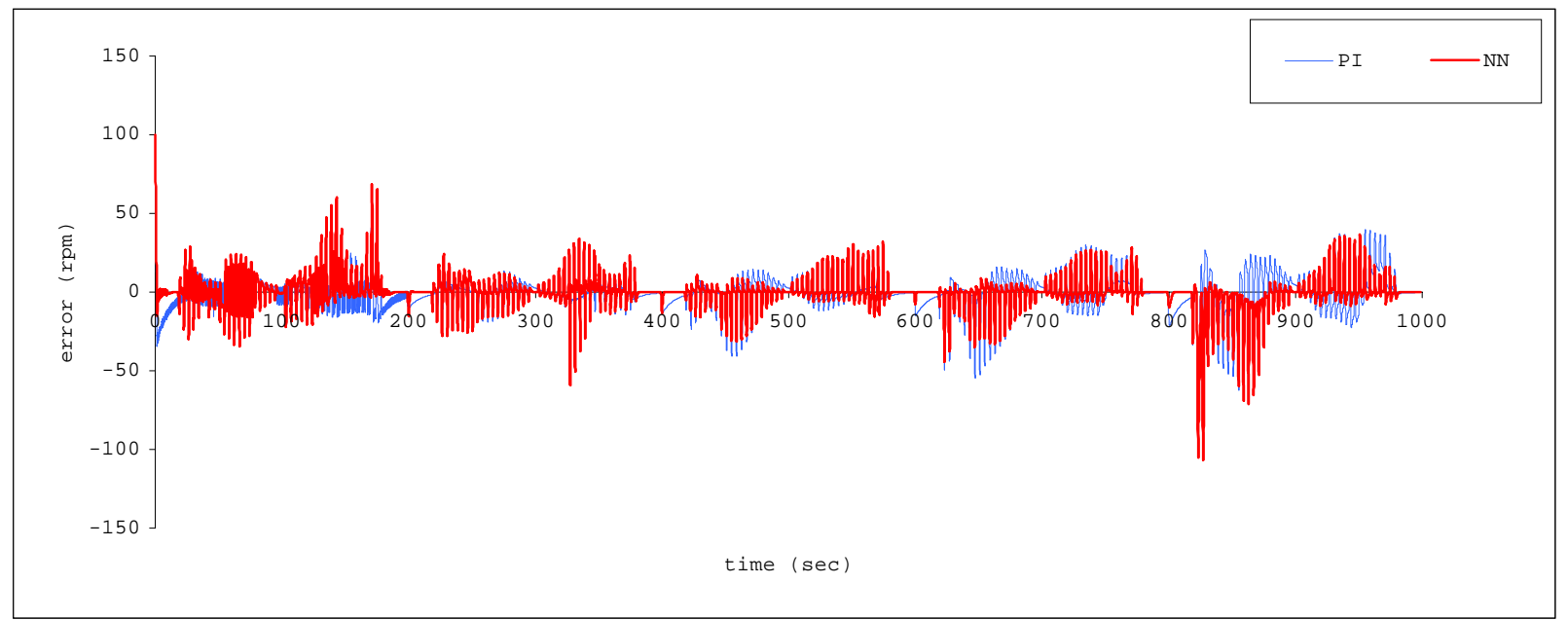

Figure 4.33c Error in speed tracking for the stepped accelerator test cycle 


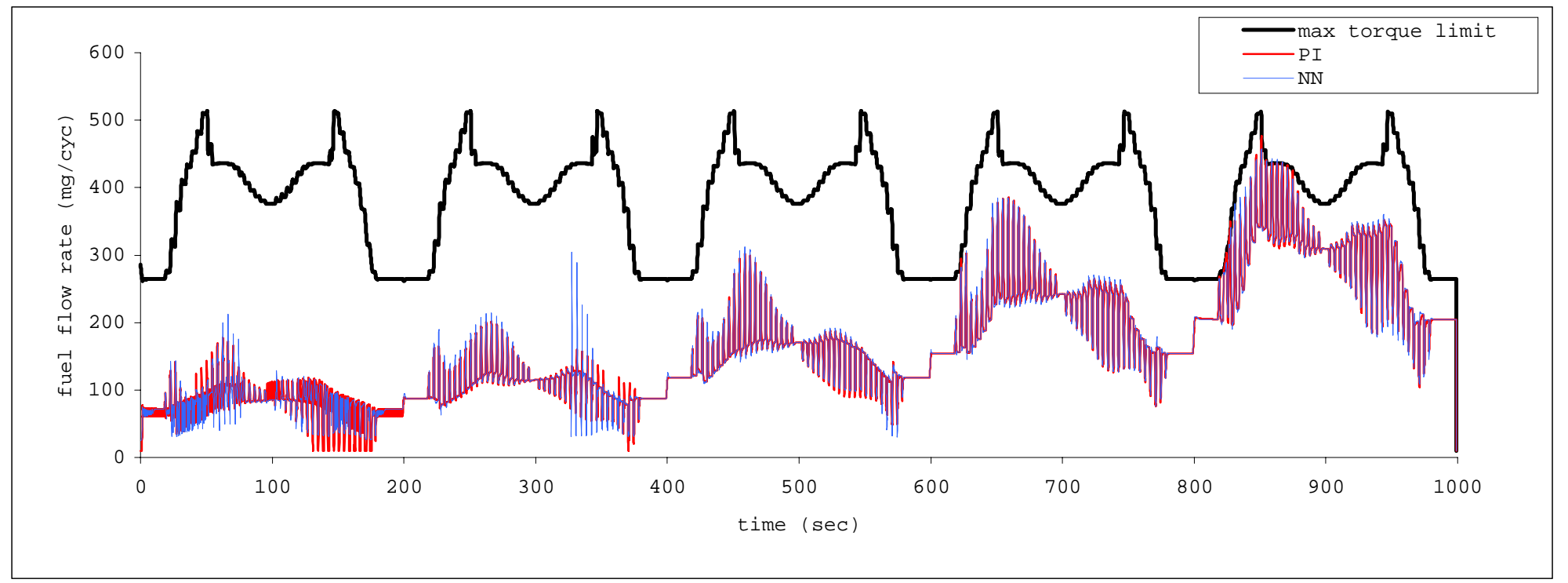

Figure 4.33d Fuel flow rate for closed-loop control of engine speed over the stepped accelerator test cycle

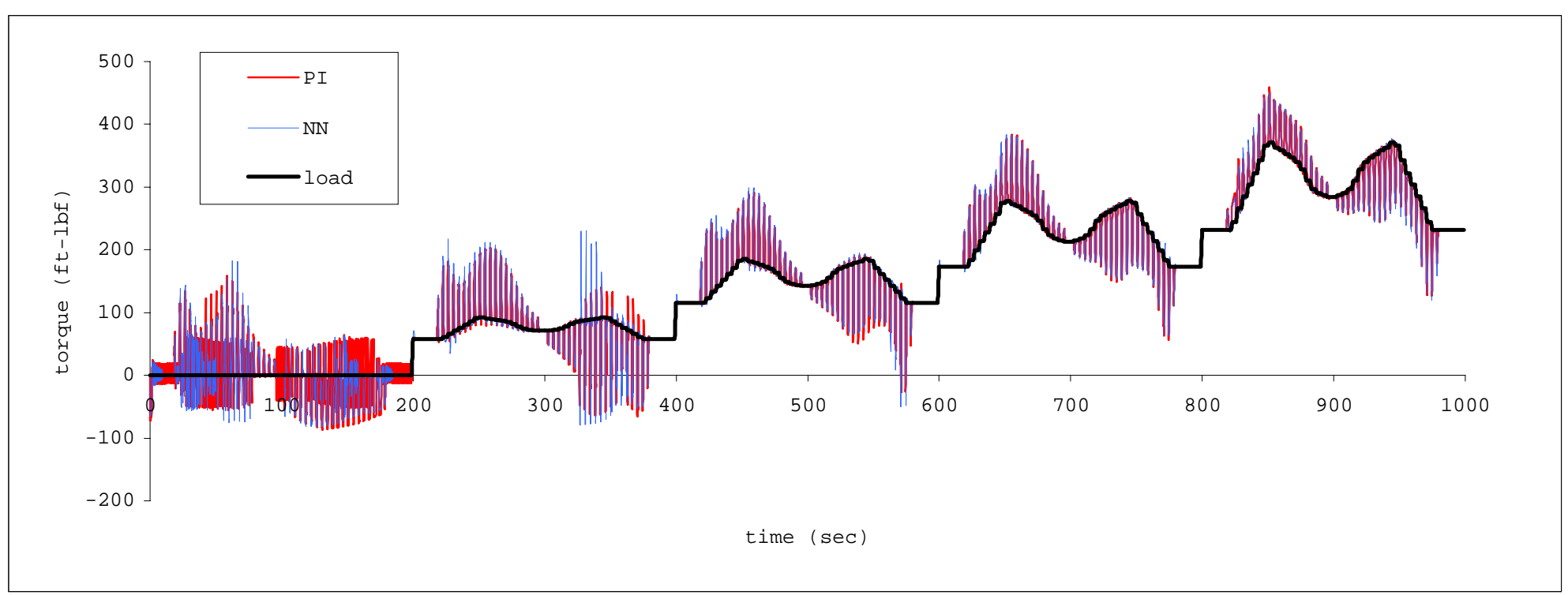

Figure 4.33e Engine torque for closed-loop control of speed over the stepped accelerator test cycle 


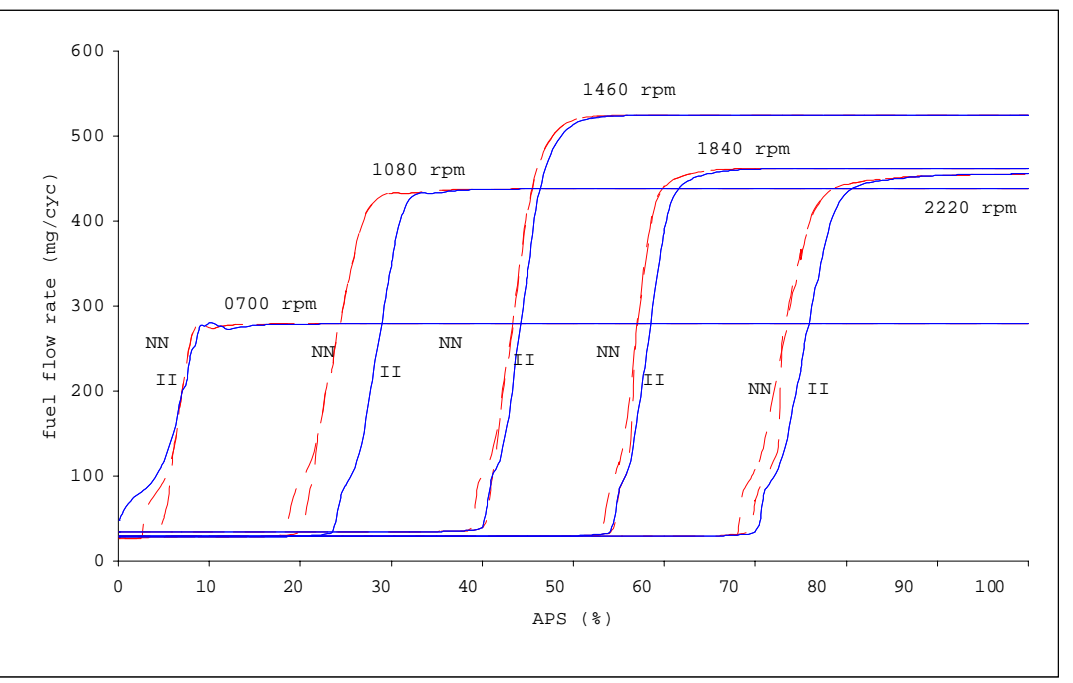

Figure 4.34a Fuel flow rate characteristics for NN-based and type II governors

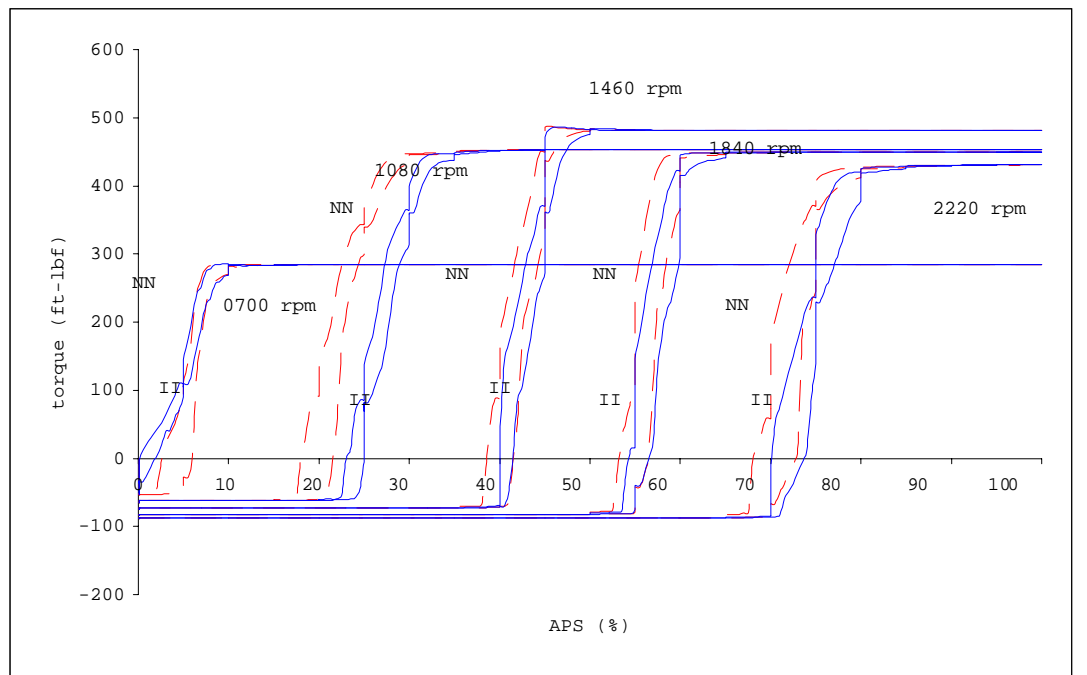

Figure 4.34b Engine torque characteristics for NN-based and type II governors

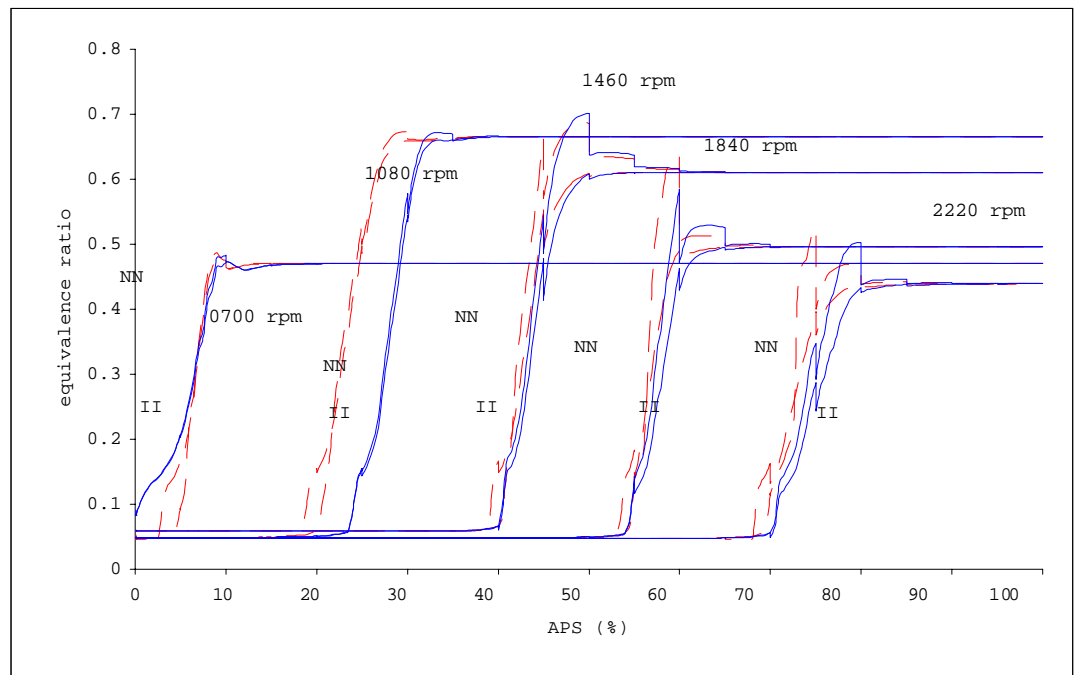

Figure 4.34c Fuel to air ratio characteristics for NN-based and type II governors 


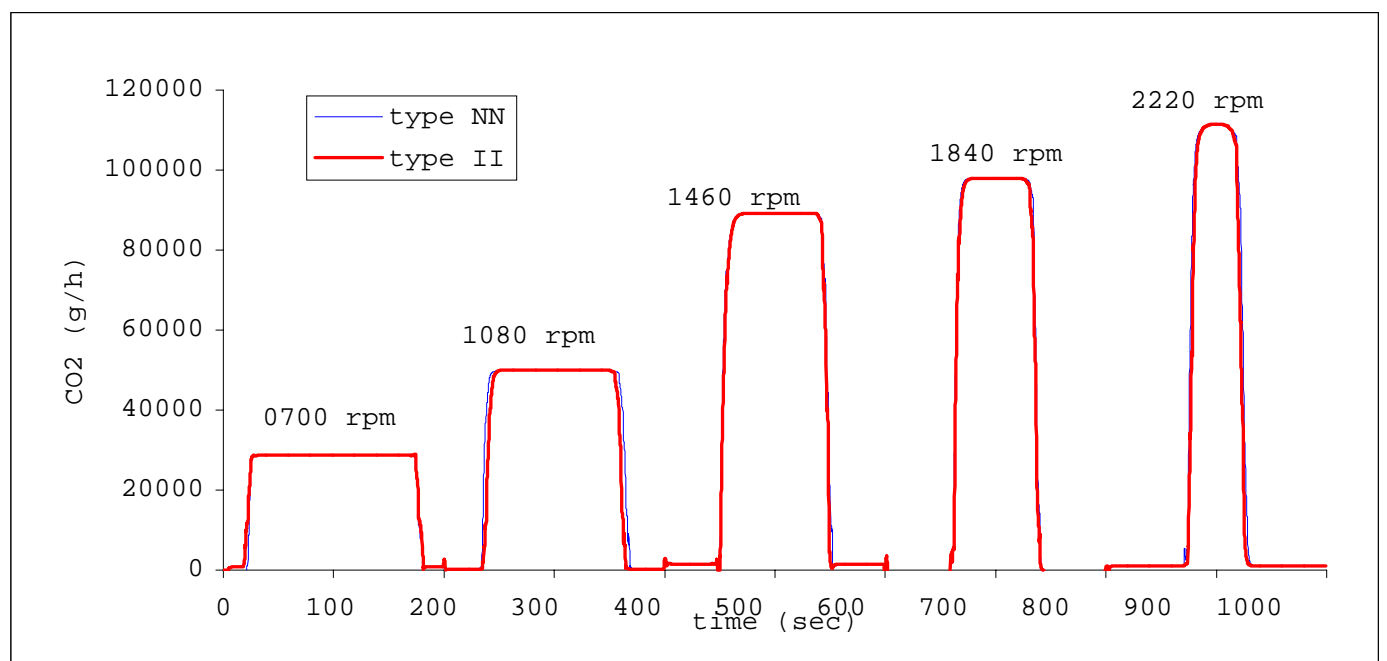

Figure 4.35a Carbon dioxide emissions for NN-based and type II governors

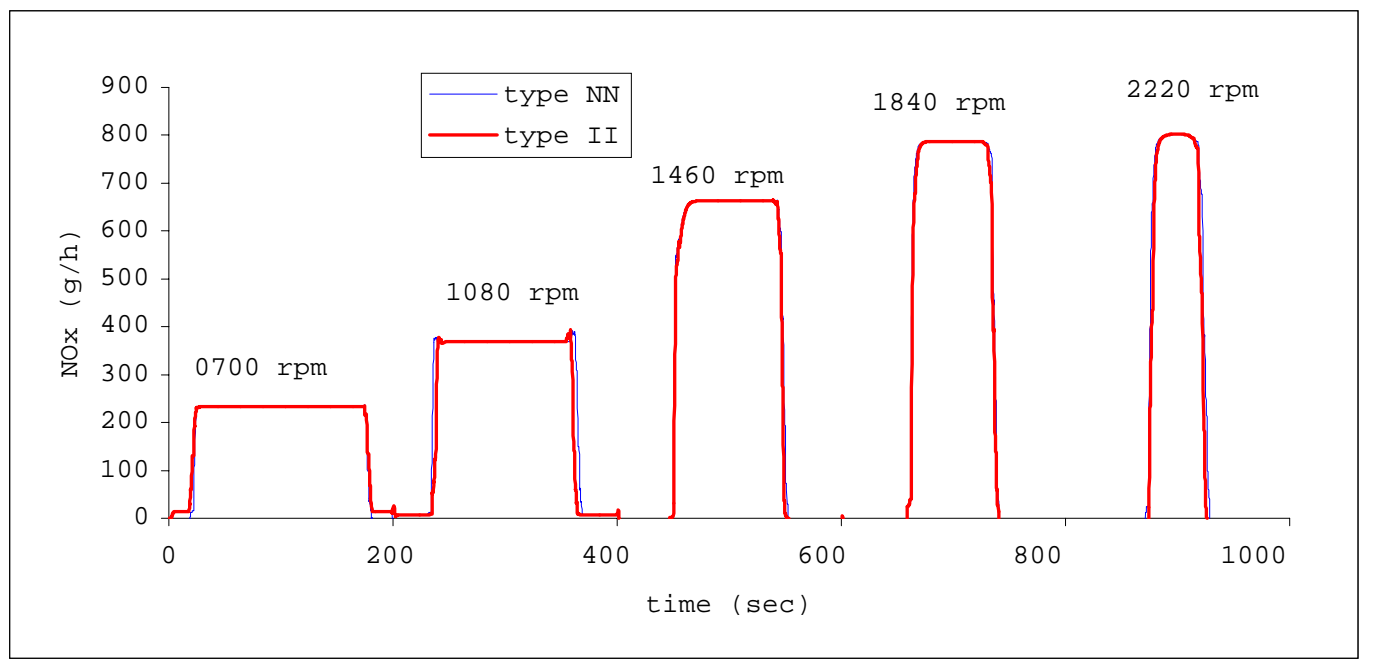

Figure 4.35b Oxides of nitrogen emissions for NN-based and type II governors

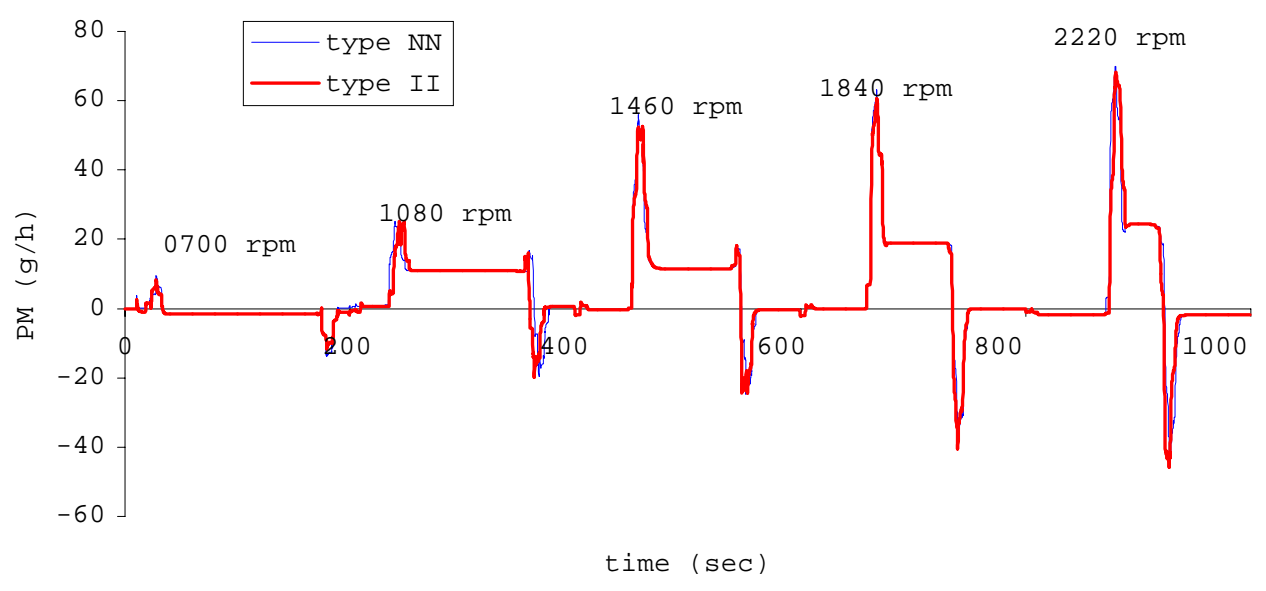

Figure 4.35c Particulate matter emissions for NN-based and type II governors 


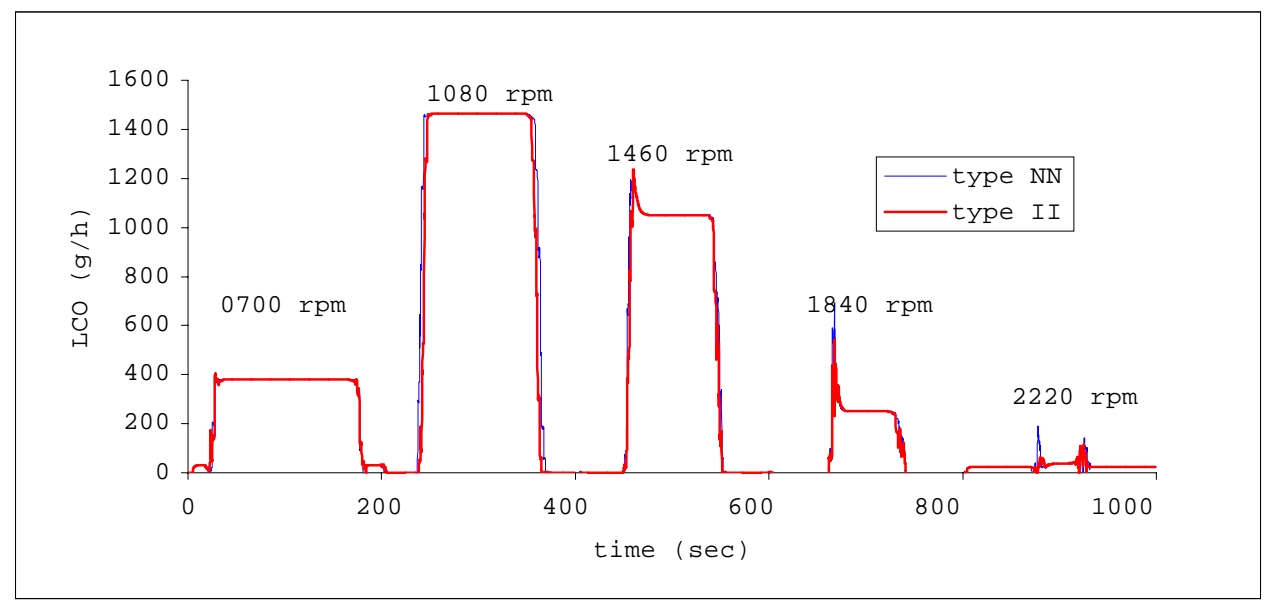

Figure 4.35d Carbon monoxide emissions for NN-based and type II governors

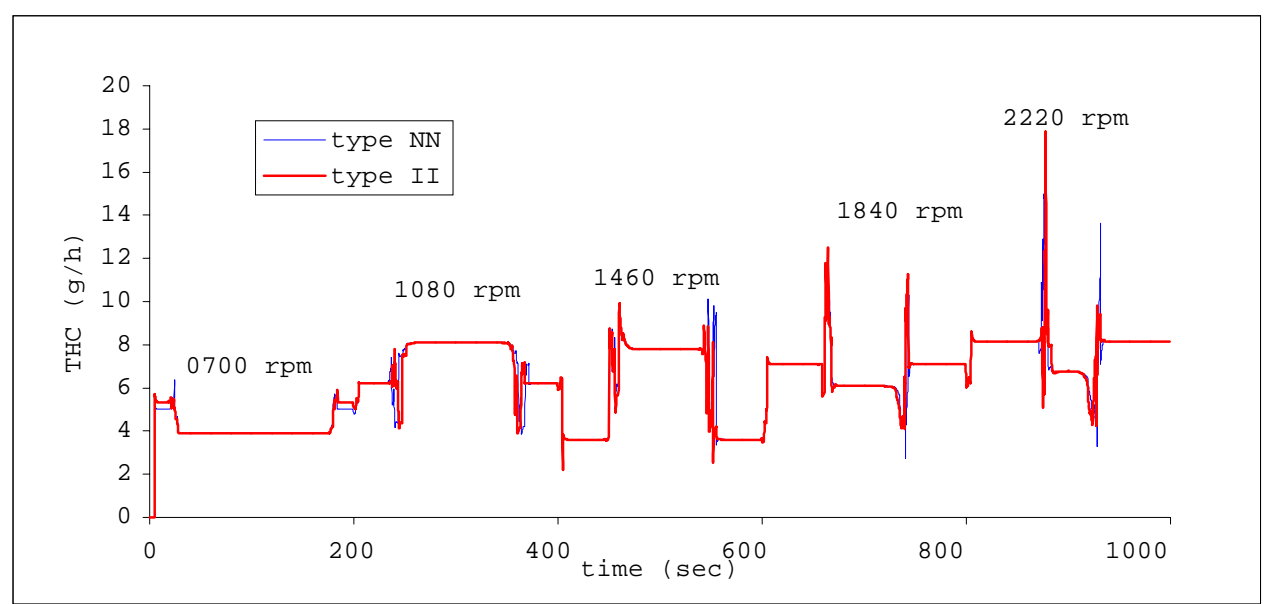

Figure 4.35e Total hydrocarbon emissions for NN-based and type II governors 


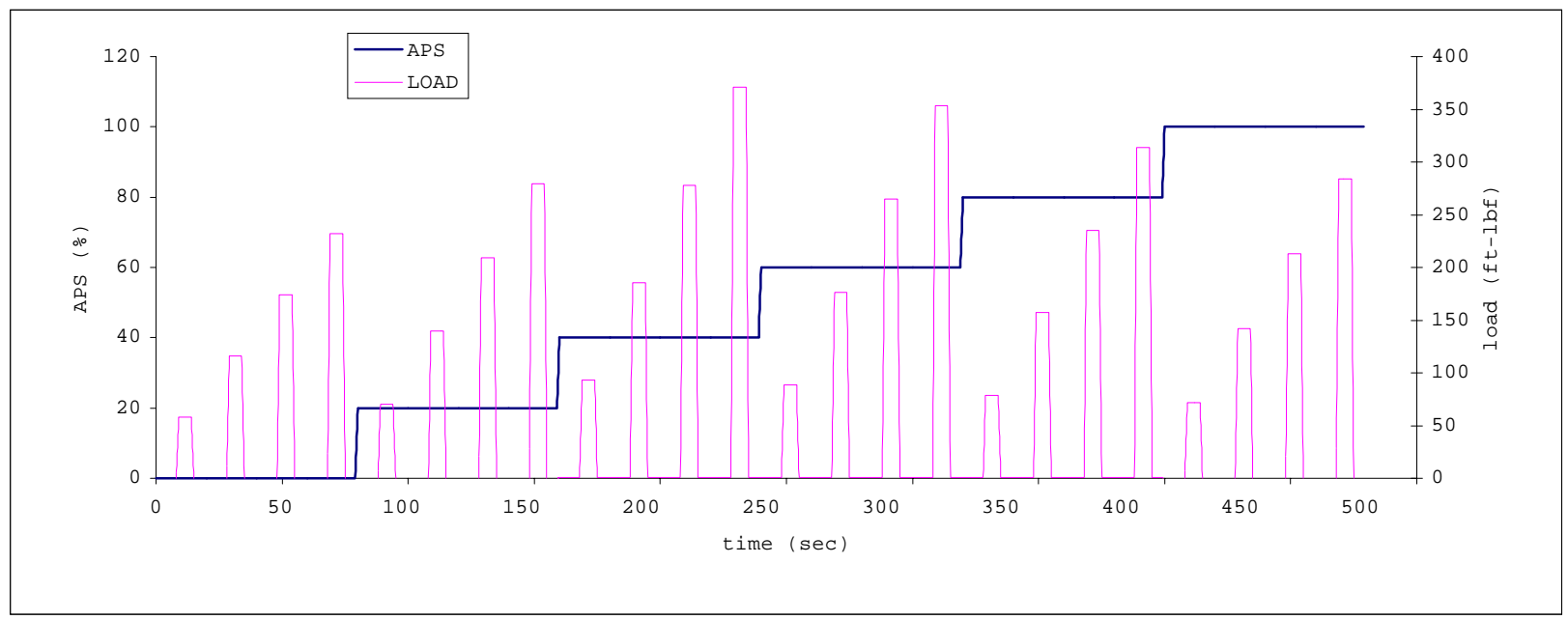

Figure 4.36a Pulsed load cycle at different positions of accelerator

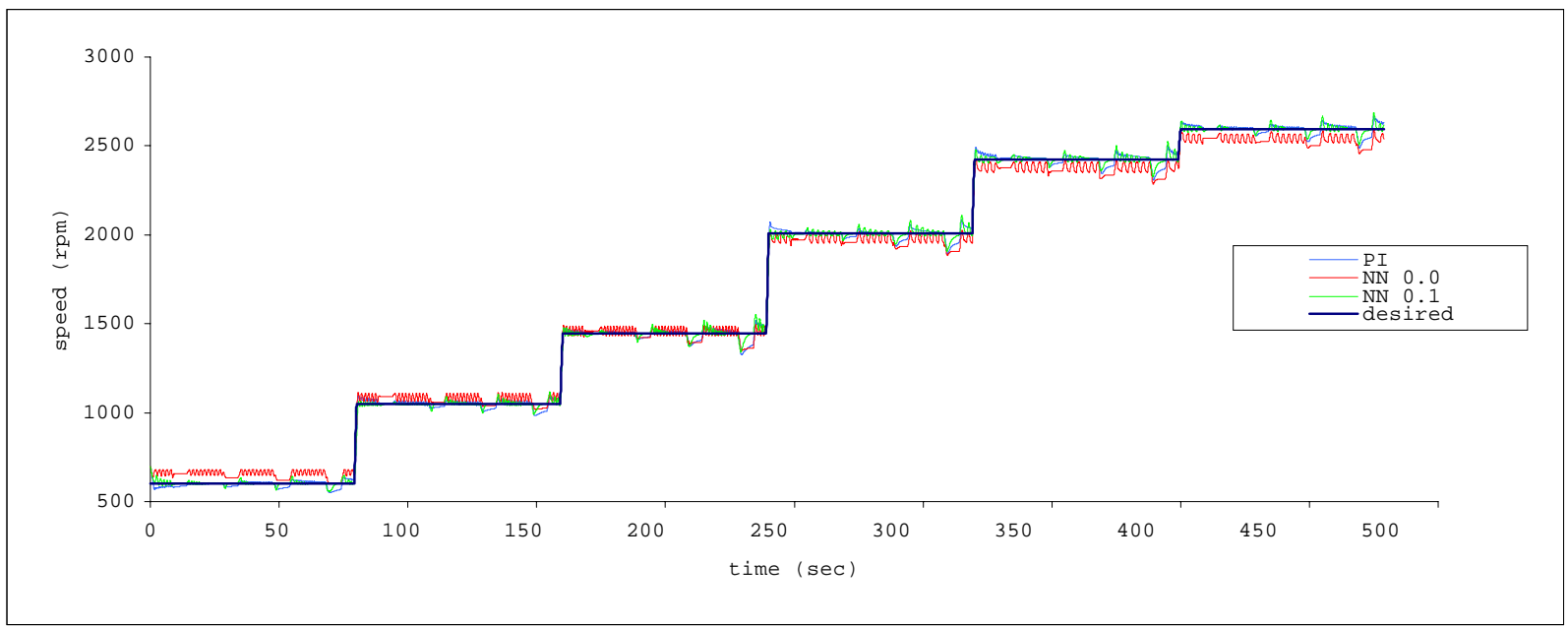

Figure 4.36b Speed tracking performance over pulsed load cycle at different positions of accelerator

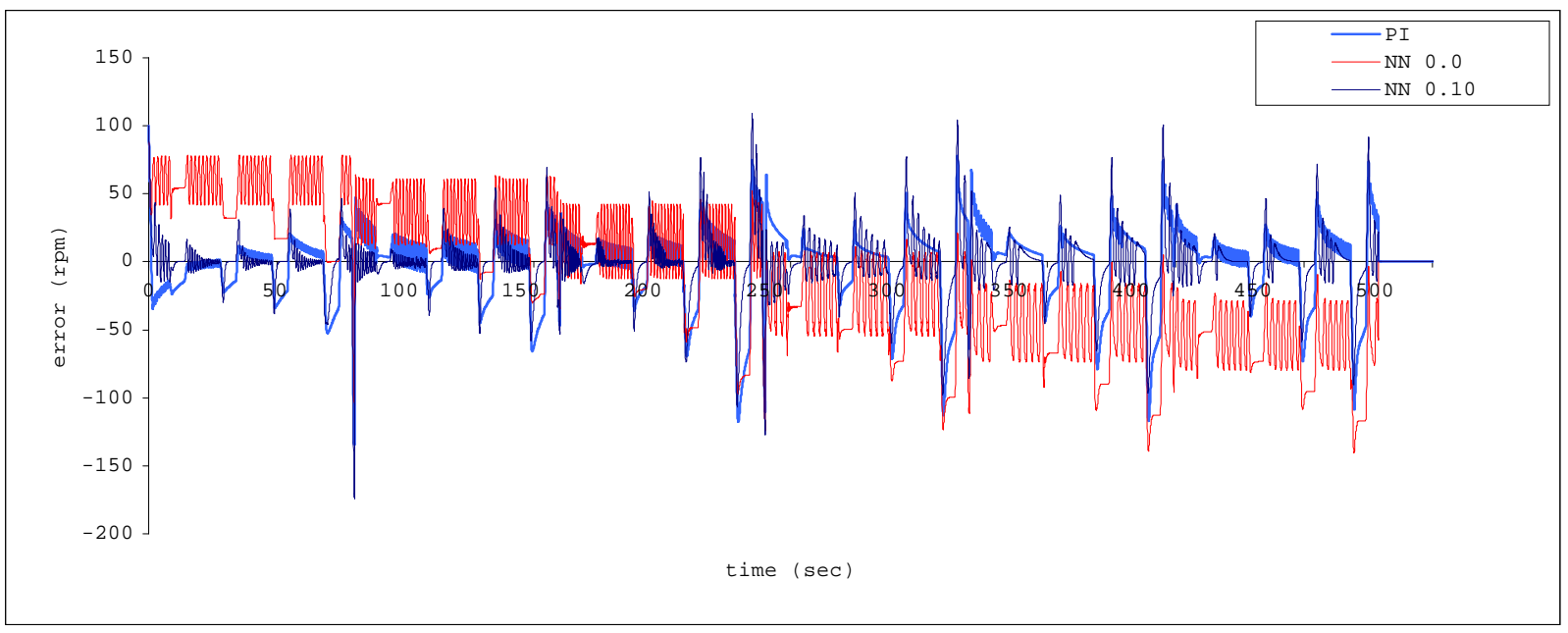

Figure 4.36c Error in speed tracking over pulsed load cycle at different positions of accelerator 


\subsection{Engine Torque Control with Embedded Speed Control}

The developed NN speed controller was embedded into the torque controller, which was previously developed for governed engine operation, as shown in Figure 4.37. The combined neural network layout is shown in Figure 4.38. The integral network consists of sixteen non-fully connected layers and is externally connected to the manifold dynamics NN and emissions predictions NNs. The speed controller weights were frozen at their trained values and the standard back-propagation algorithm was used to adapt the weights of the torque controller NN.

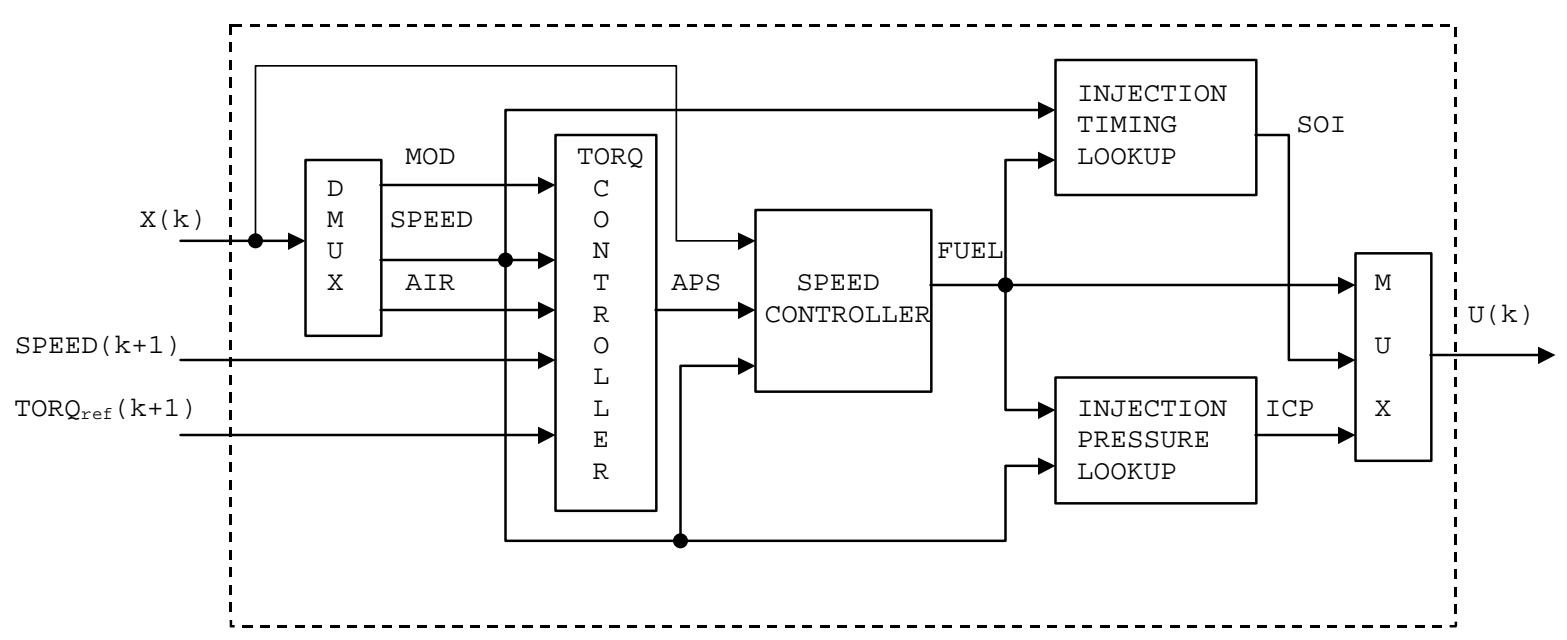

Figure 4.37 Schematic of the engine torque controller with embedded speed control

In order to guarantee initial stability, the NN torque controller was trained offline, in a batch mode, to mimic the behavior of a PI controller and the predicted torque value at the next time step was used as the current step desired value. The simulated test cycle consisted of a stepped torque cycle ( $0 \%$ to $100 \%$ at $5 \%$ increments of the maximum torque) at a constant engine speed, which spanned the range from $700 \mathrm{rpm}$ to $2220 \mathrm{rpm}$ with a step size of $380 \mathrm{rpm}$, as shown in Figure 4.39a. Excellent torque tracking was achieved using the PI control, as shown in Figure 4.39b, which provided an excellent initial start for the $\mathrm{NN}$ control, which was later adapted 
online. The steep rise in torque with the APS signal is clearly reflected on the very narrow band of the APS control command, shown in Figure 4.39b.

The closed-loop simulation was conducted for the FTP test cycle for the PI controller as well as the NN controller, which was adapted at a learning rate of $\lambda=0.001$. The torque tracking performance over the FTP for the PI and NN controllers is shown in Figure 4.40. The NN-based controller demonstrated better tracking performance than the PI controller. This result was further quantified through an analysis of the error in the positive torque tracking, shown in Table 4.11a, which includes the results for proportional governor type (II) for comparison purposes. The increased difficulty of the torque control with the tighter speed control is clearly demonstrated, especially for the PI controller. The integral results for the two controllers are shown in Table $4.11 \mathrm{~b}$

Table 4.11a Tracking analysis of positive torque over FTP with embedded speed control

\begin{tabular}{|c|c|c|c|c|}
\hline \multirow[t]{2}{*}{ measure } & \multicolumn{2}{|c|}{ governor NN } & \multicolumn{2}{|c|}{ governor II } \\
\hline & PI & NN & PI & NN \\
\hline e1 (ft-lbf) & 57.22 & 21.08 & 51.76 & 10.98 \\
\hline e2 (응 & 4.98 & 1.83 & 4.50 & 0.95 \\
\hline $\begin{array}{ll}\text { e3 } & (\%) \\
\end{array}$ & 11.81 & 1.19 & 12.04 & 0.44 \\
\hline c $(f t-l b f)$ & -6.33 & -1.42 & -6.46 & -0.66 \\
\hline$m(n / a)$ & 0.93767 & 1.00066 & 0.93659 & 1.00146 \\
\hline r2 (n/a) & 0.84334 & 0.97667 & 0.87033 & 0.99356 \\
\hline
\end{tabular}

Table 4.11b Integral results over FTP with embedded speed control

\begin{tabular}{|c|c|c|c|c|}
\hline \multirow[t]{2}{*}{ parameter } & \multicolumn{2}{|c|}{ governor NN } & \multicolumn{2}{|c|}{ governor II } \\
\hline & $\mathrm{PI}$ & NN & $\mathrm{PI}$ & NN \\
\hline ENERGY (bhp-h) & 8.981 & 9.967 & 8.985 & 10.016 \\
\hline ENERGY+ (bhp-h) & 10.923 & 11.445 & 10.674 & 11.397 \\
\hline FC $(g / b h p-h)$ & 182.148 & 185.211 & 188.791 & 186.783 \\
\hline THC $(g / b h p-h)$ & 0.200 & 0.196 & 0.204 & 0.194 \\
\hline $\mathrm{LCO}(\mathrm{g} / \mathrm{bhp}-\mathrm{h})$ & 1.310 & 1.548 & 1.792 & 1.927 \\
\hline $\mathrm{CO}_{2} \quad(\mathrm{~g} / \mathrm{bhp}-\mathrm{h})$ & 643.260 & 664.071 & 640.997 & 643.956 \\
\hline NOx $(g / b h p-h)$ & 5.384 & 5.530 & 5.451 & 5.465 \\
\hline PM $(g / b h p-h)$ & 0.077 & 0.086 & 0.090 & 0.091 \\
\hline
\end{tabular}




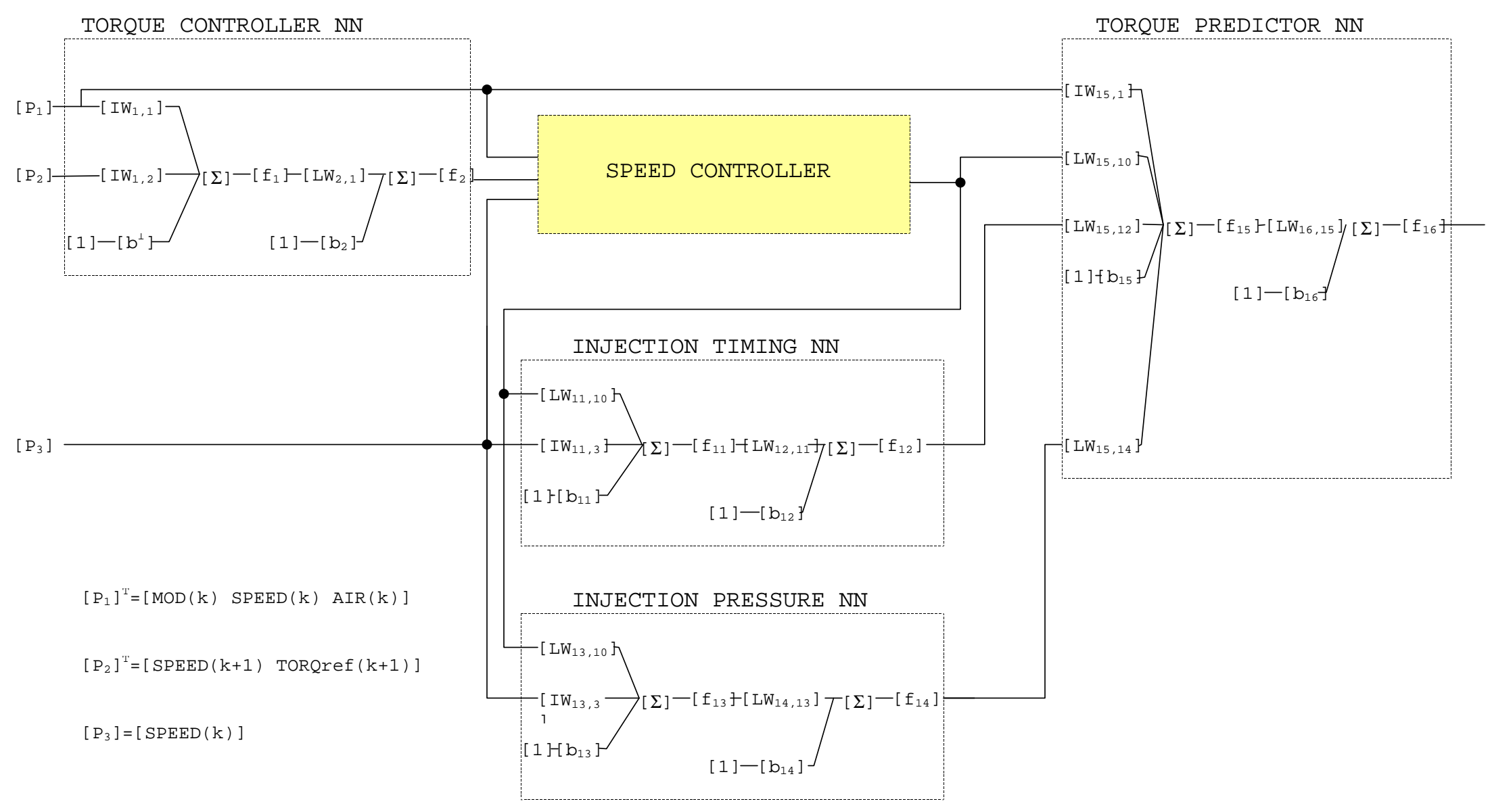

Figure 4.38 Combined neural network layout for torque controller with embedded speed control 


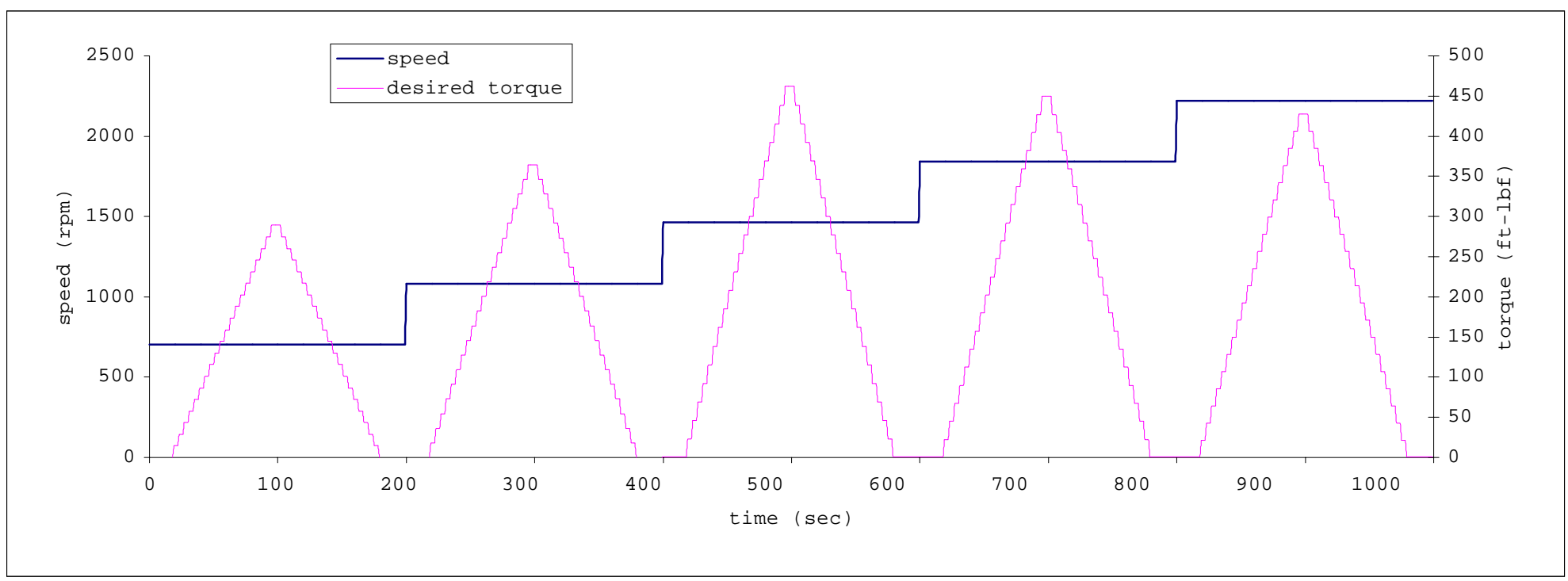

Figure 4.39a Stepped torque cycle at different engine speeds

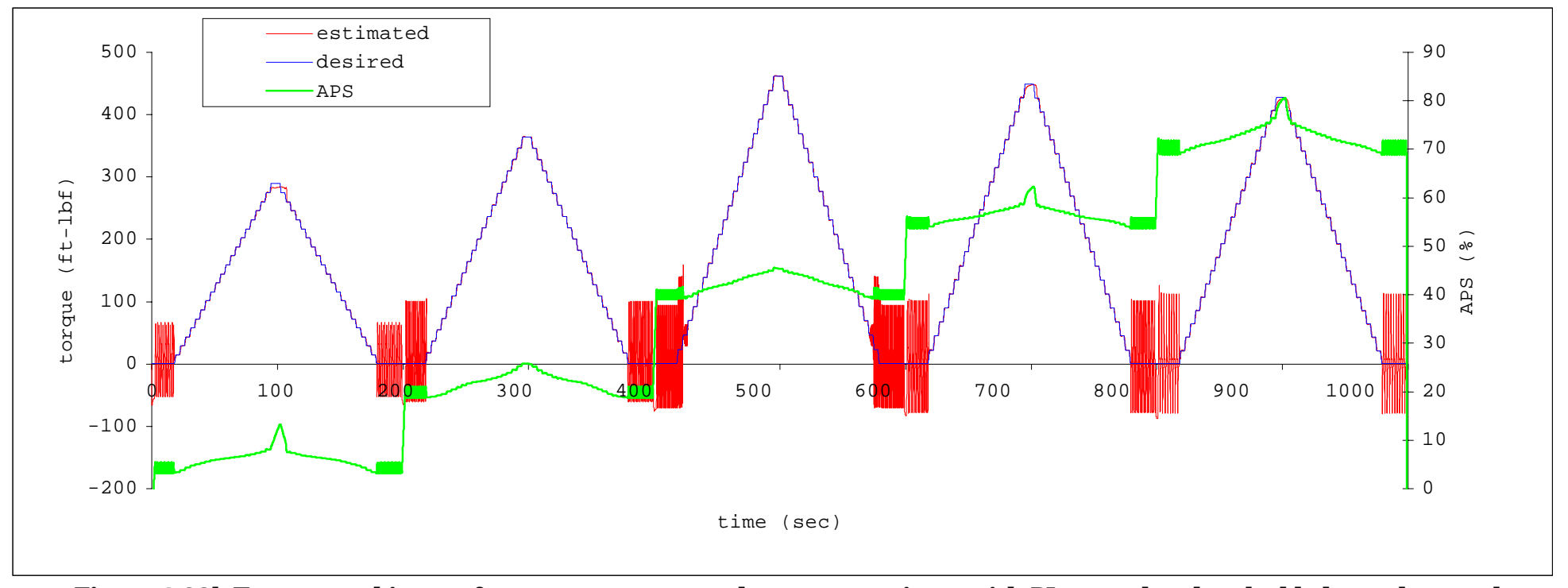

Figure 4.39b Torque tracking performance over stepped torque transients with PI control and embedded speed control 


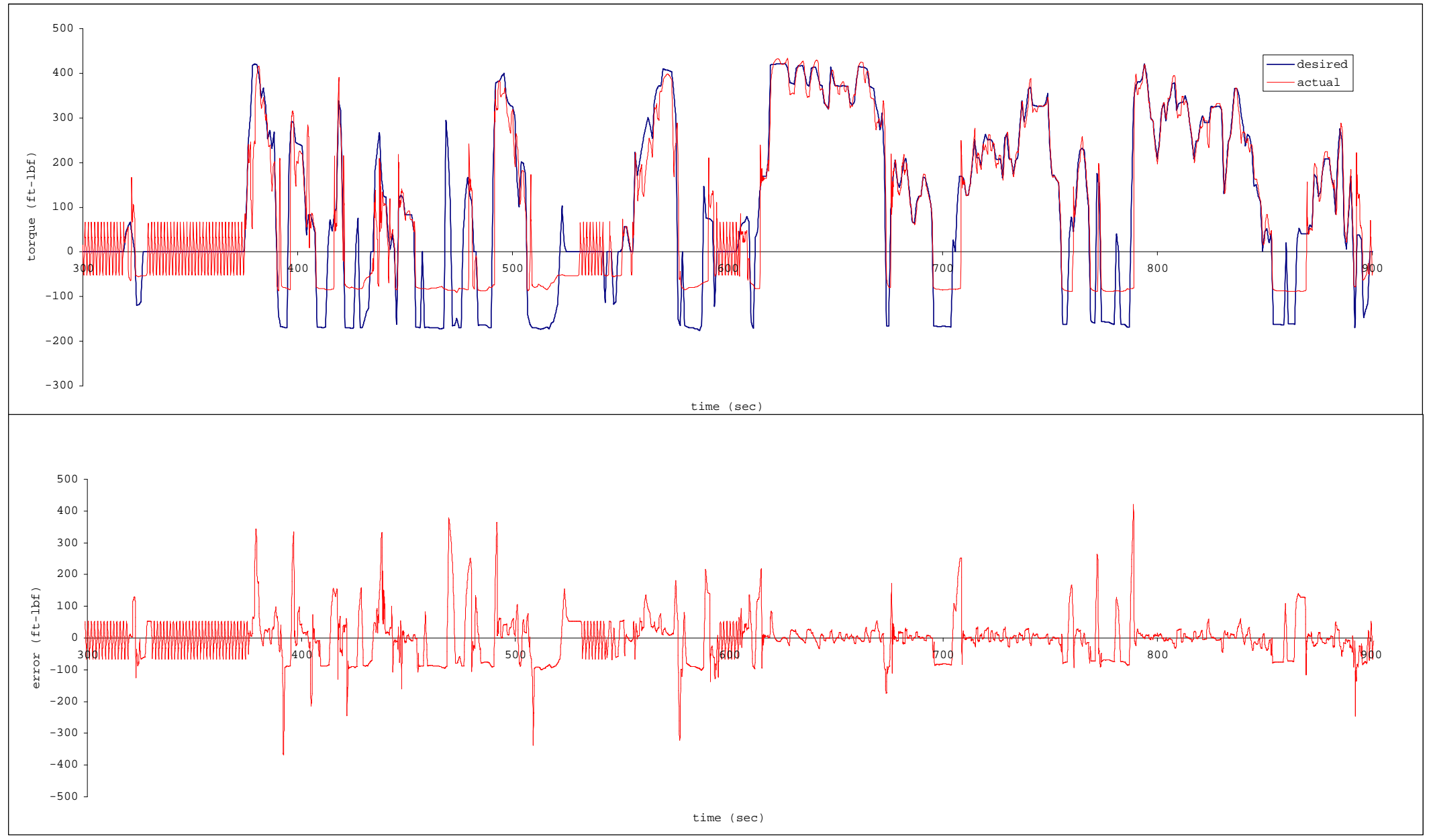

Figure 4.40a Desired and actual torque over the FTP cycle with PI torque control and embedded NN speed control 


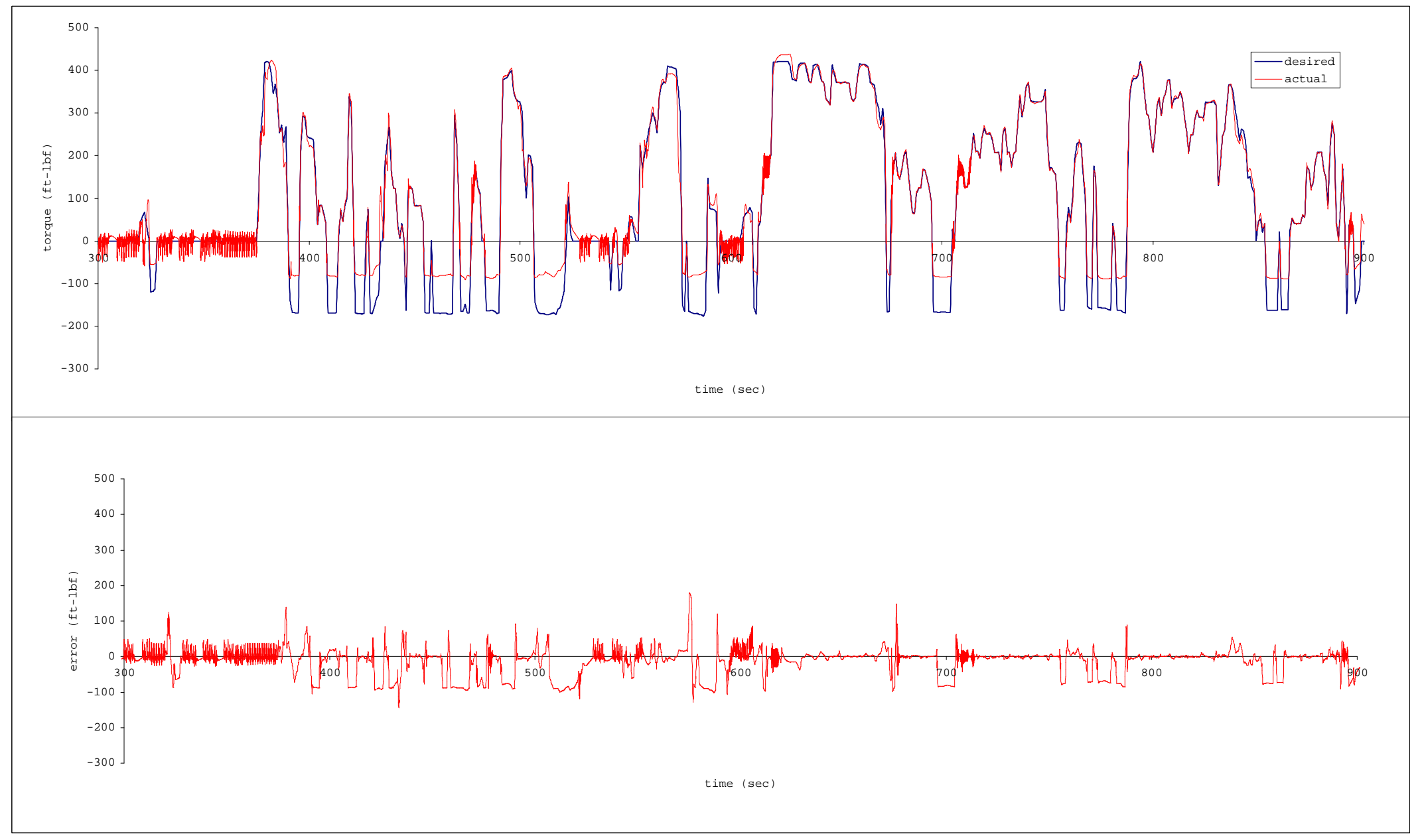

Figure 4.40b Desired and actual torque over the FTP cycle with NN, Ir=0.001, torque control and embedded NN speed control 


\section{CHAPTER 5}

\section{Summary and Conclusions}

\subsection{Summary}

The current investigation was aimed at the development and validation of a neural networkbased diesel engine mean value model and its application to engine control. These objectives have been fulfilled as follows:

Firstly, a neural network-based mean value model of a modern turbocharged direct injection diesel engine has been developed and validated. For a pre-specified engine speed schedule and control vector trajectory (open-loop simulation), the engine model was shown to produce accurate predictions of the turbocharger manifold charging dynamics and combustion efficiency through engine brake torque predictions. The mean value model was coupled with several individual neural network models that were used to predict exhaust gaseous and particulate emissions and satisfactory predictions were reported over several highly transient test schedules.

Secondly, a neural network-based engine torque controller has been developed and validated through simulation for both non-governed as well as governed engine operation. In order to guarantee the initial stability of the closed-loop performance, the inverse dynamics neural network was trained offline to emulate a classical proportional plus integral (PI) control. The original engine calibration maps for injection timing and injection pressure were modeled using neural networks and integrated with the inverse dynamics $\mathrm{NN}$ to form the torque controller, for the case of non-governed engine operation.

Two types of proportional governors have been considered where one governor employed a more aggressive fueling strategy than the other. The engine performance and exhaust gas emissions for both governors were quantified through simulation. The expected results of a 
steeper rise in torque and large excursions in transient emissions were predicted for the more aggressive fueling strategy. The governed fueling flow rate was modeled, using neural networks, and augmented to the torque controller layout, to simulate the case of governed engine operation.

The torque controller was integrated with the previously developed mean value engine model and adapted online using the standard back-propagation algorithm. For a pre-specified engine speed schedule and desired engine torque trajectory (on the closed-loop simulation), excellent torque tracking was predicted using the $\mathrm{NN}$ controller over transient operation compared to a classical PI controller, which was tuned heuristically. The variations in the integral results for brake specific emissions over the FTP cycle were examined, through simulation, for the maximum allowable deviation in the desired torque schedule within, the EPA test validation criteria.

Thirdly, a neural network-based all-speed governor was developed and validated through simulation. The speed controller featured a drivability measure where the accelerator position signal was used to look up a desired engine speed, typical of a drive-by-wire arrangement. A fueling limiting strategy was employed to shape the maximum engine torque characteristics. For a pre-specified engine load schedule and accelerator position trajectory, accurate tracking was predicted for the desired engine speed for both classical and NN-based controllers under high load transients.

Finally, the speed controller developed here was embedded in the engine torque control loop. The speed controller weights were frozen at their trained values and a standard back-propagation technique was used to adapt the weights of the torque controller online. Closed-loop performance was simulated over the FTP cycle and the neural network-based controller demonstrated excellent torque tracking compared to a classical PI control. 
A summary of the research efforts conducted as a part of this investigation includes:

(1) A review of the literature on mean value modeling of IC engines, neural-networks applications in internal combustion engines for system identification and control, and transient control of HD CI engines on test beds was conducted.

(2) A Navistar T444E was instrumented and tested on a transient DC dynamometer over several test cycles.

(3) An acquisition program was developed in Quick Basic, which allows for flexible setting of the sampling rate as well as channel assignments besides several other capabilities which were not directly related to the current investigation.

(4) A data reduction program was developed in the C programming language and was used to process the acquired data files and perform a multitude of tasks in a batch mode. These included data filtering and smoothing, generating integral results over any test cycle, calculating time shifts to maximize cross correlation of the nth derivative of any two variables, generating lookup maps of any $(\mathrm{m})$ variables in (n) independent variables, and variable estimation using previously generated maps.

(5) The mathematical formulation of the mean value model has been conducted which resulted in the appropriate identification of the functional dependencies required to construct appropriate input vectors to the neural network engine sub-models.

(6) Several MATLAB codes have been developed and used to preprocess the data, train and simulate the different neural networks, post process the output, and finally conduct open and closed-loop simulation of the integrated neural network models. Extensive use has been made of the MATLAB neural network toolbox. 


\subsection{Conclusions}

The major conclusions that can be drawn from the present investigation are:

(1) Neural network-based mean value diesel engine models have been shown to provide adequate characterization of the engine dynamic behavior as well as accurate predictions of engine brake torque and exhaust gas emissions of oxides of nitrogen and carbon dioxide. The approach is capable of adequately predicting continuous emissions of particulate matter, as measured by a tapered element oscillating microbalance (TEOM). The onset of large excursions or 'spikes' in the transient emissions of carbon monoxide and total hydrocarbons are accurately predicted although low level emissions of these exhaust constituents are difficult to estimate.

(2) The charging of the intake manifold of a turbocharged intercooled diesel engine has been found to have a generic second order dependence on the engine state vector and a first order dependence on the control vector. This result is supported through theoretical derivation and experimental validation. Also, the black box neural network-based approach of modeling the engine out-emissions as static non-linearity has been shown to be valid and to result in satisfactory predictions of the pollutants considered.

(3) Using neural networks to model the non-linear dependencies of the look up control parameters (e.g. injection timing and injection pressure) has been shown to be a very efficient alternative to stacked two-dimensional maps with successive corrections. The multivariable capability inherent in the neural network representation accommodates any extra dependency with ease, especially in view of the ever-expanding functionality of engine management electronics. 
(4) Using an estimate of the air mass flow rate, which is calculated from measured manifold gas pressure and temperature, as a state variable has been shown to augment the modeling of the naturally coupled intercooler and the turbocharger subsystems. This is considered advantageous compared to using manifold pressure as a state variable, where derived dependencies need to be later corrected for temperature variations.

(5) The a priori knowledge acquired by the developed neural network-based controllers through offline batch mode training was shown to be very useful in guaranteeing the initial stability of the closed-loop performance and avoiding local minimum problems when the standard back-propagation algorithm is used for online adaptation. The controller network weights are initialized to perform as a classical controller with predetermined stable closed-loop performance.

(6) Neural network torque controllers, with their adaptive ability, have demonstrated superior torque tracking compared to conventional PI controllers, which are heuristically tuned, especially for governed engine operation. The difficulty in achieving tight tracking of a prescribed torque schedule is mainly attributed to the highly non-linear dependence of fueling on the commanded accelerator position.

(7) Tighter tracking of engine torque was shown to result in higher brake specific emissions of carbon dioxide, oxides of nitrogen, and particulate matter, independent of the test schedule. It must be noted that this result, obtained through simulation, is strongly dependent on the engine calibration and may vary for other test engines.

(8) The EPA validation criteria for the prescribed engine torque over the FTP cycle was shown to allow for significant variations in brake specific emissions, especially 
particulate matter, total hydrocarbons, and carbon monoxide emissions, while still meeting the legal requirements for a valid engine certification test.

(9) For the purposes of offline synthesis of control algorithms, the coupling of the engine model with an emissions prediction model was shown to allow for the evaluation of the impact of the adopted control on transient emissions. For example, this technique can predict the onset of large excursions in particulate matter emissions at sharp transients for the case of a governor which implements an aggressive fueling strategy.

(10) Both classical and neural network-based speed controllers were shown to provide accurate tracking of the desired engine speed for a prescribed load schedule. Tighter speed tracking requires a more aggressive fueling strategy, which further complicates the task of torque tracking on the dynamometer, and results in uniformly higher exhaust emissions. 


\section{CHAPTER 6}

\section{Recommendations}

Based on the current research effort, the following is recommended:

(1) The distortion in the transient exhaust emissions measurements introduced by the sampling system (dilution tunnel, sampling lines, and analyzers) needs to be considered if accurate predictions of transient emissions are of primary concern. This effect is magnified for the case of implementing transient test cycles to generate the data required for constructing an emissions model. Though the maximum cross correlation scheme is capable of accurately accounting for the sampling system transport delays and analyzer response time, it does not consider the distortion in the measured signal due to axial dispersion. It is proposed either to de-convolute the measured signal through identification of the sampling system or to implement fast response analyzers capable of quantifying raw emissions on a cycle by cycle basis. It must be noted that although fast response analyzers exist for hydrocarbons and nitric oxide, no equivalent instruments currently exist for carbon monoxide and particulate matter.

(2) Transient arbitrary cycles generated on a semi-random basis are strongly recommended for generating the data required to construct a mean value model. The advantage of this approach is twofold. Firstly, transient operation is naturally included especially for the case of identifying manifold charging dynamics. Secondly, the time expended on actual testing on the dynamometer is substantially reduced compared to elaborate sweeps of the evergrowing number of control parameters at steady state operation. Although the delays and distortion in the emissions signal introduced by the sampling train, is eliminated for steady 
state operation, transient testing is still an attractive approach especially if recommendation (1) is implemented.

(3) For the purpose of online model-based control implementation of the mean value approach developed here, a reduced order model is proposed. In this approach, the lookup control variables are eliminated from the control vector. For example, the control vector is reduced to fuel injection quantity if injection timing and injection pressure are looked up from predetermined calibration maps. This is motivated by the fact that their static functional dependencies are already acknowledged in the input state and control vectors.

(4) The speed governor developed in the current investigation does not implement a smoke limiting strategy, which is necessary for practical implementation. Two approaches are proposed. In the first approach a smoke-limiting look up is introduced similar to the one used for the maximum torque-limiting map. Under transient operation, this map would essentially act as an air to fuel ratio limiter with a dependency on engine speed and scavenged air proposed. The second approach makes use of the particulate matter (PM) predictor developed here to act as a virtual sensor for dynamic control of transient PM emissions.

(5) The concept of online control of oxides of nitrogen (NOx) emissions is very attractive since NOx is of primary concern in modern direct injection diesel engines employing high injection pressure and electronic fuel control. This is further supported by the present lack of catalytic aftertreatment of NOx emissions suitable for mobile applications. A control strategy that allows NOx emissions up to a constrained limit regardless of the operating mode is beneficial, particularly in view of the direction of current emissions legislation. The mean value model developed here with its capability to accurately predict NOx 
emissions lends itself as a very useful tool in the synthesis of such a strategy. Two difficulties must be acknowledged. Firstly, it is difficult to define a target value for the proposed closed loop strategy, which is realizable under all operating conditions. Secondly, the proposed control parameter (such as injection timing) needs to be exercised extensively on the test stand and its causal effect needs to be accurately identified while the problems raised in (1) complicates this task.

(6) For the task of speed and torque control considered here, the standard back-propagation performed adequately and is recommended for online adaptation in future implementation because of the simplicity of the underlying algorithm. An aspect of interest is how often one needs to adapt the controller and how beneficial it is to revert to batch training over a sliding window compared to incremental adaptation every time step.

(7) The current investigation did not provide any theoretical proof of stability for the neural network controllers developed here. Stability needs to be considered if certification becomes of concern. One might opt to use radial basis functions network, with proven stability, in place of the single hidden layer perceptron networks exclusively used in the current investigation. 


\section{REFERENCES}

Atkinson C., Long T., and Hanzevack E., 'Virtual Sensing: a Neural Network-based Intelligent Performance and Emissions Prediction System for On-Board Diagnostics and Engine Control', SAE Paper 980516, 1998a.

Atkinson C., Clark N., Thompson G., Gilbert M., and Yacoub Y., 'Navistar T444E Diesel Engine Test Results', Internal Report, MAE Department, West Virginia University', July 1998b.

Asik J., Peters J., Meyer G., and Tang D., 'Transient A/F Estimation and Control Using a Neural Network', SAE Paper 970619, 1997.

Aufinger H., and Cichocki R., 'Challenges to Meet Heavy Duty Diesel Engine Euro II and US 1998 Emission Regulations', SAE Paper 931637, 1993.

Ayeb M., Lichtenthaeler D., Winsel T., and Theuerkauf H., 'SI Modeling Using Neural Networks', SAE Paper 980790, 1998.

Brown D. and Thompson S., 'A Novel Approach to Engine Torque Speed Control', SAE Paper 831302, 1983.

Clark C., May M., and Challen B., 'Transient Testing of Diesel Engines', SAE Paper 840348, 1984.

Code of Federal Regulations, Title 40, Part 86, Subpart N, 'Emission Regulations for New Otto Cycle and Diesel Heavy Duty Engines; Gaseous and Particulate Exhaust Test Procedures', Environmental Protection Agency, 1996.

De Nicolao G., Scattolini R., and Siviero C., 'Modeling the Volumetric Efficiency of IC Engines: Parametric, Non-Parametric and Neural Techniques', Control Eng. Practice, Vol. 4, No. 10, pp. 1405-1415, 1996.

Gissinger G., Renard R., and Hassenforder M., 'Model Based Design and Control of Diesel Engines', SAE Paper 890568, 1989.

Hagan M. and Menhaj M., 'Training feed-forward networks with the Marquardt algorithm', IEEE Transactions on Neural Networks, vol. 5, no. 6, pp. 989-993, 1994.

Hagan M., Demuth H., and Beale M., 'Neural Network Design', PWS Publishing Company, Boston, MA, 1996.

Hales J., and May M., 'Transient Cycle Emissions Reduction at Ricardo - 1988 and Beyond', SAE Paper 860456, 1986. 
Hanzevack E., Long T., Atkinson C., and Traver M., 'Virtual Sensors for Spark Ignition Engines Using Neural Networks', Proceedings of the American Controls Conference, Albuquerque, NM, June 1997.

Haykin S., 'Neural Networks: A Comprehensive Foundation', Macmillan College Publishing Company, Inc., Englewood Cliffs, NJ, 1994.

Hendricks E., 'A Compact, Comprehensive Model of Large Turbocharged, Two-Stroke Diesel Engines', SAE Paper 861190, 1986.

Hendricks E. and Poulsen N., 'Minimum Energy Control of a Large Diesel Engine', SAE Paper 861191, 1986.

Hendricks E., 'Mean Value Modeling of Large Turbocharged Two-Stroke Diesel Engines', SAE Paper 890564, 1989.

Hendricks E. and Sorenson S., 'Mean Value Modeling of Spark Ignition Engines', SAE Paper 900616, 1990.

Heywood J., 'Internal Combustion Engine Fundamentals', McGraw-Hill, Inc., NY, 1988.

Hornick K., Stinchcombe M., and White H., 'Multilayer feed forward networks are universal approximators', Neural Networks, vol. 2, no. 5, pp. 359-366, 1989.

Hower M., Mueller R., Oehlerking D., and Zielke M., 'The New Navistar T 444E Direct Injection Turbocharged Diesel Engine', SAE Paper 930269, 1993.

Isermann R., Stefan S., and Schaffnit J., 'Modeling and Real-Time Simulation of Diesel Engines for Control Design', SAE Paper 980796, 1998.

Jensen J., Kristensen A., Sorenson S., Houbak N., and Hendricks E., 'Mean Value Modeling of a Small Turbocharged Diesel Engine', SAE Paper 910070, 1991.

Kao M. and Moskwa J., 'Turbocharged Diesel Engine Modeling for Nonlinear Engine Control and State Estimation', Transactions of the ASME Journal of Dynamic Systems, Measurement, and Control, vol. 117, pp. 20-30, March 1995a.

Kao M. and Moskwa J., 'Nonlinear Diesel Engine Control and Cylinder Pressure Observation', Transactions of the ASME Journal of Dynamic Systems, Measurement, and Control, vol. 117, pp. 183-192, June 1995b.

Kawakami M., Tanabe H., Matsuda S., Sato K., 'Environmental Control of Advanced MediumSpeed Engines', Paper No: 94-ICE-14, Presented at the Energy Sources Technology Conference and Exhibition, New Orleans, Louisiana - Jan 23-27, 1994. 
Koustas J. and Watson N., 'A Transient Diesel Test Bed with Direct Digital Control', SAE Paper 840347, 1984.

Lenz U. and Schroeder D., 'Artificial Intelligence for Combustion Engine Control', SAE Paper 960328, 1996.

Lenz U. and Schroeder D., 'Transient Air-Fuel Ratio Control Using Artificial Intelligence', SAE Paper 970618, 1997.

Lenz U. and Schroeder D., 'Air-Fuel Ratio Control for Direct Injecting Combustion Engines using Neural Networks', SAE Paper 981060, 1998.

Leonhardt S., Ludwig C., and Shwarz R., 'Real Time Supervision For Diesel Engine Injection', Control Engineering Practice, Vol. 3, No. 7, pp. 1003-1010, April 1995.

Lippmann R., 'An Introduction to Computing with Neural Nets', IEEE ASSP Magazine, April, 1987.

Ludwig C. and Ayoubi M., 'Fault Detection Schemes for a Diesel Engine Turbocharger', Proceedings of the American Control Conference, Seattle, WA, pp. 2118-2122, June 1995.

Lukich M., Barnes T., and Maddock J., 'Rapid Prototyping of Control Strategies for Embedded Systems', SAE Paper 951197, 1995.

Mencik Z. and Blumberg P., 'Representation of Engine Data by Multivariate Least-Squares Regression', SAE Paper 780288, 1978.

Miller F., 'Transient Engine Testing Torque and Speed Compliance', MSME. Thesis, Department of Mechanical and Aerospace Engineering, West Virginia University, 1995.

Mori K., 'Worldwide Trends in Heavy-Duty Diesel Engine Exhaust Emission Legislation and Compliance Technologies’, SAE Paper 970753, 1997.

Müller R. and Hemberger H., 'Neural Adaptive Ignition Control', SAE Paper 981057, 1998.

Narendra K. and Parthasarathy K., 'Identification and Control of Dynamical Systems Using Neural Networks', IEEE Transactions on Neural Networks, vol. 1., no. 1, pp. 4-27, March 1990.

Nelson S., Filipi Z., and Assanis D., 'The Use of Neural Nets for Matching Compressors with Diesel Engines’, ASME-ICE Spring Technical Conference, Vol. 26-3, 1996.

Noble A., Beaumont A., and Mercer A., 'Predictive Control Applied to Transient Engine Testbeds', SAE Paper 880487, 1988. 
Odaka M., Tsukamoto Y., Yagi H., Hashizume T., 'Development of Automatic Engine Control System with Predictive Control and its Application for Heavy Duty Diesel Engines', SAE Paper 900234, 1990.

Onder C.and Geering H., 'Model-Based Engine Calibration for Best Fuel Efficiency', SAE Paper 950983, 1995.

Ortmann S., Rychetsky M., Glesner M., Groppo R., Tubetti P., and Morra G., 'Engine Knock Estimation using Neural Networks based on a real-world Database', SAE Paper 980513, 1998.

Pilley A., Noble A., Beumont A., Needham J., and Porter B., 'Optimization of Heavy-Duty Diesel Engine Transient Emissions by Advanced Control of a Variable Geometry Turbocharger', SAE Paper 890395, 1989.

Ramli M. and Morris A., 'Application of Neural Networks to the Automotive Engine Problem', Int. J. of Vehicle Design, vol. 14, nos. 2/3, pp. 184-193, 1993.

Salem F. and Gharbi A., 'Temporal Neuro-Control of Idle Engine Speed', Proceedings of the 1996 IEEE International Symposium on Intelligent Control, Dearborn MI, pp. 396-401, September 1996.

Scaife M., Charlton S., and Mobley C., ‘A Neural Network for Fault Recognition', SAE Paper 930861, 1993.

Schmitz G., Oligschläger U., Eifler G., and Lechner H., 'Automated System for Optimized Calibration of Engine Management Systems’, SAE Paper 940151, 1994.

Shayler P., Goodman M., and Ma T., 'Transient Air/Fuel Ratio Control of an SI Engine Using Neural Networks', SAE Paper 960326, 1996.

Simpson P., 'Artificial Neural Systems: Foundations, Paradigms, Applications, and Implementations', Pergamon Press, Inc., Elmsford, NY, 1990.

Stobart R., May A., Challen B., and Morel T., 'Modeling for Diesel Engine Control: The CPower Environment', SAE 980794, 1998.

Tsai S. and Goyal M., 'Dynamic Turbocharged Diesel Engine Model for Control analysis and Design', SAE Paper 860455, 1986.

Tuken T., Fullmer R., and VanGerpen J., 'Modelling, Identification, and Torque Control of a Diesel Engine for Transient Test Cycles', SAE Paper 900235, 1990.

Voigt K., 'Model Based On Line Optimization For Modern Engine Management Systems', SAE Paper 931668, 1993.

Voos W., 'Dynamic Engine Testing', SAE Paper 920254, 1992. 
Wachter W., 'Analysis of Transient Emission Data of a Model Year 1991 Heavy Duty Diesel Engine', SAE Paper 900443, 1990.

Watson N., 'Dynamic Turbocharged Diesel Engine Simulator for Electronic Control System Development', Transactions of the ASME Journal of Dynamic Systems, Measurement, and Control, vol. 106, pp. 27-45, March 1984.

Weeks R. and Moskwa J., 'Automotive Engine Modeling for Real-Time Control Using MATLAB/SIMULINK', SAE Paper 950417, 1995.

Wu Z. and Lee A., 'Misfire Detection Using a Dynamic Neural Network with Output Feedback', SAE Paper 980515, 1998.

Zhang N., Perumpral J., Byler R., and Shaffer S., 'Diesel Engine Control Based on an ARMA Model', Transactions of the American Society of Agricultural Engineers, Vol. 32(4), pp. 11121120, July-August 1989. 


\section{VITA}

\section{Yasser Yacoub}

\section{PERSONAL INFORMATION}

Name: $\quad$ Yasser Mohamed Sayed Yacoub

Birth date/place: $\quad$ Dec $9^{\text {th }} 1968$ in Giza Egypt

Nationality: Egyptian

Family Status: Married

EDUCATIONAL HISTORY

From To Institute

Certificate Obtained Grade/GPA

May 95-May 99 West Virginia University

Ph.D.

$4.0 / 4.0$

MAE

Mechanical Engineering

Aug 93-May $95 \quad$ West Virginia University

M.S.

$4.0 / 4.0$

MAE

Aerospace Engineering

Aug 86-May $91 \quad$ Cairo University

$\mathrm{AE}$

B.Sc. with honor

$90.05 / 100$

Aerospace Engineering

\section{PUBLICATIONS}

Loth J., Yacoub Y., Loth E., " A Novel Staged Internal Combustion Engine", AIAA/SAE/ASME/ASEE 30th Joint Propulsion Conference and Exhibit, June, 1994 Indianapolis, IN.

Bata R., Yacoub Y., Wang W., Gambino M., Rideout G., "Heavy Duty Testing Cycles: Survey \& Comparison", Transactions of the Society of Automotive Engineers, SAE Paper No 942263, 1994.

Yacoub Y., Bata R., Lyons D., “ A Simulation Model of a Chassis Dynamometer: Model Development \& Cycles Application ", International Journal of Computer Applications in Technology, Vol. 9, Number 2/3, 1996.

Yacoub Y., Bata R., “ Model Development and Validation of a Spark-Ignition Single Cylinder Engine Performance and Emissions", ASME Journal of Engineering for Gas Turbines and Power, Vol. 120, Jan. 1998. 
Yacoub Y., Marbun L., Bata R., 'A Numerical Investigation of the Cooling Effect of Compressed Natural Gas Throttling on Engine Delivery Ratio', ASME Journal of Engineering for Gas Turbines and Power, Vol. 121, Jan 1999. Also, Presented at ASME - ICE Spring Technical Conference, Fort Collins, Colorado, April 1997.

Yacoub Y., Bata R., Gautam M., Martin D., 'The Performance Characteristics of C1-C5 Alcohol-Gasoline Blends With Matched Oxygen Content in A Single Cylinder Engine', Presented at American Chemical Society-Fuel Division, 213th ACS National Meeting, San Francisco, California, April 13-17, 1997

Yacoub Y., Bata R., Gautam M., 'The Performance And Emission Characteristics of C1-C5 Alcohol-Gasoline Blends With Matched Oxygen Content in A Single Cylinder Engine', Journal of Power and Energy, Vol. 212 Part A Proceedings of the Institution of Mechanical Engineers Part A., Suffolk, UK.

Yacoub Y., 'Method Procedures for Sampling Aldehyde and Ketone using 2,4dinitirophenylhydrazine -a review', Accepted for publication in the Journal of Automobile Engineering - Proceedings of the Institution of Mechanical Engineers Part D., Suffolk, UK.

Yacoub Y., Atkinson C., 'Modularity in SI Engines - A Review of Its Benefits, Implementation, and Limitations', SAE Paper No: 982688, Presented in the 1998 SAE International Fall Fuels \& Lubricants Meeting \& Exposition, October 19-22, 1998 in San Francisco, California.

Yacoub Y., Atkinson C., 'An Air Cycle Analysis of Alternatives to Throttling in the Otto Cycle Engine', To appear in the ICE-ASME 1999 Spring Engine Technology Conference, April 25-28, 1999 in Columbus, Indiana.

Yacoub Y., Atkinson C., 'Evaluation of Oxides of Nitrogen Emissions for the Purpose of Their Transient Regulation from a Direct Injection Diesel Engine', to appear in a future presentation. 